\title{
Calibration of the Accuscan II IN Vivo System for High Energy Lung Counting
}

\author{
O. R. Perry
}

July 2011

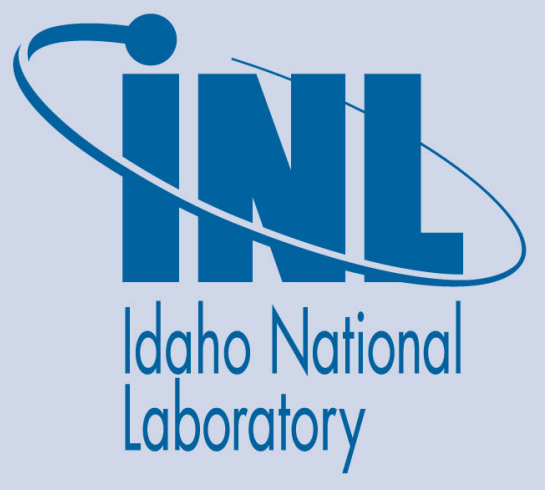

The INL is a U.S. Department of Energy National Laboratory operated by Battelle Energy Alliance 


\section{DISCLAIMER}

This information was prepared as an account of work sponsored by an agency of the U.S. Government. Neither the U.S. Government nor any agency thereof, nor any of their employees, makes any warranty, expressed or implied, or assumes any legal liability or responsibility for the accuracy, completeness, or usefulness, of any information, apparatus, product, or process disclosed, or represents that its use would not infringe privately owned rights. References herein to any specific commercial product, process, or service by trade name, trade mark, manufacturer, or otherwise, does not necessarily constitute or imply its endorsement, recommendation, or favoring by the U.S. Government or any agency thereof. The views and opinions of authors expressed herein do not necessarily state or reflect those of the U.S. Government or any agency thereof. 
INL/EXT-11-22670

Revision 0

\section{Calibration of the Accuscan II IN Vivo System for High Energy Lung Counting}

O. R. Perry

July 2011

Idaho National Laboratory

Health Physics Dosimetry Laboratory

Idaho Falls, Idaho 83415

http://www.inl.gov

Prepared for the

U.S. Department of Energy

Office of Nuclear Energy

Under DOE Idaho Operations Office

Contract DE-AC07-05ID14517 



\section{Health Physics Dosimetry Laboratory}

\section{Calibration of the Accuscan II IN Vivo System for High Energy Lung Counting}

INL/EXT-11-22670

Revision 0

July 2011

Approved by:
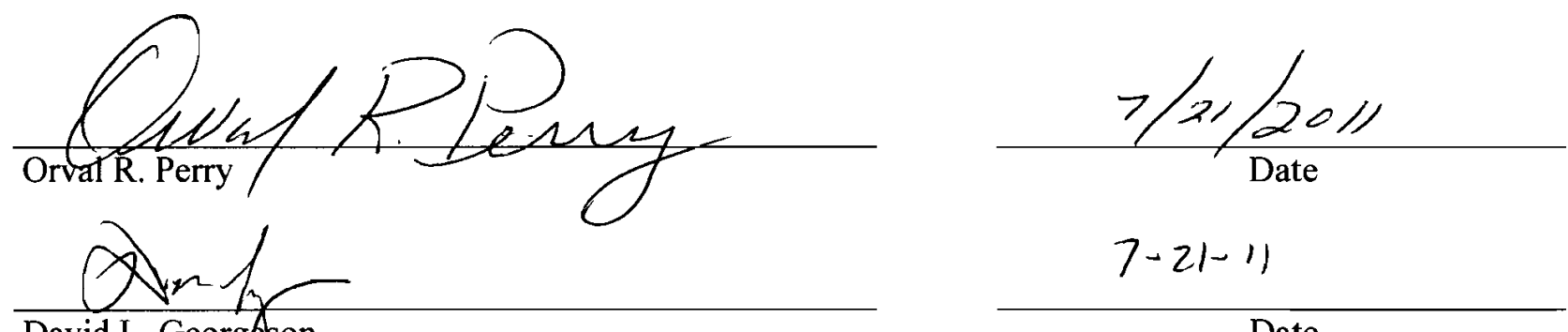

David L. Georgeson

Internal Dosimety Technical Lead
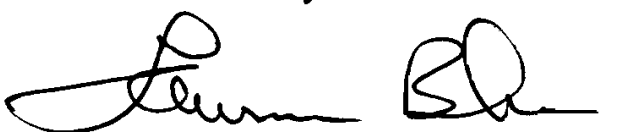

Lawrence L. Burke

Health Physics Dosimetry Laboratory Manger

Date

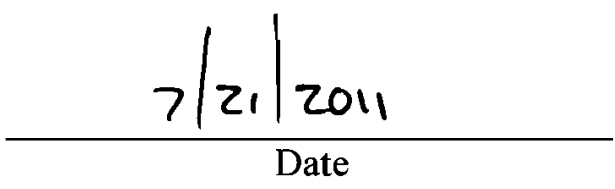





\begin{abstract}
This report describes the April 2011 calibration of the Accuscan II HpGe In Vivo system for high energy lung counting. The source used for the calibration was a NIST traceable lung set manufactured at the University of Cincinnati \#UCLL43AMEU \& UCSL43AMEU containing Am-241 and Eu-152 with energies from $26 \mathrm{keV}$ to $1408 \mathrm{keV}$. The lung set was used in conjunction with a Realistic Torso phantom. The phantom was placed on the RMC II counting table (with pins removed) between the v-ridges on the backwall of the Accuscan II counter. The top of the detector housing was positioned perpendicular to the junction of the phantom clavicle with the sternum. This position places the approximate center line of the detector housing with the center of the lungs. The energy and efficiency calibrations were performed using a Realistic Torso phantom (Appendix I) and the University of Cincinnati lung set. This report includes an overview introduction and records for the energy/FWHM and efficiency calibration including performance verification and validation counting. The Accuscan II system was successfully calibrated for high energy lung counting and verified in accordance with ANSI/HPS N13.30-1996 criteria.
\end{abstract}




\section{CONTENTS}

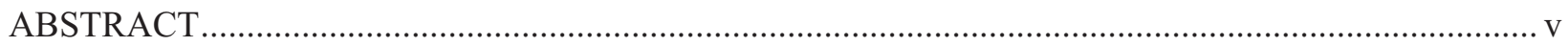

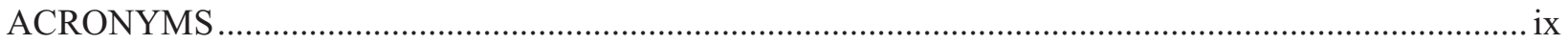

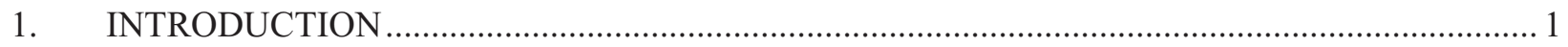

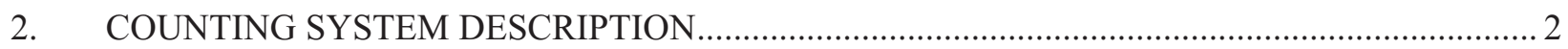

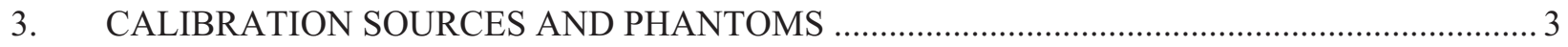

4. DETECTOR AND LYNX DIGITAL AMPLIFIER TESTING …............................................ 4

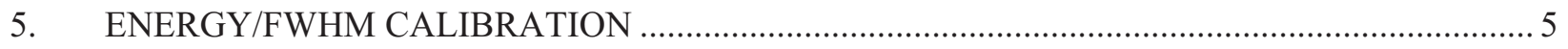

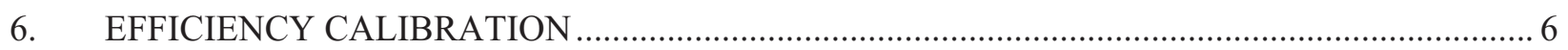

7. CALIBRATION COUNT REANALYSIS _....................................................................... 7

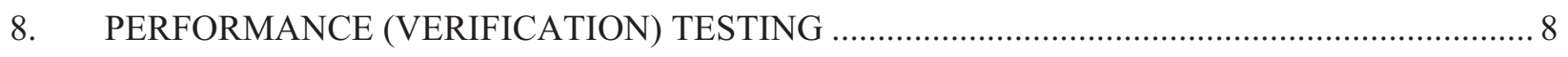

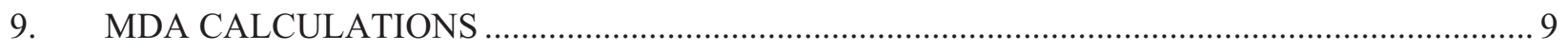

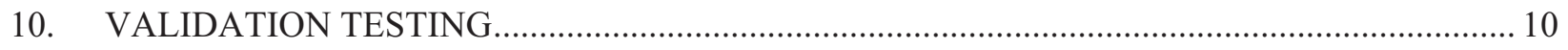

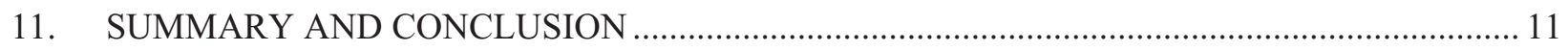

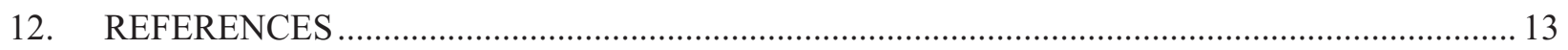

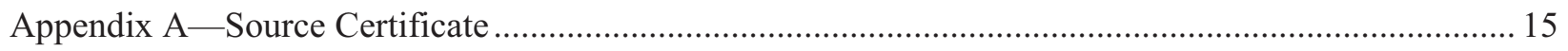

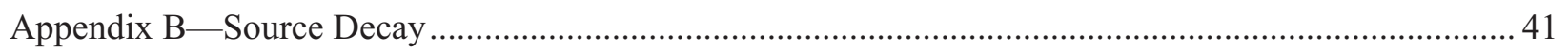

Appendix C_Energy/FWH and Low Tail Calibration Documentation................................................ 45

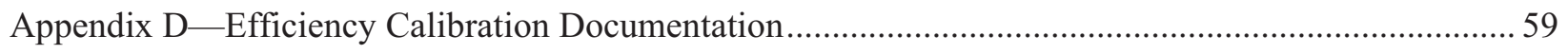

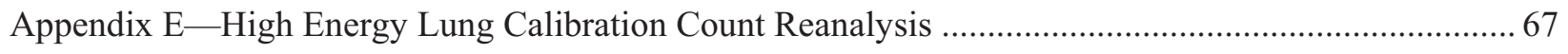

Appendix F-Accuscan II High Energy Lung Calibration Verification ................................................. 79

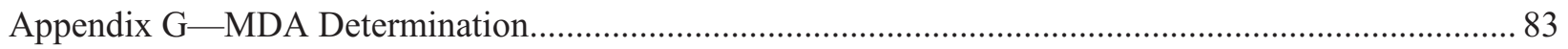

Appendix H-Calibration Validation Using a DOE Manufactured BOMAB \#MFP-1-001 .................... 103

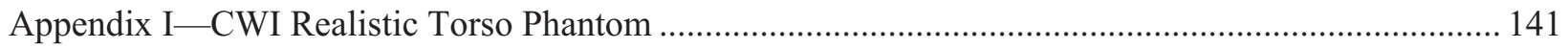

\section{TABLES}

Table 1. Calibration summary table. 


\section{ACRONYMS}

ANSI. American National Standard Institute

$A S F . \quad$ Analysis Sequence File

BE. Broad Energy

CFA Central Facility Area

CPS. Counts per second

FWHM. Full With at Half Maximum

HpGe. High Purity Germanium -also HPGe

HPL. Health Physics Laboratory

HPS. Health Physics Society

INL. Idaho National Laboratory

MDA. Minimum Detectable Activity

NIST. National Institute of Standards and Testing

PC. Personal Computer

RMC. Radiation Management Corporation

WBC Whole Body Counting 


\section{Calibration of the Accuscan II IN Vivo System for High Energy Lung Counting}

\section{INTRODUCTION}

This document describes the April 2011 calibration of the Accuscan II in vivo system for high energy lung counting. The new calibration files were stored on the system disk and made available for high energy (HE) lung counting after all verification counts were completed. This is the initial calibration of the Accuscan II for personnel HE lung counting.

The energy/FWHM-Low Tail calibration was performed on April 5, 2011 and is documented in Appendix C. A University of Cincinnati manufactured (NIST traceable) lung set containing Am-241 and Eu-152 was used together with a Realistic Torso phantom to perform the calibration. This source (UCLL43AMEU and UCSL43AMEU) is NIST traceable and is documented in Appendix A. The source has a reference date of June 24, 1996 with the current activity documented in Appendix B. The Realistic Torso phantom was used for both the energy and efficiency calibrations. The lung set was positioned in the Realistic Torso phantom and counted for 7228 seconds for the energy calibration and 7226 seconds for the efficiency calibration with the top of the detector housing position perpendicular to the junction of the phantom clavicle with the sternum.

An efficiency calibration was performed subsequent to the energy/FWHM calibration on April 5, 2011 and is documented in Appendix D. To calibrate this counting geometry, a mathematical function relating efficiency versus photopeak energy was created. The mathematical efficiency function generated on April 5, 2011 is expressed as a Dual fit using a $5^{\text {th }}$ order polynomial with no cross over. The efficiency is determined empirically by measuring known standards and using least squares fitting techniques. The efficiency function is stored as a calibration file, which is used to analyze spectra, collected under the same counting conditions.

Replicate performance (verification) counts (using the new calibration files) were performed to confirm that the accuracy and precision of the calibration satisfied ANSI/HPS N13.30-1996 (see Reference 1) performance criteria and are documented in Appendix E.

In addition to the performance tests, a set of 5 validation counts were performed using a DOE manufactured NIST traceable Lung Set \# MFP-1-001. The validation counts Relative Bias and Relative Precision were tested to ANSI/HPS N13.30-1996 criteria. The results are documented in Appendix H. 


\section{COUNTING SYSTEM DESCRIPTION}

The Accuscan II system contains two broad energy BE type HpGe detectors used for both low and high energy photon counting. This would include Whole Body, High Energy Lung, Thyroid, and Low Energy Lung (although at this time the system is not calibrated for energies $<26 \mathrm{keV}$ ). The Canberra Apex-InVivo software system, supported by Genie 2000 Spectroscopy software, operates on a Dell Optiplex 780 PC. The counter system is located in a 9' 4 " high $\times 8^{\prime} 71 / 2$ " wide $\times 11^{\prime} 5^{\prime \prime}$ deep counting vault constructed of concrete and masonry blocks. The counting system is standup with the detectors scanning from head to foot for whole body counting. For Lung and Thyroid counting the detectors are stationary at fixed counting positions. Personnel are positioned in a counting shield (tub) constructed of 4 " of low background steel and face the detectors which are "shadow shielded" with both lead and copper. The counting vault is located on the INL site at HPL-1618. The detectors are cooled to $-185^{\circ} \mathrm{C}$ by an electrically refrigerated cryostat system. 


\section{CALIBRATION SOURCES AND PHANTOMS}

A NIST traceable lung set manufactured by the University of Cincinnati \# UCLL43AMEU \& UCSL43AMEU was used for both the energy and efficiency calibrations. The lung set contains uniformly distributed Am-241 and Eu-152 and is described in Appendix A. The source activity was decayed to the efficiency calibration date of April 5, 2011 by the Apex-InVivo software. The Apex-InVivo software also decayed the source for verification testing and displayed the results in gammas per second.

A Realistic Torso Phantom was used with the University of Cincinnati lung set to perform both the energy and efficiency calibrations. The phantom is described in Appendix I.

Validation counting of the calibration was performed using a NIST traceable DOE manufactured lung set \# MFP-1-001 containing Co-57, Co-60, CS-134 and Cs-137. This source certificate is documented in Appendix A. The source was decayed to the validation count date and is documented in Appendix B. 


\section{DETECTOR AND LYNX DIGITAL AMPLIFIER TESTING}

A Pole Zero was performed in accordance with MCP-3336 for both detectors during the I-131 calibration of March 1, 2011. No pole zero was performed for this calibration. 


\section{ENERGY/FWHM CALIBRATION}

On April 4, 2011 the CWI Realistic Torso Phantom was used together with the lung set \#UCLL43AMEU and UCSL43AMEU to obtain a calibration count to be used for the High Energy Lung (energy) calibration. The RMC-II counting stand and table were mounted in the Accuscan II counter with the pins removed. The Realistic phantom was placed on the table with the shoulder of the phantom between the v-ridges on the counter rearwall. The phantom chest plate was removed for the energy calibration. The Accuscan II detector housing was positioned such that the top of the detector housing was perpendicular to the junction of the phantom clavicle and sternum. This placed the center of the housing at the approximate midpoint of the phantom lungs. The calibration count was collected for 7228 seconds real time (2 hours).

The energy calibration was performed on April 5, 2011 for both the left, right and summed detectors. The energy calibration is documented in Appendix C. 


\section{EFFICIENCY CALIBRATION}

Subsequent to the High Energy Lung (energy) calibration, a calibration count was collected for the efficiency calibration. The CWI Realistic Torso Phantom was used together with the lung set \#UCLL43AMEU and UCSL43AMEU to obtain the calibration count. The RMC-II counting stand and table were mounted in the Accuscan II counter with the pins removed. The Realistic phantom was placed on the table with the shoulder of the phantom between the v-ridges on the counter rearwall. The phantom chest plate was reinstalled and the detector housing aligned such that the top of the housing was positioned perpendicular to the junction of the phantom clavicle and sternum. This placed the center of the housing at the approximate midpoint of the phantom lungs. The calibration count was collected for 7226.91 seconds real time (2 hours).

The efficiency calibration was performed on April 5, 2011 at 4:05 PM for the summed detector. The efficiency calibration is documented in Appendix D. 


\section{CALIBRATION COUNT REANALYSIS}

The calibration count collected 4/5/2011 at 2:02 PM was reanalyzed using the new energy and efficiency calibration dated 4/5/2011 8:18 AM and 4/5/2011 4:05 PM respectively. The results of the reanalysis indicated a Relative bias within 0.06 to -0.03 with an average bias of 0.012 . This result is well within the bias criteria of ANSI/HPS N13.30-1996. The results also indicated an excellent calibration. The summary result and supporting documentation is attached as Appendix E. 


\section{PERFORMANCE (VERIFICATION) TESTING}

Verification testing was conducted subsequent to the High Energy Lung efficiency calibration and was performed using the CWI Realistic Torso Phantom together with the lung set \#UCLL43AMEU and UCSL43AMEU to obtain a set (5) of verification counts. The RMC-II counting stand and Table were mounted in the Accuscan II counter with the pins removed. The Realistic phantom was placed on the table with the shoulder of the phantom between the v-ridges on the counter rearwall. The phantom chest plate was installed. The Accuscan II detector housing was positioned such that the top of the detector housing was perpendicular to the junction of the phantom clavicle and sternum. This placed the center of the housing at the approximate midpoint of the phantom lungs. The verification counts were collected for 600 seconds real time (10 minutes). The Apex software verification provision was used to collect the 5 counts on 4/6/2011. The Lung Set contained the radionuclides Am-241 and Eu-152. The result of the verification testing is summarized in Appendix F and indicates a Relative Bias between $+5.14 \%$ and $-14.80 \%$ (1086 keV peak of Eu-152). The average Relative Bias was $4.23 \%$. The Relative Precision indicated $1.03 \%$ to $24.56 \%$ (444 keV peak of Eu-152) with an average Relative Precision of 9.45\%. 


\section{MDA CALCULATIONS}

MDA testing was conducted on April 6, 2011. The Realistic Torso phantom was counted three times with a blank (no activity) set of lungs installed. The counts were performed using the ROUNPPGE.ASF file which contained the Detection Limit - Curie MDA equation $\left(4.65^{*} S_{b}+3\right) / K T$. The

INLPLANTLIB.NLB library was installed which contains the radionuclides of interest at the INL. The MDA summary and supporting documentation are attached as Appendix G. 


\section{VALIDATION TESTING}

High Energy Lung validation testing was conducted on 4/5/2011 and 4/6/2011 using the DOE Lung set \# MFP-1-001 and CWI Realistic Torso phantom. The RMC-II counting stand and Table were mounted in the Accuscan II counter with the pins removed. The Realistic phantom was placed on the table with the shoulder of the phantom between the v-ridges on the counter rearwall. The phantom chest plate was installed and the detector housing aligned such that the top of the housing was positioned perpendicular to the junction of the phantom clavicle and sternum. This placed the center of the housing at the approximate midpoint of the phantom lungs. The validation counts were collected for the normal chest counting time of 10 minutes. The MFP-1-001 phantom contained the radionuclides Co-57, Co-60, Cs-134 and Cs-137. The lung set also contained Mn-54; however, the activity was well below minimum testing detection levels. The results of the validation testing is summarized in Appendix $\mathrm{H}$ with a Relative Bias of $0.3 \%$ to $-3.6 \%$ and a Relative Precision of $1.1 \%$ to $18 \%$. Testing results are within the acceptance criteria of ANSI/HPS N13.30-1996. 


\section{SUMMARY AND CONCLUSION}

Final review of all calibration and verification count results confirmed that the high energy lung calibration created on April 5, 2011 for the Accuscan II counter is satisfactory for counting high energy photons when the detectors are positioned in-accordance-with Reference 2 (top of the detector housing is perpendicular to the junction of the individual's clavicle and sternum) - TEV-1273 - Basis for Positioning of the In Vivo Accuscan II Detectors. A summary of current calibration files for this counter geometry is shown in Table 1.

The INLPLANTLIB.NLB nuclide library file is the default for high energy lung counting. Non-systematic and systematic errors for the in vivo system are described and quantified in Reference 3 TEV-1275 (INL In Vivo System Counting Uncertainty).

The overall percent (\%) counting uncertainty using the Accuscan II for high energy lung counting is:

Total \% Uncertainty (Accuscan II) for High Energy Lung $=\sqrt{(\% \text { counting uncertainy })^{2}+(6.7)^{2}+(18.0)^{2}}$ 
Table 1. Calibration summary table.

\begin{tabular}{lcc}
\hline $\begin{array}{l}\text { Facility: } \\
\text { Counting System: } \\
\text { Counter Arrangement: }\end{array}$ & \begin{tabular}{l} 
Idaho National Laboratory, Building HPDL CFA-1618 \\
Accuscan II High Energy Lung Stationary Position \\
\multicolumn{1}{c}{ Detector }
\end{tabular} & $\begin{array}{c}\text { Accuscan II for High Energy Lung } \\
\text { Energy Calibration Date }\end{array}$ \\
\hline Accuscan II BE Detectors & $04 / 05 / 2011$ & Time \\
Efficiency Calibration: & & $8: 18$ AM \\
\hline \multicolumn{1}{c}{ Detector } & Energy Calibration Date & Time \\
\hline Accuscan II BE Detectors & $04 / 05 / 2011$ & $4: 05$ PM \\
\hline
\end{tabular}




\section{REFERENCES}

1. American National Standard, American National Standards Institute/Health Physics Society ANSI/HPS N13.30-1996, "Performance Criteria for Radiobioassay," 1996.

2. Idaho National Laboratory, Basis Document TEV-1273, "Basis for Positioning of the In Vivo Accuscan II Detectors," 2011.

3. Idaho National Laboratory, Basis Document TEV-1275, "INL In Vivo System Counting Uncertainty,” 2011. 
Appendix A

\section{Source Certificate}




\section{Appendix A \\ Source Certificate}

\section{UCLL43AMEU \& UCSL43AMEU}

Universlty of Clnelnnatl

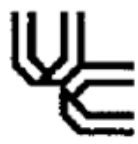

3 June, 1996

Mr. Patrick Rielly

U. S. Department of Energy

Lockheed Idaho Technologies Co.

Idaho National Engineering Lab

CF-690

Scoville, Idaho 83415

Dear Mr. Rielly,

\section{Calibnation Certificates for Calibnation. Phantom Lumes. POÄ C96-540439}

Enclosed please find the calibration certificates for the phantorn lungs UCLLA3AMEU and UCSL43AMEU containing ${ }^{24 I} \mathrm{Am}$ and ${ }^{152} \mathrm{Eu}$. There are four sets of certifieates: Each lung pluantom has one certificale for ${ }^{24} \mathrm{Am}$ and one for ${ }^{152} \mathrm{Ev}$. The final certified value of activity content is given on the third page of ench certificate in the blocks labeled comected activity of lung and amor in comected activity of hong. The correction is associated with the change in mass during foaming as a result of the loss of $\mathrm{CO}_{2}$. The pasameters adopled for lung density, volume, and uttenuation coefificient are the same as those used for the original Livemore National Laboratory lung phantoms and eited in ICRU publication \#44.

The surn of activity contaised in the large and small lung plantoms combined is $1,247.53 \mathrm{nCl}+10.9$ ! $\mathrm{nCi}^{24 \mathrm{i}} \mathrm{Am}$ and $543.05 \mathrm{nCi} \pm 1.45 \mathrm{nCi}{ }^{152} \mathrm{Eu}$. The total propagated uncertainty reported for the lung phanton represents 1 standard deviation and amounts to approximntely $1 \%$ to $2 \%$ of the total attivity. Factors for radianctive decay were oblnined from Table of lsolopes by Browne, Firestone, Shirley (1986). Copies of the certificales for the original madioactive materials used in construetion of the phantom have also been included to demonstrate tmeenbility of these solutions to the National Institute for Standares and Teelnnology.

The materials used to fabriente these lung pltantoms exactly duplicate the original materials used in the construction of the Livernnore National Liboratary phnntorn lungs, including the epoxy coating used on the surface of the lung phantoms. This epoxy resin conting rexuires a long time to become fully cured and hard. Curing is enlanced by removing the lung phantorns from the plastic bags used in shipping and allowing the phantoms to be open to the air. It is possible that this coating will become dannged with use and can easily be reapplied at any time in the future.

If you have any questions regarding the calibration phantom lungs or the calibration documentation, please do nat hesitate to contact me.

Very truly yours,<smiles>C=CC(C)(C)OC</smiles>

Herry B. Spit, Ph. D.

Associnte Professor of Nuclear Engineering

Health Physics Program Director

encl: Certificates 


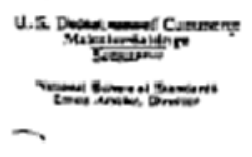

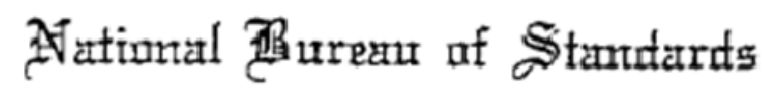
THIS IS PAOTOCOFY OF THE CERTACATE
U. ertifteat? SEPAMTE CDVER

Standard Reference Material $4370 \mathrm{C}$

Radioactivity Solution Standard

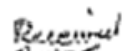

Radionucl1de Europien-152

Source identffication S370C

Soures doscription Liquid in 5-at, flame-sealed

glass arpoule (I)

Source nass $\quad 5.0338 \pm 0.0019$ grans (2)

Solutfon coaposition 277 ug zu per gran of $1 \mathrm{~K} \mathrm{HCl}$

13

Radioactivity concentracion

$9.390 \times 10^{4} \mathrm{Aq} \mathrm{g}^{-2}$

Rẹ̄erance time

1200 EST February 2, 1987

Overall uneartainty

1.1 parcent (3)

Photanenitting ixpurities

(activity ratio at refesence tire)

$25 \mathrm{Eu}^{2} / 15 \mathrm{Z}_{\mathrm{Eu}}(2.9 \pm 0.3) \times 10^{-3}$ (4)

Hale $14 \mathrm{fe}$

$13.55=0.06$ years (5)

Messuring inserument $4 \pi$ prossurized 1enfzacion-chanter
" $\mathrm{A}$ " provfously standardized by $4 \pi Y$ councing with the NBS $8 " x 8^{\prime \prime} \mathrm{NaI}(\mathrm{TL})$ erystals

This Standard Reference Matatial vas prepared in the Cancer for Radiation Research, Ionizing Radiation Division, Radiesetivity Group, Dale D. Hoppes, Group Leader.

Gaithersburg, 20899

Mareh, 1987
Stanlay O, Rasberry, Chie!

Office of Standard Referance Macerials

ANores on back 
(1) Approxinately tive millilters of solution. Aapouls speciffeacions:

\begin{tabular}{|c|c|}
\hline $\begin{array}{l}\text { body diamecer } \\
\text { wall ehickness } \\
\text { barium concent } \\
\text { lead oxide concent } \\
\text { ther heavy elements }\end{array}$ & $\begin{array}{l}16.5 \pm 0.5 \mathrm{~m} \\
0.60 \pm 0.06 \mathrm{~m} \\
\text { less than } 2.5 \text { percent } \\
\text { less than } 0.02 \text { percent } \\
\text { trace quantities }\end{array}$ \\
\hline
\end{tabular}

(2) The standart deviation 150.0019 grams based on mass pearurantents of is aupoules.

(3) The overall uncertaincy uas forned by taking three times the quadracic coabination oz̃ standart devintions of the aeian, of approxinations chereef, for the following:

a) 1987 Iontzation-chanber mearurementes on 17 sources

b) original fonizacion-chanbes calibracion using 7 sources

c) ratio of radiun zaforence sourees

d) solution atcenuation of $\gamma$-rays

e) graviretric nessurenents

f) impurities

8) decay correction

h) MaI(T1) measurenants on 6 sourcas

1) NaI(t1) datector effieiency

j) fluorescance yield Combined.

a.02 pareene

Q.08 percente

0.05 pereent

0.10 percent 0.15 percene 0.10 percerie 0.02 percent

0.02 percent 0.27 pereent 0.10 percent 0.37 percent

Overall $\frac{\times 3}{1.1}$ percent

(4) The linte of detection, exprossed as a pectencage of the gamasrayenission rate of the $1408-\mathrm{kaV}_{\text {gama }}$ rays exitted in the decay of europiumIS2, is

0.1 percent becueen 90 and $1900 \mathrm{keV}$,

provided that the impurity phatons ate separated In energy by $5 \mathrm{keV}$ or wore froa photons enited in the decay of europium-152 and europiun-154.

(5) NaS-neasured half-1ife value. NCRP Report No. 58, Znd edit1oa, 1985, p. 464 11ses a value of $13.4=0.1$ years.

For further information please contset M. S.M. Calhoun at (301) 975-5538 ot Dr. Bert M. Coursey at (30L) 975-5539.

$4370 C$ 

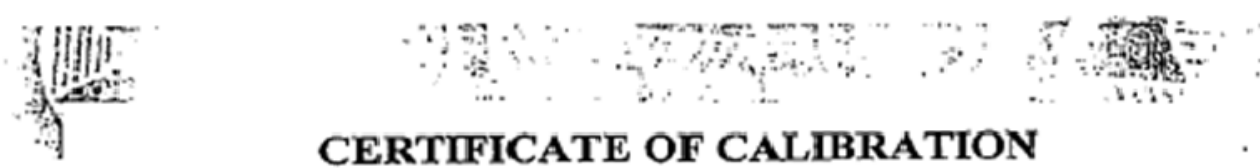

\section{CERTIFICATE OF CALIBRATION ALPHA STANDARD SOLUTION}

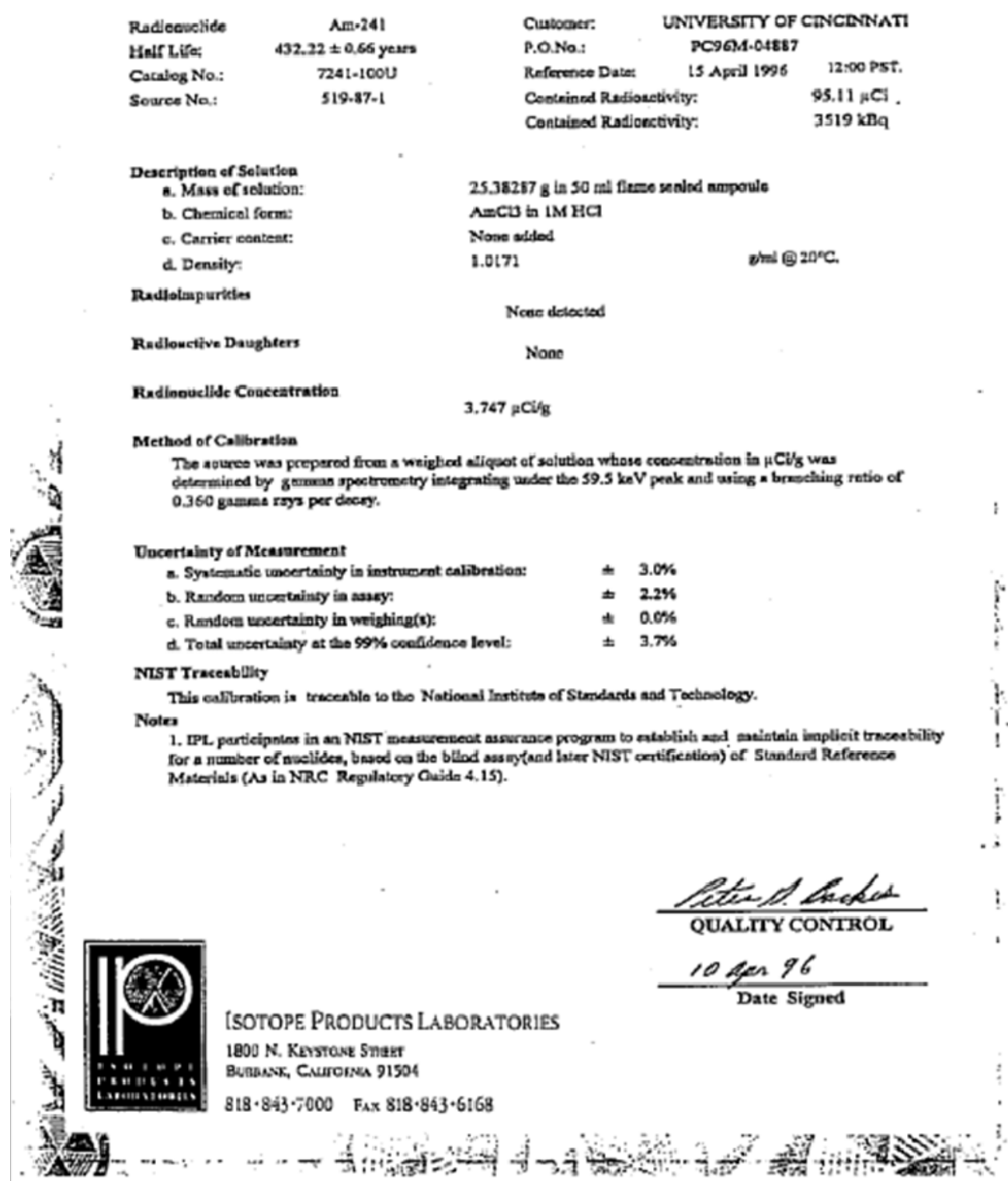


Re Univerily of Clichnat!

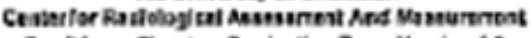

snat Lung Paselsen Preductios Form Versios 1.2

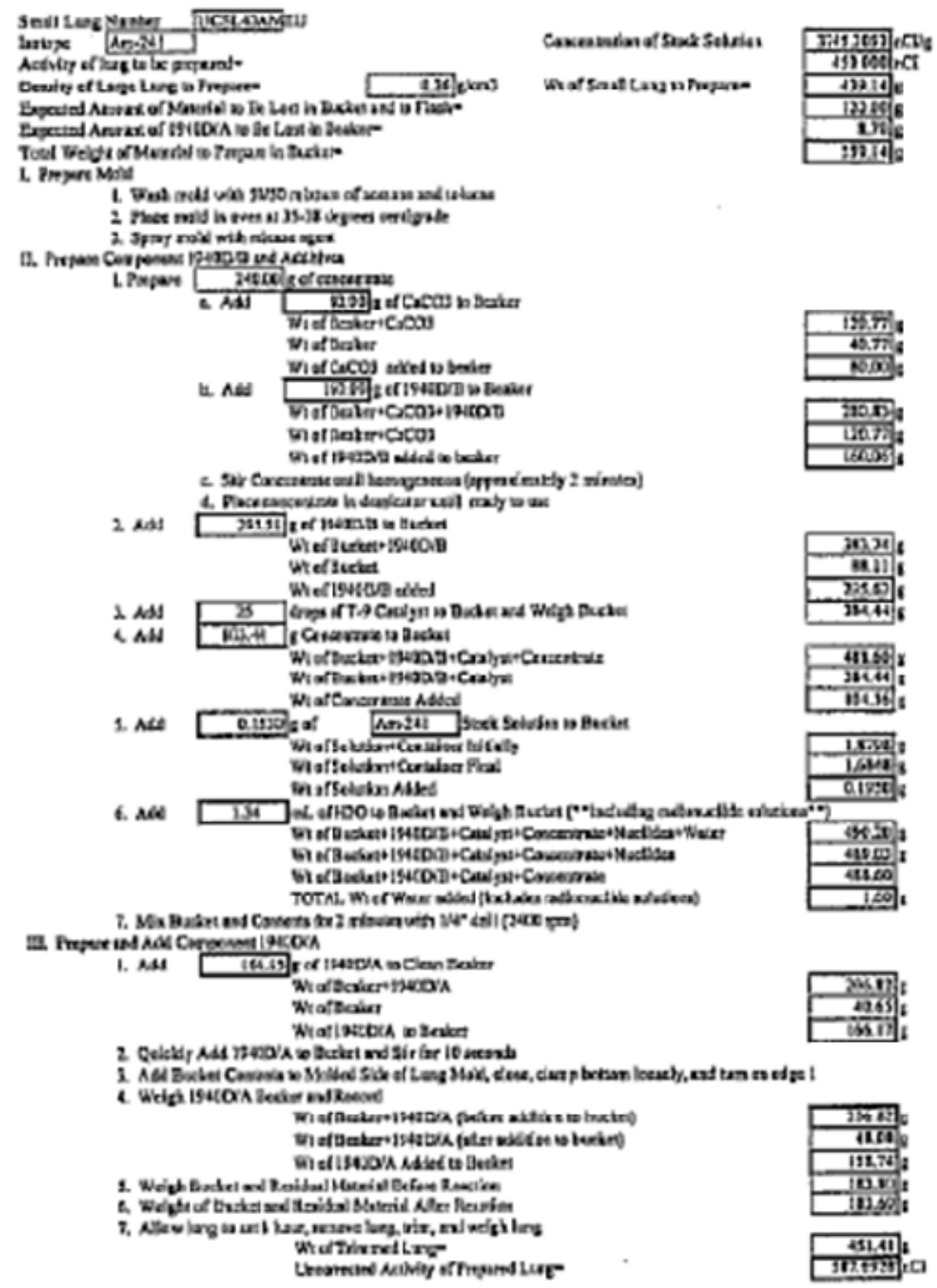

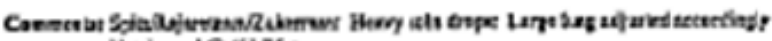
Untahred 0461.75 :

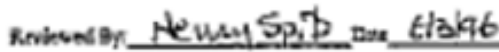

Letlawese

1131

sace 
The Unteasity ef Cinelertat

Cenler for RuColo jeol Assessmat And Masaremaal

Smal Lavg Plunism Produstos Form Vanise 1.9

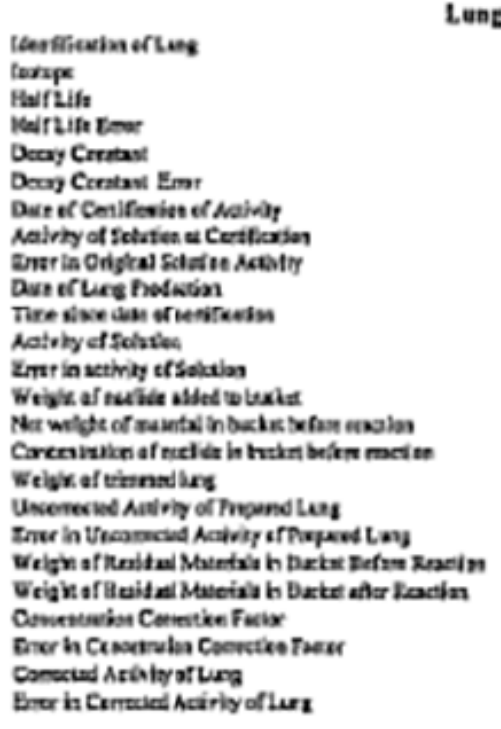

LenE Aethity Anaby's Stitet

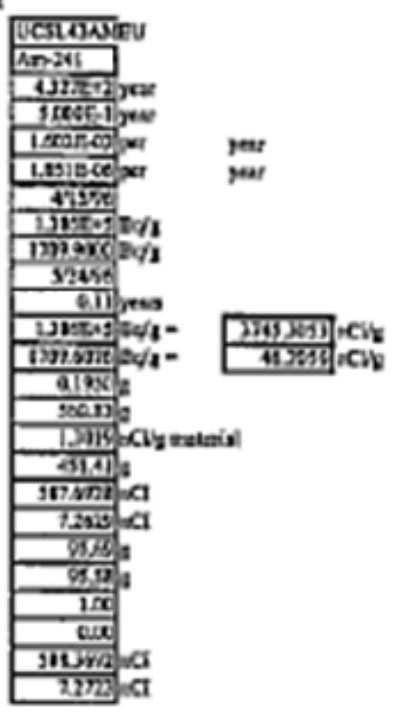

ucatavises 
Thu Unkerity af Chelona!

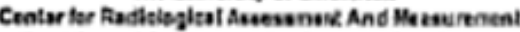

Irrat ung pharbaen Droduction Fsen Vercka 19

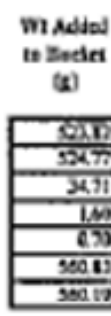

Qmetiy Aminca Worktest

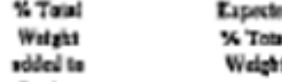

Accogtel

Toal

[vm]

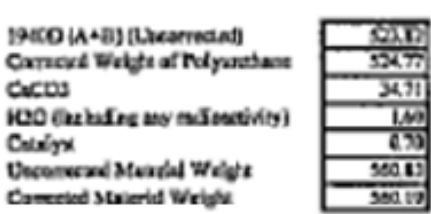

torbet

(2.9.

\begin{tabular}{|l|}
\hline a.j \\
\hline 0.21 \\
\hline
\end{tabular}

\begin{tabular}{|c|}
\hline 1215 \\
\hline 0125 \\
\hline
\end{tabular}

0.12

Desaly

Cascratuba Cerwala foces

Wis H

Wh 2 C

W) $* 5$

Wis 0

Wis 6 .

Wv $16 \mathrm{M}$

Wv5 80

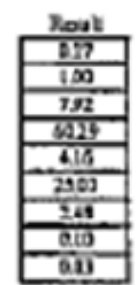

Ciarded

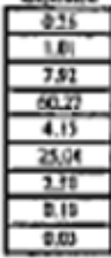

काm?

Censast Arahly sttureto

Emr in Cenetied Activing eftura (rC)

$72 \pi$

astace

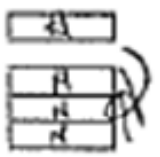

Bundart Geaviss io

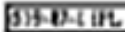

Ucstamexis

11:51

wast 
Mne Univenty et Clatinuts

Center fer Rathiegleal Lesessmest And Mevesement

3nol Luag Phatton Pwedexten Feon Verise 1.9

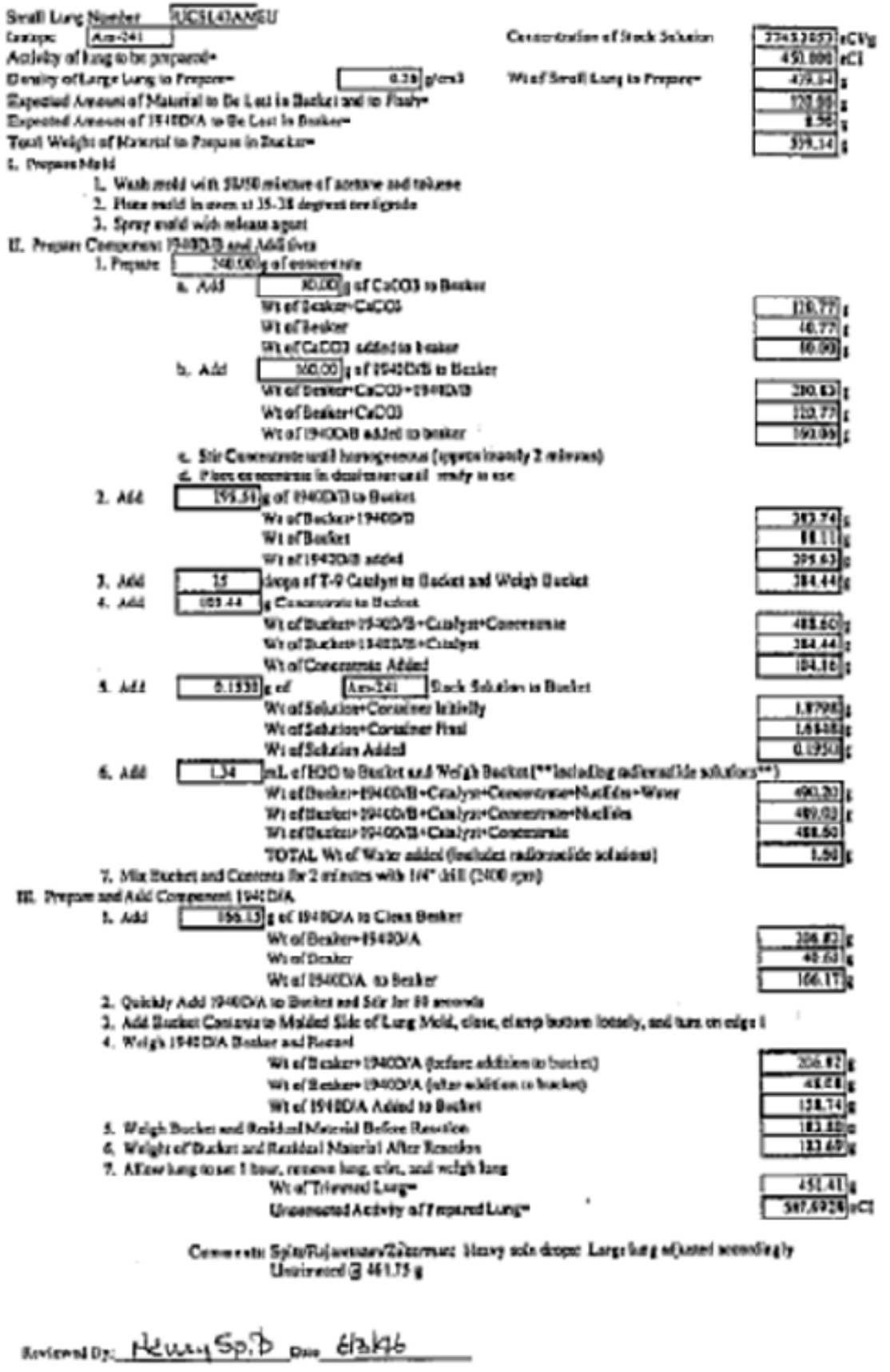

verusumst

1131

ersols 


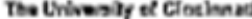

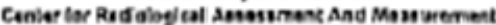

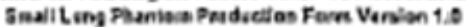

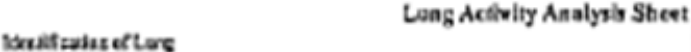

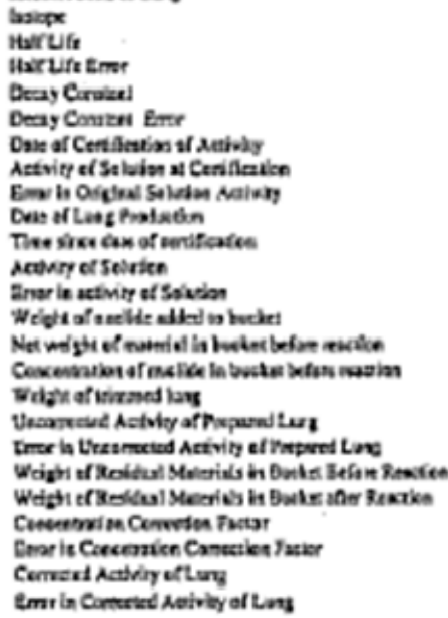

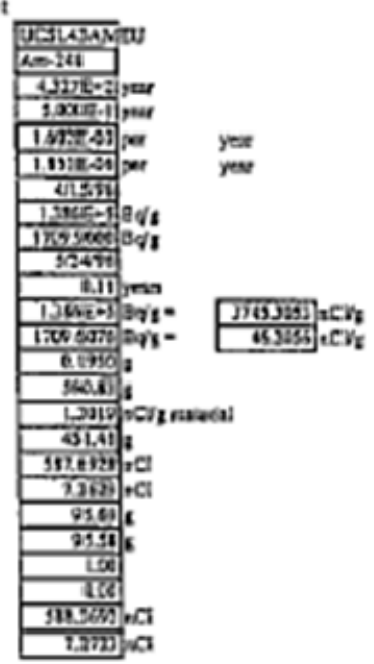


Do Uateenity at Clecinnat

Camar toe Radfotoglat Asuesunent A ne Vasuarenent Snall Ling Pasuton Frobetion form Veralon 1.8

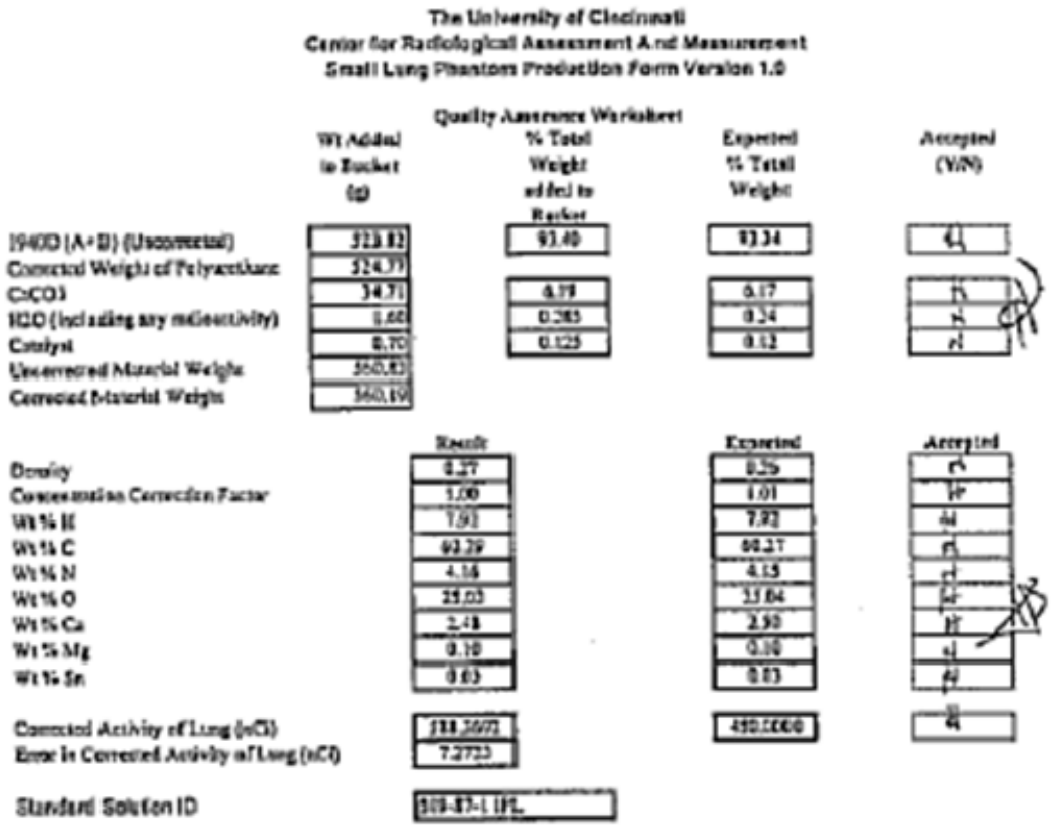

veslasurns

its,

409: 
The Univeriy at Cheinnet

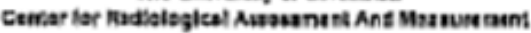

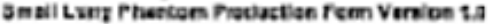

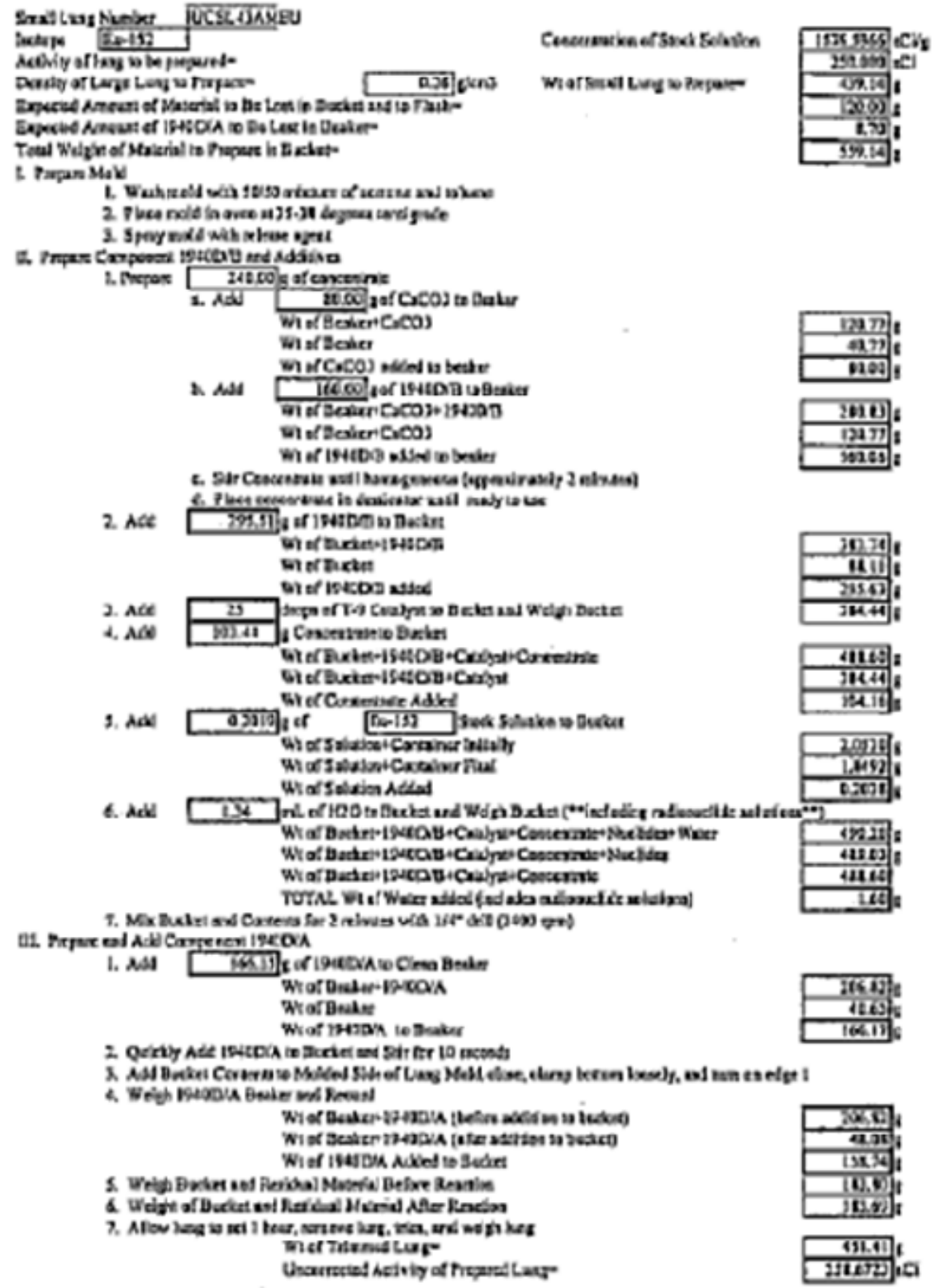

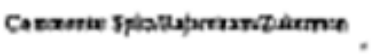

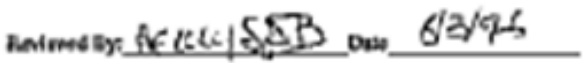

vestoeuns

1151

cones 
Tho Uninerely el Chelvast

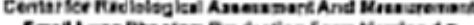

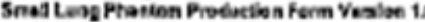

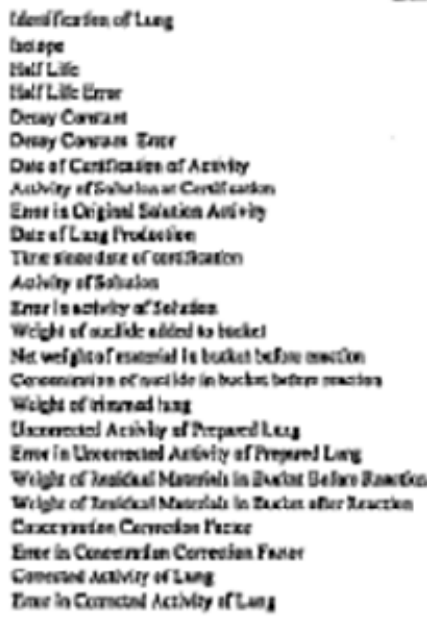

Lesa Acthity Analyn's Sheet

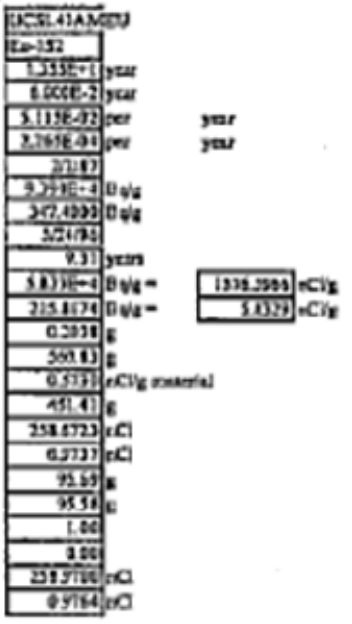




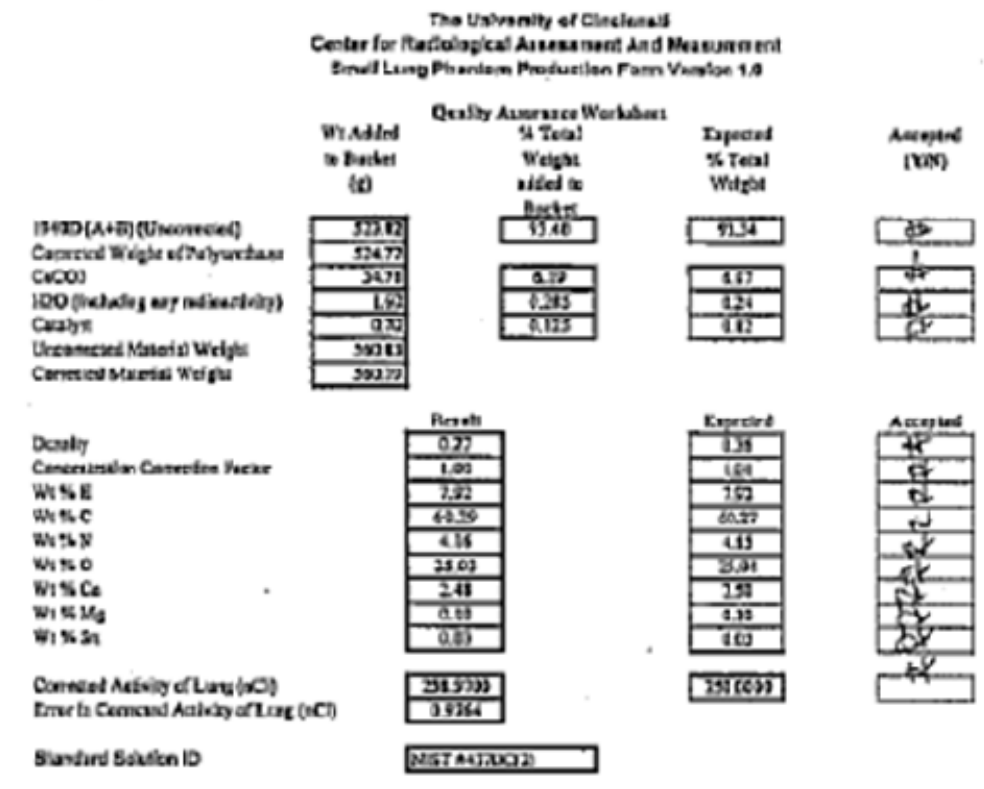

UCS,Aneuxs

ins! 
Tha Uetennity of Ciactin st:

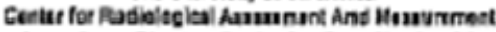

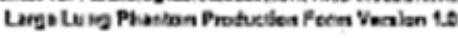

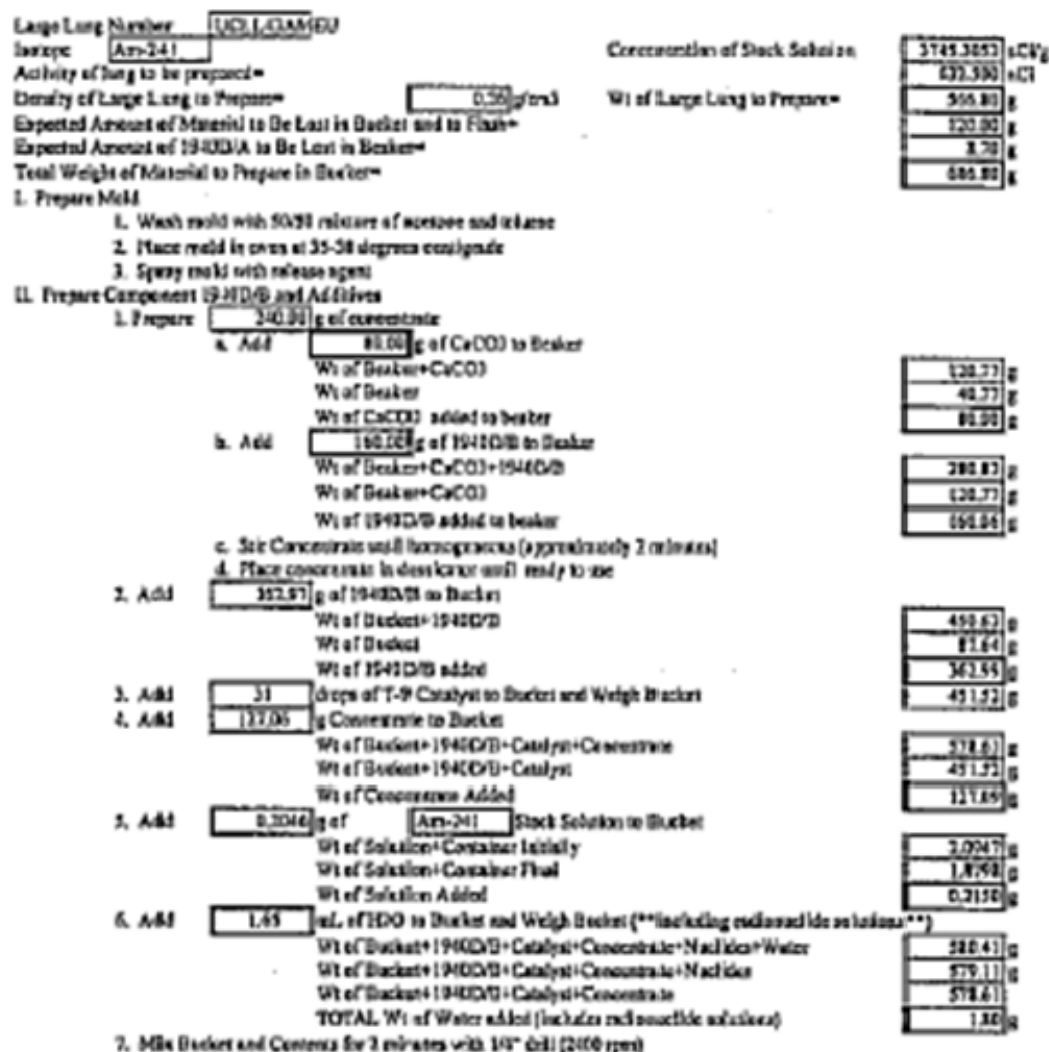

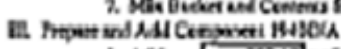

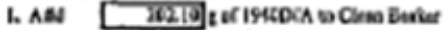

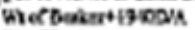

ติ

Whor 1200 'A o Peater

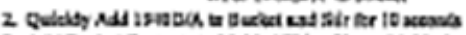

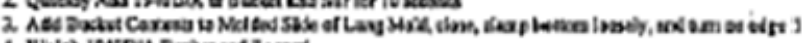

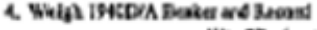

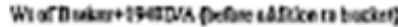

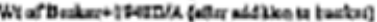

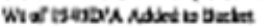

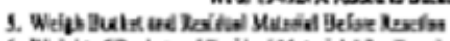

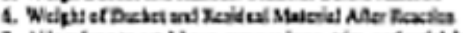

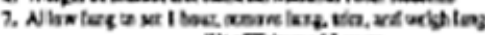
Wearticared tore

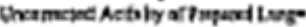

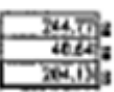

a.18.

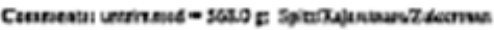

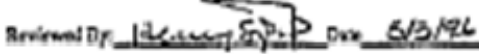

uculasure

1100 
To. Univeaby et Cinchnot

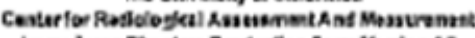

Large Lurg Pasiton Produrlos Fern Viales 10

thetillotias of Luas

Guag Activity Asalyali Sheal

soope

War uift

Dear Ceresan

Dea) Cesan Pro:

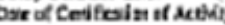

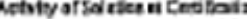

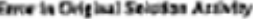

Dre of Las frodertan

Tims siret Les of warif cosin

Acivity or 3xifen

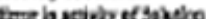

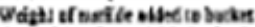

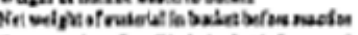

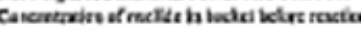

Whipi ef aiseod heg

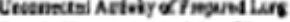

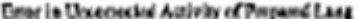

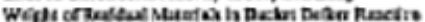

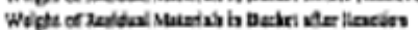

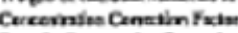

Eme is Crecentis Creation Focas

Cecrenes Aatho aftion

Ene ia Coneet Actaty arture

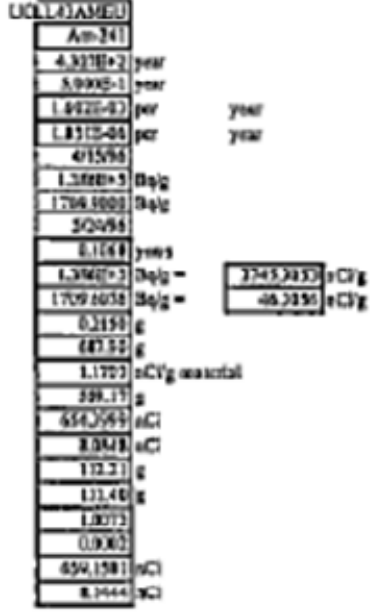

valouras

Ints

ธอง 


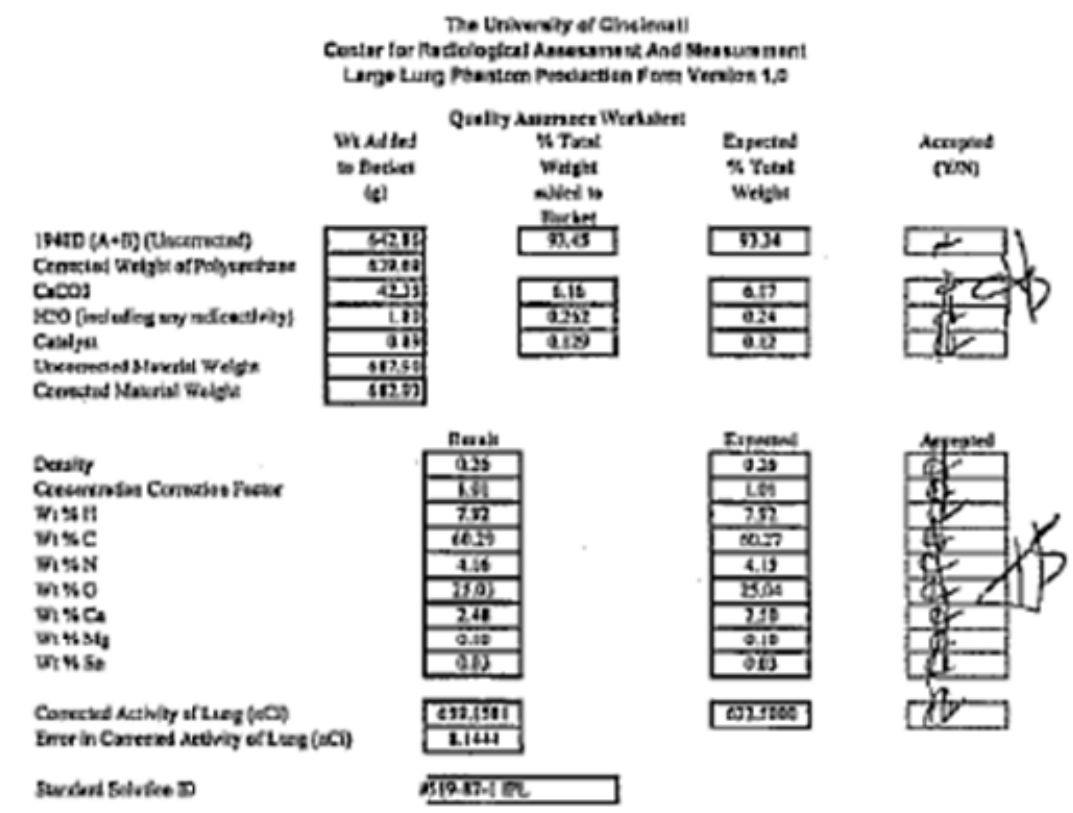

vacusnux:s 
The Unlarevity at Chetionu

Centir lar Redblegkal Anesenest And Manurenost

Larpe Lueg Phuetoen Brodgetisn Forn Vorien 1.9

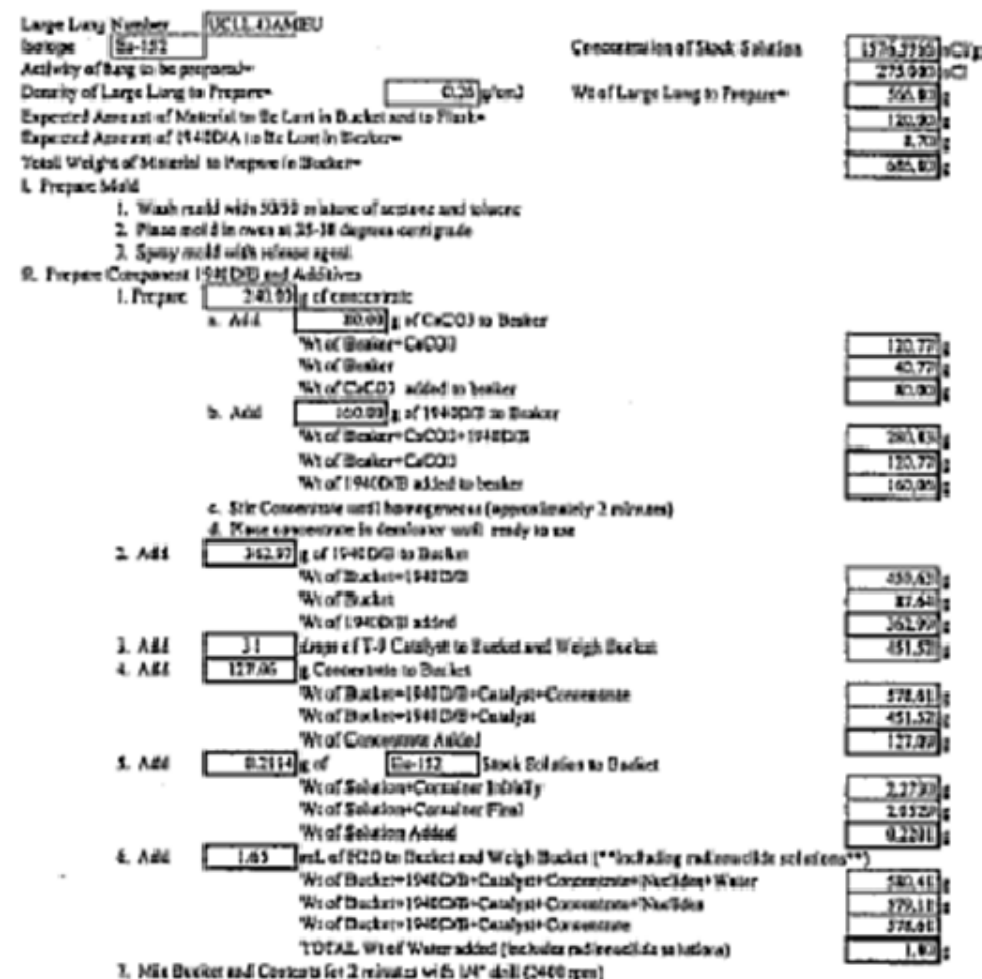

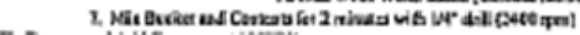

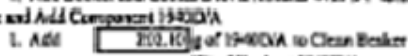
Wi ef Bealem-tsicos Wi af Destr

Whaf $1500 \mathrm{~A}$ แล $3 \mathrm{ab}$

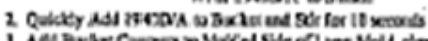

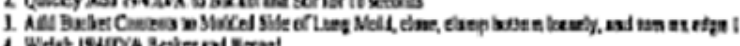

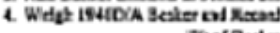

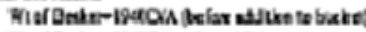

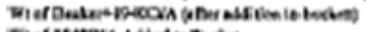

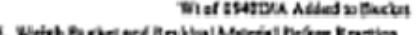

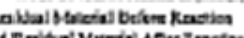

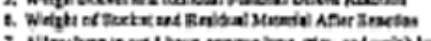

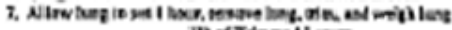

Wh efThened Lons

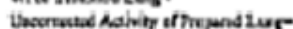
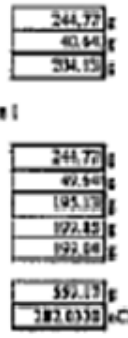

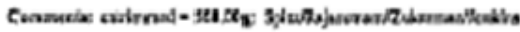

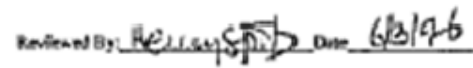

UsclasenXus

$11 \times 42$
6093 
The Univashy of Ontienat

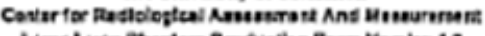
Lirge Lusa Mhantem Prafustlon Pern Vealan 10

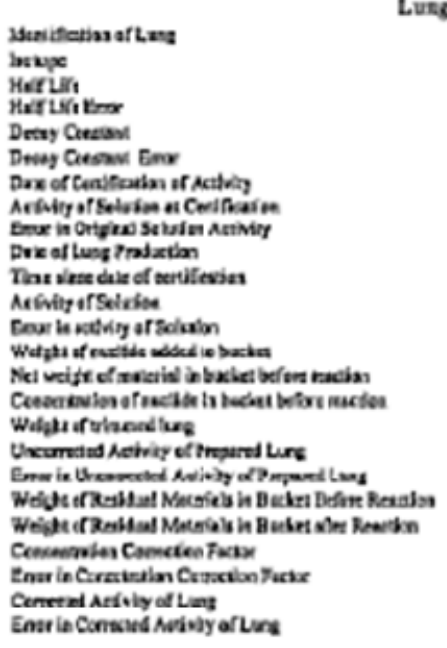

Lung Activity Anabyls Sheet

\begin{tabular}{|c|c|c|}
\hline \multicolumn{3}{|c|}{ 1.9ANDप } \\
\hline C.15 & & \\
\hline 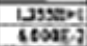 & $y=x$ & \\
\hline गागहै। & per & $y e$ \\
\hline 220182 & per & jor \\
\hline एक & & \\
\hline 300004 & aqa & \\
\hline भार्टव & $a w$ & \\
\hline 3206 & & \\
\hline $25 \times 9$ & years & \\
\hline towent & byan & {$[5 \times 0.505]_{1} \mathrm{CV}_{2}$} \\
\hline 20.017 & $9 v 2=$ & $2.02]=\mathrm{Cl}_{3}$ \\
\hline Q.20 & $\mathrm{s}$ & \\
\hline क्रिक & c & \\
\hline asen & iCVyeresta & \\
\hline$m 01$ & $\mathrm{~s}$ & \\
\hline 29218 & aca & \\
\hline L.2E & loci & \\
\hline [12]1 & & \\
\hline IILet & 8 & \\
\hline $1.2 \mathrm{n}$ & & \\
\hline c200 & & \\
\hline 212a01 & ea & \\
\hline t.zsit & a & \\
\hline
\end{tabular}

vchoeurs 


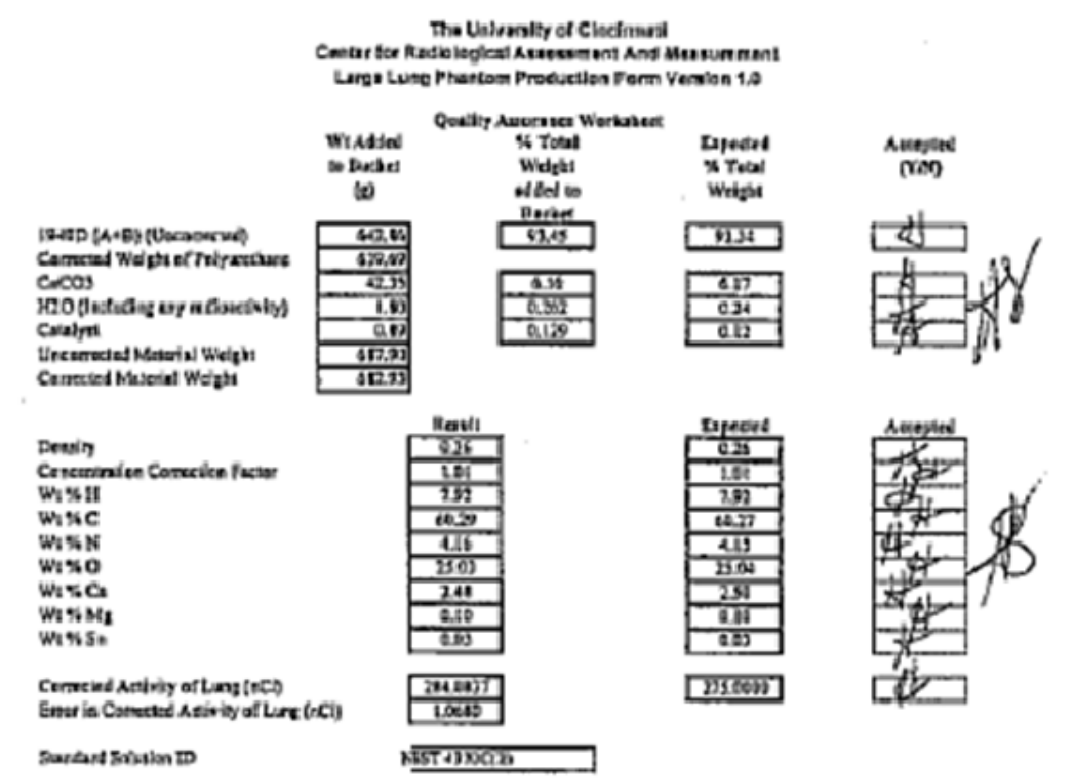

\footnotetext{
vacustux $\times 1$ s

$15 e$
} 


\title{
Certified Zieference Atlaterial
}

Certificate of Traceability

\section{U.S. Department of Energy \\ Radiological and Environmental Sciences Laboratory}

\author{
Standard Identification: MFP-1-001
}

Radionuclide

Activity

$\begin{array}{ll}\mathrm{Mn}-54 & 5.44+/-0.04 \mathrm{E} 2 \mathrm{nCi} \\ \mathrm{Co}-57 & 4.39+/-0.04 \mathrm{E} 3 \mathrm{nCi} \\ \mathrm{Co}-60 & 3.53+/-0.03 \mathrm{E} 2 \mathrm{nCi} \\ \mathrm{Cs}-134 & 3.92+/-0.03 \mathrm{E} 2 \mathrm{nCi} \\ \mathrm{Cs}-137 & 4.56+/-0.04 \mathrm{E} 2 \mathrm{nCi}\end{array}$

This Certified Reference Material (CRM) contains a known quantity of racionuclides in a stable and homogeneous matrix. This material is intended for the calibration of instruments and for the verification of the accuracy and precision of analytical radiochemistry measurements. The activities listed for each radionuclide are traceable to NIST through an unbroken chain of comparisons, all having stated uncertainties $(k=1)$ calculated according to ISO and NIST Guidelines.

This material was prepared by the Radiological and Environmental Sciences Laboratory (RESL). RESL maintains direct traceability to NIST through successful participation in the NIST/RESL Radiological Traceability Program and is accredited to ISO 17043 as a Performance Testing Provider and ISO 17025 as a Chemical Testing Laboratcry by The American Association for Laboratory Accreditation.

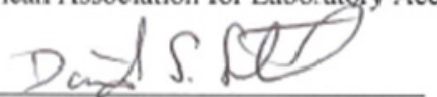

David S. Sill

Senior Technical Manager - Chemistry

April 22, 2005

Reference Date

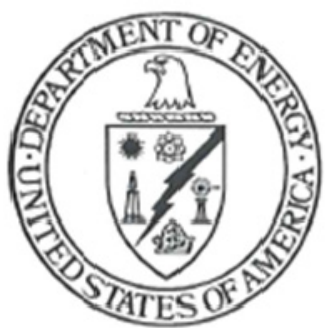


Supplemental Information

$\begin{array}{llll} & \text { Left Lung } & \text { Right Lung } & \text { Total } \\ \text { Density }(\mathrm{g} / \mathrm{cm} 3) & 0.269+/-0.002 & 0.259+/-0.001 & 0.263+/-0.001 \\ & & & \\ \text { Activity }(\mathrm{nCi}) & & & \\ \text { Mn-54 } & 2.50+/-0.04 \mathrm{E} 2 & 2.94+/-0.01 \mathrm{E} 2 & 5.44+/-0.04 \mathrm{E} 2 \\ \text { Co-57 } & 2.02+/-0.03 \mathrm{E} 3 & 2.370+/-0.011 \mathrm{E} 3 & 4.39+/-0.04 \mathrm{E} 3 \\ \text { Co-60 } & 1.62+/-0.03 \mathrm{E} 2 & 1.91+/-0.01 \mathrm{E} 2 & 3.53+/-0.03 \mathrm{E} 2 \\ \text { Cs-137 } & 1.80+/-0.03 \mathrm{E} 2 & 2.12+/-0.01 \mathrm{E} 2 & 3.92+/-0.03 \mathrm{E} 2 \\ \text { Cs-137 } & 2.09+/-0.03 \mathrm{E} 2 & 2.47+/-0.01 \mathrm{E} 2 & 4.56+/-0.04 \mathrm{E} 2\end{array}$

Reference Date April 22, 2005 


\title{
DOE Source Certificate I-125 BEA Thyroid 002
}

\section{Certifíed גeference Atlaterial}

Certificate of Traceability

\author{
U.S. Department of Energy \\ Radiological and Environmental Sciences Laboratory
}

Standard Identification: I-125 BEA Thyroid 002

Radionuclide

Activity

I-125

$(1.33 \pm 0.02) \mathrm{E} 2 \mathrm{nCi}$

This Certified Reference Material (CRM) contains a known quantity of radionuclides in a stable and homogeneous matrix. This material is intended for the calibration of instruments and for the verification of the accuracy and precision of analytical radiochemistry measurements. The activities listed for each radionuclide are traceable to NIST through an unoroken chain of comparisons, all having stated uncertainties $(k=1)$ calculated according to ISO and NIST Guidelines,

This material was prepared by the Radiological and Environmental Sciences Laboratory (RESL). RESL maintains direct traceability to NIST through successful participation in the NIST/RESL Radiological Traceability Program and is accredited to ISO 17043 as a Performance Testing Provider and ISO 17025 as a Chemical Testing Laboratory by The American Association for Laboratory Accreditation.

David S. Sill

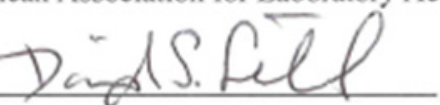

Senior Technical Manager - Chemistry

February 28, 2011

Reference Date

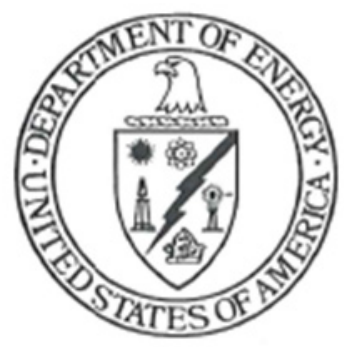


Supplemental Information

\section{I-125 BEA Thyroid 002}

Final weight of vial with sticker and tape: 38.885 grams

Weight of solution in vial: $29.955+/-0.002$ grams

Solution Used: 1-125-80943-370

Solution in vial: Solidified with 0.432 grams of WaterWorks Crystal SP400.

Half life: 59.40 days

Certificate and dilution information attached.

Prepared by Guy Backstrom, 2/28/2011 
Appendix B

\section{Source Decay}




\section{Appendix B Source Decay \\ UCLL43AMEU \& UCSL43AMEU Lung Set}

\begin{tabular}{cccc} 
& \multicolumn{2}{c}{ Eu-152, Am-241 Source Decay } \\
& Source ID - UCLL43AMEU, UCSL43AMEU \\
& Accuscan II Calibration for High Energy Lung Geometry \\
Reference Date & Calibration date & Decay Time in Days \\
$5 / 24 / 19960: 00$ & $4 / 5 / 20110: 00$ & 5429.00 & \\
& & & \\
& & & \\
Radionuclide & Half Life (days) & Initial Activity (uCi) & New Activity (uCi) \\
Eu-152 & 4946 & 0.5431 & 0.2538 \\
Am-241 & 157760.3 & 1.2475 & 1.2181
\end{tabular}




\section{DOE Manufactured Lung Set \# MFP-1-001 Decay}

DOE Manufactured Source MFP-1-001
$4 / 4 / 2011$

$4 / 5 / 2011$

1
Reference

Date

Current Date

Elapsed Time

Identity

Co-60

Cs-137

Cs-134

Cs-134

Mn-54

Co-57
1173.2 1332.5

661.7

123

475

563

604

795.8

801.9

122.06

834.8
Reference

Activity

(nCi)

161

161

398

53.2

53.2

53.2

53.2

53.2

53.2

16.9

85.6

99.9

100

85.1

2.47

1.46

8.38

97.6

85.4

8.73

T1/2

Days

$\mathrm{G} / \mathrm{n}$

Reference
GPS

37

1925

1925

37

$5.95 \mathrm{E}+03$

$5.96 \mathrm{E}+03$

$1.61 \mathrm{E}+02$

$1.61 \mathrm{E}+02$

$\begin{array}{llllll}4.4 & 100 & 312.5 & 37 & 1.63 E+02 & 4.40 E+00\end{array}$


Appendix C

\section{Energy/FWH and Low Tail Calibration Documentation}




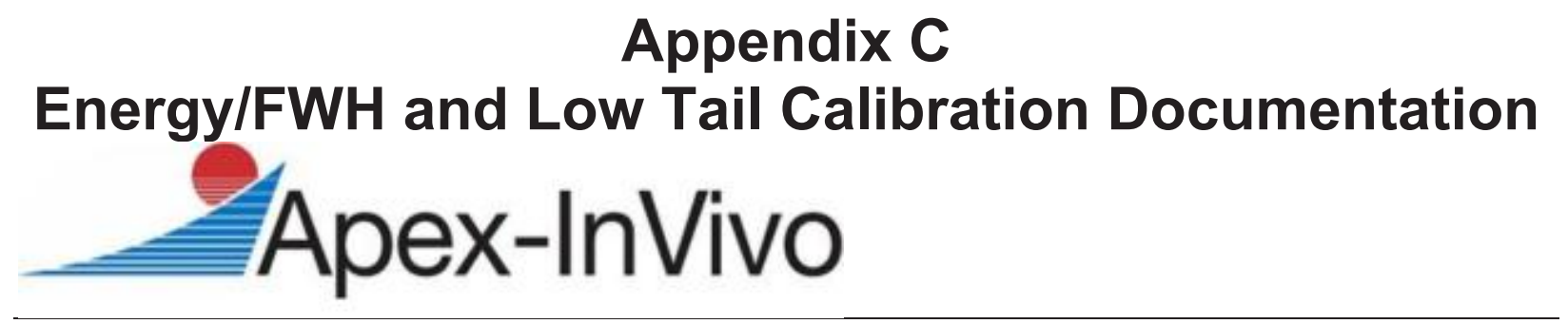

Energy Calibration

\section{Calibration Information}

$\begin{array}{ll}\text { Calibration Title } & \text { Energy Calibration (HE Lung) 4/5/2011 8:17 AM } \\ \text { Calibration Date } & 4 / 5 / 2011 \text { 8:17:58 AM } \\ \text { Performed By } & \text { David Georgeson } \\ \text { Count Used } & \text { Calibration Count 4/4/2011 4:49 PM } \\ \text { Is Default } & \text { Yes }\end{array}$

\section{Counter Information}

$\begin{array}{ll}\text { Counter Name } & \text { Accuscan II } \\ \text { Counter Location } & \text { Battelle }\end{array}$

\section{Detector Group Information}

Detector Group Name

Calibration Source

Left

Analysis Sequence File

Am241/Eu152 CWI Lung Set

Nuclide Library

QCPEAKBE.ASF

Peak Search Library

$$
\begin{aligned}
& \text { Energy Calibration Coefficients } \\
& \qquad \begin{aligned}
\text { Energy } & =-0.236715+0.461979 * \mathrm{ch}+1.14186 \mathrm{e}-08 * \mathrm{ch}^{\wedge} 2 \\
\mathrm{FWHM} & =1.22288+0.0195807 * \mathrm{E}^{\wedge} 1 / 2 \\
\text { LOW TAIL } & =0.864533+0.000833452 * \mathrm{E}
\end{aligned}
\end{aligned}
$$

\begin{tabular}{|c|c|c|}
\hline \multicolumn{3}{|c|}{ Energy Calibration Results Table } \\
\hline Centroid Channel & Centroid Error & Energy (keV) \\
\hline 57.49 & 0.19 & 26.34 \\
\hline 129.53 & 0.02 & 59.54 \\
\hline 264.11 & 0.01 & 121.78 \\
\hline 530.10 & 0.03 & 244.70 \\
\hline 745.63 & 0.02 & 344.28 \\
\hline 890.15 & 0.04 & 411.10 \\
\hline 961.30 & 0.07 & 443.97 \\
\hline 1686.35 & 0.06 & 778.00 \\
\hline 1877.80 & 0.08 & 867.40 \\
\hline 2086.97 & 0.05 & 964.10 \\
\hline 2350.42 & 0.11 & 1086.00 \\
\hline 2407.45 & 0.05 & 1112.00 \\
\hline 2625.97 & 0.20 & 1213.00 \\
\hline 2812.33 & 0.23 & 1299.00 \\
\hline 3047.92 & 0.05 & 1408.00 \\
\hline
\end{tabular}

Approved by: 
Date:

User: David Georgeson

Energy Calibration

\section{Energy}

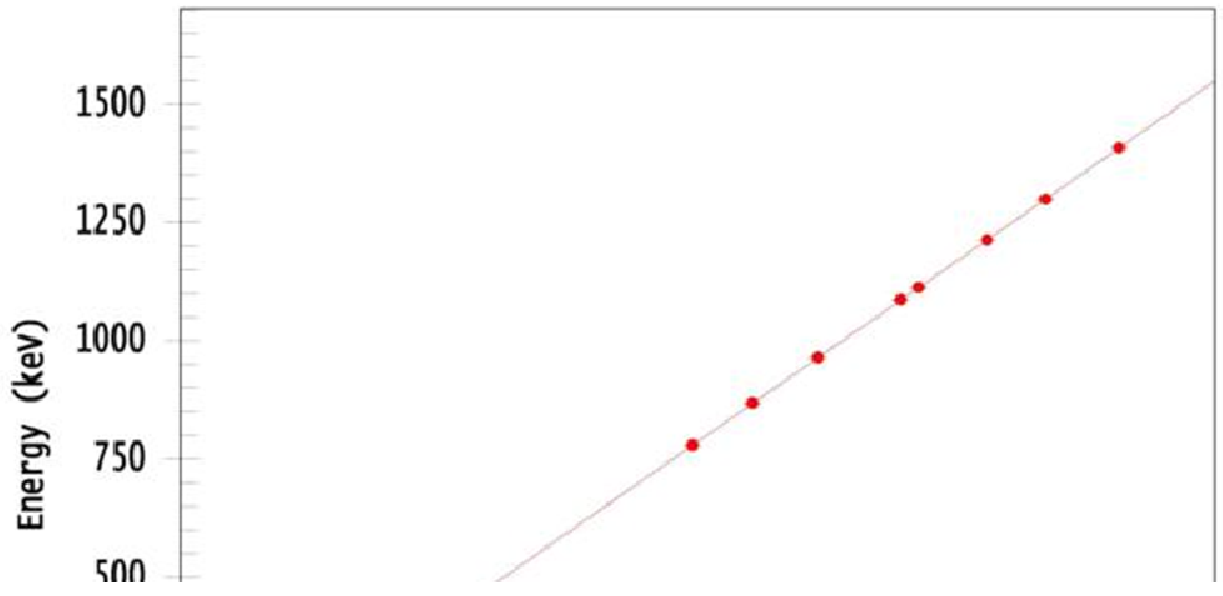

Energy Calibration Results Table

\begin{tabular}{rrr} 
Energy $(\mathbf{k e V})$ & FWHM Channels & FWHM Error \\
\cline { 2 - 3 } 26.34 & 2.61 & 0.47 \\
\hline 59.54 & 3.07 & 0.04 \\
\hline 121.78 & 3.14 & 0.02 \\
\hline 244.70 & 3.21 & 0.07 \\
\hline 344.28 & 3.38 & 0.04 \\
\hline 411.10 & 3.09 & 0.06 \\
\hline 443.97 & 3.80 & 0.14 \\
\hline 778.00 & 3.75 & 0.12 \\
\hline 867.40 & 4.07 & 0.17 \\
\hline 964.10 & 4.11 & 0.10 \\
\hline 1086.00 & 3.65 & 0.23 \\
\hline 1112.00 & 4.28 & 0.12 \\
\hline 1213.00 & 3.22 & 0.31 \\
\hline 1299.00 & 4.00 & 0.49 \\
\hline 1408.00 & 4.59 & 0.09 \\
\hline
\end{tabular}

User: David Georgeson

Energy Calibration

\section{Shape}

FWHM Low Tail

15.0 
User: David Georgeson 


\section{Energy Calibration}

\section{Calibration Information}

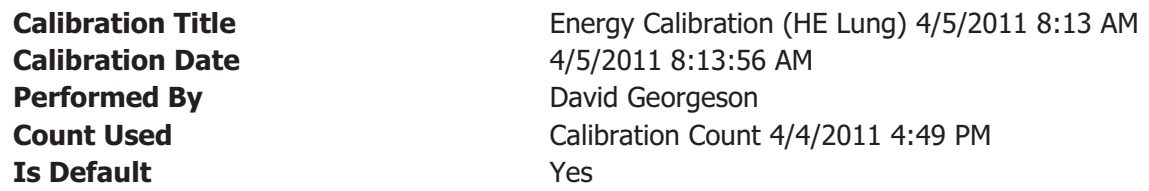

\section{Counter Information}

$\begin{array}{ll}\text { Counter Name } & \text { Accuscan II } \\ \text { Counter Location } & \text { Battelle }\end{array}$

\section{Detector Group Information}

$\begin{array}{ll}\text { Detector Group Name } & \text { Right } \\ \text { Calibration Source } & \text { Am241/Eu152 CWI Lung Set } \\ \text { Analysis Sequence File } & \text { QCPEAKBE.ASF } \\ \text { Nuclide Library } & \\ \text { Peak Search Library } & \end{array}$

\section{Energy Calibration Coefficients}

$$
\begin{aligned}
\text { Energy } & =-0.244432+0.461855 * \mathrm{ch}+3.89635 \mathrm{e}-08 * \mathrm{ch} \wedge 2 \\
\text { FWHM } & =0.577324+0.0294204 * \mathrm{E}^{\wedge} 1 / 2 \\
\text { LOW TAIL } & =0.726181-0.000167099 * \mathrm{E}
\end{aligned}
$$

\section{Energy Calibration Results Table}

\begin{tabular}{rrr} 
Centroid Channel & Centroid Error & Energy $(\mathbf{k e V})$ \\
\cline { 2 - 3 } 57.61 & 0.09 & 26.34 \\
\hline 129.57 & 0.02 & 59.54 \\
\hline 264.23 & 0.01 & 121.78 \\
\hline 530.24 & 0.02 & 244.70 \\
\hline 745.81 & 0.01 & 344.28 \\
\hline 890.50 & 0.03 & 411.10 \\
\hline 961.61 & 0.03 & 443.97 \\
\hline 1686.55 & 0.04 & 778.00 \\
\hline 1878.09 & 0.04 & 867.40 \\
\hline 2087.36 & 0.03 & 964.10 \\
\hline 2350.75 & 0.16 & 1086.00 \\
\hline 2407.67 & 0.03 & 1112.00 \\
\hline 2626.15 & 0.23 & 1213.00 \\
\hline 2813.14 & 0.30 & 1299.00 \\
\hline 3048.21 & 0.04 & 1408.00 \\
\hline & &
\end{tabular}

Approved by:

Date: 


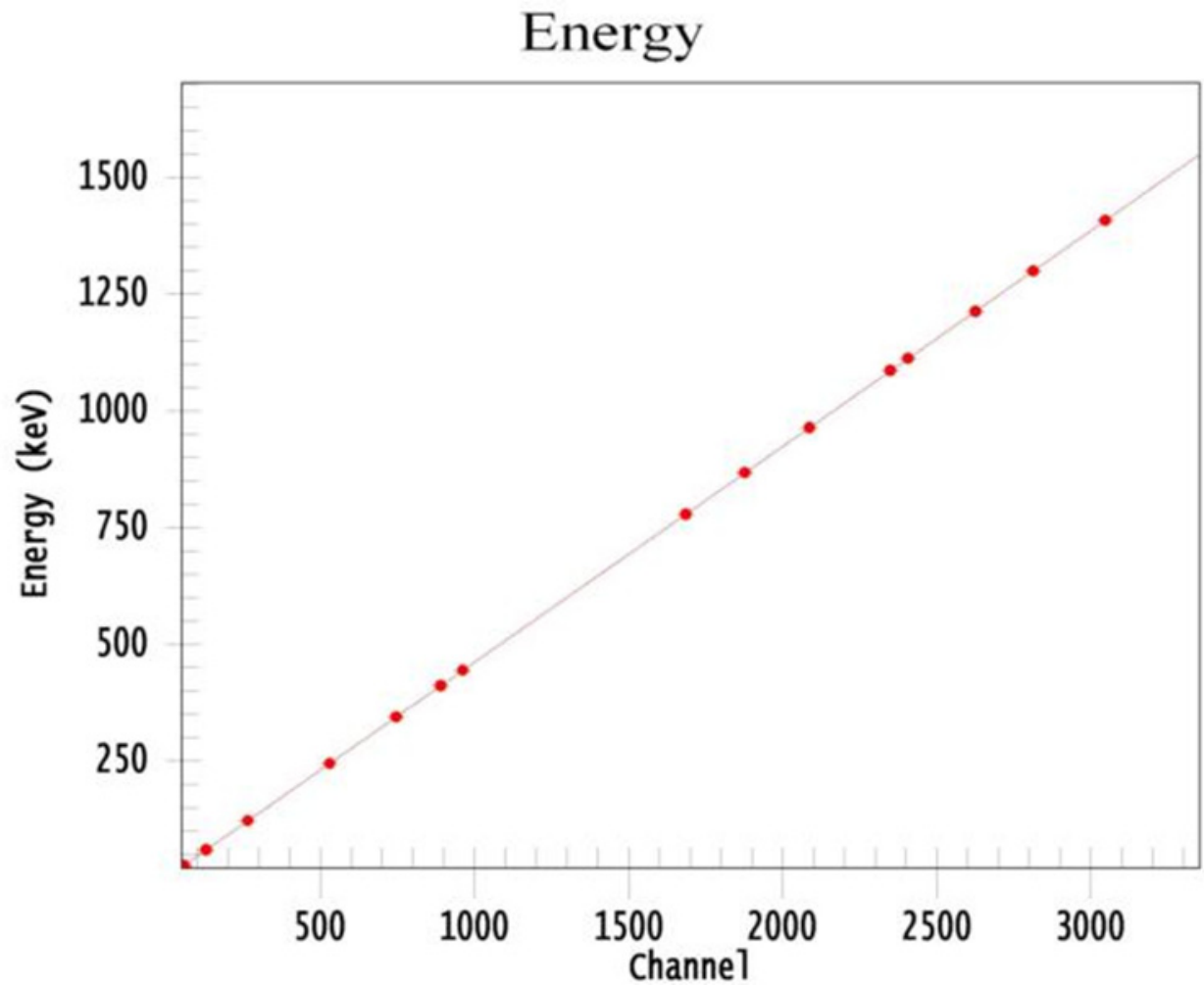

\section{Energy Calibration Results Table}

\begin{tabular}{rrrrr}
\hline Energy $(\mathbf{k e V})$ & FWHM Channels & FWHM Error & TAIL Channels & $\frac{\text { TAIL Error }}{0.37}$ \\
\cline { 2 - 5 } 26.34 & 1.61 & 0.27 & 0.72 & 1.16 \\
\hline 59.54 & 1.82 & 0.03 & 1.67 & 0.12 \\
\hline 121.78 & 1.97 & 0.02 & 1.60 & 0.18 \\
\hline 244.70 & 2.21 & 0.04 & 2.09 & 0.19 \\
\hline 344.28 & 2.39 & 0.02 & 6.42 & 0.18 \\
\hline 411.10 & 2.63 & 0.07 & 1.49 & 1.00 \\
\hline 443.97 & 2.46 & 0.06 & 2.28 & 0.20 \\
\hline 778.00 & 2.99 & 0.08 & 2.04 & 0.48 \\
\hline 867.40 & 2.85 & 0.08 & 18.97 & 0.48 \\
\hline 964.10 & 3.31 & 0.06 & 1.78 & 1.00 \\
\hline 1086.00 & 3.06 & 0.36 & 2.69 & 0.86 \\
\hline 1112.00 & 3.47 & 0.06 & 0.88 & 0.38 \\
\hline 1213.00 & 2.84 & 0.53 & 0.56 & 0.46 \\
\hline 1299.00 & 2.68 & 0.31 & 3.12 & 0.11 \\
\hline 1408.00 & 3.90 & 0.07 & 0.39 \\
\hline
\end{tabular}




\section{Shape}

FWHM LOW Tail

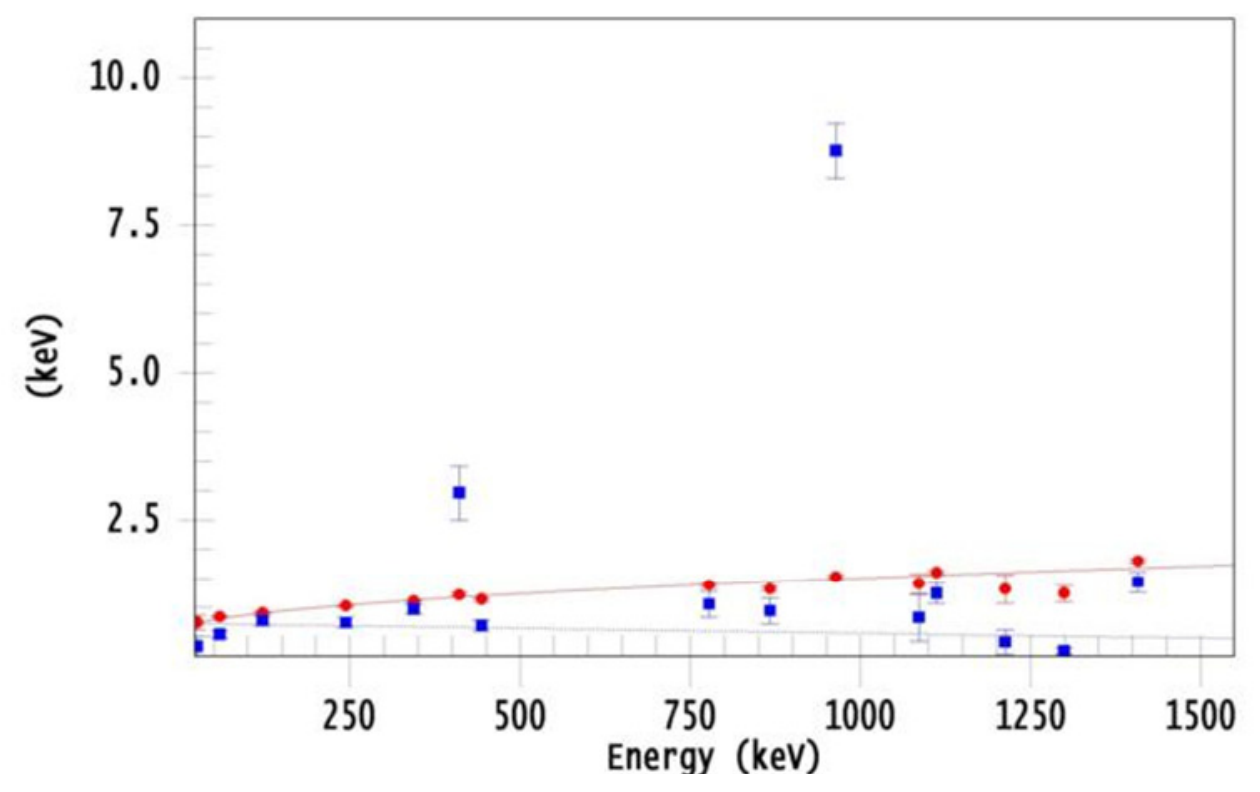


Energy Calibration

Battelle Energy Alliance LLC

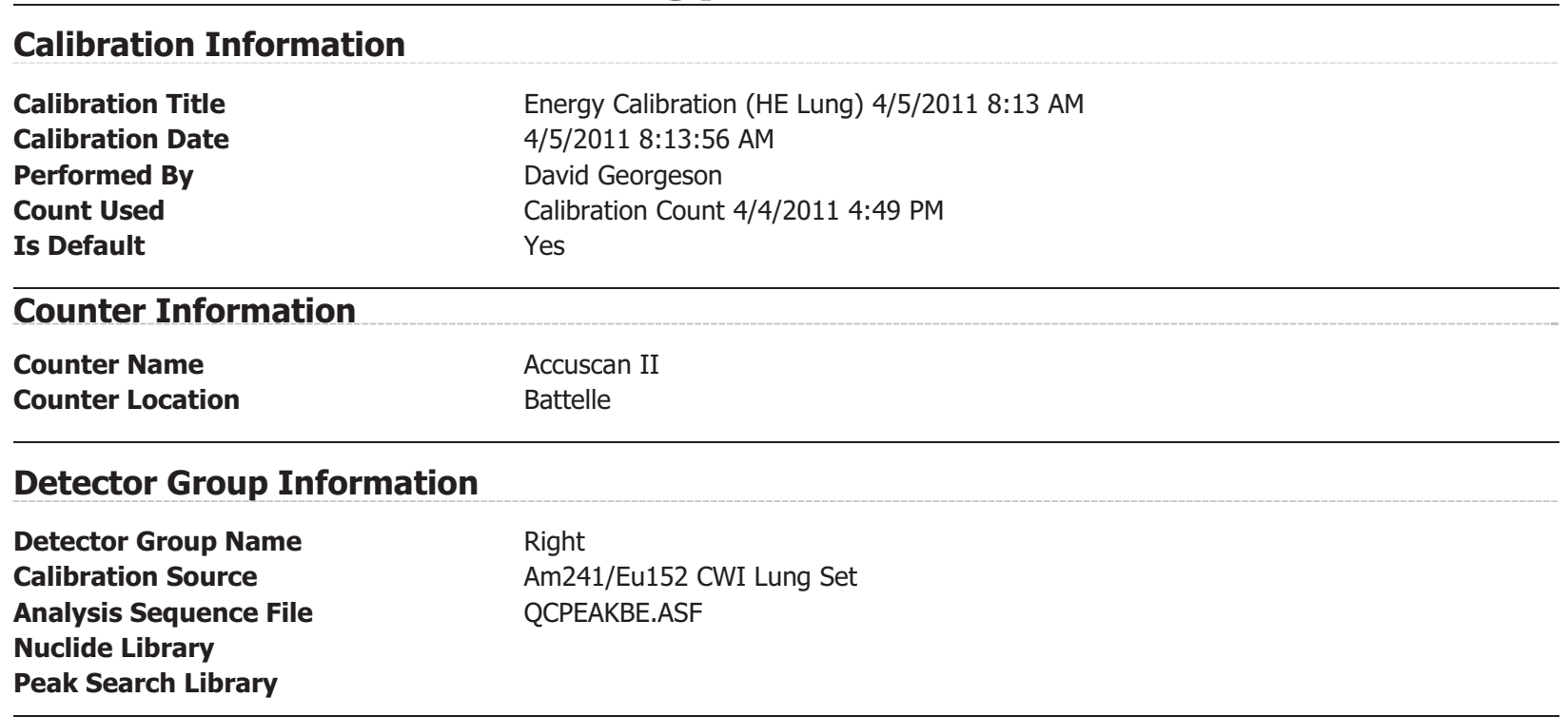

\section{Energy Calibration Coefficients}

$$
\begin{aligned}
\text { Energy } & =-0.244432+0.461855 * \mathrm{ch}+3.89635 \mathrm{e}-08 * \mathrm{ch}^{\wedge} 2 \\
\text { FWHM } & =0.577324+0.0294204 * \mathrm{E}^{\wedge} 1 / 2 \\
\text { LOW TAIL } & =0.726181-0.000167099 * \mathrm{E}
\end{aligned}
$$

\begin{tabular}{|c|c|c|}
\hline \multicolumn{3}{|c|}{ Energy Calibration Results Table } \\
\hline Centroid Channel & Centroid Error & Energy (keV) \\
\hline 57.61 & 0.09 & 26.34 \\
\hline 129.57 & 0.02 & 59.54 \\
\hline 264.23 & 0.01 & 121.78 \\
\hline 530.24 & 0.02 & 244.70 \\
\hline 745.81 & 0.01 & 344.28 \\
\hline 890.50 & 0.03 & 411.10 \\
\hline 961.61 & 0.03 & 443.97 \\
\hline 1686.55 & 0.04 & 778.00 \\
\hline 1878.09 & 0.04 & 867.40 \\
\hline 2087.36 & 0.03 & 964.10 \\
\hline 2350.75 & 0.16 & 1086.00 \\
\hline 2407.67 & 0.03 & 1112.00 \\
\hline 2626.15 & 0.23 & 1213.00 \\
\hline 2813.14 & 0.30 & 1299.00 \\
\hline 3048.21 & 0.04 & 1408.00 \\
\hline
\end{tabular}

Approved by:

Date: 


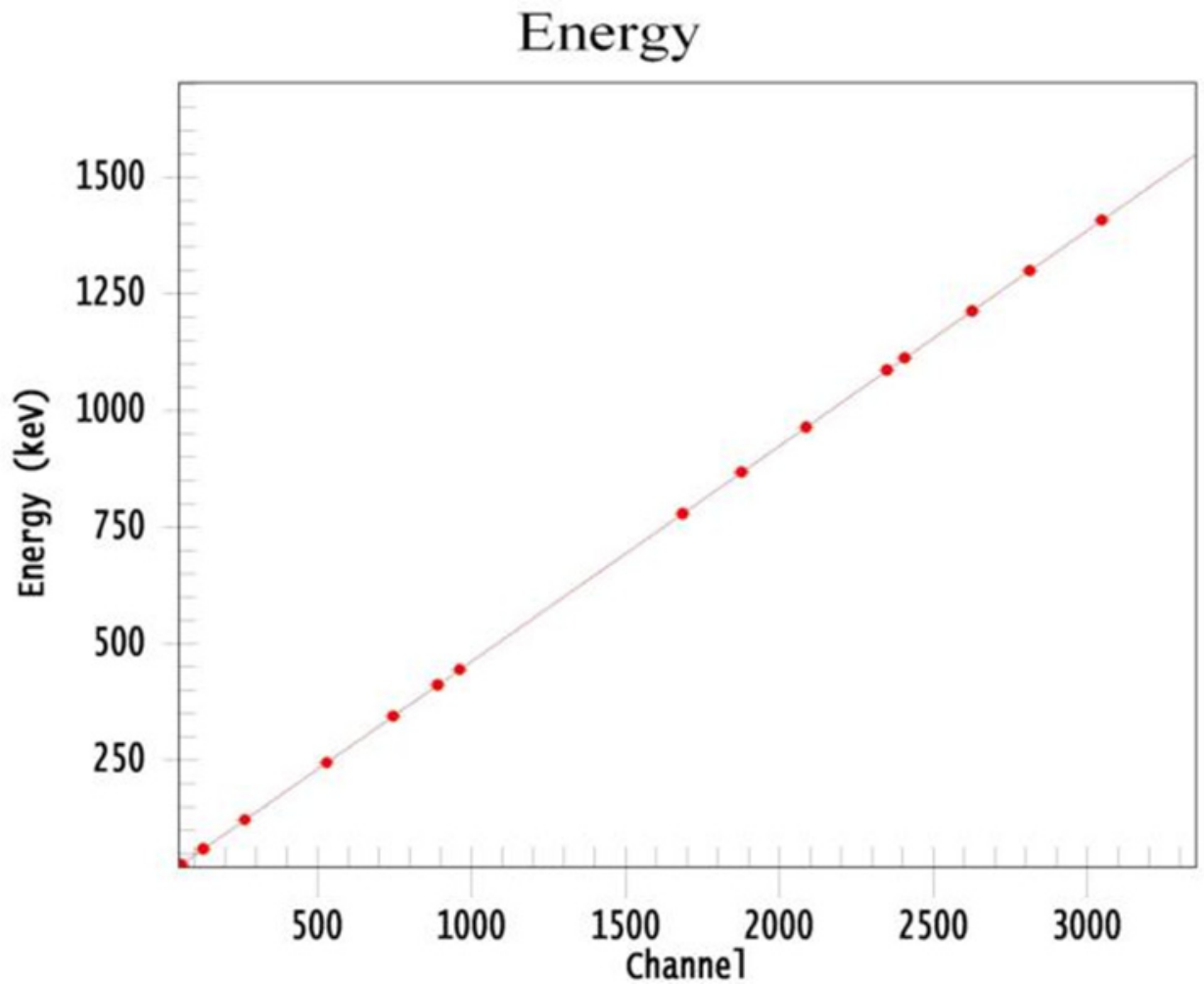

\section{Energy Calibration Results Table}

\begin{tabular}{rrrrr}
\hline Energy $(\mathbf{k e V})$ & FWHM Channels & FWHM Error & TAIL Channels & $\frac{\text { TAIL Error }}{0.37}$ \\
\cline { 2 - 5 } 26.34 & 1.61 & 0.27 & 0.72 & 1.16 \\
\hline 59.54 & 1.82 & 0.03 & 1.67 & 0.12 \\
\hline 121.78 & 1.97 & 0.02 & 1.60 & 0.18 \\
\hline 244.70 & 2.21 & 0.04 & 2.09 & 0.19 \\
\hline 344.28 & 2.39 & 0.02 & 6.42 & 0.18 \\
\hline 411.10 & 2.63 & 0.07 & 1.49 & 1.00 \\
\hline 443.97 & 2.46 & 0.06 & 2.28 & 0.20 \\
\hline 778.00 & 2.99 & 0.08 & 2.04 & 0.48 \\
\hline 867.40 & 2.85 & 0.08 & 18.97 & 0.48 \\
\hline 964.10 & 3.31 & 0.06 & 1.78 & 1.00 \\
\hline 1086.00 & 3.06 & 0.36 & 2.69 & 0.86 \\
\hline 1112.00 & 3.47 & 0.06 & 0.88 & 0.38 \\
\hline 1213.00 & 2.84 & 0.53 & 0.56 & 0.46 \\
\hline 1299.00 & 2.68 & 0.31 & 3.12 & 0.11 \\
\hline 1408.00 & 3.90 & 0.07 & 0.39 \\
\hline
\end{tabular}


Energy Calibration

Battelle Energy Alliance LLC

\section{Calibration Information}

Calibration Title

Calibration Date

Performed By

Count Used

Is Default

\section{Counter Information}

Counter Name

Counter Location
Energy Calibration (HE Lung) 4/5/2011 8:18 AM

4/5/2011 8:19:05 AM

David Georgeson

Calibration Count 4/4/2011 4:49 PM

Yes

\section{Detector Group Information}

\begin{tabular}{ll}
$\begin{array}{l}\text { Detector Group Name } \\
\text { Calibration Source } \\
\text { Analysis Sequence File } \\
\text { Nuclide Library }\end{array}$ & $\begin{array}{l}\text { Summed } \\
\text { Am241/Eu152 CWI Lung Set }\end{array}$ \\
Peak Search Library & Am241/Eu152 CWI Lung Set \\
\hline
\end{tabular}

\section{Energy Calibration Coefficients}

$$
\begin{aligned}
\text { Energy } & =-0.0852164+0.461564 * \mathrm{ch}+1.38308 \mathrm{e}-07 * \mathrm{ch}{ }^{\wedge} 2 \\
\text { FWHM } & =0.790531+0.0308963 * \mathrm{E}^{\wedge} 1 / 2 \\
\text { LOW TAIL }= & 0.590587+0.000758722 * \mathrm{E}
\end{aligned}
$$

\section{Energy Calibration Results Table}

\begin{tabular}{|rrr} 
Centroid Channel & Centroid Error & Energy $(\mathbf{k e V})$ \\
\cline { 2 - 3 } 57.59 & 0.09 & 26.34 \\
\hline 129.53 & 0.03 & 59.54 \\
\hline 264.16 & 0.02 & 121.78 \\
\hline 530.11 & 0.02 & 244.70 \\
\hline 745.63 & 0.02 & 344.28 \\
\hline 890.24 & 0.03 & 411.10 \\
\hline 961.37 & 0.03 & 443.97 \\
\hline 1686.31 & 0.02 & 778.00 \\
\hline 1877.81 & 0.05 & 867.40 \\
\hline 2087.02 & 0.03 & 964.10 \\
\hline 2350.40 & 0.12 & 1086.00 \\
\hline 2407.40 & 0.04 & 1112.00 \\
\hline 2625.58 & 0.14 & 1213.00 \\
\hline 2812.33 & 0.11 & 1299.00 \\
\hline 3047.92 & 0.02 & 1408.00 \\
\hline
\end{tabular}

Approved by:

Date: 


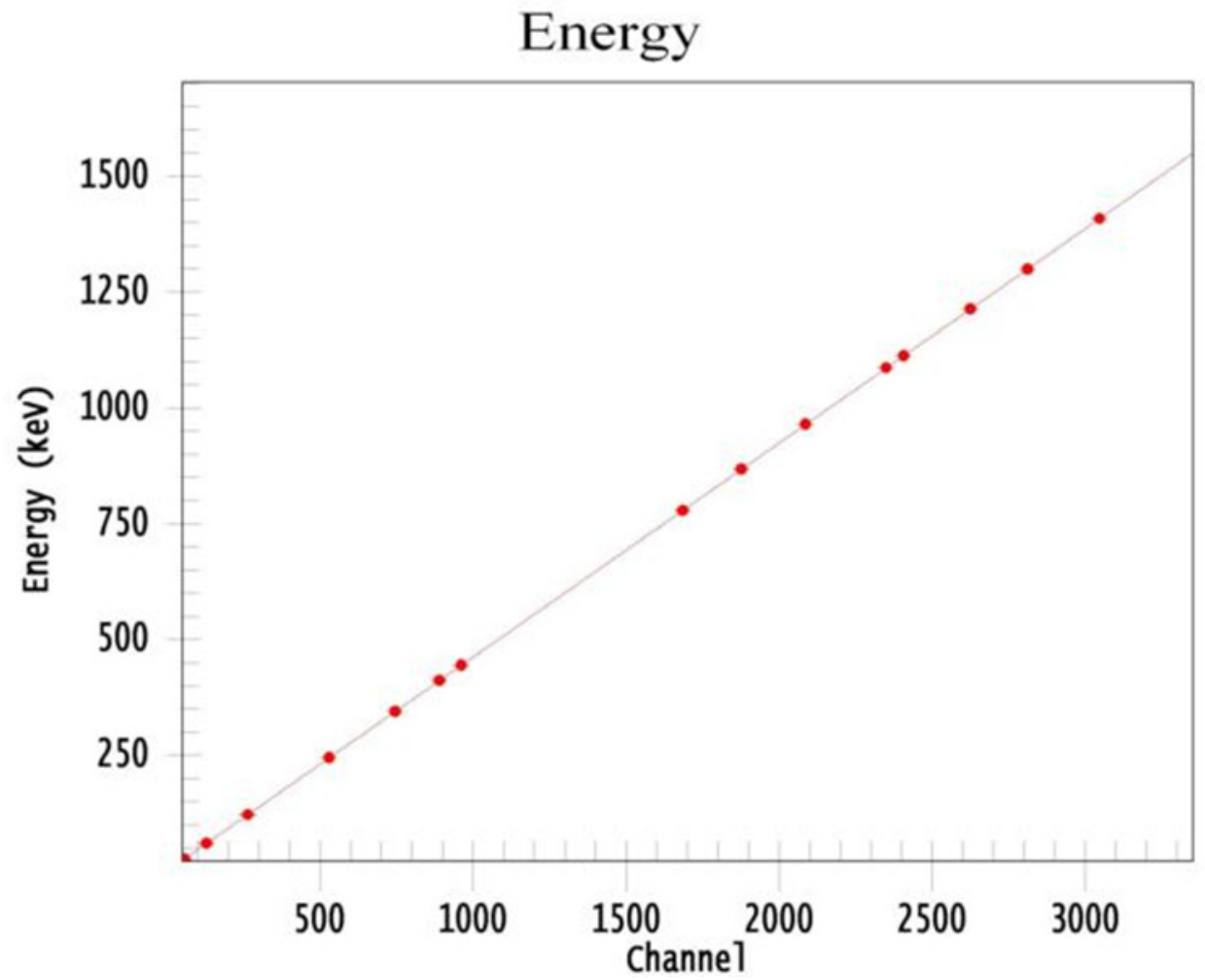

\section{Energy Calibration Results Table}

\begin{tabular}{rrrrrr}
\hline Energy $(\mathbf{k e V})$ & FWHM Channels & FWHM Error & TAIL Channels & $\frac{\text { TAIL Error }}{0.23}$ \\
\hline 26.34 & 1.96 & 0.23 & 0.70 & 0.18 \\
\hline 59.54 & 2.37 & 0.06 & 1.42 & 0.16 \\
\hline 121.78 & 2.46 & 0.05 & 1.52 & 0.19 \\
\hline 244.70 & 2.75 & 0.04 & 1.84 & 0.24 \\
\hline 344.28 & 2.91 & 0.05 & 2.17 & 1.00 \\
\hline 411.10 & 2.99 & 0.06 & 6.12 & 0.22 \\
\hline 443.97 & 3.17 & 0.06 & 1.96 & 0.26 \\
\hline 778.00 & 3.47 & 0.05 & 2.58 & 0.55 \\
\hline 867.40 & 3.55 & 0.11 & 2.45 & 1.00 \\
\hline 964.10 & 3.77 & 0.05 & 5.69 & 1.62 \\
\hline 1086.00 & 3.46 & 0.27 & 2.74 & 0.25 \\
\hline 1112.00 & 3.93 & 0.08 & 2.55 & 2.44 \\
\hline 1213.00 & 4.34 & 0.32 & 3.36 & 6.09 \\
\hline 1299.00 & 3.89 & 0.24 & 3.81 & 0.32 \\
\hline 1408.00 & 4.33 & 0.04 & 3.83 & \\
\hline
\end{tabular}


Appendix D

\section{Efficiency Calibration Documentation}




\section{Appendix D \\ Efficiency Calibration Documentation

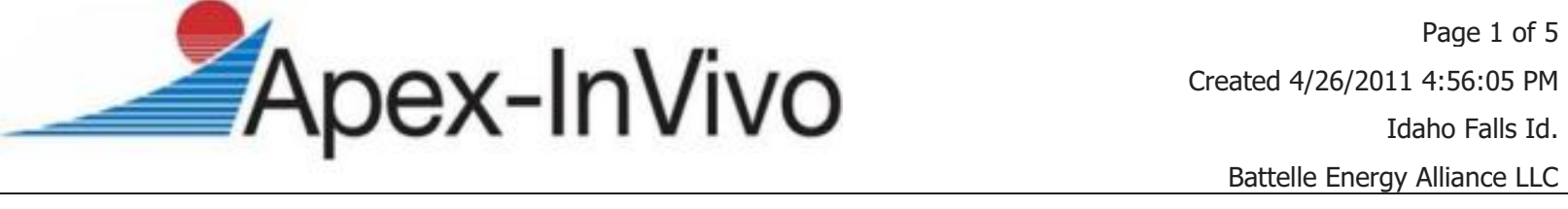 \\ Efficiency Calibration}

\section{Calibration Information}

Calibration Title

Calibration Date

Performed By

Count Used

Approved

Is Default

Is Multi Curve
Eff. Calibration (HE Lung Realistic)4/5/2011 4:05 PM

4/5/2011 4:03:37 PM

David Georgeson

Calibration Count 4/5/2011 2:02 PM

4/7/2011 9:16:28 AM by David Georgeson

Yes

No

\section{Counter Information}

Counter Name

Counter Location

Counter Configuration
Accuscan II

Battelle

Lung - High Energy

\section{Detector Group Information}

Detector Group Name

Calibration Source

Analysis Sequence File

Nuclide Library

Peak Search Library
Summed

Am241/Eu152 CWI Lung Set ROUNPPGE.ASF

Am241/Eu152 CWI Lung Set

Am241/Eu152 CWI Lung Set

Approved by:

Date: 


\section{Efficiency Triplets}

\begin{tabular}{|c|c|c|c|c|c|c|c|c|}
\hline Energy & Efficiency & Error & Dual & Difference & Empirical & Difference & Linear & Difference \\
\hline $2.634 \mathrm{E}+001$ & $1.047 \mathrm{E}-003$ & $1.464 \mathrm{E}-005$ & $1.047 \mathrm{E}-003$ & $0.03 \%$ & $1.047 \mathrm{E}-003$ & $0.04 \%$ & $1.047 \mathrm{E}-003$ & $0.00 \%$ \\
\hline $5.954 \mathrm{E}+001$ & $1.275 \mathrm{E}-003$ & 4.390E-006 & $1.275 \mathrm{E}-003$ & $-0.02 \%$ & $1.275 \mathrm{E}-003$ & $-0.02 \%$ & $1.275 \mathrm{E}-003$ & $0.00 \%$ \\
\hline $1.218 \mathrm{E}+002$ & $1.595 \mathrm{E}-003$ & $1.499 \mathrm{E}-005$ & $1.589 \mathrm{E}-003$ & $0.38 \%$ & $1.588 \mathrm{E}-003$ & $0.39 \%$ & $1.593 \mathrm{E}-003$ & $0.09 \%$ \\
\hline $2.447 \mathrm{E}+002$ & $1.105 \mathrm{E}-003$ & $2.485 \mathrm{E}-005$ & $1.151 \mathrm{E}-003$ & $-4.18 \%$ & $1.151 \mathrm{E}-003$ & $-4.17 \%$ & $1.143 \mathrm{E}-003$ & $-3.41 \%$ \\
\hline $3.443 E+002$ & $8.668 \mathrm{E}-004$ & $8.175 \mathrm{E}-006$ & $8.654 \mathrm{E}-004$ & $0.17 \%$ & 8.653E-004 & $0.17 \%$ & $8.626 \mathrm{E}-004$ & $0.49 \%$ \\
\hline $4.111 \mathrm{E}+002$ & 8.097E-004 & $5.015 \mathrm{E}-005$ & 7.390E-004 & $8.74 \%$ & 7.390E-004 & $8.74 \%$ & 7.424E-004 & $8.32 \%$ \\
\hline $4.440 \mathrm{E}+002$ & 7.315E-004 & $3.472 \mathrm{E}-005$ & $6.903 \mathrm{E}-004$ & $5.63 \%$ & $6.903 \mathrm{E}-004$ & $5.63 \%$ & $6.961 \mathrm{E}-004$ & $4.85 \%$ \\
\hline $7.780 \mathrm{E}+002$ & $4.318 \mathrm{E}-004$ & 8.934E-006 & $4.382 \mathrm{E}-004$ & $-1.48 \%$ & 4.382E-004 & $-1.47 \%$ & 4.446E-004 & $-2.95 \%$ \\
\hline $8.674 \mathrm{E}+002$ & $4.180 \mathrm{E}-004$ & $2.106 \mathrm{E}-005$ & $4.053 \mathrm{E}-004$ & $3.04 \%$ & $4.052 \mathrm{E}-004$ & $3.04 \%$ & $4.091 \mathrm{E}-004$ & $2.12 \%$ \\
\hline $9.641 \mathrm{E}+002$ & $3.794 \mathrm{E}-004$ & 7.380E-006 & 3.762E-004 & $0.84 \%$ & $3.762 \mathrm{E}-004$ & $0.84 \%$ & 3.775E-004 & $0.50 \%$ \\
\hline $1.086 \mathrm{E}+003$ & 3.435E-004 & 7.133E-006 & 3.457E-004 & $-0.64 \%$ & 3.457E-004 & $-0.64 \%$ & 3.447E-004 & $-0.34 \%$ \\
\hline $1.112 \mathrm{E}+003$ & 3.373E-004 & 7.344E-006 & 3.399E-004 & $-0.76 \%$ & 3.399E-004 & $-0.76 \%$ & $3.385 \mathrm{E}-004$ & $-0.36 \%$ \\
\hline $1.408 \mathrm{E}+003$ & $2.830 \mathrm{E}-004$ & 4.676E-006 & 2.821E-004 & $0.30 \%$ & $2.821 \mathrm{E}-004$ & $0.30 \%$ & 2.815E-004 & $0.52 \%$ \\
\hline
\end{tabular}

\section{Dual}

$\ln ($ eff $)=125.58-132.962 * \ln (E)+51.6697 *(\ln (E))^{\wedge} 2-9.71828 *(\ln (E))^{\wedge} 3+0.887749 *(\ln (E))^{\wedge} 4-$ $0.0316986 *(\ln (\mathrm{E}))^{\wedge} 5$

where $\mathrm{E}=$ Energy in keV

\section{Dual}

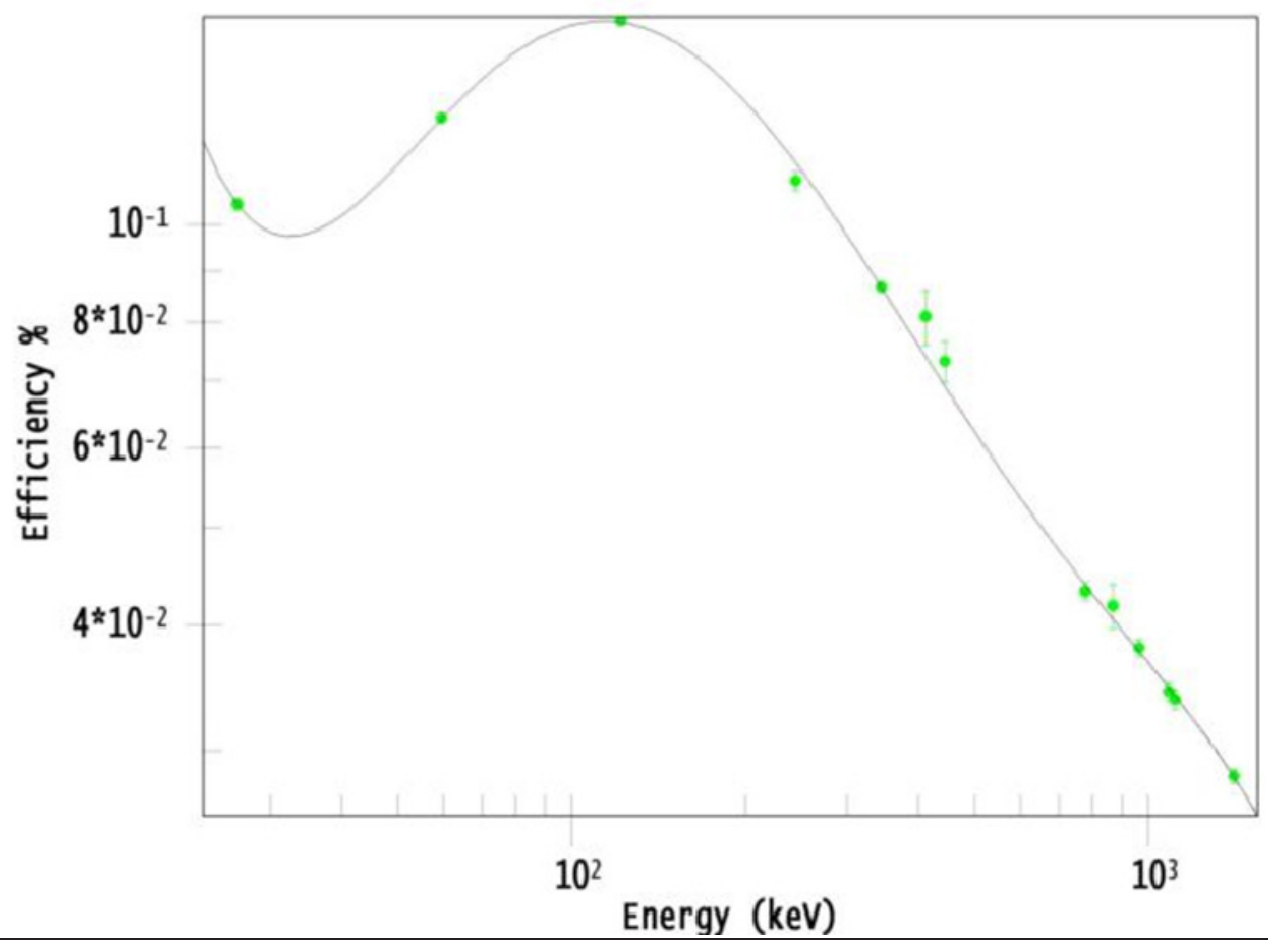




\section{Interpolated}

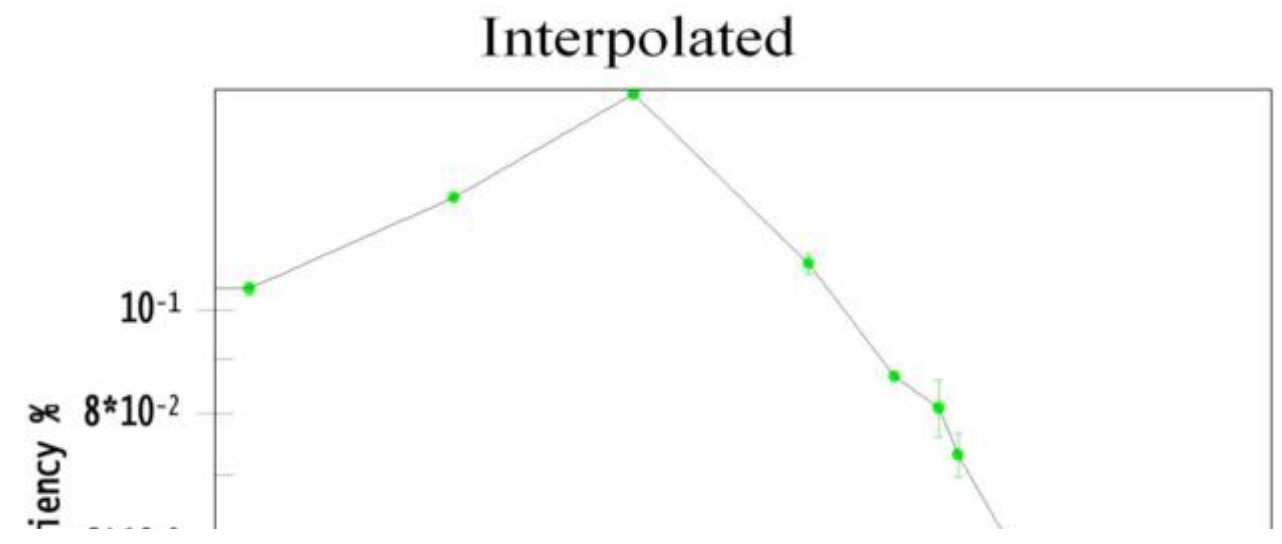

User: David Georgeson

Database: InVivo Server: 127.0.0.1 


\section{Linear}

$\log ($ eff $)=-0.000149837 * E-3.48852+226.571 / E-24386.4 / E^{\wedge} 2+999396 / E^{\wedge} 3-1.32987 e+07 /$ $\mathrm{E}^{\wedge} 4$

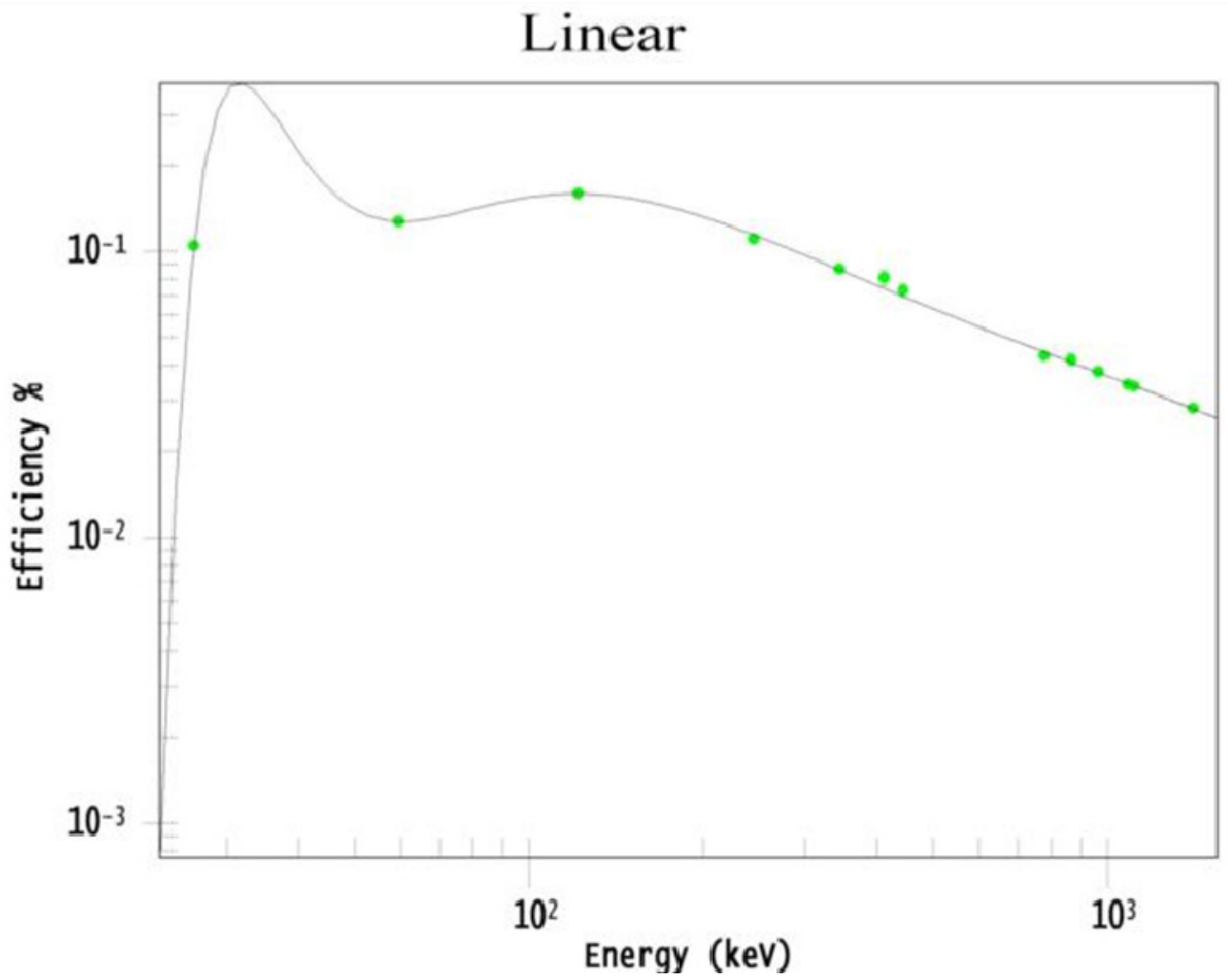




\section{Empirical}

$\ln ($ eff $)=-7.6726+0.751366 * x+0.143931 * x^{\wedge} 2+0.0741517 * x^{\wedge} 3-0.154391 * x^{\wedge} 4+0.0316986 *$ $x^{\wedge} 5$

where $x=\ln (717.17 / E)$

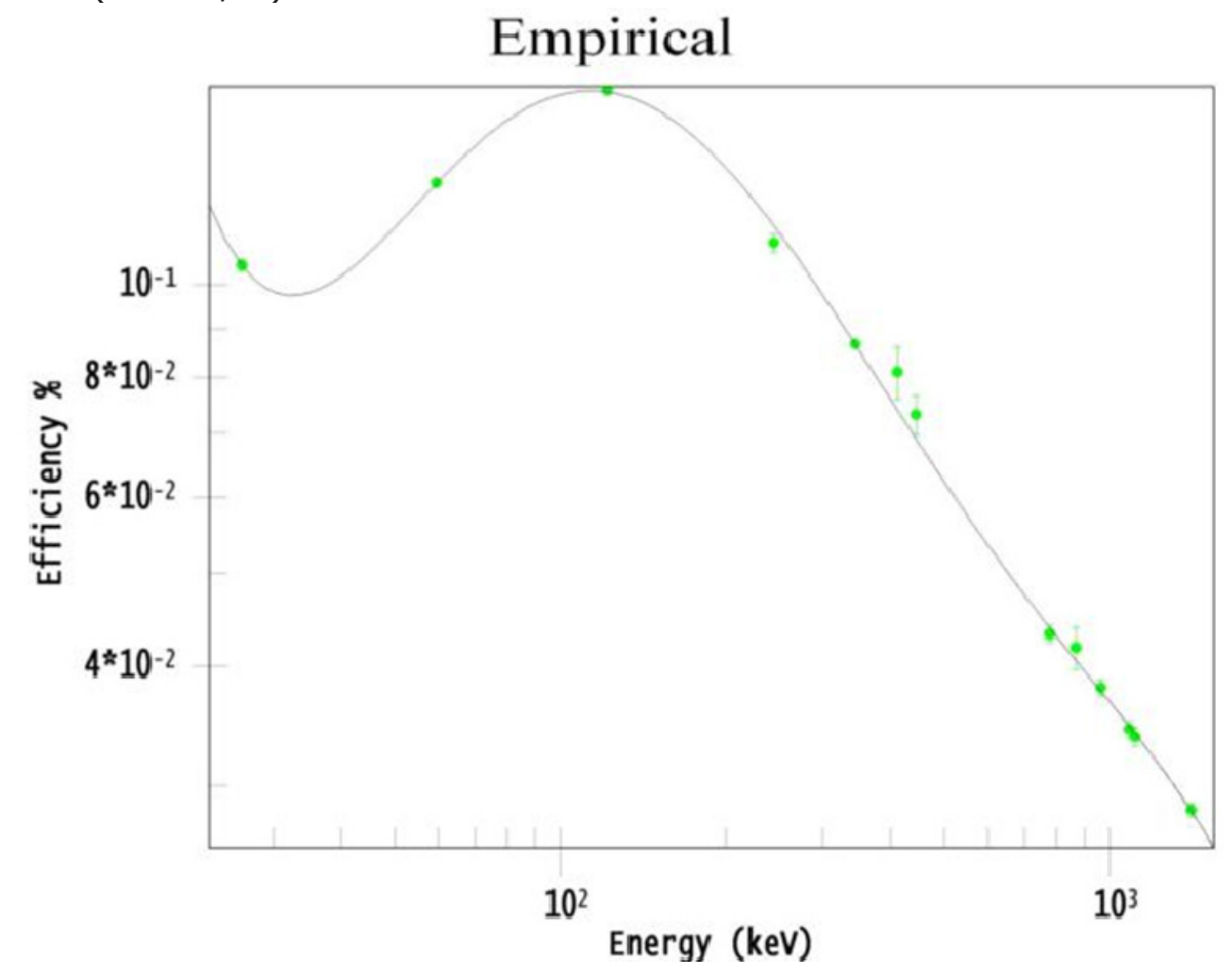




\section{Appendix E}

\section{High Energy Lung Calibration Count Reanalysis}




\title{
Appendix E High Energy Lung Calibration Count Reanalysis Summary
}

\author{
Reanalysis of the Calibration Count \\ Arrangement/Geometry: High Energy Lung \\ Analysis Library: Am241/Eu152 CWI Lung Set.NLB \\ Energy Calibration Time: 4/5/2011 8:18 AM \\ Efficiency Calibration Time: 4/5/2011 4:05 PM \\ Count Type/Live Time: 7226.91 seconds \\ Count Start Times: \\ 4/5/2011 2:02 PM
}

\begin{tabular}{lrccc}
$\begin{array}{l}\text { Nuclide } \\
\begin{array}{l}\text { Energy } \\
(\mathrm{keV})\end{array}\end{array}$ & $\begin{array}{l}\text { Energy } \\
\mathrm{keV}\end{array}$ & $\begin{array}{l}\text { Expected } \\
\text { Activity } \\
(\mathrm{nCi})\end{array}$ & $\begin{array}{c}\text { Count Results } \\
(\mathrm{nCi})\end{array}$ & $\begin{array}{c}\text { Relative } \\
\text { Bias }\end{array}$ \\
\hline Am-241 & 26.44 & 1218.1 & 1242 & 0.02 \\
& 59.69 & 1218.1 & 1256.6 & 0.03 \\
Eu-152 & 121.84 & 253.78 & 256.6 & 0.01 \\
& 244.61 & 253.78 & 245.9 & -0.03 \\
& 344.18 & 253.78 & 257.1 & 0.01 \\
& 410.97 & 253.78 & 265.9 & 0.05 \\
& 443.78 & 253.78 & 267.9 & 0.06 \\
& 778.69 & 253.78 & 250.9 & -0.01 \\
& 867.1 & 253.78 & 263.4 & 0.04 \\
& 963.82 & 253.78 & 254.9 & 0 \\
& 1085.67 & 253.78 & 251.5 & -0.01 \\
& 1111.89 & 253.78 & 248.7 & -0.02 \\
& 1408.06 & 253.78 & 254.4 & 0 \\
& & & Average & 0.011538
\end{tabular}




\section{Analysis Report - Calibration Count 4/5/2011 2:02 PM}

\section{COUNTER INFORMATION}

Counter Name

Counter Location

Configuration Name
Accuscan II

Battelle

Lung - High Energy

\section{COUNT INFORMATION}

\begin{tabular}{ll}
$\begin{array}{l}\text { Count Operation } \\
\text { Comment } \\
\text { Intake Date }\end{array}$ & Calibration Count \\
Acquisition Started & \\
Acquisition Time & $4 / 5 / 20112: 02: 02$ PM \\
Operator Name & 7226.91 \\
Count Status & David Georgeson \\
Primary Review & Not Reviewed \\
Secondary Review & No \\
\hline
\end{tabular}

\section{DETECTOR COUNT RATE REPORT}

\begin{tabular}{|l|r|c|c|c|c|l|}
\hline \multicolumn{1}{|c|}{ Detector Name } & $\begin{array}{c}\text { Count } \\
\text { Rate }\end{array}$ & $\begin{array}{c}\text { Count } \\
\text { Rate }\end{array}$ & Live Time & Analyzed & Analyzed Date & File Name \\
\hline Left & 80.44 & $1-4096$ & 7200.0 & Yes & $4 / 5 / 20112: 01: 56$ PM & b35455fe1401.cnf \\
\hline Right & 83.42 & $1-4096$ & 7200.0 & Yes & $4 / 5 / 20112: 01: 56$ PM & $44 a b f 7001401 . c n f$ \\
\hline Summed & 0.00 & $1-4096$ & 7200.0 & Yes & $4 / 27 / 20119: 25: 00$ AM & $0514 \mathrm{db} 5 \mathrm{c} 1401 . \mathrm{cnf}$ \\
\hline
\end{tabular}

Primary Review by:

Date:

User: David Georgeson
Secondary Review by:

Date: 


\section{DETECTOR GROUP INFORMATION}

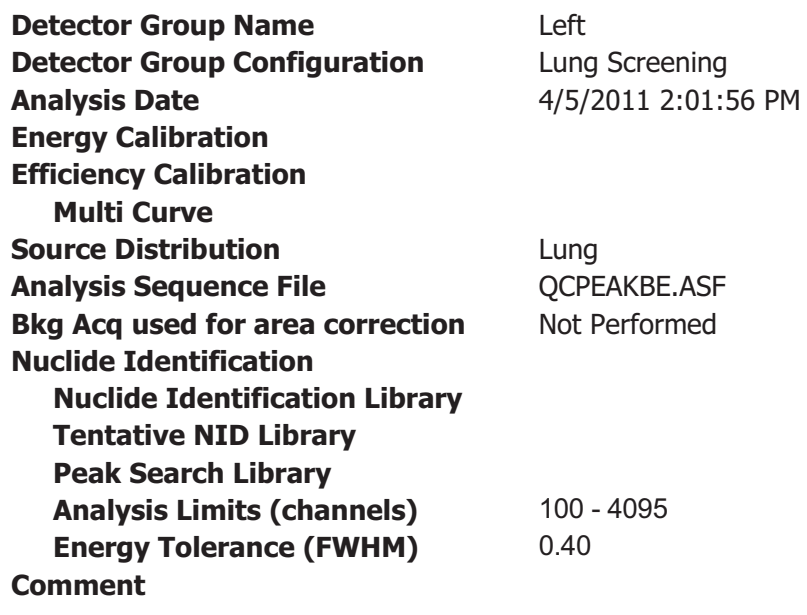

PEAK SEARCH RESULTS FOR Accuscan II < Left>

\begin{tabular}{|c|c|c|c|c|c|c|c|c|c|}
\hline \multicolumn{6}{|c|}{ Peak Analysis } & \multicolumn{4}{|c|}{ Nuclide Information } \\
\hline & No & \begin{tabular}{|l|} 
Peak \\
Centroid
\end{tabular} & \begin{tabular}{|l} 
Energy \\
(keV)
\end{tabular} & \begin{tabular}{|l|} 
Net Peak \\
Area
\end{tabular} & $\begin{array}{l}\text { Continuum } \\
\text { Counts }\end{array}$ & $\begin{array}{l}\text { Nuclide } \\
\text { Name }\end{array}$ & Activity (nCi) & Error (1SD) & \begin{tabular}{|l} 
Yield \\
$(\%)$
\end{tabular} \\
\hline $\mathrm{F}$ & 1 & 108.12 & 49.71 & $2.08 \mathrm{E}+003$ & $2.56 \mathrm{E}+004$ & Unknown & & & 100.00 \\
\hline $\mathrm{F}$ & 2 & 129.50 & 59.59 & $7.31 \mathrm{E}+004$ & $2.32 \mathrm{E}+004$ & Unknown & & & 100.00 \\
\hline $\mathrm{F}$ & 3 & 264.14 & 121.79 & $1.52 \mathrm{E}+004$ & $8.98 \mathrm{E}+003$ & Unknown & & & 100.00 \\
\hline $\mathrm{F}$ & 4 & 530.00 & 244.62 & $2.80 \mathrm{E}+003$ & $2.70 \mathrm{E}+003$ & Unknown & & & 100.00 \\
\hline $\mathrm{F}$ & 5 & 745.65 & 344.24 & $7.70 \mathrm{E}+003$ & $1.14 \mathrm{E}+003$ & Unknown & & & 100.00 \\
\hline $\mathrm{F}$ & 6 & 796.44 & 367.71 & $2.24 \mathrm{E}+002$ & $7.03 E+002$ & Unknown & & & 100.00 \\
\hline $\mathrm{F}$ & 7 & 890.29 & 411.07 & $5.64 \mathrm{E}+002$ & $8.11 \mathrm{E}+002$ & Unknown & & & 100.00 \\
\hline $\mathrm{F}$ & 8 & 961.43 & 443.93 & $7.56 \mathrm{E}+002$ & $6.63 E+002$ & Unknown & & & 100.00 \\
\hline $\mathrm{F}$ & 9 & 1686.45 & 778.90 & $1.91 \mathrm{E}+003$ & $4.52 \mathrm{E}+002$ & Unknown & & & 100.00 \\
\hline $\mathrm{F}$ & 10 & 1877.88 & 867.34 & $5.96 \mathrm{E}+002$ & $3.68 \mathrm{E}+002$ & Unknown & & & 100.00 \\
\hline $\mathrm{F}$ & 11 & 2087.03 & 963.98 & $1.85 \mathrm{E}+003$ & $2.47 \mathrm{E}+002$ & Unknown & & & 100.00 \\
\hline $\mathrm{F}$ & 12 & 2350.58 & 1085.75 & $1.06 \mathrm{E}+003$ & $3.40 \mathrm{E}+002$ & Unknown & & & 100.00 \\
\hline $\mathrm{F}$ & 13 & 2407.37 & 1111.98 & $1.53 E+003$ & $2.86 \mathrm{E}+002$ & Unknown & & & 100.00 \\
\hline $\mathrm{F}$ & 14 & 2625.26 & 1212.66 & $1.38 \mathrm{E}+002$ & $1.40 \mathrm{E}+002$ & Unknown & & & 100.00 \\
\hline $\mathrm{F}$ & 15 & 2812.42 & 1299.13 & $1.88 \mathrm{E}+002$ & $6.15 \mathrm{E}+001$ & Unknown & & & 100.00 \\
\hline $\mathrm{F}$ & 16 & 3047.97 & 1407.97 & $1.94 \mathrm{E}+003$ & $6.77 \mathrm{E}+001$ & Unknown & & & 100.00 \\
\hline $\mathrm{F}$ & 17 & 3162.47 & 1460.87 & $2.69 E+002$ & 7.77E+001 & Unknown & & & 100.00 \\
\hline
\end{tabular}

For unknown peaks we assume yield to be $100 \%$ and no decay correction is performed.

$\mathrm{M}=$ First peak in a multiplet region

$\mathrm{m}=$ Other peak in a multiplet region

$\mathrm{F}=$ Fitted singlet 


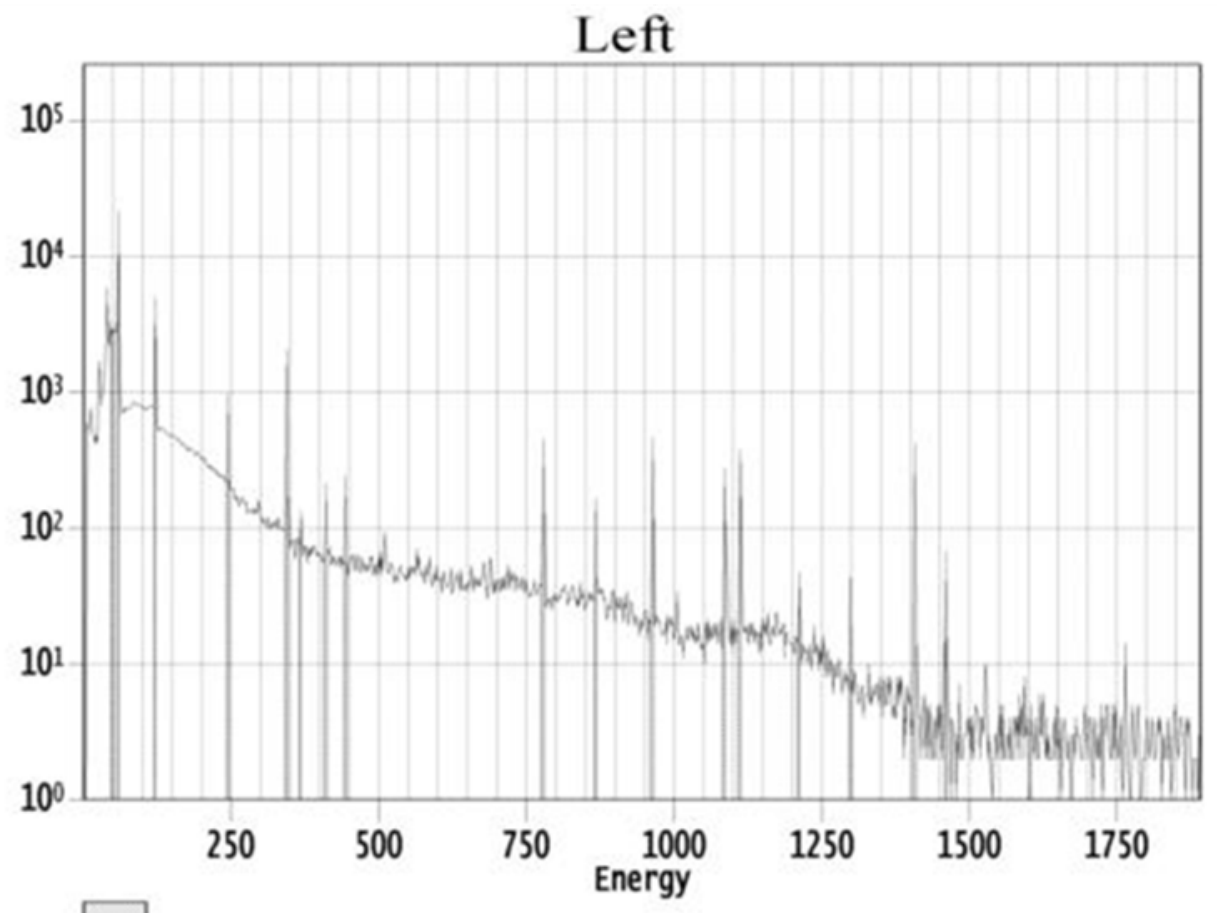

ROI Type: 1

\section{DETECTOR GROUP INFORMATION}

$\begin{array}{ll}\begin{array}{l}\text { Detector Group Name } \\ \text { Detector Group Configuration }\end{array} & \begin{array}{l}\text { Right } \\ \text { Lung Screening } \\ \text { Analysis Date }\end{array} \\ \begin{array}{l}\text { Energy Calibration } \\ \text { Efficiency Calibration }\end{array} \\ \quad \begin{array}{l}\text { Multi Curve } \\ \text { Source Distribution }\end{array} \\ \begin{array}{l}\text { Analysis Sequence File } \\ \text { Bkg Acq used for area correction } \\ \text { Nuclide Identification }\end{array} & \text { Lung } \\ \quad \text { Nuclide Identification Library } & \text { Not Performed } \\ \quad \begin{array}{l}\text { Tentative NID Library } \\ \text { Peak Search Library }\end{array} \\ \quad \begin{array}{l}\text { Analysis Limits (channels) } \\ \text { Energy Tolerance (FWHM) }\end{array} \\ \text { Comment } & 100-4095 \\ \end{array}$

PEAK SEARCH RESULTS FOR Accuscan II <Right>

\begin{tabular}{|c|r|r|r|r|l||l|l|l|l|}
\hline \multicolumn{9}{|c||}{ Peak Analysis } & \multicolumn{4}{|l|}{ Nuclide Information } \\
\hline & No & $\begin{array}{l}\text { Peak } \\
\text { Centroid }\end{array}$ & $\begin{array}{l}\text { Energy } \\
\text { (keV) }\end{array}$ & $\begin{array}{l}\text { Net Peak } \\
\text { Area }\end{array}$ & $\begin{array}{l}\text { Continuum } \\
\text { Counts }\end{array}$ & $\begin{array}{l}\text { Nuclide } \\
\text { Name }\end{array}$ & Activity (nCi) & $\begin{array}{l}\text { Error (1SD) } \\
\text { Yield } \\
\text { (\%) }\end{array}$ \\
\hline F & 1 & 129.55 & 59.59 & $7.46 \mathrm{E}+004$ & $1.46 \mathrm{E}+004$ & Unknown & & 100.00 \\
\hline F & 2 & 264.22 & 121.79 & $1.54 \mathrm{E}+004$ & $5.59 \mathrm{E}+003$ & Unknown & & 100.00 \\
\hline F & 3 & 530.27 & 244.67 & $2.91 \mathrm{E}+003$ & $1.67 \mathrm{E}+003$ & Unknown & & 100.00 \\
\hline
\end{tabular}




\begin{tabular}{|r|r|r|r|r|r||l|l|l|l|}
\hline$F$ & 5 & 796.65 & 367.72 & $2.41 \mathrm{E}+002$ & $5.92 \mathrm{E}+002$ & Unknown & & 100.00 \\
\hline$F$ & 6 & 890.47 & 411.06 & $5.37 \mathrm{E}+002$ & $6.35 \mathrm{E}+002$ & Unknown & & & 100.00 \\
\hline$F$ & 7 & 961.68 & 443.95 & $7.01 \mathrm{E}+002$ & $5.89 \mathrm{E}+002$ & Unknown & & & 100.00 \\
\hline$F$ & 8 & 1686.74 & 778.90 & $1.86 \mathrm{E}+003$ & $3.38 \mathrm{E}+002$ & Unknown & & & 100.00 \\
\hline $\mathrm{F}$ & 9 & 1878.07 & 867.29 & $5.88 \mathrm{E}+002$ & $4.16 \mathrm{E}+002$ & Unknown & & & 100.00 \\
\hline$F$ & 10 & 2087.42 & 964.01 & $1.83 \mathrm{E}+003$ & $2.25 \mathrm{E}+002$ & Unknown & & & 100.00 \\
\hline
\end{tabular}


PEAK SEARCH RESULTS FOR Accuscan II <Right>

\begin{tabular}{|c|c|c|c|c|c|c|c|c|c|}
\hline \multicolumn{6}{|c|}{ Peak Analysis } & \multicolumn{4}{|c|}{ Nuclide Information } \\
\hline & No & \begin{tabular}{|l|} 
Peak \\
Centroid
\end{tabular} & \begin{tabular}{|l|} 
Energy \\
$(\mathrm{keV})$
\end{tabular} & \begin{tabular}{|l|} 
Net Peak \\
Area
\end{tabular} & \begin{tabular}{|l|} 
Continuum \\
Counts
\end{tabular} & $\begin{array}{l}\text { Nuclide } \\
\text { Name }\end{array}$ & Activity (nCi) & Error (1SD) & \begin{tabular}{|l} 
Yield \\
$(\%)$
\end{tabular} \\
\hline M & 11 & 2351.06 & 1085.82 & $1.14 \mathrm{E}+003$ & $1.49 \mathrm{E}+002$ & Unknown & & & 100.00 \\
\hline $\mathrm{m}$ & 12 & 2359.42 & 1089.68 & $2.06 \mathrm{E}+002$ & $1.89 \mathrm{E}+002$ & Unknown & & & 100.00 \\
\hline $\mathrm{F}$ & 13 & 2407.79 & 1112.03 & $1.50 \mathrm{E}+003$ & $2.24 \mathrm{E}+002$ & Unknown & & & 100.00 \\
\hline $\mathrm{F}$ & 14 & 2626.12 & 1212.91 & $1.76 \mathrm{E}+002$ & $1.12 \mathrm{E}+002$ & Unknown & & & 100.00 \\
\hline $\mathrm{F}$ & 15 & 2812.93 & 1299.23 & $1.79 \mathrm{E}+002$ & $6.25 \mathrm{E}+001$ & Unknown & & & 100.00 \\
\hline $\mathrm{F}$ & 16 & 3048.57 & 1408.12 & $1.94 \mathrm{E}+003$ & $8.45 E+001$ & Unknown & & & 100.00 \\
\hline $\mathrm{F}$ & 17 & 3162.79 & 1460.90 & $2.88 \mathrm{E}+002$ & $7.21 \mathrm{E}+001$ & Unknown & & & 100.00 \\
\hline
\end{tabular}

For unknown peaks we assume yield to be $100 \%$ and no decay correction is performed.

$M=$ First peak in a multiplet region

$\mathrm{m}=$ Other peak in a multiplet region

$\mathrm{F}=$ Fitted singlet

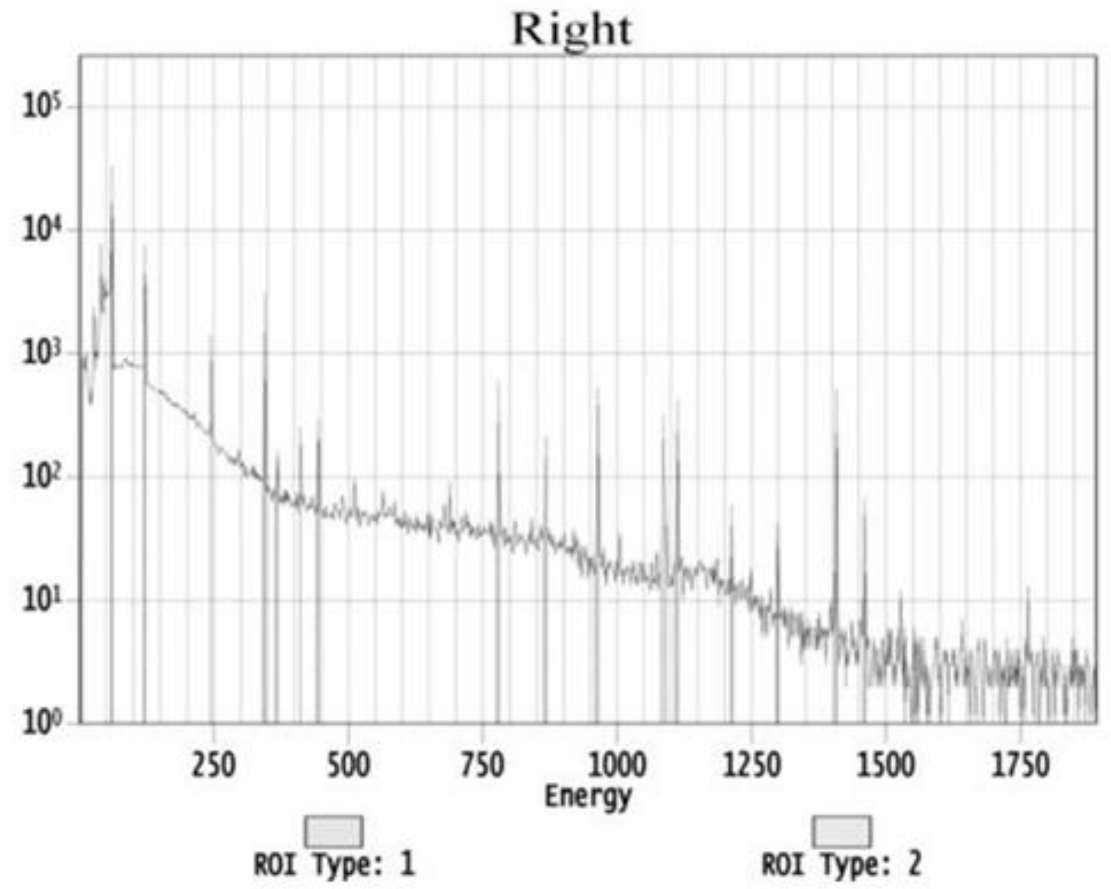

\section{DETECTOR GROUP INFORMATION}

Detector Group Name

Detector Group Configuration

Analysis Date

Energy Calibration

Efficiency Calibration

Multi Curve

Source Distribution

Analysis Sequence File

Bkg Acq used for area correction
Summed

Lung Screening

4/27/2011 9:25:00 AM

Energy Calibration (HE Lung) 4/5/2011 8:18 AM

Eff. Calibration (HE Lung Realistic)4/5/2011 4:05 PM (Dual)

No

Lung

ROUNPPGE.ASF

Not Performed 
Nuclide Identification

Nuclide Identification Library

Am241/Eu152 CWI Lung Set

Tentative NID Library

Peak Search Library

Am241/Eu152 CWI Lung Set

Am241/Eu152 CWI Lung Set

Analysis Limits (channels) $\quad 40-4095$

Energy Tolerance (FWHM)

1.20

Comment

User: David Georgeson

Database: InVivo Server: 127.0.0.1 
PEAK SEARCH RESULTS FOR Accuscan II <Summed>

\begin{tabular}{|c|c|c|c|c|c|c|c|c|c|}
\hline \multicolumn{6}{|c|}{ Peak Analysis } & \multicolumn{4}{|c|}{ Nuclide Information } \\
\hline & No & \begin{tabular}{|l} 
Peak \\
Centroid
\end{tabular} & \begin{tabular}{|l}
$\begin{array}{l}\text { Energy } \\
(\mathrm{keV})\end{array}$ \\
\end{tabular} & \begin{tabular}{|l} 
Net Peak \\
Area
\end{tabular} & $\begin{array}{l}\text { Continuum } \\
\text { Counts }\end{array}$ & $\begin{array}{l}\text { Nuclide } \\
\text { Name }\end{array}$ & Activity (nCi) & Error (1SD) & $\begin{array}{l}\text { Yield } \\
(\%)\end{array}$ \\
\hline & 1 & 45.85 & 21.08 & $5.71 \mathrm{E}+002$ & $6.96 \mathrm{E}+003$ & Unknown & $1.527294 \mathrm{E}+$ & & 100.00 \\
\hline M & 2 & 57.47 & 26.44 & $8.29 E+003$ & $7.74 \mathrm{E}+003$ & Am-241 & $1.241986 \mathrm{E}+$ & $1.99 \%$ & 2.40 \\
\hline $\mathrm{m}$ & 3 & 65.33 & 30.07 & $1.75 \mathrm{E}+003$ & $1.13 \mathrm{E}+004$ & Unknown & $6.689168 \mathrm{E}+$ & & 100.00 \\
\hline M & 4 & 79.65 & 36.68 & $4.52 \mathrm{E}+003$ & $2.68 \mathrm{E}+004$ & Unknown & $1.713211 \mathrm{E}+$ & & 100.00 \\
\hline $\mathrm{m}$ & 5 & 86.83 & 39.99 & $3.75 E+004$ & $4.27 \mathrm{E}+004$ & Unknown & $1.378703 \mathrm{E}+$ & & 100.00 \\
\hline M & 6 & 98.91 & 45.57 & $7.63 E+003$ & $3.92 \mathrm{E}+004$ & Unknown & 2.631127E+ & & 100.00 \\
\hline m & 7 & 107.47 & 49.52 & $3.31 \mathrm{E}+003$ & $6.13 \mathrm{E}+004$ & Unknown & $1.089826 \mathrm{E}+$ & & 100.00 \\
\hline & 8 & 129.51 & 59.69 & $1.48 \mathrm{E}+005$ & $3.44 \mathrm{E}+004$ & $A m-241$ & $1.215659 \mathrm{E}+$ & $0.52 \%$ & 35.70 \\
\hline & 9 & 264.14 & 121.84 & $3.08 \mathrm{E}+004$ & $1.37 \mathrm{E}+004$ & EU-152 & $2.565976 \mathrm{E}+$ & $1.45 \%$ & 28.40 \\
\hline & 10 & 530.07 & 244.61 & $5.65 E+003$ & $3.79 E+003$ & EU-152 & $2.458505 \mathrm{E}+$ & $3.11 \%$ & 7.49 \\
\hline & 11 & 745.70 & 344.18 & $1.58 \mathrm{E}+004$ & $1.74 \mathrm{E}+003$ & EU-152 & $2.570705 \mathrm{E}+$ & $1.38 \%$ & 26.60 \\
\hline & 12 & 796.56 & 367.67 & $3.74 \mathrm{E}+002$ & $1.42 \mathrm{E}+003$ & Unknown & $1.718182 \mathrm{E}+$ & & 100.00 \\
\hline & 13 & 890.33 & 410.97 & $1.15 \mathrm{E}+003$ & $1.44 \mathrm{E}+003$ & EU-152 & $2.648766 \mathrm{E}+$ & $7.47 \%$ & 2.21 \\
\hline & 14 & 961.39 & 443.78 & $1.53 \mathrm{E}+003$ & $1.20 \mathrm{E}+003$ & EU-152 & $2.678836 \mathrm{E}+$ & $5.39 \%$ & 3.11 \\
\hline & 15 & 1221.52 & 563.93 & $3.18 \mathrm{E}+002$ & $1.11 \mathrm{E}+003$ & Unknown & $2.123933 \mathrm{E}+$ & & 100.00 \\
\hline & 16 & 1469.08 & 678.29 & $2.18 \mathrm{E}+002$ & $6.50 E+002$ & Unknown & 1.685309E+ & & 100.00 \\
\hline & 17 & 1491.10 & 688.46 & $3.17 \mathrm{E}+002$ & $9.26 \mathrm{E}+002$ & Unknown & $2.477440 \mathrm{E}+$ & & 100.00 \\
\hline & 18 & 1557.36 & 719.07 & $1.08 \mathrm{E}+002$ & $6.56 \mathrm{E}+002$ & Unknown & 8.741767E- & & 100.00 \\
\hline & 19 & 1686.41 & 778.69 & $3.81 \mathrm{E}+003$ & $8.08 \mathrm{E}+002$ & EU-152 & $2.509298 \mathrm{E}+$ & $2.56 \%$ & 13.00 \\
\hline & 20 & 1822.56 & 841.60 & $1.20 \mathrm{E}+002$ & $5.68 \mathrm{E}+002$ & Unknown & $1.091253 \mathrm{E}+$ & & 100.00 \\
\hline & 21 & 1877.75 & 867.10 & $1.18 \mathrm{E}+003$ & $8.03 E+002$ & EU-152 & $2.633928 \mathrm{E}+$ & $6.08 \%$ & 4.16 \\
\hline & 22 & 1990.44 & 919.18 & $9.74 \mathrm{E}+001$ & $3.74 \mathrm{E}+002$ & Unknown & 9.402959E- & & 100.00 \\
\hline & 23 & 2087.04 & 963.82 & $3.70 \mathrm{E}+003$ & $5.12 \mathrm{E}+002$ & EU-152 & $2.548510 \mathrm{E}+$ & $2.40 \%$ & 14.50 \\
\hline & 24 & 2176.36 & 1005.10 & $1.92 \mathrm{E}+002$ & $4.42 \mathrm{E}+002$ & Unknown & $1.978180 \mathrm{E}+$ & & 100.00 \\
\hline M & 25 & 2350.69 & 1085.67 & $2.36 \mathrm{E}+003$ & $3.19 \mathrm{E}+002$ & EU-152 & 2.515171E+ & $2.31 \%$ & 10.20 \\
\hline $\mathrm{m}$ & 26 & 2358.89 & 1089.46 & $4.06 \mathrm{E}+002$ & $3.42 \mathrm{E}+002$ & Unknown & 4.418480E+ & & 100.00 \\
\hline & 27 & 2407.42 & 1111.89 & $3.05 E+003$ & $5.11 \mathrm{E}+002$ & EU-152 & $2.486969 \mathrm{E}+$ & $2.64 \%$ & 13.55 \\
\hline & 28 & 2625.59 & 1212.75 & $3.02 \mathrm{E}+002$ & $2.96 \mathrm{E}+002$ & Unknown & $3.553563 \mathrm{E}+$ & & 100.00 \\
\hline & 29 & 2812.20 & 1299.02 & $3.49 \mathrm{E}+002$ & $1.77 \mathrm{E}+002$ & Unknown & 4.340987E+ & & 100.00 \\
\hline & 30 & 3048.02 & 1408.06 & $3.99 \mathrm{E}+003$ & $1.17 \mathrm{E}+002$ & EU-152 & $2.542381 \mathrm{E}+$ & $2.33 \%$ & 20.90 \\
\hline & 31 & 3162.42 & 1460.95 & $6.27 \mathrm{E}+002$ & $1.41 \mathrm{E}+002$ & Unknown & $8.631526 E+$ & & 100.00 \\
\hline & 32 & 3308.00 & 1528.28 & $3.68 \mathrm{E}+001$ & $4.92 \mathrm{E}+001$ & Unknown & $5.281573 \mathrm{E}-$ & & 100.00 \\
\hline & 33 & 3820.02 & 1765.11 & $7.85 E+001$ & $4.85 E+001$ & Unknown & $1.319411 \mathrm{E}+$ & & 100.00 \\
\hline
\end{tabular}

For unknown peaks we assume yield to be $100 \%$ and no decay correction is performed.

$M=$ First peak in a multiplet region

$\mathrm{m}=$ Other peak in a multiplet region

$\mathrm{F}=$ Fitted singlet 
NUCLIDE RESULTS FOR Accuscan II <Summed>

\begin{tabular}{|l|l|l|l|r|r|r|r|}
\hline & $\begin{array}{l}\text { Nuclide } \\
\text { Name }\end{array}$ & $\begin{array}{l}\text { Id } \\
\text { Confidence }\end{array}$ & $\begin{array}{l}\text { Wt Mean } \\
\text { Activity (nCi) }\end{array}$ & Error (1SD) & Action Level 1 & Action Level 2 & MDA (nCi) \\
\hline & EU-152 & 0.999 & $2.545804 \mathrm{E}+00$ & $0.70 \%$ & \multirow{2}{*}{ Not Performed } & Not Performed & $3.770 \mathrm{E}+000$ \\
\hline & Am-241 & 0.999 & $1.217271 \mathrm{E}+00$ & $0.50 \%$ & & & $8.264 \mathrm{E}+000$ \\
\hline
\end{tabular}


Analysis Report - Calibration Count 4/5/2011 2:02 PM

$?=$ Nuclide is part of an undetermined solution

$\mathrm{X}=$ Nuclide rejected by the interference analysis

@ = Nuclide contains energy lines not used in Weighted Mean Activity

! = Nuclide was corrected for parent/daughter

NUCLIDE MDA RESULTS FOR Accuscan II <Summed>

\begin{tabular}{|c|c|c|c|c|c|c|}
\hline & $\begin{array}{l}\text { Nuclide } \\
\text { Name }\end{array}$ & Energy (keV) & Yield (\%) & $\begin{array}{l}\text { Line MDA } \\
\text { (nCi) }\end{array}$ & $\begin{array}{l}\text { Nuclide MDA } \\
\text { (nCi) }\end{array}$ & Activity (nCi) \\
\hline+ & \multirow[t]{11}{*}{ EU-152 } & 121.78 * & 28.40 & $5.7831 \mathrm{E}+000$ & \multirow[t]{11}{*}{$3.7696 \mathrm{E}+000$} & $2.5660 \mathrm{E}+002$ \\
\hline+ & & 244.70 * & 7.49 & $1.5430 \mathrm{E}+001$ & & $2.4585 \mathrm{E}+002$ \\
\hline+ & & 344.28 * & 26.60 & $4.3428 \mathrm{E}+000$ & & $2.5707 \mathrm{E}+002$ \\
\hline+ & & 411.10 * & 2.21 & 4.1981E+001 & & $2.6488 \mathrm{E}+002$ \\
\hline+ & & 443.97 * & 3.11 & $2.9096 \mathrm{E}+001$ & & $2.6788 \mathrm{E}+002$ \\
\hline+ & & 778.90 * & 13.00 & $9.5153 E+000$ & & $2.5093 \mathrm{E}+002$ \\
\hline+ & & 867.40 * & 4.16 & $3.2034 \mathrm{E}+001$ & & $2.6339 \mathrm{E}+002$ \\
\hline+ & & 964.13 * & 14.50 & $8.1094 \mathrm{E}+000$ & & $2.5485 \mathrm{E}+002$ \\
\hline+ & & 1086.00 * & 10.20 & $6.2626 \mathrm{E}+000$ & & $2.5152 \mathrm{E}+002$ \\
\hline+ & & 1112.12 * & 13.55 & $9.6235 \mathrm{E}+000$ & & $2.4870 \mathrm{E}+002$ \\
\hline+ & & 1408.01 * & 20.90 & $3.7696 \mathrm{E}+000$ & & $2.5424 \mathrm{E}+002$ \\
\hline+ & \multirow[t]{2}{*}{ Am-241 } & 26.30 * & 2.40 & $4.3428 \mathrm{E}+001$ & \multirow[t]{2}{*}{$8.2644 \mathrm{E}+000$} & $1.2420 \mathrm{E}+003$ \\
\hline+ & & 59.54 * & 35.70 & $8.2644 \mathrm{E}+000$ & & 1.2157E+003 \\
\hline
\end{tabular}

$+=$ Nuclide Identified during the nuclide identification

$*$ = Energy Line found in the spectrum

\section{Summed}

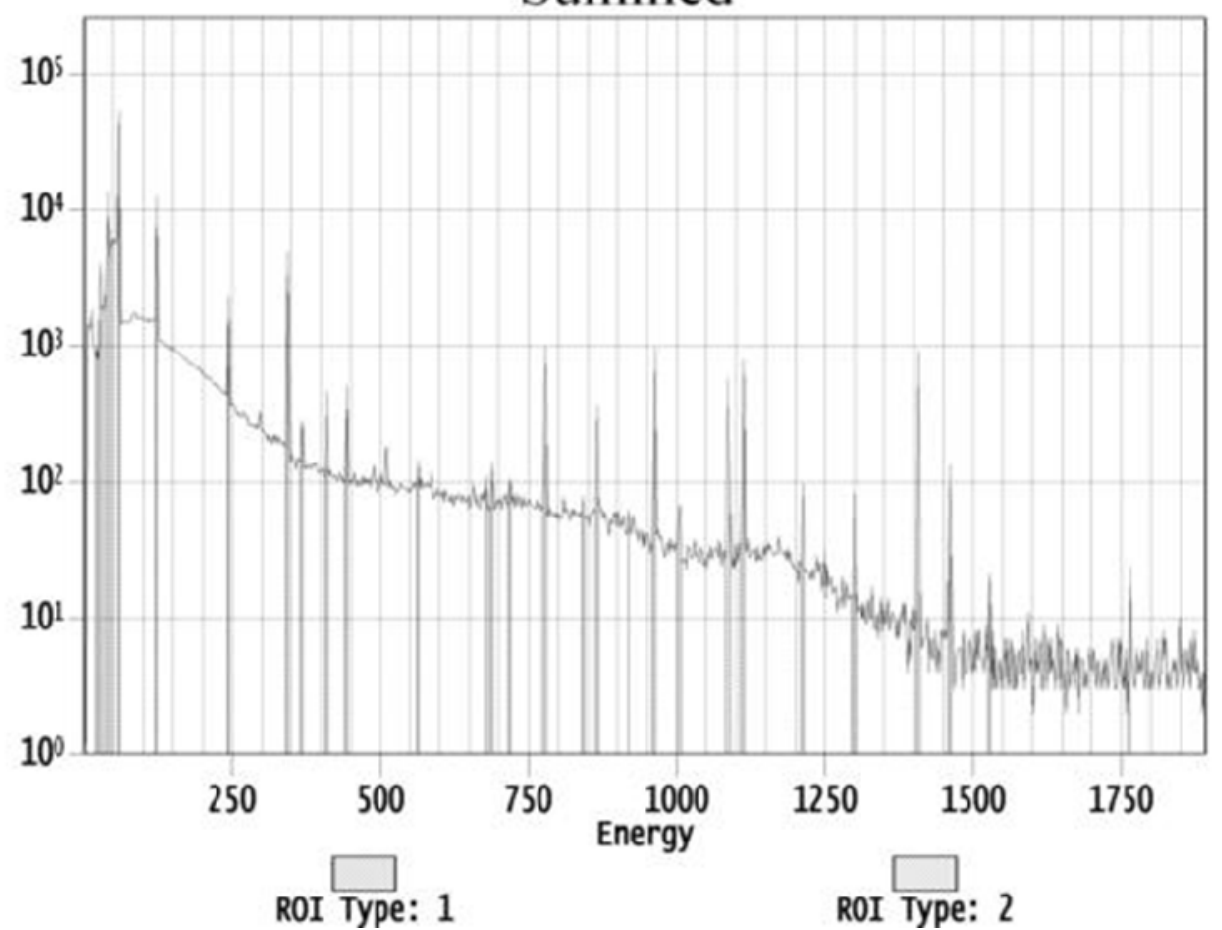


Appendix F

\section{Accuscan II High Energy Lung Calibration Verification}




\section{Appendix $F$ Accuscan-li High Energy Lung Calibration Verification}

Apex-InVivo

Page 1 of 2 Created 4/26/2011 4:35:40 PM Idaho Falls Id.

\section{Efficiency Verification}

\section{Calibration Information}

$\begin{array}{ll}\text { Calibration Title } & \text { Eff. Calibration (HE Lung Realistic)4/5/2011 4:05 PM } \\ \text { Calibration Date } & \text { 4/5/2011 4:03:37 PM } \\ \text { Operator Name } & \text { David Georgeson } \\ \text { Detector Group } & \text { Summed } \\ \text { Detector Group } & \text { Lung Screening } \\ \text { Source Distribution } & \text { Lung }\end{array}$

Counter Information

$\begin{array}{ll}\text { Counter Name } & \text { Accuscan II } \\ \text { Counter Location } & \text { Battelle } \\ \text { Counter } & \text { Lung - High Energy }\end{array}$

\section{Count List}

$\begin{array}{lll}\frac{\text { Num }}{1} & \begin{array}{ll}\text { Date } \\ \text { 4/6/2011 }\end{array} & \text { Name } \\ 2 & 4 / 6 / 2011 & \text { Verification Count 4/6/2011 11:57 AM }(1 / 5) \\ 3 & 4 / 6 / 2011 & \text { Verification Count 4/6/2011 11:57 AM }(2 / 5) \\ 4 & 4 / 6 / 2011 & \text { Verification Count 4/6/2011 11:57 AM }(3 / 5) \\ 5 & 4 / 6 / 2011 & \text { Verification Count 4/6/2011 11:57 AM (4/5) }\end{array}$




\section{Source Certificate Am241/Eu152 CWI Lung Set}

\begin{tabular}{|c|c|c|c|c|c|c|c|c|c|c|}
\hline Nuclide & Energy & $\begin{array}{c}\text { Certificate } \\
\text { Activity } \\
(\mathrm{g} / \mathrm{s})\end{array}$ & Half Life & Count 1 & Count 2 & Count 3 & Count 4 & Count 5 & $\begin{array}{c}\text { Relative } \\
\text { Bias } \\
(\%) \\
\end{array}$ & \begin{tabular}{|c} 
Relative \\
Precision \\
$(\%)$
\end{tabular} \\
\hline \multirow{2}{*}{ Am-241 } & \multirow{2}{*}{26.34} & \multirow{2}{*}{1110.00} & \multirow{2}{*}{$432.19 \mathrm{y}$} & 1016.09 & 1145.33 & 1307.94 & 1073.65 & 1154.98 & \multirow{2}{*}{$5.14 \%$} & \multirow{2}{*}{$9.06 \%$} \\
\hline & & & & 1083.84 & 1083.84 & 1083.84 & 1083.84 & 1083.84 & & \\
\hline \multirow{2}{*}{ Am-241 } & \multirow{2}{*}{59.54} & \multirow{2}{*}{16500.00} & \multirow{2}{*}{$432.19 \mathrm{y}$} & 16413.18 & 16023.05 & 16366.68 & 16520.87 & 16322.24 & \multirow{2}{*}{$1.35 \%$} & \multirow{2}{*}{$1.03 \%$} \\
\hline & & & & 16111.21 & 16111.21 & 16111.21 & 16111.20 & 16111.20 & & \\
\hline \multirow{2}{*}{ EU-152 } & \multirow{2}{*}{121.83} & \multirow{2}{*}{5710.00} & \multirow{2}{*}{$4946.22 \mathrm{~d}$} & 2641.94 & 2646.89 & 2518.99 & 2837.34 & 2652.13 & \multirow{2}{*}{$-0.31 \%$} & \multirow{2}{*}{$3.82 \%$} \\
\hline & & & & 2667.67 & 2667.67 & 2667.66 & 2667.66 & 2667.65 & & \\
\hline \multirow{2}{*}{ EU-152 } & \multirow{2}{*}{244.70} & \multirow{2}{*}{1510.00} & \multirow{2}{*}{$4946.22 \mathrm{~d}$} & 836.12 & 744.21 & 679.54 & 695.02 & 608.73 & \multirow{2}{*}{$1.03 \%$} & \multirow{2}{*}{$10.69 \%$} \\
\hline & & & & 705.46 & 705.46 & 705.46 & 705.46 & 705.46 & & \\
\hline \multirow{2}{*}{ EU-152 } & \multirow{2}{*}{344.28} & \multirow{2}{*}{5350.00} & \multirow{2}{*}{$4946.22 \mathrm{~d}$} & 2460.29 & 2579.14 & 2169.32 & 2454.12 & 2378.46 & \multirow{2}{*}{$-3.65 \%$} & \multirow{2}{*}{$5.43 \%$} \\
\hline & & & & 2499.48 & 2499.48 & 2499.48 & 2499.47 & 2499.47 & & \\
\hline FIl-152 & 41110 & $444 \cap 0$ & 494623 & 151.16 & 268.12 & 265.65 & 168.20 & 172.47 & $-112 \%$ & $2456 \%$ \\
\hline$E U-152$ & 411.10 & 444.00 & $4946.22 \mathrm{~d}$ & 207.43 & 207.43 & 207.43 & 207.43 & 207.43 & $-1.12 \%$ & $24.56 \%$ \\
\hline FI-152 & 44397 & 62500 & 4946 & 232.98 & 339.83 & 283.14 & 364.50 & 305.83 & $454 \%$ & $1563 \%$ \\
\hline EU-152 & 443.97 & 625.00 & $4946.22 d$ & 292.00 & 292.00 & 291.99 & 291.99 & 291.99 & $4.54 \%$ & $15.63 \%$ \\
\hline Fl-152 & 77800 & 261000 & d 2046 & 1389.23 & 998.63 & 1182.95 & 1345.13 & 1284.97 & $171 \%$ & $1141 \%$ \\
\hline$E U-152$ & $1 / 8.00$ & $\angle 610.00$ & $4946.22 \mathrm{a}$ & 1219.37 & 1219.37 & 1219.37 & 1219.37 & 1219.37 & $1.11 \%$ & $11.41 \%$ \\
\hline Fll-152 & 86740 & 83600 & d 2046 & 371.09 & 253.38 & 412.66 & 428.77 & 296.17 & - & $1728 \%$ \\
\hline EU-152 & $86 / .40$ & 836.00 & $4946.22 \mathrm{~d}$ & 390.57 & 390.57 & 390.57 & 390.57 & 390.57 & $-9 . / / \%$ & $1 / .28 \%$ \\
\hline FII-152 & 96410 & 291000 & 494620 & 1516.45 & 1431.85 & 1376.08 & 1396.82 & 1305.95 & $330 \%$ & $500 \%$ \\
\hline$[0-132$ & 904.10 & 2910.00 & $4940.22 \mathrm{U}$ & 1359.53 & 1359.53 & 1359.53 & 1359.52 & 1359.52 & $3.30 \%$ & $3.00 \%$ \\
\hline FI-152 & 108600 & חم ח 205 & d 2046 & 808.41 & 907.99 & 698.91 & 772.01 & 892.63 & $80 \%$ & $800 \%$ \\
\hline$[0-132$ & 1000.00 & 2050.00 & $4946.22 \mathrm{a}$ & 957.75 & 957.74 & 957.74 & 957.74 & 957.74 & $-14.80 \%$ & $8.09 \%$ \\
\hline FU-15? & 111200 & ח0 חבכדר & 494623 & 1177.78 & 1206.34 & 1325.15 & 1123.22 & 1067.97 & $-714 \%$ & $682 \%$ \\
\hline$[0-132$ & 1112.00 & $27 \angle 0.00$ & $4940.22 \mathrm{u}$ & 1270.77 & 1270.76 & 1270.76 & 1270.76 & 1270.76 & & $0.02 \%$ \\
\hline FU-15? & 140800 & 420000 & $494622 d$ & 1976.29 & 2032.22 & 2067.48 & 2005.11 & 1839.92 & & \\
\hline & & & $4946.22 \mathrm{a}$ & 1962.21 & 1962.21 & 1962.21 & 1962.20 & 1962.20 & $1.12 \%$ & $3.98 \%$ \\
\hline & & & & & & & & $|x b a r|=$ & $4.23 \%$ & $9.45 \%$ \\
\hline
\end{tabular}

For each count, the number on the top is the observed activity and the number on the bottom is the expected activity.

References:

Draft American National Standard, Performance Criteria for Radiobioassay, ANSI N13.30

Certificate of Calibration, Standard Radionuclide Source, Analytics 
Appendix G

\section{MDA Determination}




\section{Appendix G MDA Determination}

MDA Calculations for High Energy Lung

Arrangement Geometry: High Energy Lung

Analysis Library: INLPLANTLIB.NLB

Energy Calibration Time: 4/5/2011 8:18 AM

Efficiency Calibration Time: 4/5/2011 4:05 PM

Count Live Time: 600 Seconds

Count Start Times:

1. $4 / 6 / 114: 13$ PM
2. $4 / 6 / 114: 25$ PM
3. $4 / 6 / 114: 36$ PM

$\mathrm{MDA}=(4.65 \mathrm{Sb}+3) / \mathrm{KT}$

Where:

$\mathrm{Sb}=$ Standard deviation of the blank phantom counts = SQRT

(gross rate (CPS) xroutine counting time)

$\mathrm{K}=$ Efficiency $X$ Abundance $X 37 \mathrm{~Bq} / \mathrm{nCi}$

$\mathrm{T}=$ Routine counting time in seconds $=600$ seconds

MDA Calculated From Phantom Data

\begin{tabular}{lrrrr}
\multicolumn{1}{c}{ Nuclide } & $\begin{array}{c}\text { Count \#1 } \\
\mathrm{nCi}\end{array}$ & $\begin{array}{c}\text { Count \#2 } \\
\mathrm{nCi}\end{array}$ & $\begin{array}{c}\text { Count \#3 } \\
\mathrm{nCi}\end{array}$ & $\begin{array}{c}\text { Average MDA } \\
\mathrm{nCi}\end{array}$ \\
\hline $\mathrm{K}-40$ & 42.501 & 50.186 & 4.641 & 32.4 \\
$\mathrm{Cr}-51$ & 16.81 & 15.347 & 14.957 & 15.7 \\
$\mathrm{Mn}-54$ & 2.207 & 2.207 & 2.0673 & 2.2 \\
$\mathrm{Co}-57$ & 3.2975 & 3.1373 & 3.0256 & 3.2 \\
$\mathrm{Co}-58$ & 2.6436 & 2.4218 & 2.3016 & 2.5 \\
$\mathrm{Fe}-59$ & 4.4446 & 3.757 & 3.2915 & 3.8 \\
$\mathrm{Co}-60$ & 1.9839 & 1.8735 & 1.6957 & 1.9 \\
$\mathrm{Zn}-65$ & 3.77664 & 6.2281 & 5.9488 & 5.3 \\
$\mathrm{Nb}-95$ & 2.199 & 1.8024 & 2.0767 & 2.0 \\
$\mathrm{Zr}-95$ & 3.9342 & 3.4803 & 3.4803 & 3.6 \\
$\mathrm{Ru}-106$ & 23.623 & 24.415 & 24.415 & 24.2 \\
$\mathrm{Ag}-110 \mathrm{~m}$ & 1.8338 & 2.6492 & 2.5633 & 2.3 \\
$\mathrm{I}-131$ & 2.4636 & 2.4197 & 2.5067 & 2.5 \\
$\mathrm{I}-133$ & 1.9027 & 2.4317 & 2.2709 & 2.2 \\
$\mathrm{Cs}-134$ & 1.8758 & 1.761 & 2.6834 & 2.1 \\
$\mathrm{Cs}-137$ & 2.8555 & 3.304 & 2.6524 & 2.9 \\
$\mathrm{Ba}-140$ & 6.9931 & 9.5074 & 8.2184 & 8.2 \\
La-140 & 1.8059 & 2.3195 & 1.8059 & 2.0 \\
$\mathrm{Ce}-141$ & 5.0959 & 5.4244 & 5.2862 & 5.0 \\
Ce-144 & 23.401 & 25.41 & 25.596 & \\
Eu-152 & 8.01 & 6.6483 & 6.2201 &
\end{tabular}




\section{Analysis Report - Lung Test [999-99-9996]}

\section{SOURCE INFORMATION}

Primary ID

999-99-9996

Last Name

Test

First Name

Lung

Height (in)

Weight (lb)

Chest Wall Thickness (cm)

\section{COUNTER INFORMATION}

Counter Name
Counter Location
Configuration Name

\section{COUNT INFORMATION}

Count Operation

Count Reason

Frequency

Comment

Intake Date

Acquisition Started

Acquisition Time

Operator Name

Count Status

Primary Review

Secondary Review
Accuscan II

Battelle

Lung - High Energy

\section{DETECTOR COUNT RATE REPORT}

\begin{tabular}{|l|c|c|c|c|c|l|}
\hline Detector Name & $\begin{array}{c}\text { Count } \\
\text { Rate }\end{array}$ & $\begin{array}{c}\text { Count } \\
\text { Rate }\end{array}$ & Live Time & $\begin{array}{c}\text { Analyze } \\
\text { d }\end{array}$ & Analyzed Date & File Name \\
\hline Left & 16.12 & $1-4096$ & 600.0 & No & & ef0a51021613.cnf \\
\hline Right & 17.30 & $1-4096$ & 600.0 & No & & aac71c231613.cnf \\
\hline Summed & 33.37 & $1-4096$ & 600.0 & Yes & $4 / 6 / 20114: 13: 01$ PM & 5b8312b31613.cnf \\
\hline
\end{tabular}

Primary Review by:

Date:
Individual 10 minutes

Routine operation

Lung MDA Count \#1

4/6/2011 4:13:10 PM

601.47

David Georgeson

Not Reviewed

No

No 


\section{DETECTOR GROUP INFORMATION}

Detector Group Name
Detector Group Configuration
Analysis Date
Energy Calibration
Efficiency Calibration
Multi Curve
Source Distribution
Analysis Sequence File
Bkg Acq used for area correction
Nuclide Identification
Nuclide Identification Library
Tentative NID Library
Peak Search Library
Analysis Limits (channels)
Energy Tolerance (FWHM)
Comment

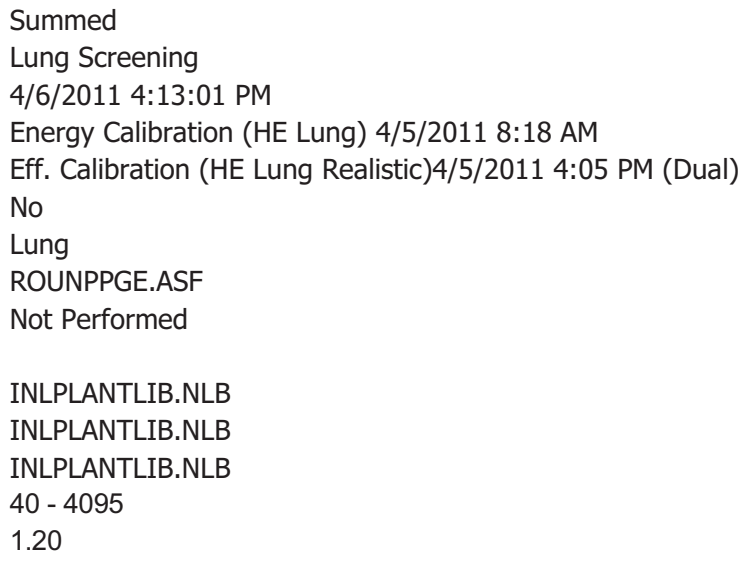

PEAK SEARCH RESULTS FOR Accuscan II <Summed>

\begin{tabular}{|c|c|c|c|c|c||l|l|l|l|}
\hline \multicolumn{9}{|c||}{ Peak Analysis } & \multicolumn{4}{c|}{ Nuclide Information } \\
\hline & No & $\begin{array}{l}\text { Peak } \\
\text { Centroid }\end{array}$ & $\begin{array}{l}\text { Energy } \\
\text { (keV) }\end{array}$ & $\begin{array}{l}\text { Net Peak } \\
\text { Area }\end{array}$ & $\begin{array}{l}\text { Continuum } \\
\text { Counts }\end{array}$ & $\begin{array}{l}\text { Nuclide } \\
\text { Name }\end{array}$ & Activity (nCi) & $\begin{array}{l}\text { Error (1SD) } \\
\text { (15ield } \\
\text { (\%) }\end{array}$ \\
\hline & 1 & 3162.11 & 1460.82 & $5.25 \mathrm{E}+001$ & $6.50 \mathrm{E}+000$ & $\mathrm{~K}-40$ & $8.121071 \mathrm{E}+001$ & $17.19 \%$ & 10.67 \\
\hline
\end{tabular}

For unknown peaks we assume yield to be $100 \%$ and no decay correction is performed.

$M=$ First peak in a multiplet region

$\mathrm{m}=$ Other peak in a multiplet region

$\mathrm{F}=$ Fitted singlet

NUCLIDE RESULTS FOR Accuscan II <Summed>

\begin{tabular}{|l|l|l|l|r|r|l|l|}
\hline & $\begin{array}{l}\text { Nuclide } \\
\text { Name }\end{array}$ & $\begin{array}{l}\text { Id } \\
\text { Confidence }\end{array}$ & $\begin{array}{l}\text { Wt Mean } \\
\text { Activity (nCi) }\end{array}$ & Error (1SD) & Action Level 1 & Action Level 2 & MDA (nCi) \\
\hline K-40 & 1.000 & $8.121071 \mathrm{E}+001$ & $17.19 \%$ & Not Performed & Not Performed & $4.250 \mathrm{E}+001$ \\
\hline
\end{tabular}

$?=$ Nuclide is part of an undetermined solution

$\mathrm{X}=$ Nuclide rejected by the interference analysis

@ = Nuclide contains energy lines not used in Weighted Mean Activity

! = Nuclide was corrected for parent/daughter

\section{NUCLIDE MDA RESULTS FOR Accuscan II <Summed>}

\begin{tabular}{|c|c|c|c|c|c|c|}
\hline & $\begin{array}{l}\text { Nuclide } \\
\text { Name }\end{array}$ & Energy (keV) & Yield (\%) & $\begin{array}{l}\text { Line MDA } \\
\text { (nCi) }\end{array}$ & $\begin{array}{l}\text { Nuclide MDA } \\
\text { (nCi) }\end{array}$ & Activity (nCi) \\
\hline \multirow[t]{2}{*}{+} & $\mathrm{K}-40$ & 1460.81 * & 10.67 & $4.2501 \mathrm{E}+001$ & $4.2501 \mathrm{E}+001$ & $8.1211 \mathrm{E}+001$ \\
\hline & CR-51 & 320.08 & 9.83 & $1.6810 \mathrm{E}+001$ & $1.6810 \mathrm{E}+001$ & $-3.3864 E+000$ \\
\hline & $M N-54$ & 834.83 & 99.97 & $2.2070 \mathrm{E}+000$ & $2.2070 \mathrm{E}+000$ & -8.9644E-001 \\
\hline & \multirow[t]{2}{*}{ CO-57 } & 122.06 & 85.51 & $3.2975 E+000$ & \multirow[t]{2}{*}{$3.2975 E+000$} & $-1.9838 \mathrm{E}-001$ \\
\hline & & 136.48 & 10.60 & $2.3105 E+001$ & & $-1.1639 E+001$ \\
\hline & CO-58 & 810.76 & 99.40 & $2.6436 \mathrm{E}+000$ & $2.6436 \mathrm{E}+000$ & $2.0342 \mathrm{E}-001$ \\
\hline \multirow{3}{*}{\multicolumn{2}{|c|}{ FE-59 }} & 142.65 & 1.03 & $2.3890 \mathrm{E}+002$ & \multirow[t]{3}{*}{$4.4446 \mathrm{E}+000$} & $2.8436 \mathrm{E}+001$ \\
\hline & & 192.34 & 3.11 & $6.8361 \mathrm{E}+001$ & & $-2.1492 \mathrm{E}+001$ \\
\hline & & 1099.22 & 56.50 & $4.4446 \mathrm{E}+000$ & & $-4.6520 \mathrm{E}-001$ \\
\hline
\end{tabular}




\begin{tabular}{|c|c|c|c|c|c|}
\hline & 1291.56 & 43.20 & $6.5678 \mathrm{E}+000$ & & 5.1558E-001 \\
\hline \multirow[t]{2}{*}{$\mathrm{CO}-60$} & 1173.22 & 100.00 & $1.9839 \mathrm{E}+000$ & \multirow[t]{2}{*}{$1.9839 E+000$} & $-6.8933 E-002$ \\
\hline & 1332.49 & 100.00 & $2.4602 \mathrm{E}+000$ & & $-4.1886 E-001$ \\
\hline ZN-65 & 1115.52 & 50.75 & $3.7664 \mathrm{E}+000$ & $3.7664 \mathrm{E}+000$ & $-1.5704 \mathrm{E}+000$ \\
\hline NB-95 & 765.79 & 99.81 & $2.1990 E+000$ & $2.1990 E+000$ & $-6.0447 \mathrm{E}-001$ \\
\hline \multirow[t]{2}{*}{ ZR-95 } & 724.18 & 43.70 & $5.5354 \mathrm{E}+000$ & \multirow[t]{2}{*}{$3.9342 \mathrm{E}+000$} & -3.8537E-001 \\
\hline & 756.72 & 55.30 & $3.9342 \mathrm{E}+000$ & & $-2.7321 \mathrm{E}-001$ \\
\hline
\end{tabular}

User: David Georgeson

Database: InVivo Server: 127.0.0.1 
Analysis Report - Lung Test [999-99-9996]

NUCLIDE MDA RESULTS FOR Accuscan II <Summed>

\begin{tabular}{|c|c|c|c|c|c|}
\hline \begin{tabular}{|l|} 
Nuclide \\
Name
\end{tabular} & Energy (keV) & Yield (\%) & $\begin{array}{l}\text { Line MDA } \\
(\mathrm{nCi})\end{array}$ & $\begin{array}{l}\text { Nuclide MDA } \\
\text { (nCi) }\end{array}$ & Activity (nCi) \\
\hline \multirow[t]{2}{*}{$R U-106$} & 621.84 & 9.80 & $2.3623 \mathrm{E}+001$ & \multirow[t]{2}{*}{$2.3623 \mathrm{E}+001$} & $7.4155 \mathrm{E}+000$ \\
\hline & 1050.47 & 1.73 & $1.1878 \mathrm{E}+002$ & & $2.5737 \mathrm{E}+001$ \\
\hline \multirow[t]{15}{*}{ AG-110M } & 446.80 & 3.64 & $6.2256 \mathrm{E}+001$ & \multirow[t]{15}{*}{$1.8338 \mathrm{E}+000$} & $-1.6419 \mathrm{E}+001$ \\
\hline & 620.35 & 2.77 & $8.3417 \mathrm{E}+001$ & & $7.8075 \mathrm{E}+000$ \\
\hline & 657.75 & 94.40 & $1.8338 \mathrm{E}+000$ & & $-5.0383 E-001$ \\
\hline & 677.61 & 10.68 & $2.5434 \mathrm{E}+001$ & & $2.4953 E+000$ \\
\hline & 686.99 & 6.47 & $2.9537 E+001$ & & $-7.4467 \mathrm{E}+000$ \\
\hline & 706.67 & 16.68 & $1.5326 \mathrm{E}+001$ & & 1.1917E+000 \\
\hline & 744.26 & 4.64 & $3.0857 \mathrm{E}+001$ & & $-6.4329 \mathrm{E}+000$ \\
\hline & 763.93 & 22.28 & $1.0348 \mathrm{E}+001$ & & $9.1058 \mathrm{E}-001$ \\
\hline & 818.02 & 7.30 & $3.1539 E+001$ & & $1.3689 \mathrm{E}+000$ \\
\hline & 884.67 & 72.60 & $3.3538 \mathrm{E}+000$ & & $-2.9113 \mathrm{E}-002$ \\
\hline & 937.48 & 34.20 & $6.5604 \mathrm{E}+000$ & & -5.1500 E-001 \\
\hline & 1384.27 & 24.26 & $9.3312 E+000$ & & $-5.4037 \mathrm{E}-001$ \\
\hline & 1475.76 & 3.97 & $4.0190 \mathrm{E}+001$ & & $-1.4689 \mathrm{E}+001$ \\
\hline & 1505.00 & 13.06 & $1.2444 \mathrm{E}+001$ & & $0.0000 \mathrm{E}+000$ \\
\hline & 1562.27 & 1.18 & $1.4288 \mathrm{E}+002$ & & $-5.2223 \mathrm{E}+001$ \\
\hline \multirow[t]{5}{*}{$\mid-131$} & 80.18 & 2.62 & $1.2173 \mathrm{E}+002$ & \multirow[t]{5}{*}{$2.4636 \mathrm{E}+000$} & $-6.9453 \mathrm{E}+000$ \\
\hline & 284.30 & 6.05 & $2.9737 \mathrm{E}+001$ & & $1.0409 \mathrm{E}+001$ \\
\hline & 364.48 & 81.20 & $2.4636 \mathrm{E}+000$ & & 1.3747E-001 \\
\hline & 636.97 & 7.26 & $2.4824 \mathrm{E}+001$ & & $-3.8246 \mathrm{E}+000$ \\
\hline & 722.89 & 1.80 & $1.3421 \mathrm{E}+002$ & & $1.6965 \mathrm{E}+001$ \\
\hline \multirow[t]{2}{*}{$\mid-133$} & 529.87 & 86.30 & $1.9027 \mathrm{E}+000$ & \multirow[t]{2}{*}{$1.9027 \mathrm{E}+000$} & $-3.0830 \mathrm{E}-001$ \\
\hline & 875.33 & 4.47 & $5.6887 \mathrm{E}+001$ & & $9.8727 \mathrm{E}+000$ \\
\hline \multirow[t]{9}{*}{ CS-134 } & 475.35 & 1.46 & $1.4287 \mathrm{E}+002$ & \multirow[t]{9}{*}{$1.8758 \mathrm{E}+000$} & $-2.2047 \mathrm{E}+001$ \\
\hline & 563.23 & 8.38 & $2.8747 E+001$ & & $4.7450 \mathrm{E}+000$ \\
\hline & 569.32 & 15.43 & $1.5751 \mathrm{E}+001$ & & $4.3228 \mathrm{E}+000$ \\
\hline & 604.70 & 97.60 & $1.8758 \mathrm{E}+000$ & & $-6.5132 \mathrm{E}-002$ \\
\hline & 795.84 & 85.40 & $2.1666 \mathrm{E}+000$ & & 3.6713E-001 \\
\hline & 801.93 & 8.73 & $3.2147 \mathrm{E}+001$ & & $6.9899 \mathrm{E}+000$ \\
\hline & 1038.57 & 1.00 & $2.5744 \mathrm{E}+002$ & & $-1.2620 \mathrm{E}+001$ \\
\hline & 1167.94 & 1.80 & $1.7348 \mathrm{E}+002$ & & $1.7810 \mathrm{E}+001$ \\
\hline & 1365.15 & 3.04 & $6.2894 \mathrm{E}+001$ & & $-5.3690 \mathrm{E}+001$ \\
\hline CS-137 & 661.65 & 85.12 & $2.8555 E+000$ & $2.8555 E+000$ & $2.6726 \mathrm{E}-001$ \\
\hline \multirow[t]{4}{*}{ BA-140 } & 162.64 & 6.70 & $3.7456 \mathrm{E}+001$ & \multirow[t]{4}{*}{$6.9931 E+000$} & $4.7712 \mathrm{E}+000$ \\
\hline & 304.84 & 4.50 & $4.1816 \mathrm{E}+001$ & & $1.3718 \mathrm{E}+001$ \\
\hline & 423.70 & 3.20 & $5.2260 \mathrm{E}+001$ & & $3.3110 \mathrm{E}+000$ \\
\hline & 437.55 & 2.00 & $8.8916 \mathrm{E}+001$ & & $-3.2670 \mathrm{E}+001$ \\
\hline
\end{tabular}




\begin{tabular}{|c|c|c|c|c|c|}
\hline & 537.32 & 25.00 & $6.9931 \mathrm{E}+000$ & & $-1.6923 E+000$ \\
\hline \multirow[t]{7}{*}{ LA-140 } & 328.77 & 20.50 & $8.5857 \mathrm{E}+000$ & \multirow[t]{7}{*}{$1.8059 \mathrm{E}+000$} & $2.7428 \mathrm{E}-001$ \\
\hline & 432.53 & 2.94 & $5.9868 \mathrm{E}+001$ & & $1.0921 \mathrm{E}+001$ \\
\hline & 487.03 & 45.50 & $5.1518 \mathrm{E}+000$ & & $1.3401 \mathrm{E}+000$ \\
\hline & 751.79 & 4.40 & $3.2785 \mathrm{E}+001$ & & $-6.4552 E+000$ \\
\hline & 815.85 & 23.50 & $9.7791 \mathrm{E}+000$ & & -1.9807E-001 \\
\hline & 867.82 & 5.63 & $3.1898 \mathrm{E}+001$ & & $-5.9245 E+000$ \\
\hline & 919.63 & 2.88 & $7.1209 \mathrm{E}+001$ & & $-1.4077 \mathrm{E}+001$ \\
\hline
\end{tabular}

User: David Georgeson

Database: InVivo Server: 127.0.0.1 
NUCLIDE MDA RESULTS FOR Accuscan II <Summed>

\begin{tabular}{|c|c|c|c|c|c|}
\hline \begin{tabular}{|l|} 
Nuclide \\
Name
\end{tabular} & Energy (keV) & Yield (\%) & $\begin{array}{l}\text { Line MDA } \\
\text { (nCi) }\end{array}$ & $\begin{array}{l}\text { Nuclide MDA } \\
\text { (nCi) }\end{array}$ & Activity (nCi) \\
\hline \multirow[t]{2}{*}{ LA-140 } & 925.24 & 7.09 & $2.9048 E+001$ & \multirow[t]{2}{*}{$1.8059 \mathrm{E}+000$} & $-3.2815 E+000$ \\
\hline & 1596.49 & 95.49 & $1.8059 \mathrm{E}+000$ & & $1.8858 \mathrm{E}-001$ \\
\hline CE-141 & 145.44 & 48.40 & $5.0959 \mathrm{E}+000$ & $5.0959 \mathrm{E}+000$ & 7.1776E-002 \\
\hline \multirow[t]{2}{*}{ CE-144 } & 80.11 & 1.60 & $1.9941 \mathrm{E}+002$ & \multirow[t]{2}{*}{$2.3401 \mathrm{E}+001$} & $-1.1377 E+001$ \\
\hline & 133.54 & 10.80 & $2.3401 \mathrm{E}+001$ & & 1.2105E-001 \\
\hline \multirow[t]{11}{*}{ EU-152 } & 121.78 & 28.40 & $1.0016 \mathrm{E}+001$ & \multirow[t]{11}{*}{$8.0100 \mathrm{E}+000$} & $4.8692 \mathrm{E}+000$ \\
\hline & 244.69 & 7.49 & $2.5366 \mathrm{E}+001$ & & $-1.5236 \mathrm{E}+000$ \\
\hline & 344.27 & 26.50 & $8.0100 \mathrm{E}+000$ & & $-3.5716 E-001$ \\
\hline & 411.11 & 2.21 & $9.5256 \mathrm{E}+001$ & & $1.7387 \mathrm{E}+001$ \\
\hline & 443.98 & 3.11 & $6.6431 \mathrm{E}+001$ & & $-1.0159 E+001$ \\
\hline & 778.89 & 12.74 & $1.6473 \mathrm{E}+001$ & & $1.6151 \mathrm{E}+000$ \\
\hline & 867.32 & 4.16 & $4.3153 \mathrm{E}+001$ & & $-1.0019 E+001$ \\
\hline & 964.01 & 14.40 & $1.5889 \mathrm{E}+001$ & & $3.2568 \mathrm{E}+000$ \\
\hline & 1085.78 & 10.00 & $2.4894 \mathrm{E}+001$ & & $3.2569 \mathrm{E}+000$ \\
\hline & 1112.02 & 13.30 & $2.1522 \mathrm{E}+001$ & & $4.3592 \mathrm{E}+000$ \\
\hline & 1407.95 & 20.70 & $9.4862 \mathrm{E}+000$ & & $-1.9281 E+000$ \\
\hline
\end{tabular}

$+=$ Nuclide Identified during the nuclide identification

$*$ = Energy Line found in the spectrum

\section{Summed}

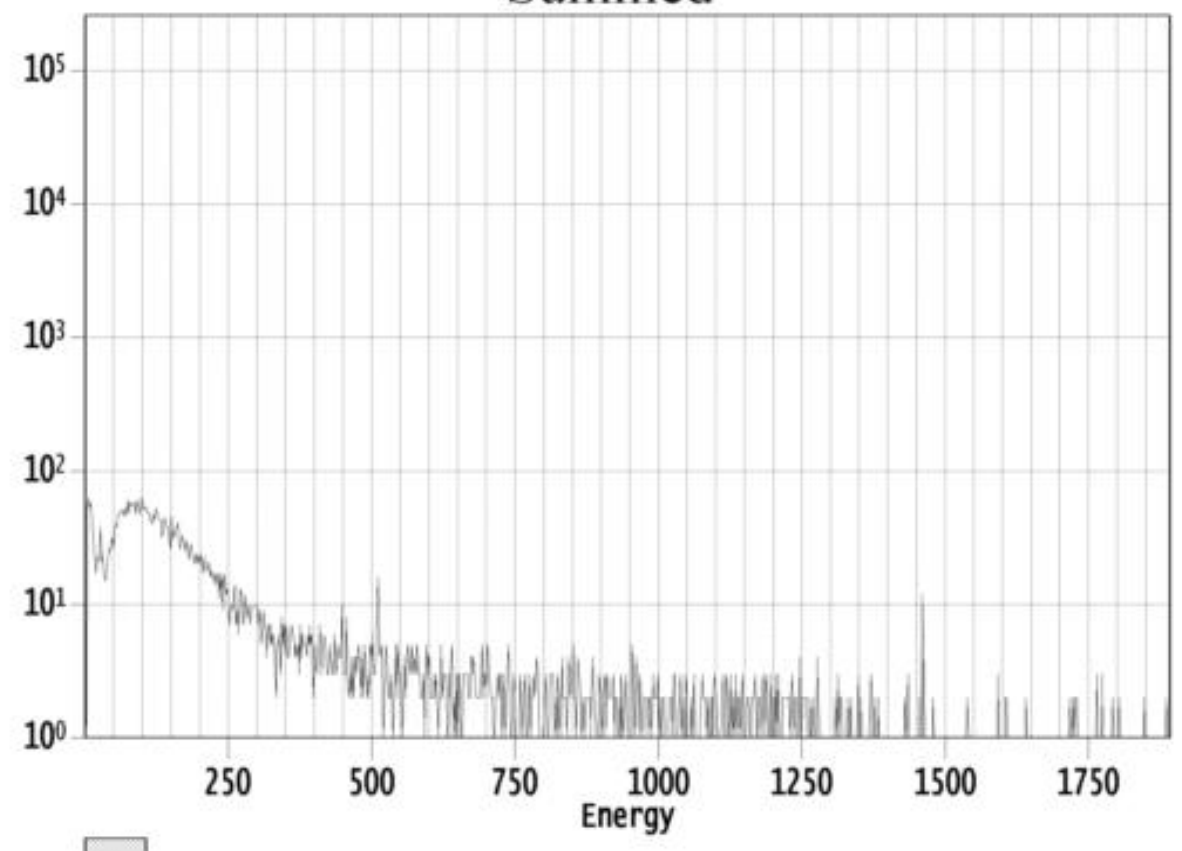

ROI Type: 1 


\section{Analysis Report - Lung Test [999-99-9996]}

\section{SOURCE INFORMATION}

Primary ID

999-99-9996

Last Name

Test

First Name

Lung

Height (in)

Weight (lb)

Chest Wall Thickness (cm)

\section{COUNTER INFORMATION}

Counter Name
Counter Location
Configuration Name

\section{COUNT INFORMATION}

Count Operation

Count Reason

Frequency

Comment

Intake Date

Acquisition Started

Acquisition Time

Operator Name

Count Status

Primary Review

Secondary Review
Accuscan II

Battelle

Lung - High Energy

\section{DETECTOR COUNT RATE REPORT}

\begin{tabular}{|l|r|r|r|c|c|l|}
\hline \multicolumn{1}{|c|}{ Detector Name } & $\begin{array}{c}\text { Count } \\
\text { Rate }\end{array}$ & $\begin{array}{c}\text { Count Rate } \\
\text { Range }\end{array}$ & Live Time & Analyzed & Analyzed Date & File Name \\
\hline Right & 17.65 & $1-4096$ & 600.0 & No & & 7bf99bdf1625.cnf \\
\hline Left & 15.93 & $1-4096$ & 600.0 & No & & d129dcf51625.cnf \\
\hline Summed & 33.57 & $1-4096$ & 600.0 & Yes & $4 / 6 / 20114: 25: 01$ & 7ba6552a1625.cnf \\
\hline
\end{tabular}

Primary Review by:

Date:

User: David Georgeson
Individual 10 minutes

Routine operation

Lung MDA Count \#2

4/6/2011 4:25:09 PM

601.35

David Georgeson

Not Reviewed

No

No 


\section{DETECTOR GROUP INFORMATION}

Detector Group Name
Detector Group Configuration
Analysis Date
Energy Calibration
Efficiency Calibration
Multi Curve
Source Distribution
Analysis Sequence File
Bkg Acq used for area correction
Nuclide Identification
Nuclide Identification Library
Tentative NID Library
Peak Search Library
Analysis Limits (channels)
Energy Tolerance (FWHM)
Comment

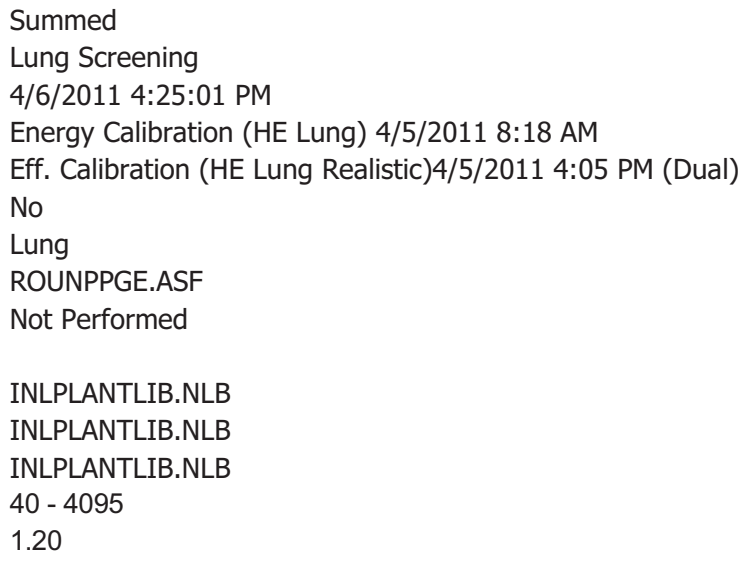

PEAK SEARCH RESULTS FOR Accuscan II <Summed>

\begin{tabular}{|c|c|c|c|c|c|c|c|c|}
\hline \multicolumn{5}{|c|}{ Peak Analysis } & \multicolumn{4}{|c|}{ Nuclide Information } \\
\hline No & \begin{tabular}{|l|} 
Peak \\
Centroid
\end{tabular} & $\begin{array}{l}\text { Energy } \\
(\mathrm{keV})\end{array}$ & $\begin{array}{l}\text { Net Peak } \\
\text { Area }\end{array}$ & $\begin{array}{l}\text { Continuum } \\
\text { Counts }\end{array}$ & \begin{tabular}{|l}
$\begin{array}{l}\text { Nuclide } \\
\text { Name }\end{array}$ \\
\end{tabular} & Activity (nCi) & Error (1SD) & $\begin{array}{l}\text { Yield } \\
(\%)\end{array}$ \\
\hline 1 & 3161.97 & 1460.75 & $3.85 \mathrm{E}+001$ & $9.51 \mathrm{E}+000$ & $K-40$ & $5.953245 \mathrm{E}+0$ & $23.13 \%$ & 10.67 \\
\hline
\end{tabular}

For unknown peaks we assume yield to be $100 \%$ and no decay correction is performed.

$M=$ First peak in a multiplet region

$\mathrm{m}=$ Other peak in a multiplet region

$\mathrm{F}=$ Fitted singlet

NUCLIDE RESULTS FOR Accuscan II <Summed>

\begin{tabular}{|l|l|l|l|r|r|r|r|}
\hline & $\begin{array}{l}\text { Nuclide } \\
\text { Name }\end{array}$ & $\begin{array}{l}\text { Id } \\
\text { Confidence }\end{array}$ & $\begin{array}{l}\text { Wt Mean } \\
\text { Activity (nCi) }\end{array}$ & Error (1SD) & Action Level 1 & Action Level 2 & MDA (nCi) \\
\hline K-40 & 1.000 & $5.953245 \mathrm{E}+001$ & $23.13 \%$ & Not Performed & Not Performed & $5.019 \mathrm{E}+001$ \\
\hline
\end{tabular}

$?=$ Nuclide is part of an undetermined solution

$\mathrm{X}=$ Nuclide rejected by the interference analysis

@ = Nuclide contains energy lines not used in Weighted Mean Activity

! = Nuclide was corrected for parent/daughter

\section{NUCLIDE MDA RESULTS FOR Accuscan II <Summed>}

\begin{tabular}{|c|c|c|c|c|c|c|}
\hline & $\begin{array}{l}\text { Nuclide } \\
\text { Name }\end{array}$ & Energy (keV) & Yield (\%) & $\begin{array}{l}\text { Line MDA } \\
(\mathrm{nCi})\end{array}$ & $\begin{array}{l}\text { Nuclide MDA } \\
\text { (nCi) }\end{array}$ & Activity (nCi) \\
\hline \multirow[t]{2}{*}{+} & $\mathrm{K}-40$ & $1460.81 *$ & 10.67 & $5.0186 \mathrm{E}+001$ & $5.0186 \mathrm{E}+001$ & $5.9532 E+001$ \\
\hline & CR-51 & 320.08 & 9.83 & $1.5347 \mathrm{E}+001$ & $1.5347 E+001$ & $-1.9591 E+000$ \\
\hline & MN-54 & 834.83 & 99.97 & $2.2070 \mathrm{E}+000$ & $2.2070 E+000$ & $-3.7867 \mathrm{E}-001$ \\
\hline & \multirow[t]{2}{*}{ CO-57 } & 122.06 & 85.51 & $3.1373 \mathrm{E}+000$ & \multirow[t]{2}{*}{$3.1373 \mathrm{E}+000$} & 5.0330E-001 \\
\hline & & 136.48 & 10.60 & $2.4432 \mathrm{E}+001$ & & $-2.2843 E+000$ \\
\hline & CO-58 & 810.76 & 99.40 & $2.4218 \mathrm{E}+000$ & $2.4218 \mathrm{E}+000$ & $2.1311 \mathrm{E}-001$ \\
\hline & \multirow[t]{3}{*}{ FE-59 } & 142.65 & 1.03 & $2.4776 \mathrm{E}+002$ & \multirow[t]{3}{*}{$3.7570 \mathrm{E}+000$} & $2.4033 E+001$ \\
\hline & & 192.34 & 3.11 & $6.7599 E+001$ & & $2.1597 E+001$ \\
\hline & & 1099.22 & 56.50 & $3.7570 \mathrm{E}+000$ & & 1.1630E-001 \\
\hline
\end{tabular}




\begin{tabular}{|r|r|r|r|r|r|r|}
\hline & & 1291.56 & 43.20 & $4.9461 \mathrm{E}+000$ & & $-1.1457 \mathrm{E}+000$ \\
\hline \multirow{2}{*}{ CO-60 } & 1173.22 & 100.00 & $2.8123 \mathrm{E}+000$ & $1.8735 \mathrm{E}+000$ & $7.3857 \mathrm{E}-001$ \\
\cline { 3 - 5 } & 1332.49 & 100.00 & $1.8735 \mathrm{E}+000$ & & $-3.8079 \mathrm{E}-001$ \\
\hline & ZN-65 & 1115.52 & 50.75 & $6.2281 \mathrm{E}+000$ & $6.2281 \mathrm{E}+000$ & $2.8791 \mathrm{E}-001$ \\
\hline \multirow{2}{*}{ NB-95 } & 765.79 & 99.81 & $1.8024 \mathrm{E}+000$ & $1.8024 \mathrm{E}+000$ & $-1.2217 \mathrm{E}+000$ \\
\hline \multirow{2}{*}{ ZR-95 } & 724.18 & 43.70 & $5.7518 \mathrm{E}+000$ & $3.4803 \mathrm{E}+000$ & $2.2311 \mathrm{E}+000$ \\
\cline { 3 - 5 } & & 756.72 & 55.30 & $3.4803 \mathrm{E}+000$ & & $-1.8214 \mathrm{E}-001$ \\
\hline
\end{tabular}

User: David Georgeson

Database: InVivo Server: 127.0.0.1 
Analysis Report - Lung Test [999-99-9996]

NUCLIDE MDA RESULTS FOR Accuscan II <Summed>

\begin{tabular}{|c|c|c|c|c|c|}
\hline \begin{tabular}{|l|} 
Nuclide \\
Name
\end{tabular} & Energy (keV) & Yield (\%) & $\begin{array}{l}\text { Line MDA } \\
\text { (nCi) }\end{array}$ & $\begin{array}{l}\text { Nuclide MDA } \\
\text { (nCi) }\end{array}$ & Activity (nCi) \\
\hline \multirow[t]{2}{*}{ RU-106 } & 621.84 & 9.80 & $2.4415 \mathrm{E}+001$ & \multirow[t]{2}{*}{$2.4415 E+001$} & 4.4221E-001 \\
\hline & 1050.47 & 1.73 & $1.5000 \mathrm{E}+002$ & & $3.3091 \mathrm{E}+001$ \\
\hline \multirow[t]{15}{*}{ AG-110M } & 446.80 & 3.64 & $5.1317 \mathrm{E}+001$ & \multirow[t]{15}{*}{$2.6492 \mathrm{E}+000$} & $1.3159 \mathrm{E}+001$ \\
\hline & 620.35 & 2.77 & $8.3417 \mathrm{E}+001$ & & $-1.4414 \mathrm{E}+000$ \\
\hline & 657.75 & 94.40 & $2.6492 E+000$ & & 5.0383E-001 \\
\hline & 677.61 & 10.68 & $1.7705 \mathrm{E}+001$ & & $-2.1079 E+000$ \\
\hline & 686.99 & 6.47 & $3.2910 \mathrm{E}+001$ & & $-4.7462 \mathrm{E}+000$ \\
\hline & 706.67 & 16.68 & $1.4236 \mathrm{E}+001$ & & $1.7475 \mathrm{E}+000$ \\
\hline & 744.26 & 4.64 & $4.6317 \mathrm{E}+001$ & & $-1.0185 E+001$ \\
\hline & 763.93 & 22.28 & $9.8342 E+000$ & & 9.1058E-001 \\
\hline & 818.02 & 7.30 & $2.5852 E+001$ & & $-1.4164 \mathrm{E}+001$ \\
\hline & 884.67 & 72.60 & $2.9669 \mathrm{E}+000$ & & 8.1516E-001 \\
\hline & 937.48 & 34.20 & $7.0036 \mathrm{E}+000$ & & $1.1771 \mathrm{E}+000$ \\
\hline & 1384.27 & 24.26 & $1.0474 \mathrm{E}+001$ & & $-8.1056 \mathrm{E}-002$ \\
\hline & 1475.76 & 3.97 & $5.1622 E+001$ & & $5.2461 \mathrm{E}+000$ \\
\hline & 1505.00 & 13.06 & $1.8700 \mathrm{E}+001$ & & $-7.7972 \mathrm{E}+000$ \\
\hline & 1562.27 & 1.18 & $2.1471 \mathrm{E}+002$ & & $1.4921 \mathrm{E}+001$ \\
\hline \multirow[t]{5}{*}{$\mid-131$} & 80.18 & 2.62 & $1.2795 \mathrm{E}+002$ & \multirow[t]{5}{*}{$2.4197 \mathrm{E}+000$} & $9.8526 \mathrm{E}+000$ \\
\hline & 284.30 & 6.05 & $2.9317 \mathrm{E}+001$ & & $9.1842 \mathrm{E}+000$ \\
\hline & 364.48 & 81.20 & $2.4197 \mathrm{E}+000$ & & 3.0751E-001 \\
\hline & 636.97 & 7.26 & $3.5661 \mathrm{E}+001$ & & $-3.7268 \mathrm{E}+000$ \\
\hline & 722.89 & 1.80 & $1.1684 \mathrm{E}+002$ & & $-2.0285 \mathrm{E}+000$ \\
\hline \multirow[t]{2}{*}{$\mid-133$} & 529.87 & 86.30 & $2.4317 \mathrm{E}+000$ & \multirow[t]{2}{*}{$2.4317 \mathrm{E}+000$} & $-1.2521 E+000$ \\
\hline & 875.33 & 4.47 & $5.6887 E+001$ & & $1.4739 \mathrm{E}+001$ \\
\hline \multirow[t]{9}{*}{ CS-134 } & 475.35 & 1.46 & $1.2231 \mathrm{E}+002$ & \multirow[t]{9}{*}{$1.7610 \mathrm{E}+000$} & $7.1166 \mathrm{E}+000$ \\
\hline & 563.23 & 8.38 & $2.7177 \mathrm{E}+001$ & & $-5.1231 E+000$ \\
\hline & 569.32 & 15.43 & $1.4890 \mathrm{E}+001$ & & 1.1507E+000 \\
\hline & 604.70 & 97.60 & $2.1546 \mathrm{E}+000$ & & 3.4737E-001 \\
\hline & 795.84 & 85.40 & $1.7610 \mathrm{E}+000$ & & $-1.2034 \mathrm{E}+000$ \\
\hline & 801.93 & 8.73 & $3.1028 \mathrm{E}+001$ & & $4.8142 \mathrm{E}+000$ \\
\hline & 1038.57 & 1.00 & $1.5523 E+002$ & & $-4.1016 E+001$ \\
\hline & 1167.94 & 1.80 & $1.2329 \mathrm{E}+002$ & & $1.3358 \mathrm{E}+001$ \\
\hline & 1365.15 & 3.04 & $9.7705 E+001$ & & $3.0680 \mathrm{E}+001$ \\
\hline CS-137 & 661.65 & 85.12 & $3.3034 \mathrm{E}+000$ & $3.3034 \mathrm{E}+000$ & 4.2762E-001 \\
\hline \multirow[t]{4}{*}{ BA-140 } & 162.64 & 6.70 & $3.5275 E+001$ & \multirow[t]{4}{*}{$9.5074 \mathrm{E}+000$} & $3.1119 \mathrm{E}+000$ \\
\hline & 304.84 & 4.50 & $3.6632 E+001$ & & $7.3680 \mathrm{E}+000$ \\
\hline & 423.70 & 3.20 & $5.7335 \mathrm{E}+001$ & & $3.9131 \mathrm{E}+000$ \\
\hline & 437.55 & 2.00 & $8.6033 \mathrm{E}+001$ & & $9.6628 \mathrm{E}+000$ \\
\hline
\end{tabular}




\begin{tabular}{|c|c|c|c|c|c|}
\hline & 537.32 & 25.00 & $9.5074 \mathrm{E}+000$ & & 7.9485E-001 \\
\hline \multirow[t]{7}{*}{ LA-140 } & 328.77 & 20.50 & $8.9064 \mathrm{E}+000$ & \multirow[t]{7}{*}{$2.3195 \mathrm{E}+000$} & 8.0642E-001 \\
\hline & 432.53 & 2.94 & $7.3399 \mathrm{E}+001$ & & $-2.2673 E+000$ \\
\hline & 487.03 & 45.50 & $4.5561 \mathrm{E}+000$ & & -8.5998E-001 \\
\hline & 751.79 & 4.40 & $5.4213 \mathrm{E}+001$ & & $1.1392 \mathrm{E}+000$ \\
\hline & 815.85 & 23.50 & $1.0773 \mathrm{E}+001$ & & 5.8856E-001 \\
\hline & 867.82 & 5.63 & $3.7735 \mathrm{E}+001$ & & $6.5828 \mathrm{E}+000$ \\
\hline & 919.63 & 2.88 & $7.1209 \mathrm{E}+001$ & & $-5.6309 E+000$ \\
\hline
\end{tabular}

User: David Georgeson

Database: InVivo Server: 127.0.0.1 
NUCLIDE MDA RESULTS FOR Accuscan II <Summed>

\begin{tabular}{|c|c|c|c|c|c|}
\hline \begin{tabular}{|l} 
Nuclide \\
Name
\end{tabular} & Energy (keV) & Yield (\%) & $\begin{array}{l}\text { Line MDA } \\
\text { (nCi) }\end{array}$ & $\begin{array}{l}\text { Nuclide MDA } \\
\text { (nCi) }\end{array}$ & Activity (nCi) \\
\hline \multirow[t]{2}{*}{ LA-140 } & 925.24 & 7.09 & $3.7291 \mathrm{E}+001$ & \multirow[t]{2}{*}{$2.3195 E+000$} & $1.2944 \mathrm{E}+001$ \\
\hline & 1596.49 & 95.49 & $2.3195 E+000$ & & $-4.7145 \mathrm{E}-001$ \\
\hline CE-141 & 145.44 & 48.40 & $5.4244 \mathrm{E}+000$ & $5.4244 \mathrm{E}+000$ & 1.3594E-001 \\
\hline \multirow[t]{2}{*}{ CE-144 } & 80.11 & 1.60 & $2.0959 \mathrm{E}+002$ & \multirow[t]{2}{*}{$2.5410 \mathrm{E}+001$} & $1.6140 \mathrm{E}+001$ \\
\hline & 133.54 & 10.80 & $2.5410 \mathrm{E}+001$ & & $7.4528 \mathrm{E}+000$ \\
\hline \multirow[t]{11}{*}{ EU-152 } & 121.78 & 28.40 & $9.3976 \mathrm{E}+000$ & \multirow[t]{11}{*}{$6.6483 \mathrm{E}+000$} & $-2.9954 \mathrm{E}-001$ \\
\hline & 244.69 & 7.49 & $2.4353 E+001$ & & $-1.0519 E+001$ \\
\hline & 344.27 & 26.50 & $6.6483 E+000$ & & 6.4288E-001 \\
\hline & 411.11 & 2.21 & $9.1388 \mathrm{E}+001$ & & $-1.3790 E+001$ \\
\hline & 443.98 & 3.11 & $6.3178 \mathrm{E}+001$ & & $9.3174 \mathrm{E}+000$ \\
\hline & 778.89 & 12.74 & $1.5431 \mathrm{E}+001$ & & $1.0767 \mathrm{E}+000$ \\
\hline & 867.32 & 4.16 & $5.7708 \mathrm{E}+001$ & & $2.1373 \mathrm{E}+001$ \\
\hline & 964.01 & 14.40 & $2.2955 E+001$ & & $8.1964 \mathrm{E}+000$ \\
\hline & 1085.78 & 10.00 & $2.6575 \mathrm{E}+001$ & & $-8.8403 E+000$ \\
\hline & 1112.02 & 13.30 & $1.6094 \mathrm{E}+001$ & & $-1.6191 \mathrm{E}+000$ \\
\hline & 1407.95 & 20.70 & $1.3654 \mathrm{E}+001$ & & $2.0052 \mathrm{E}+000$ \\
\hline
\end{tabular}

$+=$ Nuclide Identified during the nuclide identification

$*=$ Energy Line found in the spectrum

\section{Summed}

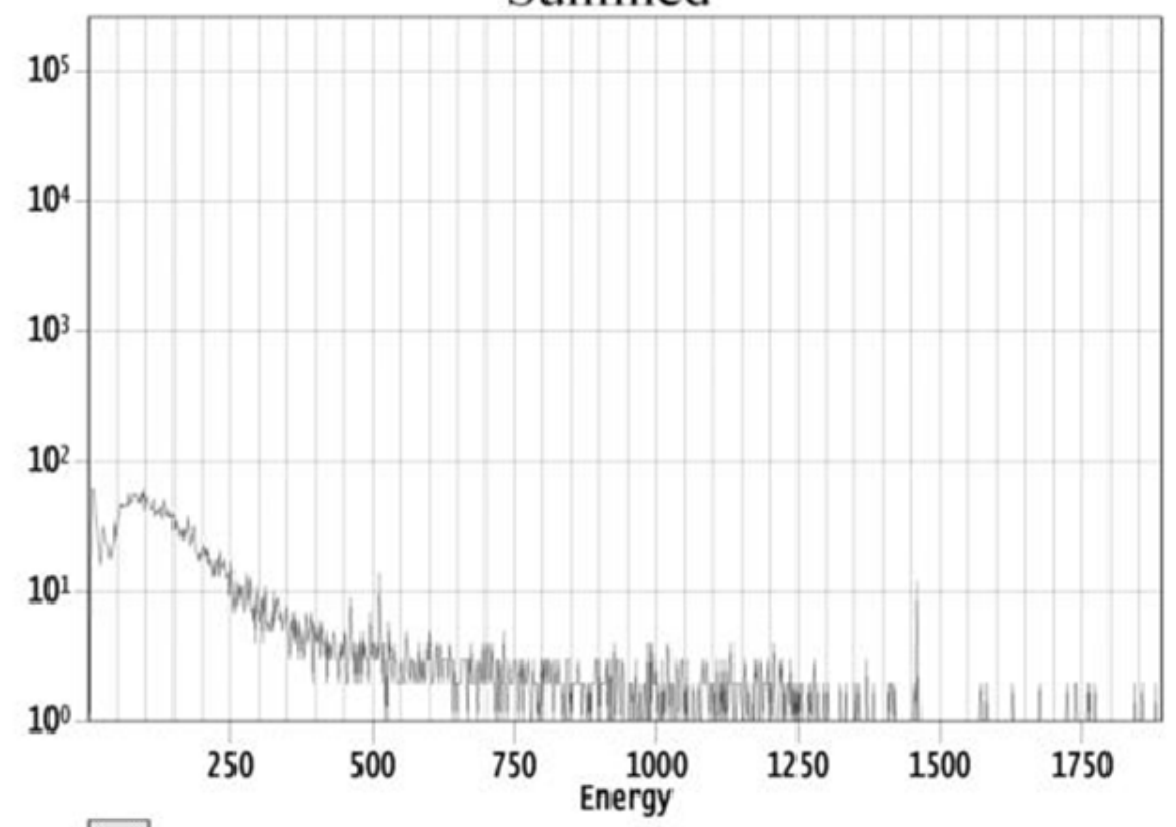

ROI Type: 1 


\section{Analysis Report - Lung Test [999-99-9996]}

\section{SOURCE INFORMATION}

Primary ID

Last Name

First Name

Height (in)

Weight (lb)

Chest Wall Thickness (cm)
999-99-9996

Test

Lung

\section{COUNTER INFORMATION}

Counter Name

Counter Location

Configuration Name
Accuscan II

Battelle

Lung - High Energy

\section{COUNT INFORMATION}

\begin{tabular}{ll}
$\begin{array}{l}\text { Count Operation } \\
\text { Count Reason } \\
\text { Frequency }\end{array}$ & $\begin{array}{l}\text { Individual } 10 \text { minutes } \\
\text { Routine operation }\end{array}$ \\
$\begin{array}{l}\text { Intake Date } \\
\text { Acquisition Started }\end{array}$ & Lung MDA Count \#3 \\
Acquisition Time & $4 / 6 / 20114: 36: 01$ PM \\
Operator Name & 601.49 \\
Count Status & David Georgeson \\
Primary Review & Not Reviewed \\
Secondary Review & No \\
\hline
\end{tabular}

\section{DETECTOR COUNT RATE REPORT}

\begin{tabular}{|l|r|r|r|r|r|l|}
\hline \multicolumn{1}{|c|}{ Detector Name } & $\begin{array}{c}\text { Count } \\
\text { Rate }\end{array}$ & $\begin{array}{c}\text { Count Rate } \\
\text { Range }\end{array}$ & Live Time & Analyzed & Analyzed Date & File Name \\
\hline Left & 16.01 & $1-4096$ & 600.0 & No & & 7bb7cef01635.cnf \\
\hline Right & 17.54 & $1-4096$ & 600.0 & No & & cbaa1b691635.cnf \\
\hline Summed & 33.52 & $1-4096$ & 600.0 & Yes & $4 / 6 / 20114: 35: 53$ PM & $6 c 28 b b d d 1635 . c n f$ \\
\hline
\end{tabular}

Primary Review by:

Date:
Secondary Review by:

Date: 


\title{
DETECTOR GROUP INFORMATION
}

Detector Group Name
Detector Group Configuration
Analysis Date
Energy Calibration
Efficiency Calibration
Multi Curve
Source Distribution
Analysis Sequence File
Bkg Acq used for area correction
Nuclide Identification
Nuclide Identification Library
Tentative NID Library
Peak Search Library
Analysis Limits (channels)
Energy Tolerance (FWHM)
Comment

\author{
Summed \\ Lung Screening \\ 4/6/2011 4:35:53 PM \\ No \\ Lung \\ ROUNPPGE.ASF \\ Not Performed \\ INLPLANTLIB.NLB \\ INLPLANTLIB.NLB \\ INLPLANTLIB.NLB \\ $40-4095$ \\ 1.20
}

Energy Calibration (HE Lung) 4/5/2011 8:18 AM

Eff. Calibration (HE Lung Realistic)4/5/2011 4:05 PM (Dual)

PEAK SEARCH RESULTS FOR Accuscan II <Summed>

\begin{tabular}{|c|c|c|c|c|c||l|l|l|l|}
\hline \multicolumn{9}{|c||}{ Peak Analysis } & \multicolumn{4}{c|}{ Nuclide Information } \\
\hline & No & $\begin{array}{l}\text { Peak } \\
\text { Centroid }\end{array}$ & $\begin{array}{l}\text { Energy } \\
\text { (keV) }\end{array}$ & $\begin{array}{l}\text { Net Peak } \\
\text { Area }\end{array}$ & $\begin{array}{l}\text { Continuum } \\
\text { Counts }\end{array}$ & $\begin{array}{l}\text { Nuclide } \\
\text { Name }\end{array}$ & Activity (nCi) & $\begin{array}{l}\text { Error } \\
\text { (1SD) }\end{array}$ & $\begin{array}{l}\text { Yield } \\
\text { (\%) }\end{array}$ \\
\hline & 1 & 3162.20 & 1460.85 & $6.10 \mathrm{E}+001$ & $0.00 \mathrm{E}+000$ & K-40 & $9.436748 \mathrm{E}+001$ & $12.98 \%$ & 10.67 \\
\hline
\end{tabular}

For unknown peaks we assume yield to be $100 \%$ and no decay correction is performed.

$\mathrm{M}=$ First peak in a multiplet region

$\mathrm{m}=$ Other peak in a multiplet region

$\mathrm{F}=$ Fitted singlet

NUCLIDE RESULTS FOR Accuscan II <Summed>

\begin{tabular}{|l|l|l|l|l|l|l|l|}
\hline & $\begin{array}{l}\text { Nuclide } \\
\text { Name }\end{array}$ & $\begin{array}{l}\text { Id } \\
\text { Confidence }\end{array}$ & $\begin{array}{l}\text { Wt Mean } \\
\text { Activity (nCi) }\end{array}$ & Error (1SD) & Action Level 1 & Action Level 2 & MDA (nCi) \\
\hline K-40 & 1.000 & $9.436748 \mathrm{E}+00$ & $12.98 \%$ & Not Performed & Not Performed & $4.641 \mathrm{E}+000$ \\
\hline
\end{tabular}

$?=$ Nuclide is part of an undetermined solution

$\mathrm{X}=$ Nuclide rejected by the interference analysis

@ = Nuclide contains energy lines not used in Weighted Mean Activity

! = Nuclide was corrected for parent/daughter

\section{NUCLIDE MDA RESULTS FOR Accuscan II <Summed>}

\begin{tabular}{|c|c|c|c|c|c|c|}
\hline & \begin{tabular}{|l} 
Nuclide \\
Name
\end{tabular} & Energy (keV) & Yield (\%) & $\begin{array}{l}\text { Line MDA } \\
(\mathrm{nCi})\end{array}$ & $\begin{array}{l}\text { Nuclide MDA } \\
\text { (nCi) }\end{array}$ & Activity (nCi) \\
\hline \multirow[t]{3}{*}{+} & $\mathrm{K}-40$ & 1460.81 * & 10.67 & $4.6410 \mathrm{E}+000$ & $4.6410 \mathrm{E}+000$ & $9.4367 \mathrm{E}+001$ \\
\hline & CR-51 & 320.08 & 9.83 & $1.4957 E+001$ & $1.4957 \mathrm{E}+001$ & $-4.3824 \mathrm{E}-001$ \\
\hline & MN-54 & 834.83 & 99.97 & $2.0673 E+000$ & $2.0673 \mathrm{E}+000$ & $-4.8686 \mathrm{E}-001$ \\
\hline & \multirow[t]{2}{*}{$\mathrm{CO}-57$} & 122.06 & 85.51 & $3.0256 \mathrm{E}+000$ & \multirow[t]{2}{*}{$3.0256 \mathrm{E}+000$} & $-1.2972 E+000$ \\
\hline & & 136.48 & 10.60 & $2.5101 \mathrm{E}+001$ & & $-2.8910 \mathrm{E}+000$ \\
\hline & CO-58 & 810.76 & 99.40 & $2.3016 \mathrm{E}+000$ & $2.3016 \mathrm{E}+000$ & $-7.3255 E-001$ \\
\hline & \multirow[t]{3}{*}{ FE-59 } & 142.65 & 1.03 & $2.7126 \mathrm{E}+002$ & \multirow[t]{3}{*}{$3.2915 E+000$} & 1.1047E+002 \\
\hline & & 192.34 & 3.11 & $7.6221 \mathrm{E}+001$ & & $2.4573 E+001$ \\
\hline & & 1099.22 & 56.50 & $4.7448 E+000$ & & 4.8182E-001 \\
\hline
\end{tabular}




\begin{tabular}{|c|c|c|c|c|c|}
\hline & 1291.56 & 43.20 & $3.2915 \mathrm{E}+000$ & & -8.5929E-001 \\
\hline \multirow[t]{2}{*}{ CO-60 } & 1173.22 & 100.00 & 1.6957E+000 & \multirow[t]{2}{*}{ 1.6957E+000 } & $-8.9613 \mathrm{E}-001$ \\
\hline & 1332.49 & 100.00 & $2.9104 \mathrm{E}+000$ & & 9.1389E-001 \\
\hline ZN-65 & 1115.52 & 50.75 & $5.9488 \mathrm{E}+000$ & $5.9488 \mathrm{E}+000$ & $4.2168 \mathrm{E}-001$ \\
\hline NB-95 & 765.79 & 99.81 & $2.0767 \mathrm{E}+000$ & $2.0767 E+000$ & $-4.7267 \mathrm{E}-001$ \\
\hline \multirow[t]{2}{*}{ ZR-95 } & 724.18 & 43.70 & $5.0708 \mathrm{E}+000$ & \multirow[t]{2}{*}{$3.4803 E+000$} & 8.1806E-001 \\
\hline & 756.72 & 55.30 & $3.4803 E+000$ & & $-8.1962 \mathrm{E}-001$ \\
\hline
\end{tabular}

User: David Georgeson

Database: InVivo Server: 127.0.0.1 
Analysis Report - Lung Test [999-99-9996]

NUCLIDE MDA RESULTS FOR Accuscan II <Summed>

\begin{tabular}{|c|c|c|c|c|c|}
\hline \begin{tabular}{|l|} 
Nuclide \\
Name
\end{tabular} & Energy (keV) & Yield (\%) & $\begin{array}{l}\text { Line MDA } \\
\text { (nCi) }\end{array}$ & $\begin{array}{l}\text { Nuclide MDA } \\
\text { (nCi) }\end{array}$ & Activity (nCi) \\
\hline \multirow[t]{2}{*}{ RU-106 } & 621.84 & 9.80 & $2.4415 \mathrm{E}+001$ & \multirow[t]{2}{*}{$2.4415 E+001$} & $7.7386 \mathrm{E}+000$ \\
\hline & 1050.47 & 1.73 & $1.0582 \mathrm{E}+002$ & & $-1.2256 \mathrm{E}+001$ \\
\hline \multirow[t]{15}{*}{ AG-110M } & 446.80 & 3.64 & $5.8419 \mathrm{E}+001$ & \multirow[t]{15}{*}{$2.5633 \mathrm{E}+000$} & 1.1717E+001 \\
\hline & 620.35 & 2.77 & 7.7483E+001 & & $-3.1230 \mathrm{E}+001$ \\
\hline & 657.75 & 94.40 & $2.5633 E+000$ & & $1.3288 \mathrm{E}-001$ \\
\hline & 677.61 & 10.68 & $1.9727 \mathrm{E}+001$ & & $-3.6164 \mathrm{E}+000$ \\
\hline & 686.99 & 6.47 & $3.1276 \mathrm{E}+001$ & & $-2.8960 \mathrm{E}+000$ \\
\hline & 706.67 & 16.68 & $1.0964 \mathrm{E}+001$ & & $-2.1039 E+000$ \\
\hline & 744.26 & 4.64 & $5.3201 \mathrm{E}+001$ & & $2.3392 \mathrm{E}+000$ \\
\hline & 763.93 & 22.28 & $1.0348 \mathrm{E}+001$ & & $2.5041 \mathrm{E}+000$ \\
\hline & 818.02 & 7.30 & $2.5852 E+001$ & & $-1.5770 \mathrm{E}+001$ \\
\hline & 884.67 & 72.60 & $2.7490 \mathrm{E}+000$ & & $-3.5712 E-001$ \\
\hline & 937.48 & 34.20 & $7.0036 \mathrm{E}+000$ & & $0.0000 \mathrm{E}+000$ \\
\hline & 1384.27 & 24.26 & $1.2391 \mathrm{E}+001$ & & 9.7267E-001 \\
\hline & 1475.76 & 3.97 & $8.5611 \mathrm{E}+001$ & & $1.9486 \mathrm{E}+001$ \\
\hline & 1505.00 & 13.06 & $3.8986 \mathrm{E}+000$ & & $0.0000 \mathrm{E}+000$ \\
\hline & 1562.27 & 1.18 & $1.8353 \mathrm{E}+002$ & & 1.1191E+001 \\
\hline \multirow[t]{5}{*}{$\mid-131$} & 80.18 & 2.62 & $1.2392 \mathrm{E}+002$ & \multirow[t]{5}{*}{$2.5067 E+000$} & $2.8936 \mathrm{E}+001$ \\
\hline & 284.30 & 6.05 & $2.5682 E+001$ & & $7.1104 \mathrm{E}+000$ \\
\hline & 364.48 & 81.20 & $2.5067 \mathrm{E}+000$ & & $1.3263 \mathrm{E}+000$ \\
\hline & 636.97 & 7.26 & $2.3253 \mathrm{E}+001$ & & $-5.4762 E+000$ \\
\hline & 722.89 & 1.80 & $1.1684 \mathrm{E}+002$ & & $-6.0854 \mathrm{E}+000$ \\
\hline \multirow[t]{2}{*}{$\mid-133$} & 529.87 & 86.30 & $2.2709 \mathrm{E}+000$ & \multirow[t]{2}{*}{$2.2709 \mathrm{E}+000$} & 4.4043E-002 \\
\hline & 875.33 & 4.47 & $4.0428 E+001$ & & $1.0012 \mathrm{E}+001$ \\
\hline \multirow[t]{9}{*}{ CS-134 } & 475.35 & 1.46 & $1.4660 \mathrm{E}+002$ & \multirow[t]{9}{*}{$2.6834 \mathrm{E}+000$} & 5.2715E-001 \\
\hline & 563.23 & 8.38 & $2.7177 \mathrm{E}+001$ & & $-2.5456 \mathrm{E}+000$ \\
\hline & 569.32 & 15.43 & $1.2446 \mathrm{E}+001$ & & $-8.3686 \mathrm{E}-001$ \\
\hline & 604.70 & 97.60 & $2.6834 \mathrm{E}+000$ & & 7.0439E-001 \\
\hline & 795.84 & 85.40 & $3.0362 E+000$ & & 6.6194E-001 \\
\hline & 801.93 & 8.73 & $2.5997 \mathrm{E}+001$ & & $1.5044 \mathrm{E}+000$ \\
\hline & 1038.57 & 1.00 & $2.8684 \mathrm{E}+002$ & & $3.0149 \mathrm{E}+001$ \\
\hline & 1167.94 & 1.80 & $1.2329 \mathrm{E}+002$ & & $-7.6329 \mathrm{E}+001$ \\
\hline & 1365.15 & 3.04 & $8.2591 \mathrm{E}+001$ & & $-1.9175 E+001$ \\
\hline CS-137 & 661.65 & 85.12 & $2.6524 \mathrm{E}+000$ & $2.6524 \mathrm{E}+000$ & 1.8465E-001 \\
\hline \multirow[t]{4}{*}{ BA-140 } & 162.64 & 6.70 & $3.2369 E+001$ & \multirow[t]{4}{*}{$8.2184 \mathrm{E}+000$} & $-9.1150 E+000$ \\
\hline & 304.84 & 4.50 & $2.8715 E+001$ & & $-6.2411 E+000$ \\
\hline & 423.70 & 3.20 & $5.8919 E+001$ & & $-4.7762 \mathrm{E}+000$ \\
\hline & 437.55 & 2.00 & $1.2759 \mathrm{E}+002$ & & 4.6496E+001 \\
\hline
\end{tabular}




\begin{tabular}{|c|c|c|c|c|c|}
\hline & 537.32 & 25.00 & $8.2184 \mathrm{E}+000$ & & 1.0651E-001 \\
\hline \multirow[t]{7}{*}{ LA-140 } & 328.77 & 20.50 & $7.1444 \mathrm{E}+000$ & \multirow[t]{7}{*}{$1.8059 \mathrm{E}+000$} & -8.2283E-001 \\
\hline & 432.53 & 2.94 & $7.1861 \mathrm{E}+001$ & & $2.7367 \mathrm{E}+000$ \\
\hline & 487.03 & 45.50 & $4.5561 \mathrm{E}+000$ & & $2.7208 \mathrm{E}-001$ \\
\hline & 751.79 & 4.40 & $4.9212 \mathrm{E}+001$ & & $1.3385 \mathrm{E}+001$ \\
\hline & 815.85 & 23.50 & $9.2352 \mathrm{E}+000$ & & 8.0846E-001 \\
\hline & 867.82 & 5.63 & $3.4964 \mathrm{E}+001$ & & $2.3698 \mathrm{E}+000$ \\
\hline & 919.63 & 2.88 & $6.4966 \mathrm{E}+001$ & & $-4.0221 \mathrm{E}+000$ \\
\hline
\end{tabular}

User: David Georgeson

Database: InVivo Server: 127.0.0.1 
NUCLIDE MDA RESULTS FOR Accuscan II <Summed>

\begin{tabular}{|c|c|c|c|c|c|}
\hline \begin{tabular}{|l} 
Nuclide \\
Name
\end{tabular} & Energy (keV) & Yield (\%) & $\begin{array}{l}\text { Line MDA } \\
\text { (nCi) }\end{array}$ & $\begin{array}{l}\text { Nuclide MDA } \\
\text { (nCi) }\end{array}$ & Activity (nCi) \\
\hline \multirow[t]{2}{*}{ LA-140 } & 925.24 & 7.09 & $3.9042 \mathrm{E}+001$ & \multirow[t]{2}{*}{$1.8059 \mathrm{E}+000$} & $4.9222 \mathrm{E}+000$ \\
\hline & 1596.49 & 95.49 & $1.8059 \mathrm{E}+000$ & & $1.8858 \mathrm{E}-001$ \\
\hline CE-141 & 145.44 & 48.40 & $5.2862 \mathrm{E}+000$ & $5.2862 \mathrm{E}+000$ & 2.4244E-001 \\
\hline \multirow[t]{2}{*}{ CE-144 } & 80.11 & 1.60 & $2.0299 \mathrm{E}+002$ & \multirow[t]{2}{*}{$2.5596 \mathrm{E}+001$} & $4.7400 \mathrm{E}+001$ \\
\hline & 133.54 & 10.80 & $2.5596 \mathrm{E}+001$ & & $9.9842 \mathrm{E}+000$ \\
\hline \multirow[t]{11}{*}{ EU-152 } & 121.78 & 28.40 & $9.3976 \mathrm{E}+000$ & \multirow[t]{11}{*}{$6.2201 \mathrm{E}+000$} & $2.8961 \mathrm{E}+000$ \\
\hline & 244.69 & 7.49 & $2.6575 E+001$ & & $4.1789 \mathrm{E}+000$ \\
\hline & 344.27 & 26.50 & $6.2201 \mathrm{E}+000$ & & $-3.1430 \mathrm{E}+000$ \\
\hline & 411.11 & 2.21 & $8.3055 E+001$ & & $-1.2330 \mathrm{E}+001$ \\
\hline & 443.98 & 3.11 & $5.6038 \mathrm{E}+001$ & & $-2.3400 E+000$ \\
\hline & 778.89 & 12.74 & $2.1570 \mathrm{E}+001$ & & $2.2984 \mathrm{E}+000$ \\
\hline & 867.32 & 4.16 & $4.3153 E+001$ & & $-8.0149 E+000$ \\
\hline & 964.01 & 14.40 & $2.1437 E+001$ & & $4.4348 \mathrm{E}+000$ \\
\hline & 1085.78 & 10.00 & $2.9610 \mathrm{E}+001$ & & $5.2835 \mathrm{E}+000$ \\
\hline & 1112.02 & 13.30 & $1.6094 \mathrm{E}+001$ & & $1.9928 \mathrm{E}+000$ \\
\hline & 1407.95 & 20.70 & 1.2457E+001 & & $-3.8562 \mathrm{E}-001$ \\
\hline
\end{tabular}

$+=$ Nuclide Identified during the nuclide identification

$*$ = Energy Line found in the spectrum

Summed

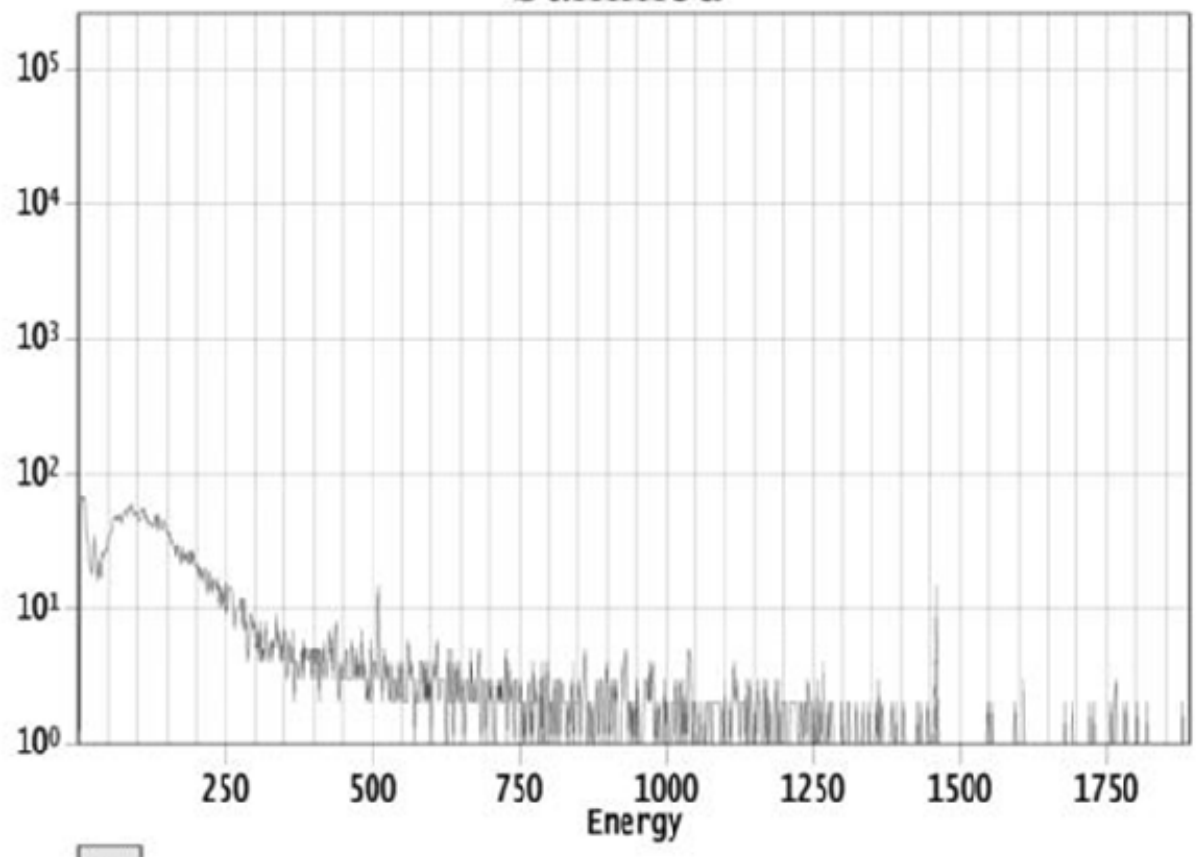

ROI Type: 1 


\section{Appendix $\mathrm{H}$}

\section{Calibration Validation Using a DOE Manufactured BOMAB \#MFP-1-001}




\title{
Appendix $\mathrm{H}$ \\ Calibration Validation Using a DOE Manufactured BOMAB \#MFP-1-001 \\ SUMMARY
}

\author{
Calibration Validation Counts \\ Accuscan II High Energy Calibration for Lung Geometry
}

Arrangement/Geometry: Fixed Detectors

Analysis Library: Am-241/Eu-152 CWI Lung Set

Energy Calibration Date: 4/5/2011 8:18

Efficiency Calibration Date: 4/5/2011 4:05

Live Time: 600 seconds

Count Start Times:

Count \# 1 - 4/5/2011 4:18 PM

Count \# 2 - 4/6/2011 8:24

Count \# 3 - 4/6/2011 8:47

Count \# 4 - 4/6/2011 9:13

Count \# 5 - 4/6/2011 9:35

\begin{tabular}{ccccccccc}
$\begin{array}{c}\text { Nuclide/ } \\
\text { Energy } \\
\text { (keV) }\end{array}$ & $\begin{array}{c}\text { Expected } \\
\text { Activity } \\
\text { (nCi) }\end{array}$ & $\# 1$ & $\# 2$ & $\# 3$ & $\# 4$ & $\# 5$ & $\begin{array}{c}\text { Relative } \\
\text { Bias }\end{array}$ & $\begin{array}{c}\text { Relative } \\
\text { Precision }\end{array}$ \\
\hline Co-60 & 161.0000 & 162.53 & 162.91 & 162.86 & 160.15 & 159.3 & 0.003 & 0.011 \\
Cs-137 & 398.0000 & 407.9 & 392.6 & 397.97 & 382.6 & 391.51 & -0.009 & 0.023 \\
Cs-134 & 53.2000 & 51.33 & 52.73 & 53.83 & 48.64 & 50.44 & -0.034 & 0.038 \\
Co-57 & 16.9000 & 11.690 & 19.140 & 16.530 & 15.220 & 18.880 & -0.036 & 0.180 \\
Mn-54 & 4.4000 & & & & & & &
\end{tabular}

Average $\quad-0.036 \quad 0.180$




\section{COUNT 1}

\section{Apex-InVivo}

\section{Analysis Report - Lung Test [999-99-9996]}

\section{SOURCE INFORMATION}

Primary ID

Last Name

First Name

Height (in)

Weight (lb)

Chest Wall Thickness (cm)
999-99-9996

Test

Lung

\section{COUNTER INFORMATION}

Counter Name

Counter Location

Configuration Name
Accuscan II

Battelle

Lung - High Energy

\section{COUNT INFORMATION}

\begin{tabular}{ll}
$\begin{array}{l}\text { Count Operation } \\
\text { Count Reason } \\
\text { Frequency } \\
\text { Comment }\end{array}$ & $\begin{array}{l}\text { Individual } 5 \text { minutes } \\
\text { Routine operation }\end{array}$ \\
Intake Date & \\
Acquisition Started & \\
Acquisition Time & $4 / 5 / 2011$ 11:00:51 AM \\
Operator Name & 301.21 \\
Count Status & David Georgeson \\
Primary Review & Not Reviewed \\
Secondary Review & No \\
\hline
\end{tabular}

DETECTOR COUNT RATE REPORT

\begin{tabular}{|l|r|r|r|c|}
\hline \multicolumn{1}{|c|}{ Detector Name } & Count Rate & $\begin{array}{c}\text { Count Rate } \\
\text { Range }\end{array}$ & Live Time & Analyzed \\
\hline Right & 84.73 & $1-4096$ & 300.0 & No \\
\hline Left & 81.53 & $1-4096$ & 300.0 & No \\
\hline Summed & 166.31 & $1-4096$ & 300.0 & Yes \\
\hline
\end{tabular}

Primary Review by:

Date:

User: David Georgeson 


\section{DETECTOR GROUP INFORMATION}

$\begin{array}{ll}\text { Detector Group Name } & \text { Summed } \\ \text { Detector Group Configuration } & \text { Lung Screening } \\ \text { Analysis Date } & \text { 4/5/2011 11:00:42 AM } \\ \text { Energy Calibration } & \text { Energy Calibration (HE Lung) 4/5/2011 8:18 AM } \\ \text { Efficiency Calibration } & \text { HE Lung Realistic Summed Eff Cal 4/5/2011 10:43 AM (Dual) } \\ \quad \text { Multi Curve } & \text { No } \\ \text { Source Distribution } & \text { Lung } \\ \text { Analysis Sequence File } & \text { ROUNPPGE.ASF } \\ \text { Bkg Acq used for area correction } & \text { Not Performed } \\ \text { Nuclide Identification } & \\ \quad \text { Nuclide Identification Library } & \text { INLPLANTLIB.NLB } \\ \quad \text { Tentative NID Library } & \text { INLPLANTLIB.NLB } \\ \quad \text { Peak Search Library } & \text { INLPLANTLIB.NLB } \\ \quad \text { Analysis Limits (channels) } & \text { 40 - 4095 } \\ \text { Energy Tolerance (FWHM) } & \text { 1.20 } \\ \text { Comment } & \end{array}$

PEAK SEARCH RESULTS FOR Accuscan II <Summed>

\begin{tabular}{|r|r|r|r|r|r||}
\hline \multicolumn{7}{|c|}{ Peak Analysis } \\
\hline & \multicolumn{1}{|l|}{ No } & $\begin{array}{l}\text { leak } \\
\text { Centroid }\end{array}$ & \multicolumn{1}{l}{$\begin{array}{l}\text { Energy } \\
\text { (keV) }\end{array}$} & $\begin{array}{l}\text { Net Peak } \\
\text { Area }\end{array}$ & $\begin{array}{l}\text { Continuum } \\
\text { Counts }\end{array}$ \\
\hline & 1 & 69.76 & 32.12 & $1.56 \mathrm{E}+002$ & $3.30 \mathrm{E}+002$ \\
\hline & 2 & 264.85 & 122.17 & $2.77 \mathrm{E}+002$ & $6.90 \mathrm{E}+002$ \\
\hline & 3 & 1309.67 & 604.65 & $3.04 \mathrm{E}+002$ & $8.62 \mathrm{E}+001$ \\
\hline & 4 & 1432.89 & 661.57 & $1.87 \mathrm{E}+003$ & $6.63 \mathrm{E}+001$ \\
\hline & 5 & 1723.32 & 795.75 & $2.04 \mathrm{E}+002$ & $4.92 \mathrm{E}+001$ \\
\hline & 6 & 2540.05 & 1173.20 & $5.28 \mathrm{E}+002$ & $6.69 \mathrm{E}+001$ \\
\hline 7 & 2884.51 & 1332.45 & $5.49 \mathrm{E}+002$ & $4.27 \mathrm{E}+000$ \\
\hline 8 & 3162.18 & 1460.85 & $2.55 \mathrm{E}+001$ & $2.48 \mathrm{E}+000$ \\
\hline
\end{tabular}

For unknown peaks we assume yield to be $100 \%$ and no decay correction is performed

$M=$ First peak in a multiplet region

$\mathrm{m}=$ Other peak in a multiplet region

$\mathrm{F}=$ Fitted singlet

NUCLIDE RESULTS FOR Accuscan II <Summed>

\begin{tabular}{|l|l|r|r|r|}
\hline & $\begin{array}{l}\text { Nuclide } \\
\text { Name }\end{array}$ & $\begin{array}{l}\text { Id } \\
\text { Confidence }\end{array}$ & $\begin{array}{l}\text { Wt Mean Activity } \\
\text { (nCi) }\end{array}$ & Error (1SD) \\
\hline K-40 & 1.000 & $1.619072 \mathrm{E}+002$ & $23.13 \%$ \\
\hline CO-57 & 1.000 & $3.872478 \mathrm{E}+001$ & $23.24 \%$ \\
\hline CO-60 & 1.000 & $3.387544 \mathrm{E}+002$ & $3.55 \%$ \\
\hline CS-134 & 0.880 & $1.063828 \mathrm{E}+002$ & $6.67 \%$ \\
\hline & CS-137 & 1.000 & $8.208113 \mathrm{E}+002$ & $2.72 \%$ \\
\hline
\end{tabular}

$?=$ Nuclide is part of an undetermined solution

$\mathrm{X}=$ Nuclide rejected by the interference analysis

$@$ @ = Nuclide contains energy lines not used in Weighted Mean Activity

! = Nuclide was corrected for parent/daughter 
NUCLIDE MDA RESULTS FOR Accuscan II <Summed>

\begin{tabular}{|r|l|r|r|l|}
\hline & $\begin{array}{l}\text { Nuclide } \\
\text { Name }\end{array}$ & Energy (keV) & Yield (\%) & $\begin{array}{l}\text { Line MDA } \\
\text { (nCi) }\end{array}$ \\
\hline+ & K-40 & $1460.81 *$ & 10.67 & $1.0766 \mathrm{E}+002$ \\
\hline \multirow{2}{*}{} & CR-51 & 320.08 & 9.83 & $1.5974 \mathrm{E}+002$ \\
\hline \multirow{2}{*}{} & MN-54 & 834.83 & 99.97 & $1.7832 \mathrm{E}+001$ \\
\hline+ & CO-57 & $122.06 *$ & 85.51 & $4.0816 \mathrm{E}+001$ \\
\cline { 1 - 4 }+ & & 136.48 & 10.60 & $1.5067 \mathrm{E}+002$ \\
\hline
\end{tabular}

User: David Georgeson 
Analysis Report - Lung Test [999-99-9996]

\section{NUCLIDE MDA RESULTS FOR Accuscan II <Summed>}

\begin{tabular}{|c|c|c|c|c|}
\hline & \begin{tabular}{|l|} 
Nuclide \\
Name
\end{tabular} & Energy (keV) & Yield (\%) & Line MDA (nCi) \\
\hline & CO-58 & 810.76 & 99.40 & $1.5478 \mathrm{E}+001$ \\
\hline & \multirow[t]{4}{*}{ FE-59 } & 142.65 & 1.03 & $1.4739 \mathrm{E}+003$ \\
\hline & & 192.34 & 3.11 & $5.6446 \mathrm{E}+002$ \\
\hline & & 1099.22 & 56.50 & $2.7983 \mathrm{E}+001$ \\
\hline & & 1291.56 & 43.20 & $1.4145 \mathrm{E}+001$ \\
\hline+ & \multirow[t]{2}{*}{ CO-60 } & 1173.22 * & 100.00 & $5.3892 \mathrm{E}+001$ \\
\hline+ & & 1332.49 * & 100.00 & $1.6286 \mathrm{E}+001$ \\
\hline & ZN-65 & 1115.52 & 50.75 & $3.7810 \mathrm{E}+001$ \\
\hline & NB-95 & 765.79 & 99.81 & $1.5238 \mathrm{E}+001$ \\
\hline & \multirow[t]{2}{*}{ ZR-95 } & 724.18 & 43.70 & $3.2542 \mathrm{E}+001$ \\
\hline & & 756.72 & 55.30 & $2.0945 \mathrm{E}+001$ \\
\hline & \multirow[t]{2}{*}{ RU-106 } & 621.84 & 9.80 & $1.5947 \mathrm{E}+002$ \\
\hline & & 1050.47 & 1.73 & $7.9329 \mathrm{E}+002$ \\
\hline & \multirow[t]{15}{*}{ AG-110M } & 446.80 & 3.64 & $5.1419 \mathrm{E}+002$ \\
\hline & & 620.35 & 2.77 & $5.5632 \mathrm{E}+002$ \\
\hline & & 657.75 & 94.40 & $1.8557 \mathrm{E}+001$ \\
\hline & & 677.61 & 10.68 & 1.1309E+002 \\
\hline & & 686.99 & 6.47 & $2.2170 \mathrm{E}+002$ \\
\hline & & 706.67 & 16.68 & $9.1072 \mathrm{E}+001$ \\
\hline & & 744.26 & 4.64 & $3.1978 \mathrm{E}+002$ \\
\hline & & 763.93 & 22.28 & $6.0116 \mathrm{E}+001$ \\
\hline & & 818.02 & 7.30 & $2.0812 \mathrm{E}+002$ \\
\hline & & 884.67 & 72.60 & $2.0945 \mathrm{E}+001$ \\
\hline & & 937.48 & 34.20 & $5.3882 E+001$ \\
\hline & & 1384.27 & 24.26 & $3.3547 \mathrm{E}+001$ \\
\hline & & 1475.76 & 3.97 & $2.1055 \mathrm{E}+002$ \\
\hline & & 1505.00 & 13.06 & $1.5712 \mathrm{E}+001$ \\
\hline & & 1562.27 & 1.18 & $8.4290 \mathrm{E}+002$ \\
\hline & \multirow[t]{5}{*}{ I-131 } & 80.18 & 2.62 & $4.4673 \mathrm{E}+002$ \\
\hline & & 284.30 & 6.05 & $2.4532 \mathrm{E}+002$ \\
\hline & & 364.48 & 81.20 & $2.1755 \mathrm{E}+001$ \\
\hline & & 636.97 & 7.26 & $2.2738 \mathrm{E}+002$ \\
\hline & & 722.89 & 1.80 & $7.0932 \mathrm{E}+002$ \\
\hline & \multirow[t]{2}{*}{ I-133 } & 529.87 & 86.30 & $1.7857 \mathrm{E}+001$ \\
\hline & & 875.33 & 4.47 & $2.8436 \mathrm{E}+002$ \\
\hline+ & \multirow[t]{3}{*}{ CS-134 } & 475.35 & 1.46 & 1.4419E+003 \\
\hline+ & & 563.23 & 8.38 & $2.2787 \mathrm{E}+002$ \\
\hline+ & & 569.32 & 15.43 & $1.4267 \mathrm{E}+002$ \\
\hline
\end{tabular}




\begin{tabular}{|c|c|c|c|c|}
\hline+ & & 604.70 * & 97.60 & $3.4462 \mathrm{E}+001$ \\
\hline+ & & 795.84 * & 85.40 & $3.6731 \mathrm{E}+001$ \\
\hline+ & & 801.93 & 8.73 & $2.3311 \mathrm{E}+002$ \\
\hline+ & & 1038.57 & 1.00 & $1.8555 \mathrm{E}+003$ \\
\hline+ & & 1167.94 & 1.80 & $1.2143 E+003$ \\
\hline+ & & 1365.15 & 3.04 & $5.5737 \mathrm{E}+002$ \\
\hline+ & CS-137 & 661.65 * & 85.12 & $3.6557 \mathrm{E}+001$ \\
\hline & BA-140 & 162.64 & 6.70 & $2.4230 \mathrm{E}+002$ \\
\hline
\end{tabular}

User: David Georgeson 
Analysis Report - Lung Test [999-99-9996]

\section{NUCLIDE MDA RESULTS FOR Accuscan II <Summed>}

\begin{tabular}{|c|c|c|c|}
\hline Nuclide & Energy (keV) & Yield (\%) & Line MDA \\
\hline \multirow[t]{4}{*}{ BA-140 } & 304.84 & 4.50 & $3.2793 \mathrm{E}+002$ \\
\hline & 423.70 & 3.20 & $6.1478 E+002$ \\
\hline & 437.55 & 2.00 & $8.9001 \mathrm{E}+002$ \\
\hline & 537.32 & 25.00 & $6.8449 \mathrm{E}+001$ \\
\hline \multirow[t]{9}{*}{ LA-140 } & 328.77 & 20.50 & $7.2270 \mathrm{E}+001$ \\
\hline & 432.53 & 2.94 & $6.4535 E+002$ \\
\hline & 487.03 & 45.50 & $3.6939 \mathrm{E}+001$ \\
\hline & 751.79 & 4.40 & $3.6418 \mathrm{E}+002$ \\
\hline & 815.85 & 23.50 & $5.7389 \mathrm{E}+001$ \\
\hline & 867.82 & 5.63 & $3.4215 \mathrm{E}+002$ \\
\hline & 919.63 & 2.88 & $7.4100 \mathrm{E}+002$ \\
\hline & 925.24 & 7.09 & $3.0255 \mathrm{E}+002$ \\
\hline & 1596.49 & 95.49 & $8.9436 \mathrm{E}+000$ \\
\hline CE-141 & 145.44 & 48.40 & $3.4561 \mathrm{E}+001$ \\
\hline \multirow[t]{2}{*}{ CE-144 } & 80.11 & 1.60 & $7.3105 \mathrm{E}+002$ \\
\hline & 133.54 & 10.80 & $1.4124 \mathrm{E}+002$ \\
\hline \multirow[t]{11}{*}{ EU-152 } & 121.78 & 28.40 & $7.2607 \mathrm{E}+001$ \\
\hline & 244.69 & 7.49 & $2.2678 \mathrm{E}+002$ \\
\hline & 344.27 & 26.50 & $6.0718 \mathrm{E}+001$ \\
\hline & 411.11 & 2.21 & $8.8878 E+002$ \\
\hline & 443.98 & 3.11 & $6.1895 E+002$ \\
\hline & 778.89 & 12.74 & $1.0943 \mathrm{E}+002$ \\
\hline & 867.32 & 4.16 & $4.8187 \mathrm{E}+002$ \\
\hline & 964.01 & 14.40 & $1.2863 E+002$ \\
\hline & 1085.78 & 10.00 & $1.5157 \mathrm{E}+002$ \\
\hline & 1112.02 & 13.30 & $1.1601 \mathrm{E}+002$ \\
\hline & 1407.95 & 20.70 & $4.6360 \mathrm{E}+001$ \\
\hline
\end{tabular}

$+=$ Nuclide Identified during the nuclide identification

$*$ = Energy Line found in the spectrum 


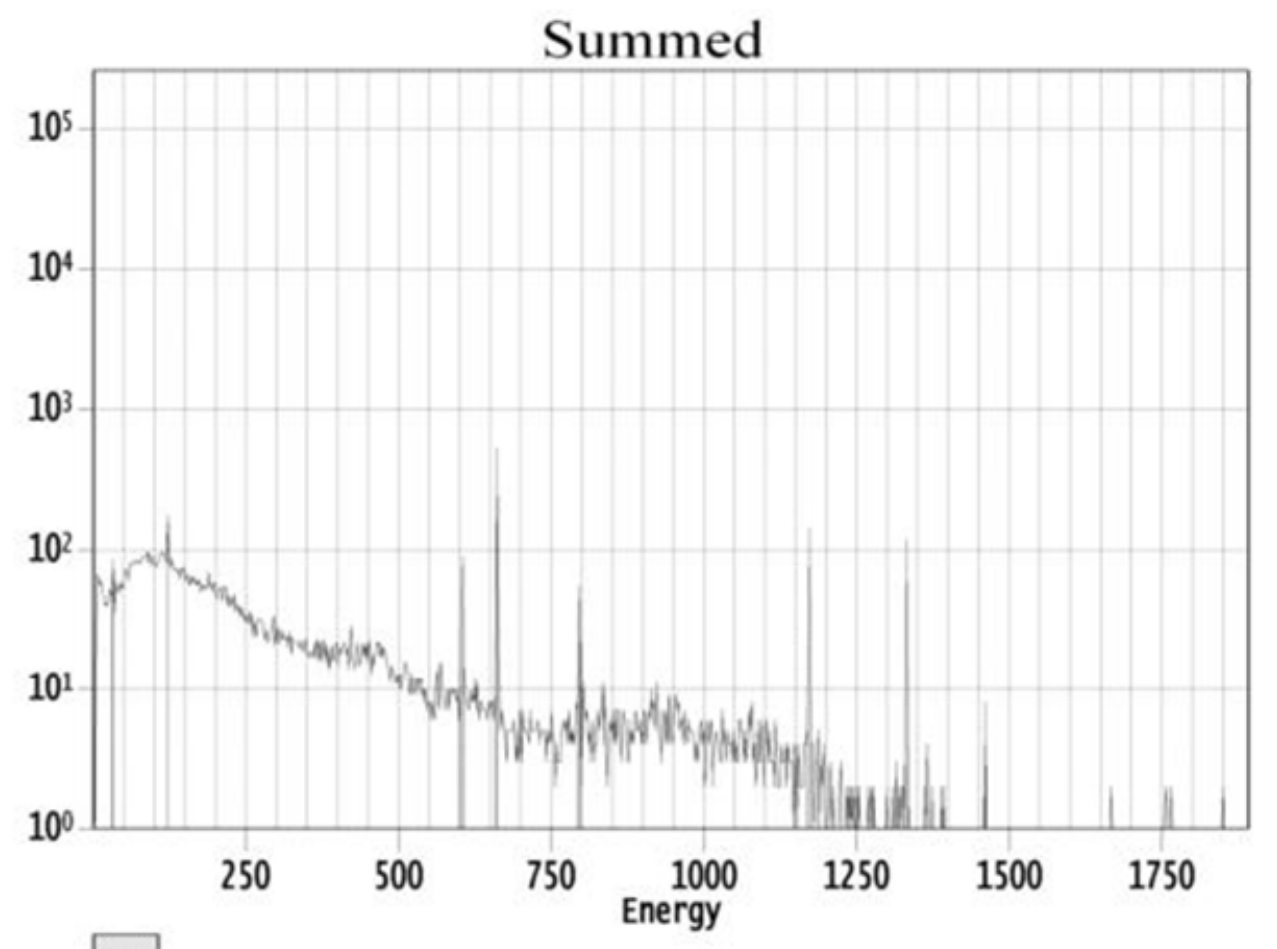

ROI Type: 1 


\section{COUNT 2}

\section{Apex-InVivo}

\section{Analysis Report - Lung Test [999-99-9996]}

\section{SOURCE INFORMATION}

$\begin{array}{ll}\text { Primary ID } & 999-99-9996 \\ \text { Last Name } & \text { Test } \\ \text { First Name } & \text { Lung } \\ \text { Height (in) } & \\ \text { Weight (lb) } & \\ \text { Chest Wall Thickness (cm) } & \end{array}$

\section{COUNTER INFORMATION}

Counter Name
Counter Location
Configuration Name

\section{COUNT INFORMATION}

Count Operation

Count Reason

Frequency

Comment

Intake Date

Acquisition Started

Acquisition Time

Operator Name

Count Status

Primary Review

Secondary Review
Accuscan II

Battelle

Lung - High Energy

Individual 10 minutes

Routine operation

MFP-1-001 Validation Count 2

4/6/2011 8:24:09 AM

602.21

David Georgeson

Not Reviewed

No

No

\section{DETECTOR COUNT RATE REPORT}

\begin{tabular}{|l|r|r|r|c|}
\hline \multicolumn{1}{|c|}{ Detector Name } & $\begin{array}{c}\text { Count } \\
\text { Rate }\end{array}$ & $\begin{array}{c}\text { Count Rate } \\
\text { Range }\end{array}$ & Live Time & Analyzed \\
\hline Left & 82.01 & $1-4096$ & 600.0 & No \\
\hline Right & 83.62 & $1-4096$ & 600.0 & No \\
\hline Summed & 165.08 & $1-4096$ & 600.0 & Yes \\
\hline
\end{tabular}

Primary Review by:

Date:

User: David Georgeson 


\section{DETECTOR GROUP INFORMATION}

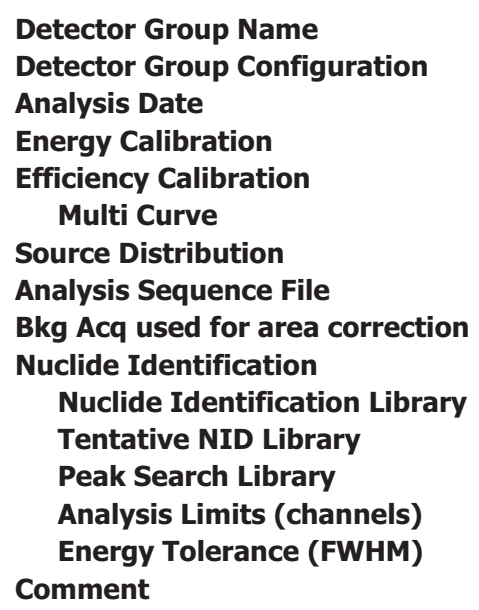

PEAK SEARCH RESULTS FOR Accuscan II <Summed>

\begin{tabular}{|r|r|r|r|r|r||}
\hline \multicolumn{7}{|c|}{ Peak Analysis } \\
\hline & & $\begin{array}{l}\text { Peak } \\
\text { Centroid }\end{array}$ & $\begin{array}{l}\text { Energy } \\
\text { (keV) }\end{array}$ & \multicolumn{1}{l||}{ Net Peak Area } & $\begin{array}{l}\text { Continuum } \\
\text { Counts }\end{array}$ \\
\hline & 1 & 69.84 & 32.15 & $1.64 \mathrm{E}+002$ & $8.35 \mathrm{E}+002$ \\
\hline M & 2 & 264.74 & 122.12 & $5.77 \mathrm{E}+002$ & $1.52 \mathrm{E}+003$ \\
\hline $\mathrm{m}$ & 3 & 1220.16 & 563.30 & $6.12 \mathrm{E}+001$ & $1.34 \mathrm{E}+002$ \\
\hline & 5 & 1232.70 & 569.09 & $1.14 \mathrm{E}+002$ & $1.90 \mathrm{E}+002$ \\
\hline & 6 & 1309.73 & 604.67 & $6.15 \mathrm{E}+002$ & $1.65 \mathrm{E}+002$ \\
\hline $\mathrm{M}$ & 7 & 1723.48 & 795.82 & $4.25 \mathrm{E}+002$ & $8.97 \mathrm{E}+001$ \\
\hline $\mathrm{m}$ & 8 & 1736.87 & 802.01 & $2.77 \mathrm{E}+001$ & $1.10 \mathrm{E}+002$ \\
\hline & 9 & 2540.08 & 1173.22 & $1.16 \mathrm{E}+003$ & $5.40 \mathrm{E}+001$ \\
\hline & 10 & 2884.62 & 1332.50 & $1.09 \mathrm{E}+003$ & $1.27 \mathrm{E}+001$ \\
\hline & 11 & 3161.97 & 1460.75 & $6.55 \mathrm{E}+001$ & $2.52 \mathrm{E}+000$ \\
\hline
\end{tabular}

For unknown peaks we assume yield to be $100 \%$ and no decay correction is performed

$M=$ First peak in a multiplet region

$\mathrm{m}=$ Other peak in a multiplet region

$\mathrm{F}=$ Fitted singlet

NUCLIDE RESULTS FOR Accuscan II <Summed>

\begin{tabular}{|l|l|r|r|r|}
\hline & $\begin{array}{l}\text { Nuclide } \\
\text { Name }\end{array}$ & $\begin{array}{l}\text { Id } \\
\text { Confidence }\end{array}$ & $\begin{array}{l}\text { Wt Mean } \\
\text { Activity (nCi) }\end{array}$ & Error (1SD) \\
\hline K-40 & 1.000 & $1.012900 \mathrm{E}+00$ & $13.36 \%$ \\
\hline CO-57 & 1.000 & $1.914159 \mathrm{E}+00$ & $17.75 \%$ \\
\hline CO-60 & 1.000 & $1.629071 \mathrm{E}+00$ & $2.39 \%$ \\
\hline CS-134 & 1.000 & $5.272515 \mathrm{E}+00$ & $3.66 \%$ \\
\hline CS-137 & 1.000 & $3.925997 \mathrm{E}+00$ & $2.07 \%$ \\
\hline
\end{tabular}

$?=$ Nuclide is part of an undetermined solution

$\mathrm{X}=$ Nuclide rejected by the interference analysis

$@$ @ = Nuclide contains energy lines not used in Weighted Mean Activity

! = Nuclide was corrected for parent/daughter
Summed

Energy Calibration (HE Lung) 4/5/2011 8:18 AM

Calibration (HE Lung Realistic)4/5/2011 4:05 PM (Dual)

Lung

INLPLANTLIB.NLB

$-4095$

1.20 
NUCLIDE MDA RESULTS FOR Accuscan II <Summed>

\begin{tabular}{|c|l|r|r|r|}
\hline & $\begin{array}{l}\text { Nuclide } \\
\text { Name }\end{array}$ & Energy (keV) & Yield (\%) & Line MDA (nCi) \\
\hline$+>$ & K-40 & $1460.81 *$ & 10.67 & $0.0000 \mathrm{E}+000$ \\
\hline$>$ & CR-51 & 320.08 & 9.83 & $0.0000 \mathrm{E}+000$ \\
\hline
\end{tabular}

User: David Georgeson 
Analysis Report - Lung Test [999-99-9996]

NUCLIDE MDA RESULTS FOR Accuscan II <Summed>

\begin{tabular}{|c|c|c|c|c|}
\hline & \begin{tabular}{|l|} 
Nuclide \\
Name
\end{tabular} & Energy (keV) & Yield (\%) & Line MDA (nCi) \\
\hline$>$ & MN-54 & 834.83 & 99.97 & $0.0000 \mathrm{E}+000$ \\
\hline$+>$ & \multirow[t]{2}{*}{ CO-57 } & $122.06 *$ & 85.51 & $0.0000 \mathrm{E}+000$ \\
\hline$+>$ & & 136.48 & 10.60 & $0.0000 \mathrm{E}+000$ \\
\hline$>$ & CO-58 & 810.76 & 99.40 & $0.0000 \mathrm{E}+000$ \\
\hline$>$ & \multirow[t]{4}{*}{ FE-59 } & 142.65 & 1.03 & $0.0000 \mathrm{E}+000$ \\
\hline$>$ & & 192.34 & 3.11 & $0.0000 \mathrm{E}+000$ \\
\hline$>$ & & 1099.22 & 56.50 & $0.0000 \mathrm{E}+000$ \\
\hline$>$ & & 1291.56 & 43.20 & $0.0000 \mathrm{E}+000$ \\
\hline$+>$ & \multirow[t]{2}{*}{ CO-60 } & $1173.22 *$ & 100.00 & $0.0000 \mathrm{E}+000$ \\
\hline$+>$ & & $1332.49 *$ & 100.00 & $0.0000 \mathrm{E}+000$ \\
\hline$>$ & ZN-65 & 1115.52 & 50.75 & $0.0000 \mathrm{E}+000$ \\
\hline$>$ & NB-95 & 765.79 & 99.81 & $0.0000 E+000$ \\
\hline$>$ & \multirow[t]{2}{*}{ ZR-95 } & 724.18 & 43.70 & $0.0000 \mathrm{E}+000$ \\
\hline$>$ & & 756.72 & 55.30 & $0.0000 \mathrm{E}+000$ \\
\hline$>$ & \multirow[t]{2}{*}{$R U-106$} & 621.84 & 9.80 & $0.0000 E+000$ \\
\hline$>$ & & 1050.47 & 1.73 & $0.0000 \mathrm{E}+000$ \\
\hline$>$ & \multirow[t]{15}{*}{ AG-110M } & 446.80 & 3.64 & $0.0000 \mathrm{E}+000$ \\
\hline$>$ & & 620.35 & 2.77 & $0.0000 E+000$ \\
\hline$>$ & & 657.75 & 94.40 & $0.0000 \mathrm{E}+000$ \\
\hline$>$ & & 677.61 & 10.68 & $0.0000 \mathrm{E}+000$ \\
\hline$>$ & & 686.99 & 6.47 & $0.0000 \mathrm{E}+000$ \\
\hline$>$ & & 706.67 & 16.68 & $0.0000 \mathrm{E}+000$ \\
\hline$>$ & & 744.26 & 4.64 & $0.0000 \mathrm{E}+000$ \\
\hline$>$ & & 763.93 & 22.28 & $0.0000 \mathrm{E}+000$ \\
\hline$>$ & & 818.02 & 7.30 & $0.0000 \mathrm{E}+000$ \\
\hline$>$ & & 884.67 & 72.60 & $0.0000 \mathrm{E}+000$ \\
\hline$>$ & & 937.48 & 34.20 & $0.0000 \mathrm{E}+000$ \\
\hline$>$ & & 1384.27 & 24.26 & $0.0000 \mathrm{E}+000$ \\
\hline$>$ & & 1475.76 & 3.97 & $0.0000 \mathrm{E}+000$ \\
\hline$>$ & & 1505.00 & 13.06 & $0.0000 \mathrm{E}+000$ \\
\hline$>$ & & 1562.27 & 1.18 & $0.0000 E+000$ \\
\hline$>$ & \multirow[t]{5}{*}{$\mid-131$} & 80.18 & 2.62 & $0.0000 E+000$ \\
\hline$>$ & & 284.30 & 6.05 & $0.0000 \mathrm{E}+000$ \\
\hline$>$ & & 364.48 & 81.20 & $0.0000 \mathrm{E}+000$ \\
\hline$>$ & & 636.97 & 7.26 & $0.0000 \mathrm{E}+000$ \\
\hline$>$ & & 722.89 & 1.80 & $0.0000 E+000$ \\
\hline$>$ & \multirow[t]{2}{*}{$\mid-133$} & 529.87 & 86.30 & $0.0000 \mathrm{E}+000$ \\
\hline$>$ & & 875.33 & 4.47 & $0.0000 \mathrm{E}+000$ \\
\hline
\end{tabular}




\begin{tabular}{|c|c|c|c|}
\hline \begin{tabular}{l|l}
$+>$ & CS-134
\end{tabular} & 475.35 & 1.46 & $0.0000 \mathrm{E}+000$ \\
\hline$+>$ & 563.23 * & 8.38 & $0.0000 \mathrm{E}+000$ \\
\hline$+>$ & 569.32 * & 15.43 & $0.0000 \mathrm{E}+000$ \\
\hline$+>$ & 604.70 * & 97.60 & $0.0000 \mathrm{E}+000$ \\
\hline$+>$ & 795.84 * & 85.40 & $0.0000 \mathrm{E}+000$ \\
\hline$+>$ & 801.93 * & 8.73 & $0.0000 \mathrm{E}+000$ \\
\hline$+>$ & 1038.57 & 1.00 & $0.0000 \mathrm{E}+000$ \\
\hline$+>$ & 1167.94 & 1.80 & $0.0000 \mathrm{E}+000$ \\
\hline
\end{tabular}

User: David Georgeson 
Analysis Report - Lung Test [999-99-9996]

\section{NUCLIDE MDA RESULTS FOR Accuscan II <Summed>}

\begin{tabular}{|c|c|c|c|c|}
\hline & Nuclide Name & Energy (keV) & Yield (\%) & Line MDA (nCi) \\
\hline$+>$ & CS-134 & 1365.15 & 3.04 & $0.0000 \mathrm{E}+000$ \\
\hline$+>$ & CS-137 & $661.65 *$ & 85.12 & $0.0000 E+000$ \\
\hline$>$ & BA-140 & 162.64 & 6.70 & $0.0000 \mathrm{E}+000$ \\
\hline$>$ & & 304.84 & 4.50 & $0.0000 \mathrm{E}+000$ \\
\hline$>$ & & 423.70 & 3.20 & $0.0000 \mathrm{E}+000$ \\
\hline$>$ & & 437.55 & 2.00 & $0.0000 \mathrm{E}+000$ \\
\hline$>$ & & 537.32 & 25.00 & $0.0000 \mathrm{E}+000$ \\
\hline$>$ & LA-140 & 328.77 & 20.50 & $0.0000 \mathrm{E}+000$ \\
\hline$>$ & & 432.53 & 2.94 & $0.0000 \mathrm{E}+000$ \\
\hline$>$ & & 487.03 & 45.50 & $0.0000 \mathrm{E}+000$ \\
\hline$>$ & & 751.79 & 4.40 & $0.0000 \mathrm{E}+000$ \\
\hline$>$ & & 815.85 & 23.50 & $0.0000 \mathrm{E}+000$ \\
\hline$>$ & & 867.82 & 5.63 & $0.0000 \mathrm{E}+000$ \\
\hline$>$ & & 919.63 & 2.88 & $0.0000 \mathrm{E}+000$ \\
\hline$>$ & & 925.24 & 7.09 & $0.0000 E+000$ \\
\hline$>$ & & 1596.49 & 95.49 & $0.0000 \mathrm{E}+000$ \\
\hline$>$ & CE-141 & 145.44 & 48.40 & $0.0000 E+000$ \\
\hline$>$ & CE-144 & 80.11 & 1.60 & $0.0000 \mathrm{E}+000$ \\
\hline$>$ & & 133.54 & 10.80 & $0.0000 \mathrm{E}+000$ \\
\hline$>$ & EU-152 & 121.78 & 28.40 & $0.0000 E+000$ \\
\hline$>$ & & 244.69 & 7.49 & $0.0000 \mathrm{E}+000$ \\
\hline$>$ & & 344.27 & 26.50 & $0.0000 \mathrm{E}+000$ \\
\hline$>$ & & 411.11 & 2.21 & $0.0000 E+000$ \\
\hline$>$ & & 443.98 & 3.11 & $0.0000 E+000$ \\
\hline$>$ & & 778.89 & 12.74 & $0.0000 \mathrm{E}+000$ \\
\hline$>$ & & 867.32 & 4.16 & $0.0000 \mathrm{E}+000$ \\
\hline$>$ & & 964.01 & 14.40 & $0.0000 \mathrm{E}+000$ \\
\hline$>$ & & 1085.78 & 10.00 & $0.0000 \mathrm{E}+000$ \\
\hline$>$ & & 1112.02 & 13.30 & $0.0000 \mathrm{E}+000$ \\
\hline$>$ & & 1407.95 & 20.70 & $0.0000 \mathrm{E}+000$ \\
\hline
\end{tabular}

$+=$ Nuclide Identified during the nuclide identification

$*$ = Energy Line found in the spectrum

$>=$ MDA Value not calculated 
Analysis Report - Lung Test [999-99-9996]

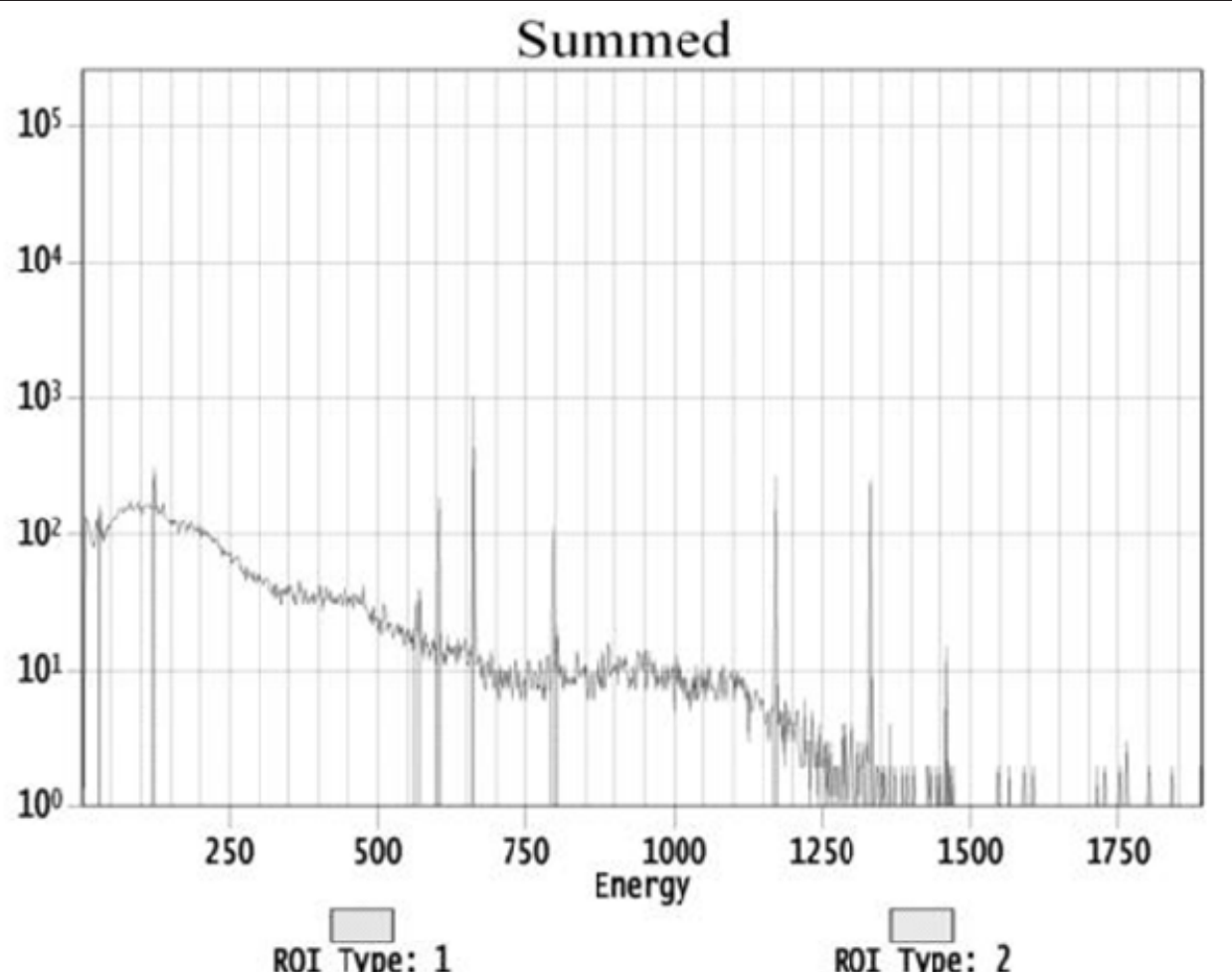

ROI Type: 1

ROI Type: 2

User: David Georgeson 


\section{COUNT 3}

\section{Apex-InVivo}

\section{Analysis Report - Lung Test [999-99-9996]}

\section{SOURCE INFORMATION}

Primary ID

Last Name

First Name

Height (in)

Weight (lb)

Chest Wall Thickness (cm)
999-99-9996

Test

Lung

\section{COUNTER INFORMATION}

Counter Name

Counter Location

Configuration Name
Accuscan II

Battelle

Lung - High Energy

\section{COUNT INFORMATION}

Count Operation

Count Reason

Frequency

Comment

Intake Date

Acquisition Started

Acquisition Time

Operator Name

Count Status

Primary Review

Secondary Review
Individual 10 minutes

Routine operation

MFP-1-001 validation count 3

4/6/2011 8:47:28 AM

602.21

David Georgeson

Not Reviewed

No

No

DETECTOR COUNT RATE REPORT

\begin{tabular}{|l|r|c|r|c|}
\hline \multicolumn{1}{|c|}{ Detector Name } & $\begin{array}{c}\text { Count } \\
\text { Rate }\end{array}$ & $\begin{array}{c}\text { Count } \\
\text { Rate }\end{array}$ & Live Time & Analyzed \\
\hline Left & 80.44 & $1-4096$ & 600.0 & No \\
\hline Right & 83.32 & $1-4096$ & 600.0 & No \\
\hline Summed & 163.23 & $1-4096$ & 600.0 & Yes \\
\hline
\end{tabular}

Primary Review by:

Date: 


\section{DETECTOR GROUP INFORMATION}

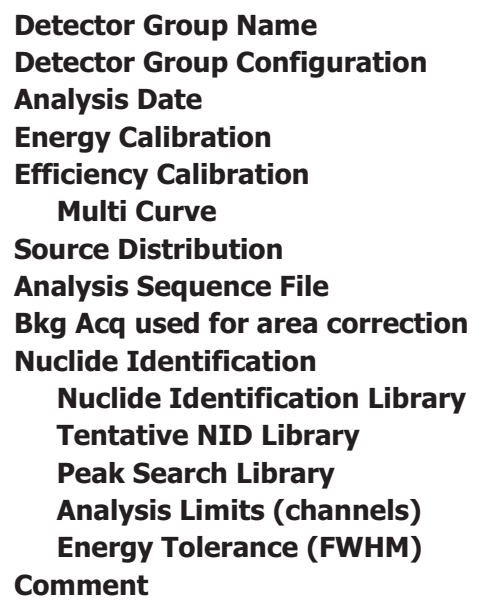

Summed

Lung Screening

4/6/2011 8:47:08 AM

Energy Calibration (HE Lung) 4/5/2011 8:18 AM

Eff. Calibration (HE Lung Realistic)4/5/2011 4:05 PM (Dual)

No

Lung

ROUNPPGE.ASF

Not Performed

INLPLANTLIB.NLB

INLPLANTLIB.NLB

INLPLANTLIB.NLB

$40-4095$

1.20

\section{PEAK SEARCH RESULTS FOR Accuscan II <Summed>}

\begin{tabular}{|r|r|r|r|r|r||}
\hline \multicolumn{7}{|c||}{ Peak Analysis } \\
\hline & \multicolumn{1}{|l|}{ No } & $\begin{array}{l}\text { Peak } \\
\text { Centroid }\end{array}$ & $\begin{array}{l}\text { Energy } \\
\text { (keV) }\end{array}$ & $\begin{array}{l}\text { Net Peak } \\
\text { Area }\end{array}$ & $\begin{array}{l}\text { Continuum } \\
\text { Counts }\end{array}$ \\
\hline & 1 & 69.80 & 32.13 & $2.91 \mathrm{E}+002$ & $7.27 \mathrm{E}+002$ \\
\hline & 2 & 264.54 & 122.03 & $5.11 \mathrm{E}+002$ & $1.36 \mathrm{E}+003$ \\
\hline $\mathrm{M}$ & 3 & 1219.99 & 563.22 & $7.70 \mathrm{E}+001$ & $9.96 \mathrm{E}+001$ \\
\hline & 4 & 1233.07 & 569.27 & $1.15 \mathrm{E}+002$ & $1.58 \mathrm{E}+002$ \\
\hline & 5 & 1309.78 & 604.70 & $5.94 \mathrm{E}+002$ & $1.47 \mathrm{E}+002$ \\
\hline & 7 & 1433.00 & 661.62 & $3.72 \mathrm{E}+003$ & $1.19 \mathrm{E}+002$ \\
\hline & 8 & 1723.39 & 795.78 & $4.36 \mathrm{E}+002$ & $8.77 \mathrm{E}+001$ \\
\hline & 10 & 1807.73 & 834.75 & $3.23 \mathrm{E}+001$ & $8.07 \mathrm{E}+001$ \\
\hline
\end{tabular}

For unknown peaks we assume yield to be $100 \%$ and no decay correction is performed

$M=$ First peak in a multiplet region

$\mathrm{m}=$ Other peak in a multiplet region

$\mathrm{F}=$ Fitted singlet

\section{NUCLIDE RESULTS FOR Accuscan II <Summed>}

\begin{tabular}{|l|l|l|l|r|}
\hline & $\begin{array}{l}\text { Nuclide } \\
\text { Name }\end{array}$ & $\begin{array}{l}\text { Id } \\
\text { Confidence }\end{array}$ & $\begin{array}{l}\text { Wt Mean } \\
\text { Activity (nCi) }\end{array}$ & Error (1SD) \\
\hline MN-54 & 1.000 & $3.497251 \mathrm{E}+000$ & $57.13 \%$ \\
\hline CO-57 & 1.000 & $1.695289 \mathrm{E}+001$ & $18.27 \%$ \\
\hline CO-60 & 1.000 & $1.628637 \mathrm{E}+002$ & $2.34 \%$ \\
\hline CS-134 & 0.941 & $5.382743 \mathrm{E}+001$ & $4.03 \%$ \\
\hline CS-137 & 1.000 & $3.979652 \mathrm{E}+002$ & $2.07 \%$ \\
\hline
\end{tabular}

$?=$ Nuclide is part of an undetermined solution

$\mathrm{X}=$ Nuclide rejected by the interference analysis

$@$ @ = Nuclide contains energy lines not used in Weighted Mean Activity 
! = Nuclide was corrected for parent/daughter

NUCLIDE MDA RESULTS FOR Accuscan II <Summed>

\begin{tabular}{|c|l|r|r|l|}
\hline & $\begin{array}{l}\text { Nuclide } \\
\text { Name }\end{array}$ & Energy (keV) & Yield (\%) & \multicolumn{1}{l|}{$\begin{array}{l}\text { Line MDA } \\
\text { (nCi) }\end{array}$} \\
\hline$>$ & K-40 & 1460.81 & 10.67 & $0.0000 \mathrm{E}+000$ \\
\hline$>$ & CR-51 & 320.08 & 9.83 & $0.0000 \mathrm{E}+000$ \\
\hline$+>$ & MN-54 & $834.83 *$ & 99.97 & $0.0000 \mathrm{E}+000$ \\
\hline
\end{tabular}

User: David Georgeson 
Analysis Report - Lung Test [999-99-9996]

\section{NUCLIDE MDA RESULTS FOR Accuscan II <Summed>}

\begin{tabular}{|c|c|c|c|c|}
\hline & $\begin{array}{l}\text { Nuclide } \\
\text { Name }\end{array}$ & Energy (keV) & Yield (\%) & $\begin{array}{l}\text { Line MDA } \\
\text { (nCi) }\end{array}$ \\
\hline$+>$ & \multirow[t]{2}{*}{ CO-57 } & 122.06 * & 85.51 & $0.0000 \mathrm{E}+000$ \\
\hline$+>$ & & 136.48 & 10.60 & $0.0000 \mathrm{E}+000$ \\
\hline$>$ & CO-58 & 810.76 & 99.40 & $0.0000 \mathrm{E}+000$ \\
\hline$>$ & \multirow[t]{4}{*}{ FE-59 } & 142.65 & 1.03 & $0.0000 E+000$ \\
\hline$>$ & & 192.34 & 3.11 & $0.0000 \mathrm{E}+000$ \\
\hline$>$ & & 1099.22 & 56.50 & $0.0000 \mathrm{E}+000$ \\
\hline$>$ & & 1291.56 & 43.20 & $0.0000 \mathrm{E}+000$ \\
\hline$+>$ & \multirow[t]{2}{*}{ CO-60 } & 1173.22 * & 100.00 & $0.0000 E+000$ \\
\hline$+>$ & & 1332.49 * & 100.00 & $0.0000 E+000$ \\
\hline$>$ & ZN-65 & 1115.52 & 50.75 & $0.0000 \mathrm{E}+000$ \\
\hline$>$ & NB-95 & 765.79 & 99.81 & $0.0000 E+000$ \\
\hline$>$ & \multirow[t]{2}{*}{ ZR-95 } & 724.18 & 43.70 & $0.0000 E+000$ \\
\hline$>$ & & 756.72 & 55.30 & $0.0000 E+000$ \\
\hline$>$ & \multirow[t]{2}{*}{ RU-106 } & 621.84 & 9.80 & $0.0000 E+000$ \\
\hline$>$ & & 1050.47 & 1.73 & $0.0000 \mathrm{E}+000$ \\
\hline$>$ & \multirow[t]{15}{*}{ AG-110M } & 446.80 & 3.64 & $0.0000 \mathrm{E}+000$ \\
\hline$>$ & & 620.35 & 2.77 & $0.0000 \mathrm{E}+000$ \\
\hline$>$ & & 657.75 & 94.40 & $0.0000 \mathrm{E}+000$ \\
\hline$>$ & & 677.61 & 10.68 & $0.0000 \mathrm{E}+000$ \\
\hline$>$ & & 686.99 & 6.47 & $0.0000 \mathrm{E}+000$ \\
\hline$>$ & & 706.67 & 16.68 & $0.0000 \mathrm{E}+000$ \\
\hline$>$ & & 744.26 & 4.64 & $0.0000 \mathrm{E}+000$ \\
\hline$>$ & & 763.93 & 22.28 & $0.0000 \mathrm{E}+000$ \\
\hline$>$ & & 818.02 & 7.30 & $0.0000 E+000$ \\
\hline$>$ & & 884.67 & 72.60 & $0.0000 E+000$ \\
\hline$>$ & & 937.48 & 34.20 & $0.0000 E+000$ \\
\hline$>$ & & 1384.27 & 24.26 & $0.0000 \mathrm{E}+000$ \\
\hline$>$ & & 1475.76 & 3.97 & $0.0000 \mathrm{E}+000$ \\
\hline$>$ & & 1505.00 & 13.06 & $0.0000 \mathrm{E}+000$ \\
\hline$>$ & & 1562.27 & 1.18 & $0.0000 \mathrm{E}+000$ \\
\hline$>$ & \multirow[t]{5}{*}{$\mid-131$} & 80.18 & 2.62 & $0.0000 \mathrm{E}+000$ \\
\hline$>$ & & 284.30 & 6.05 & $0.0000 \mathrm{E}+000$ \\
\hline$>$ & & 364.48 & 81.20 & $0.0000 \mathrm{E}+000$ \\
\hline$>$ & & 636.97 & 7.26 & $0.0000 E+000$ \\
\hline$>$ & & 722.89 & 1.80 & $0.0000 E+000$ \\
\hline$>$ & \multirow[t]{2}{*}{$\mid-133$} & 529.87 & 86.30 & $0.0000 E+000$ \\
\hline$>$ & & 875.33 & 4.47 & $0.0000 E+000$ \\
\hline$+>$ & CS-134 & 475.35 & 1.46 & $0.0000 E+000$ \\
\hline
\end{tabular}




\begin{tabular}{|l|r|r|r|}
\hline$+>$ \\
\hline +>
\end{tabular}

User: David Georgeson 
Analysis Report - Lung Test [999-99-9996]

\section{NUCLIDE MDA RESULTS FOR Accuscan II <Summed>}

\begin{tabular}{|c|c|c|c|c|}
\hline & \begin{tabular}{|l|} 
Nuclide \\
Name
\end{tabular} & Energy (keV) & Yield (\%) & Line MDA (nCi) \\
\hline$+>$ & CS-137 & 661.65 * & 85.12 & $0.0000 \mathrm{E}+000$ \\
\hline$>$ & \multirow[t]{5}{*}{ BA-140 } & 162.64 & 6.70 & $0.0000 \mathrm{E}+000$ \\
\hline$>$ & & 304.84 & 4.50 & $0.0000 E+000$ \\
\hline$>$ & & 423.70 & 3.20 & $0.0000 \mathrm{E}+000$ \\
\hline$>$ & & 437.55 & 2.00 & $0.0000 \mathrm{E}+000$ \\
\hline$>$ & & 537.32 & 25.00 & $0.0000 \mathrm{E}+000$ \\
\hline$>$ & \multirow[t]{9}{*}{ LA-140 } & 328.77 & 20.50 & $0.0000 \mathrm{E}+000$ \\
\hline$>$ & & 432.53 & 2.94 & $0.0000 \mathrm{E}+000$ \\
\hline$>$ & & 487.03 & 45.50 & $0.0000 \mathrm{E}+000$ \\
\hline$>$ & & 751.79 & 4.40 & $0.0000 \mathrm{E}+000$ \\
\hline$>$ & & 815.85 & 23.50 & $0.0000 \mathrm{E}+000$ \\
\hline$>$ & & 867.82 & 5.63 & $0.0000 E+000$ \\
\hline$>$ & & 919.63 & 2.88 & $0.0000 \mathrm{E}+000$ \\
\hline$>$ & & 925.24 & 7.09 & $0.0000 \mathrm{E}+000$ \\
\hline$>$ & & 1596.49 & 95.49 & $0.0000 \mathrm{E}+000$ \\
\hline$>$ & CE-141 & 145.44 & 48.40 & $0.0000 \mathrm{E}+000$ \\
\hline$>$ & \multirow[t]{2}{*}{ CE-144 } & 80.11 & 1.60 & $0.0000 \mathrm{E}+000$ \\
\hline$>$ & & 133.54 & 10.80 & $0.0000 \mathrm{E}+000$ \\
\hline$>$ & \multirow[t]{11}{*}{ EU-152 } & 121.78 & 28.40 & $0.0000 \mathrm{E}+000$ \\
\hline$>$ & & 244.69 & 7.49 & $0.0000 \mathrm{E}+000$ \\
\hline$>$ & & 344.27 & 26.50 & $0.0000 \mathrm{E}+000$ \\
\hline$>$ & & 411.11 & 2.21 & $0.0000 \mathrm{E}+000$ \\
\hline$>$ & & 443.98 & 3.11 & $0.0000 \mathrm{E}+000$ \\
\hline$>$ & & 778.89 & 12.74 & $0.0000 \mathrm{E}+000$ \\
\hline$>$ & & 867.32 & 4.16 & $0.0000 \mathrm{E}+000$ \\
\hline$>$ & & 964.01 & 14.40 & $0.0000 E+000$ \\
\hline$>$ & & 1085.78 & 10.00 & $0.0000 \mathrm{E}+000$ \\
\hline$>$ & & 1112.02 & 13.30 & $0.0000 \mathrm{E}+000$ \\
\hline$>$ & & 1407.95 & 20.70 & $0.0000 \mathrm{E}+000$ \\
\hline
\end{tabular}

$+=$ Nuclide Identified during the nuclide identification

$*$ = Energy Line found in the spectrum

$>=$ MDA Value not calculated 


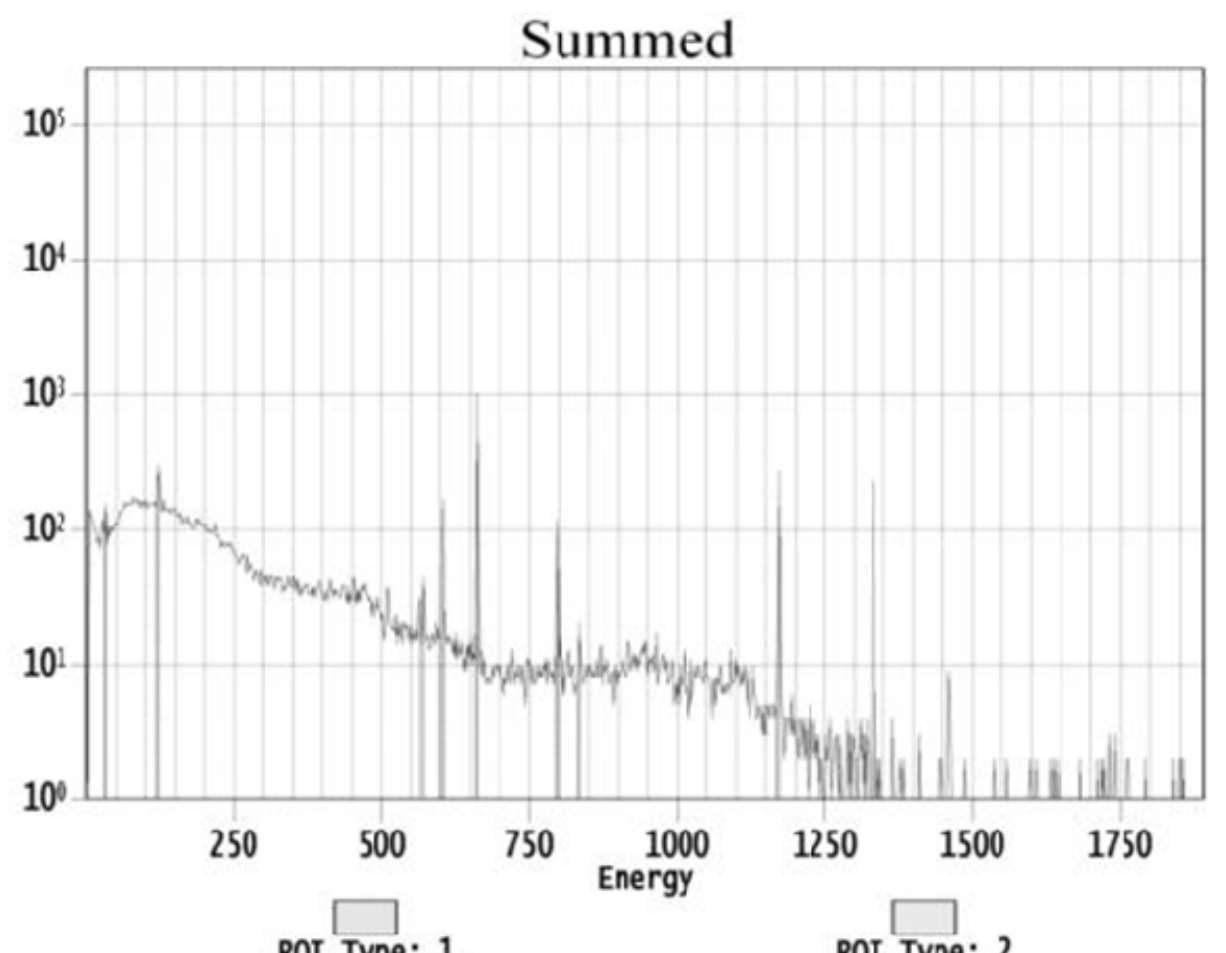

ROI Type: 1

ROI Type: 2 


\section{COUNT 4}

Apex-InVivo

\section{Analysis Report - Lung Test [999-99-9996]}

\section{SOURCE INFORMATION}

Primary ID

Last Name

First Name

Height (in)

Weight (Ib)

Chest Wall Thickness (cm)
999-99-9996

Test

Lung

\section{COUNTER INFORMATION}

\section{Counter Name \\ Counter Location \\ Configuration Name}

Accuscan II

Battelle

Lung - High Energy

\section{COUNT INFORMATION}

$\begin{array}{ll}\begin{array}{l}\text { Count Operation } \\ \text { Count Reason } \\ \text { Frequency } \\ \text { Comment }\end{array} & \begin{array}{l}\text { Individual } 10 \text { minutes } \\ \text { Routine operation }\end{array} \\ \text { Intake Date } & \text { MFP-1-001 Validation } \\ \text { Acquisition Started } & \\ \text { Acquisition Time } & 4 / 6 / 20119: 13: 21 \text { AM } \\ \text { Operator Name } & 602.28 \\ \text { Count Status } & \text { David Georgeson } \\ \text { Primary Review } & \text { Not Reviewed } \\ \text { Secondary Review } & \text { No } \\ \end{array}$

\section{DETECTOR COUNT RATE REPORT}

\begin{tabular}{|l|r|r|r|c|}
\hline Detector Name & $\begin{array}{c}\text { Count } \\
\text { Rate }\end{array}$ & $\begin{array}{c}\text { Count Rate } \\
\text { Range }\end{array}$ & Live Time & Analyzed \\
\hline Right & 83.80 & $1-4096$ & 600.0 & No \\
\hline Left & 80.31 & $1-4096$ & 600.0 & No \\
\hline Summed & 164.16 & $1-4096$ & 600.0 & Yes \\
\hline
\end{tabular}

Primary Review by:

Date:

User: David Georgeson 


\section{DETECTOR GROUP INFORMATION}

$\begin{array}{ll}\text { Detector Group Name } & \text { Summed } \\ \text { Detector Group Configuration } & \text { Lung Screening } \\ \text { Analysis Date } & 4 / 6 / 2011 \text { 9:13:11 AM } \\ \text { Energy Calibration } & \text { Energy Calibration (HE Lung) 4/5/2011 8:18 AM } \\ \text { Efficiency Calibration } & \text { Eff. Calibration (HE Lung Realistic)4/5/2011 4:05 PM (Dual) } \\ \quad \text { Multi Curve } & \text { No } \\ \text { Source Distribution } & \text { Lung } \\ \text { Analysis Sequence File } & \text { ROUNPPGE.ASF } \\ \text { Bkg Acq used for area correction } & \text { Not Performed } \\ \text { Nuclide Identification } & \\ \quad \text { Nuclide Identification Library } & \text { INLPLANTLIB.NLB } \\ \quad \text { Tentative NID Library } & \text { INLPLANTLIB.NLB } \\ \quad \text { Peak Search Library } & \text { INLPLANTLIB.NLB } \\ \quad \text { Analysis Limits (channels) } & \text { 40 - 4095 } \\ \quad \text { Energy Tolerance (FWHM) } & \text { 1.20 } \\ \text { Comment } & \end{array}$

PEAK SEARCH RESULTS FOR Accuscan II <Summed>

\begin{tabular}{|r|r|r|r|r|r||}
\hline \multicolumn{7}{|c||}{ Peak Analysis } \\
\hline & No & $\begin{array}{l}\text { Peak } \\
\text { Centroid }\end{array}$ & $\begin{array}{l}\text { Energy } \\
\text { (keV) }\end{array}$ & $\begin{array}{l}\text { Net Peak } \\
\text { Area }\end{array}$ & $\begin{array}{l}\text { Continuum } \\
\text { Counts }\end{array}$ \\
\hline & 1 & 70.15 & 32.29 & $3.04 \mathrm{E}+002$ & $6.40 \mathrm{E}+002$ \\
\hline & 2 & 264.64 & 122.07 & $4.59 \mathrm{E}+002$ & $1.32 \mathrm{E}+003$ \\
\hline & 3 & 1232.74 & 569.11 & $1.11 \mathrm{E}+002$ & $1.45 \mathrm{E}+002$ \\
\hline & 4 & 1309.64 & 604.63 & $5.69 \mathrm{E}+002$ & $1.68 \mathrm{E}+002$ \\
\hline & 5 & 1432.96 & 661.60 & $3.58 \mathrm{E}+003$ & $1.81 \mathrm{E}+002$ \\
\hline & 6 & 1723.50 & 795.83 & $3.83 \mathrm{E}+002$ & $1.09 \mathrm{E}+002$ \\
\hline & 7 & 2539.95 & 1173.16 & $1.16 \mathrm{E}+003$ & $5.88 \mathrm{E}+001$ \\
\hline & 8 & 2884.31 & 1332.36 & $1.06 \mathrm{E}+003$ & $2.12 \mathrm{E}+001$ \\
\hline
\end{tabular}

For unknown peaks we assume yield to be $100 \%$ and no decay correction is performed.

$M=$ First peak in a multiplet region

$\mathrm{m}=$ Other peak in a multiplet region

$\mathrm{F}=$ Fitted singlet

NUCLIDE RESULTS FOR Accuscan II <Summed>

\begin{tabular}{|l|l|r|r|r|}
\hline & $\begin{array}{l}\text { Nuclide } \\
\text { Name }\end{array}$ & $\begin{array}{l}\text { Id } \\
\text { Confidence }\end{array}$ & $\begin{array}{l}\text { Wt Mean } \\
\text { Activity (nCi) }\end{array}$ & Error (1SD) \\
\hline K-40 & 0.999 & $8.732788 \mathrm{E}+00$ & $18.93 \%$ \\
\hline CO-57 & 1.000 & $1.522031 \mathrm{E}+00$ & $19.29 \%$ \\
\hline CO-60 & 1.000 & $1.601501 \mathrm{E}+00$ & $2.43 \%$ \\
\hline CS-134 & 0.935 & $4.864050 \mathrm{E}+00$ & $4.88 \%$ \\
\hline CS-137 & 1.000 & $3.825975 \mathrm{E}+00$ & $2.15 \%$ \\
\hline
\end{tabular}

$?=$ Nuclide is part of an undetermined solution

$\mathrm{X}=$ Nuclide rejected by the interference analysis

$@$ = Nuclide contains energy lines not used in Weighted Mean Activity

! = Nuclide was corrected for parent/daughter 
NUCLIDE MDA RESULTS FOR Accuscan II <Summed>

\begin{tabular}{|c|l|r|r|r|}
\hline & Nuclide & Energy (keV) & \multicolumn{1}{|l|}{ Yield (\%) } & \multicolumn{1}{l|}{ Line MDA } \\
\hline$+>$ & K-40 & 1460.81 & 10.67 & $0.0000 \mathrm{E}+000$ \\
\hline$>$ & CR-51 & 320.08 & 9.83 & $0.0000 \mathrm{E}+000$ \\
\hline$>$ & MN-54 & 834.83 & 99.97 & $0.0000 \mathrm{E}+000$ \\
\hline$+>$ & CO-57 & $122.06 *$ & 85.51 & $0.0000 \mathrm{E}+000$ \\
\hline
\end{tabular}

User: David Georgeson 
Analysis Report - Lung Test [999-99-9996]

\section{NUCLIDE MDA RESULTS FOR Accuscan II <Summed>}

\begin{tabular}{|c|c|c|c|c|}
\hline & \begin{tabular}{|l|} 
Nuclide \\
Name
\end{tabular} & Energy (keV) & Yield (\%) & $\begin{array}{l}\text { Line MDA } \\
\text { (nCi) }\end{array}$ \\
\hline$+>$ & CO-57 & 136.48 & 10.60 & $0.0000 E+000$ \\
\hline$>$ & CO-58 & 810.76 & 99.40 & $0.0000 E+000$ \\
\hline$>$ & \multirow[t]{4}{*}{ FE-59 } & 142.65 & 1.03 & $0.0000 E+000$ \\
\hline$>$ & & 192.34 & 3.11 & $0.0000 E+000$ \\
\hline$>$ & & 1099.22 & 56.50 & $0.0000 E+000$ \\
\hline$>$ & & 1291.56 & 43.20 & $0.0000 \mathrm{E}+000$ \\
\hline$+>$ & \multirow[t]{2}{*}{ CO-60 } & 1173.22 * & 100.00 & $0.0000 \mathrm{E}+000$ \\
\hline$+>$ & & $1332.49 *$ & 100.00 & $0.0000 E+000$ \\
\hline$>$ & ZN-65 & 1115.52 & 50.75 & $0.0000 E+000$ \\
\hline$>$ & NB-95 & 765.79 & 99.81 & $0.0000 \mathrm{E}+000$ \\
\hline$>$ & \multirow[t]{2}{*}{ ZR-95 } & 724.18 & 43.70 & $0.0000 E+000$ \\
\hline$>$ & & 756.72 & 55.30 & $0.0000 E+000$ \\
\hline$>$ & \multirow[t]{2}{*}{ RU-106 } & 621.84 & 9.80 & $0.0000 \mathrm{E}+000$ \\
\hline$>$ & & 1050.47 & 1.73 & $0.0000 \mathrm{E}+000$ \\
\hline$>$ & \multirow[t]{15}{*}{ AG-110M } & 446.80 & 3.64 & $0.0000 \mathrm{E}+000$ \\
\hline$>$ & & 620.35 & 2.77 & $0.0000 \mathrm{E}+000$ \\
\hline$>$ & & 657.75 & 94.40 & $0.0000 E+000$ \\
\hline$>$ & & 677.61 & 10.68 & $0.0000 \mathrm{E}+000$ \\
\hline$>$ & & 686.99 & 6.47 & $0.0000 \mathrm{E}+000$ \\
\hline$>$ & & 706.67 & 16.68 & $0.0000 E+000$ \\
\hline$>$ & & 744.26 & 4.64 & $0.0000 \mathrm{E}+000$ \\
\hline$>$ & & 763.93 & 22.28 & $0.0000 \mathrm{E}+000$ \\
\hline$>$ & & 818.02 & 7.30 & $0.0000 \mathrm{E}+000$ \\
\hline$>$ & & 884.67 & 72.60 & $0.0000 E+000$ \\
\hline$>$ & & 937.48 & 34.20 & $0.0000 \mathrm{E}+000$ \\
\hline$>$ & & 1384.27 & 24.26 & $0.0000 E+000$ \\
\hline$>$ & & 1475.76 & 3.97 & $0.0000 \mathrm{E}+000$ \\
\hline$>$ & & 1505.00 & 13.06 & $0.0000 E+000$ \\
\hline$>$ & & 1562.27 & 1.18 & $0.0000 \mathrm{E}+000$ \\
\hline$>$ & \multirow[t]{5}{*}{ I-131 } & 80.18 & 2.62 & $0.0000 \mathrm{E}+000$ \\
\hline$>$ & & 284.30 & 6.05 & $0.0000 E+000$ \\
\hline$>$ & & 364.48 & 81.20 & $0.0000 \mathrm{E}+000$ \\
\hline$>$ & & 636.97 & 7.26 & $0.0000 \mathrm{E}+000$ \\
\hline$>$ & & 722.89 & 1.80 & $0.0000 E+000$ \\
\hline$>$ & \multirow[t]{2}{*}{ I-133 } & 529.87 & 86.30 & $0.0000 \mathrm{E}+000$ \\
\hline$>$ & & 875.33 & 4.47 & $0.0000 \mathrm{E}+000$ \\
\hline$+>$ & \multirow[t]{2}{*}{ CS-134 } & 475.35 & 1.46 & $0.0000 \mathrm{E}+000$ \\
\hline$+>$ & & 563.23 & 8.38 & $0.0000 \mathrm{E}+000$ \\
\hline
\end{tabular}




\begin{tabular}{|c|c|c|c|c|}
\hline$+>$ & & 569.32 * & 15.43 & $0.0000 \mathrm{E}+000$ \\
\hline$+>$ & & 604.70 * & 97.60 & $0.0000 \mathrm{E}+000$ \\
\hline$+>$ & & 795.84 * & 85.40 & $0.0000 \mathrm{E}+000$ \\
\hline$+>$ & & 801.93 & 8.73 & $0.0000 \mathrm{E}+000$ \\
\hline$+>$ & & 1038.57 & 1.00 & $0.0000 \mathrm{E}+000$ \\
\hline$+>$ & & 1167.94 & 1.80 & $0.0000 \mathrm{E}+000$ \\
\hline$+>$ & & 1365.15 & 3.04 & $0.0000 \mathrm{E}+000$ \\
\hline$+>$ & CS-137 & 661.65 * & 85.12 & $0.0000 \mathrm{E}+000$ \\
\hline
\end{tabular}

User: David Georgeson 
Analysis Report - Lung Test [999-99-9996]

\section{NUCLIDE MDA RESULTS FOR Accuscan II <Summed>}

\begin{tabular}{|c|c|c|c|c|}
\hline & Nuclide & Energy (keV) & Yield (\%) & Line MDA \\
\hline$>$ & \multirow[t]{5}{*}{ BA-140 } & 162.64 & 6.70 & $0.0000 \mathrm{E}+000$ \\
\hline$>$ & & 304.84 & 4.50 & $0.0000 E+000$ \\
\hline$>$ & & 423.70 & 3.20 & $0.0000 E+000$ \\
\hline$>$ & & 437.55 & 2.00 & $0.0000 \mathrm{E}+000$ \\
\hline$>$ & & 537.32 & 25.00 & $0.0000 \mathrm{E}+000$ \\
\hline$>$ & \multirow[t]{9}{*}{ LA-140 } & 328.77 & 20.50 & $0.0000 E+000$ \\
\hline$>$ & & 432.53 & 2.94 & $0.0000 \mathrm{E}+000$ \\
\hline$>$ & & 487.03 & 45.50 & $0.0000 \mathrm{E}+000$ \\
\hline$>$ & & 751.79 & 4.40 & $0.0000 \mathrm{E}+000$ \\
\hline$>$ & & 815.85 & 23.50 & $0.0000 \mathrm{E}+000$ \\
\hline$>$ & & 867.82 & 5.63 & $0.0000 \mathrm{E}+000$ \\
\hline$>$ & & 919.63 & 2.88 & $0.0000 \mathrm{E}+000$ \\
\hline$>$ & & 925.24 & 7.09 & $0.0000 E+000$ \\
\hline$>$ & & 1596.49 & 95.49 & $0.0000 \mathrm{E}+000$ \\
\hline$>$ & CE-141 & 145.44 & 48.40 & $0.0000 \mathrm{E}+000$ \\
\hline$>$ & \multirow[t]{2}{*}{ CE-144 } & 80.11 & 1.60 & $0.0000 \mathrm{E}+000$ \\
\hline$>$ & & 133.54 & 10.80 & $0.0000 \mathrm{E}+000$ \\
\hline$>$ & \multirow[t]{11}{*}{ EU-152 } & 121.78 & 28.40 & $0.0000 \mathrm{E}+000$ \\
\hline$>$ & & 244.69 & 7.49 & $0.0000 \mathrm{E}+000$ \\
\hline$>$ & & 344.27 & 26.50 & $0.0000 \mathrm{E}+000$ \\
\hline$>$ & & 411.11 & 2.21 & $0.0000 \mathrm{E}+000$ \\
\hline$>$ & & 443.98 & 3.11 & $0.0000 \mathrm{E}+000$ \\
\hline$>$ & & 778.89 & 12.74 & $0.0000 \mathrm{E}+000$ \\
\hline$>$ & & 867.32 & 4.16 & $0.0000 \mathrm{E}+000$ \\
\hline$>$ & & 964.01 & 14.40 & $0.0000 \mathrm{E}+000$ \\
\hline$>$ & & 1085.78 & 10.00 & $0.0000 \mathrm{E}+000$ \\
\hline$>$ & & 1112.02 & 13.30 & $0.0000 \mathrm{E}+000$ \\
\hline$>$ & & 1407.95 & 20.70 & $0.0000 E+000$ \\
\hline
\end{tabular}

User: David Georgeson 
Analysis Report - Lung Test [999-99-9996]

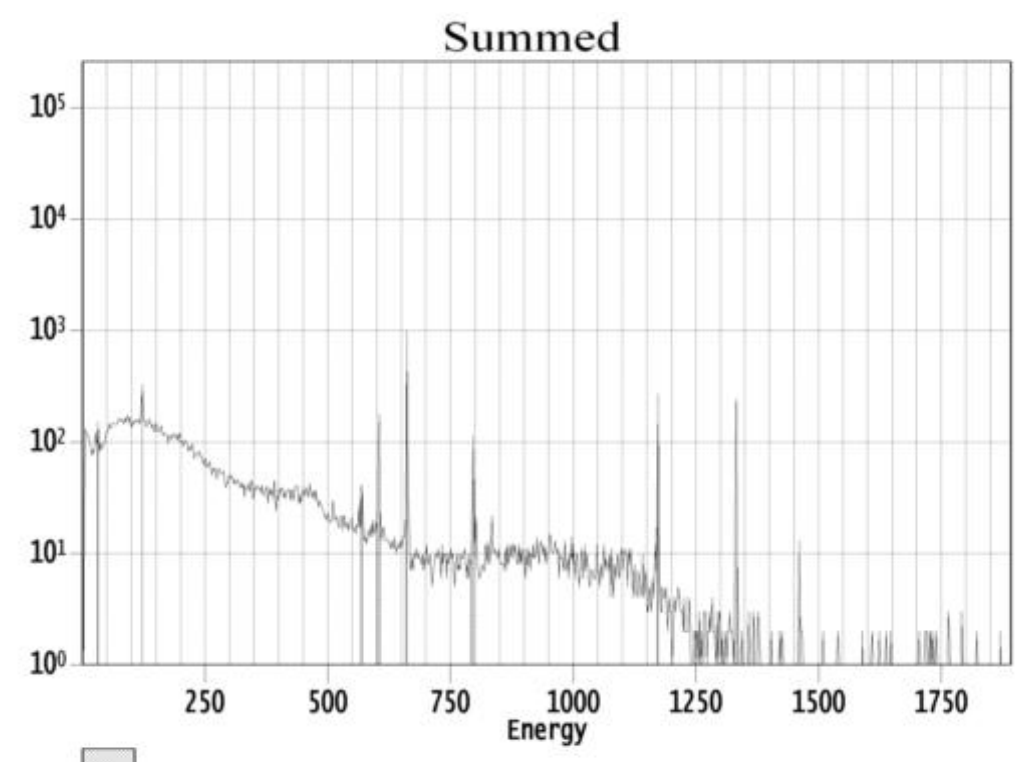

ROI Type: 1

User: David Georgeson 


\section{COUNT 5}

\section{Apex-InVivo}

\section{Analysis Report - Lung Test [999-99-9996]}

\section{SOURCE INFORMATION}

Primary ID

Last Name

999-99-9996

First Name

Test

Height (in)

Lung

Weight (lb)

Chest Wall Thickness (cm)

\section{COUNTER INFORMATION}

\section{Counter Name \\ Counter Location \\ Configuration Name}

\section{COUNT INFORMATION}

Count Operation

Count Reason

Frequency

Comment

Intake Date

Acquisition Started

Acquisition Time

Operator Name

Count Status

Primary Review

Secondary Review
Accuscan II

Battelle

Lung - High Energy

\section{DETECTOR COUNT RATE REPORT}

\begin{tabular}{|l|r|c|c|c|}
\hline \multicolumn{1}{|c|}{ Detector Name } & $\begin{array}{c}\text { Count } \\
\text { Rate }\end{array}$ & $\begin{array}{c}\text { Count } \\
\text { Rate }\end{array}$ & Live Time & Analyzed \\
\hline Left & 81.57 & $1-4096$ & 600.0 & No \\
\hline Right & 82.33 & $1-4096$ & 600.0 & No \\
\hline Summed & 163.63 & $1-4096$ & 600.0 & Yes \\
\hline
\end{tabular}

Primary Review by:

Date:

User: David Georgeson

Individual 10 minutes

Routine operation

MFP-1-001 validation count \#5

4/6/2011 9:35:00 AM

602.3099999

David Georgeson

Not Reviewed

No

No 


\section{DETECTOR GROUP INFORMATION}

$\begin{array}{ll}\text { Detector Group Name } & \text { Summed } \\ \text { Detector Group Configuration } & \text { Lung Screening } \\ \text { Analysis Date } & 4 / 6 / 2011 \text { 9:34:50 AM } \\ \text { Energy Calibration } & \text { Energy Calibration (HE Lung) 4/5/2011 8:18 AM } \\ \text { Efficiency Calibration } & \text { Eff. Calibration (HE Lung Realistic)4/5/2011 4:05 PM (Dual) } \\ \quad \text { Multi Curve } & \text { No } \\ \text { Source Distribution } & \text { Lung } \\ \text { Analysis Sequence File } & \text { ROUNPPGE.ASF } \\ \text { Bkg Acq used for area correction } & \text { Not Performed } \\ \text { Nuclide Identification } & \\ \quad \text { Nuclide Identification Library } & \text { INLPLANTLIB.NLB } \\ \quad \text { Tentative NID Library } & \text { INLPLANTLIB.NLB } \\ \quad \text { Peak Search Library } & \text { INLPLANTLIB.NLB } \\ \quad \text { Analysis Limits (channels) } & \text { 40 - 4095 } \\ \quad \text { Energy Tolerance (FWHM) } & 1.20 \\ \text { Comment } & \end{array}$

PEAK SEARCH RESULTS FOR Accuscan II <Summed>

\begin{tabular}{|r|r|r|r|r|r|}
\hline \multicolumn{7}{|c|}{ Peak Analysis } \\
\hline & No & $\begin{array}{l}\text { Peak } \\
\text { Centroid }\end{array}$ & $\begin{array}{l}\text { Energy } \\
\text { (keV) }\end{array}$ & $\begin{array}{l}\text { Net Peak } \\
\text { Area }\end{array}$ & $\begin{array}{l}\text { Continuum } \\
\text { Counts }\end{array}$ \\
\hline & 1 & 264.76 & 122.13 & $5.69 \mathrm{E}+002$ & $1.18 \mathrm{E}+003$ \\
\hline & 2 & 1233.05 & 569.25 & $8.47 \mathrm{E}+001$ & $1.45 \mathrm{E}+002$ \\
\hline & 3 & 1309.72 & 604.67 & $5.93 \mathrm{E}+002$ & $1.74 \mathrm{E}+002$ \\
\hline & 4 & 1433.03 & 661.64 & $3.66 \mathrm{E}+003$ & $1.09 \mathrm{E}+002$ \\
\hline & 5 & 1723.48 & 795.82 & $4.06 \mathrm{E}+002$ & $8.82 \mathrm{E}+001$ \\
\hline & 6 & 2539.80 & 1173.09 & $1.18 \mathrm{E}+003$ & $3.64 \mathrm{E}+001$ \\
\hline & 7 & 2884.44 & 1332.42 & $1.02 \mathrm{E}+003$ & $1.27 \mathrm{E}+001$ \\
\hline & 8 & 3161.76 & 1460.65 & $5.30 \mathrm{E}+001$ & $7.00 \mathrm{E}+000$ \\
\hline
\end{tabular}

For unknown peaks we assume yield to be $100 \%$ and no decay correction is performed

$M=$ First peak in a multiplet region

$\mathrm{m}=$ Other peak in a multiplet region

$\mathrm{F}=$ Fitted singlet

NUCLIDE RESULTS FOR Accuscan II <Summed>

\begin{tabular}{|l|l|r|r|r|}
\hline $\begin{array}{l}\text { Nuclide } \\
\text { Name }\end{array}$ & $\begin{array}{l}\text { Id } \\
\text { Confidence }\end{array}$ & $\begin{array}{l}\text { Wt Mean } \\
\text { Activity (nCi) }\end{array}$ & Error (1SD) \\
\hline K-40 & 0.999 & $8.196945 \mathrm{E}+00$ & $17.52 \%$ \\
\hline CO-57 & 1.000 & $1.888056 \mathrm{E}+00$ & $14.85 \%$ \\
\hline CO-60 & 1.000 & $1.592995 \mathrm{E}+00$ & $2.37 \%$ \\
\hline CS-134 & 1.000 & $5.044263 \mathrm{E}+00$ & $4.65 \%$ \\
\hline CS-137 & 1.000 & $3.915122 \mathrm{E}+00$ & $2.07 \%$ \\
\hline
\end{tabular}


NUCLIDE MDA RESULTS FOR Accuscan II <Summed>

\begin{tabular}{|c|l|r|r|l|}
\hline & $\begin{array}{l}\text { Nuclide } \\
\text { Name }\end{array}$ & Energy (keV) & Yield (\%) & $\begin{array}{l}\text { Line MDA } \\
\text { (nCi) }\end{array}$ \\
\hline$+>$ & K-40 & $1460.81 *$ & 10.67 & $0.0000 \mathrm{E}+000$ \\
\hline$>$ & CR-51 & 320.08 & 9.83 & $0.0000 \mathrm{E}+000$ \\
\hline$>$ & MN-54 & 834.83 & 99.97 & $0.0000 \mathrm{E}+000$ \\
\hline y & CO-57 & $122.06 *$ & 85.51 & $0.0000 \mathrm{E}+000$ \\
\cline { 1 - 3 }$+>$ & & 136.48 & 10.60 & $0.0000 \mathrm{E}+000$ \\
\hline
\end{tabular}

User: David Georgeson 
Analysis Report - Lung Test [999-99-9996]

NUCLIDE MDA RESULTS FOR Accuscan II <Summed>

\begin{tabular}{|c|c|c|c|c|}
\hline & \begin{tabular}{|l|} 
Nuclide \\
Name
\end{tabular} & Energy (keV) & Yield (\%) & Line MDA (nCi) \\
\hline$>$ & CO-58 & 810.76 & 99.40 & $0.0000 \mathrm{E}+000$ \\
\hline$>$ & \multirow[t]{4}{*}{ FE-59 } & 142.65 & 1.03 & $0.0000 \mathrm{E}+000$ \\
\hline$>$ & & 192.34 & 3.11 & $0.0000 \mathrm{E}+000$ \\
\hline$>$ & & 1099.22 & 56.50 & $0.0000 E+000$ \\
\hline$>$ & & 1291.56 & 43.20 & $0.0000 E+000$ \\
\hline$+>$ & \multirow[t]{2}{*}{ CO-60 } & $1173.22 *$ & 100.00 & $0.0000 \mathrm{E}+000$ \\
\hline$+>$ & & $1332.49 *$ & 100.00 & $0.0000 \mathrm{E}+000$ \\
\hline$>$ & ZN-65 & 1115.52 & 50.75 & $0.0000 \mathrm{E}+000$ \\
\hline$>$ & NB-95 & 765.79 & 99.81 & $0.0000 \mathrm{E}+000$ \\
\hline$>$ & \multirow[t]{2}{*}{ ZR-95 } & 724.18 & 43.70 & $0.0000 \mathrm{E}+000$ \\
\hline$>$ & & 756.72 & 55.30 & $0.0000 E+000$ \\
\hline$>$ & \multirow[t]{2}{*}{ RU-106 } & 621.84 & 9.80 & $0.0000 \mathrm{E}+000$ \\
\hline$>$ & & 1050.47 & 1.73 & $0.0000 \mathrm{E}+000$ \\
\hline$>$ & \multirow[t]{15}{*}{ AG-110M } & 446.80 & 3.64 & $0.0000 \mathrm{E}+000$ \\
\hline$>$ & & 620.35 & 2.77 & $0.0000 \mathrm{E}+000$ \\
\hline$>$ & & 657.75 & 94.40 & $0.0000 \mathrm{E}+000$ \\
\hline$>$ & & 677.61 & 10.68 & $0.0000 \mathrm{E}+000$ \\
\hline$>$ & & 686.99 & 6.47 & $0.0000 \mathrm{E}+000$ \\
\hline$>$ & & 706.67 & 16.68 & $0.0000 \mathrm{E}+000$ \\
\hline$>$ & & 744.26 & 4.64 & $0.0000 \mathrm{E}+000$ \\
\hline$>$ & & 763.93 & 22.28 & $0.0000 \mathrm{E}+000$ \\
\hline$>$ & & 818.02 & 7.30 & $0.0000 \mathrm{E}+000$ \\
\hline$>$ & & 884.67 & 72.60 & $0.0000 \mathrm{E}+000$ \\
\hline$>$ & & 937.48 & 34.20 & $0.0000 E+000$ \\
\hline$>$ & & 1384.27 & 24.26 & $0.0000 \mathrm{E}+000$ \\
\hline$>$ & & 1475.76 & 3.97 & $0.0000 \mathrm{E}+000$ \\
\hline$>$ & & 1505.00 & 13.06 & $0.0000 \mathrm{E}+000$ \\
\hline$>$ & & 1562.27 & 1.18 & $0.0000 \mathrm{E}+000$ \\
\hline$>$ & \multirow[t]{5}{*}{$\mid-131$} & 80.18 & 2.62 & $0.0000 \mathrm{E}+000$ \\
\hline$>$ & & 284.30 & 6.05 & $0.0000 \mathrm{E}+000$ \\
\hline$>$ & & 364.48 & 81.20 & $0.0000 \mathrm{E}+000$ \\
\hline$>$ & & 636.97 & 7.26 & $0.0000 \mathrm{E}+000$ \\
\hline$>$ & & 722.89 & 1.80 & $0.0000 \mathrm{E}+000$ \\
\hline$>$ & \multirow[t]{2}{*}{$\mid-133$} & 529.87 & 86.30 & $0.0000 \mathrm{E}+000$ \\
\hline$>$ & & 875.33 & 4.47 & $0.0000 \mathrm{E}+000$ \\
\hline$+>$ & \multirow[t]{3}{*}{ CS-134 } & 475.35 & 1.46 & $0.0000 \mathrm{E}+000$ \\
\hline$+>$ & & 563.23 & 8.38 & $0.0000 \mathrm{E}+000$ \\
\hline$+>$ & & $569.32 *$ & 15.43 & $0.0000 E+000$ \\
\hline
\end{tabular}




\begin{tabular}{|c|c|c|c|c|}
\hline$+>$ & & 604.70 * & 97.60 & $0.0000 E+000$ \\
\hline$+>$ & & 795.84 * & 85.40 & $0.0000 \mathrm{E}+000$ \\
\hline$+>$ & & 801.93 & 8.73 & $0.0000 E+000$ \\
\hline$+>$ & & 1038.57 & 1.00 & $0.0000 \mathrm{E}+000$ \\
\hline$+>$ & & 1167.94 & 1.80 & $0.0000 \mathrm{E}+000$ \\
\hline$+>$ & & 1365.15 & 3.04 & $0.0000 \mathrm{E}+000$ \\
\hline$+>$ & CS-137 & 661.65 * & 85.12 & $0.0000 \mathrm{E}+000$ \\
\hline$>$ & BA-140 & 162.64 & 6.70 & $0.0000 \mathrm{E}+000$ \\
\hline
\end{tabular}

User: David Georgeson 
Analysis Report - Lung Test [999-99-9996]

\section{NUCLIDE MDA RESULTS FOR Accuscan II <Summed>}

\begin{tabular}{|c|c|c|c|c|}
\hline & \begin{tabular}{|l|} 
Nuclide \\
Name
\end{tabular} & Energy (keV) & Yield (\%) & Line MDA (nCi) \\
\hline$>$ & \multirow[t]{4}{*}{ BA-140 } & 304.84 & 4.50 & $0.0000 \mathrm{E}+000$ \\
\hline$>$ & & 423.70 & 3.20 & $0.0000 \mathrm{E}+000$ \\
\hline$>$ & & 437.55 & 2.00 & $0.0000 \mathrm{E}+000$ \\
\hline$>$ & & 537.32 & 25.00 & $0.0000 \mathrm{E}+000$ \\
\hline$>$ & \multirow[t]{9}{*}{ LA-140 } & 328.77 & 20.50 & $0.0000 \mathrm{E}+000$ \\
\hline$>$ & & 432.53 & 2.94 & $0.0000 \mathrm{E}+000$ \\
\hline$>$ & & 487.03 & 45.50 & $0.0000 \mathrm{E}+000$ \\
\hline$>$ & & 751.79 & 4.40 & $0.0000 E+000$ \\
\hline$>$ & & 815.85 & 23.50 & $0.0000 \mathrm{E}+000$ \\
\hline$>$ & & 867.82 & 5.63 & $0.0000 \mathrm{E}+000$ \\
\hline$>$ & & 919.63 & 2.88 & $0.0000 \mathrm{E}+000$ \\
\hline$>$ & & 925.24 & 7.09 & $0.0000 \mathrm{E}+000$ \\
\hline$>$ & & 1596.49 & 95.49 & $0.0000 \mathrm{E}+000$ \\
\hline$>$ & CE-141 & 145.44 & 48.40 & $0.0000 \mathrm{E}+000$ \\
\hline$>$ & \multirow[t]{2}{*}{ CE-144 } & 80.11 & 1.60 & $0.0000 \mathrm{E}+000$ \\
\hline$>$ & & 133.54 & 10.80 & $0.0000 \mathrm{E}+000$ \\
\hline$>$ & \multirow[t]{11}{*}{ EU-152 } & 121.78 & 28.40 & $0.0000 \mathrm{E}+000$ \\
\hline$>$ & & 244.69 & 7.49 & $0.0000 \mathrm{E}+000$ \\
\hline$>$ & & 344.27 & 26.50 & $0.0000 \mathrm{E}+000$ \\
\hline$>$ & & 411.11 & 2.21 & $0.0000 \mathrm{E}+000$ \\
\hline$>$ & & 443.98 & 3.11 & $0.0000 \mathrm{E}+000$ \\
\hline$>$ & & 778.89 & 12.74 & $0.0000 \mathrm{E}+000$ \\
\hline$>$ & & 867.32 & 4.16 & $0.0000 \mathrm{E}+000$ \\
\hline$>$ & & 964.01 & 14.40 & $0.0000 \mathrm{E}+000$ \\
\hline$>$ & & 1085.78 & 10.00 & $0.0000 \mathrm{E}+000$ \\
\hline$>$ & & 1112.02 & 13.30 & $0.0000 E+000$ \\
\hline$>$ & & 1407.95 & 20.70 & $0.0000 \mathrm{E}+000$ \\
\hline
\end{tabular}

$+=$ Nuclide Identified during the nuclide identification

$*$ = Energy Line found in the spectrum

$>=$ MDA Value not calculated 


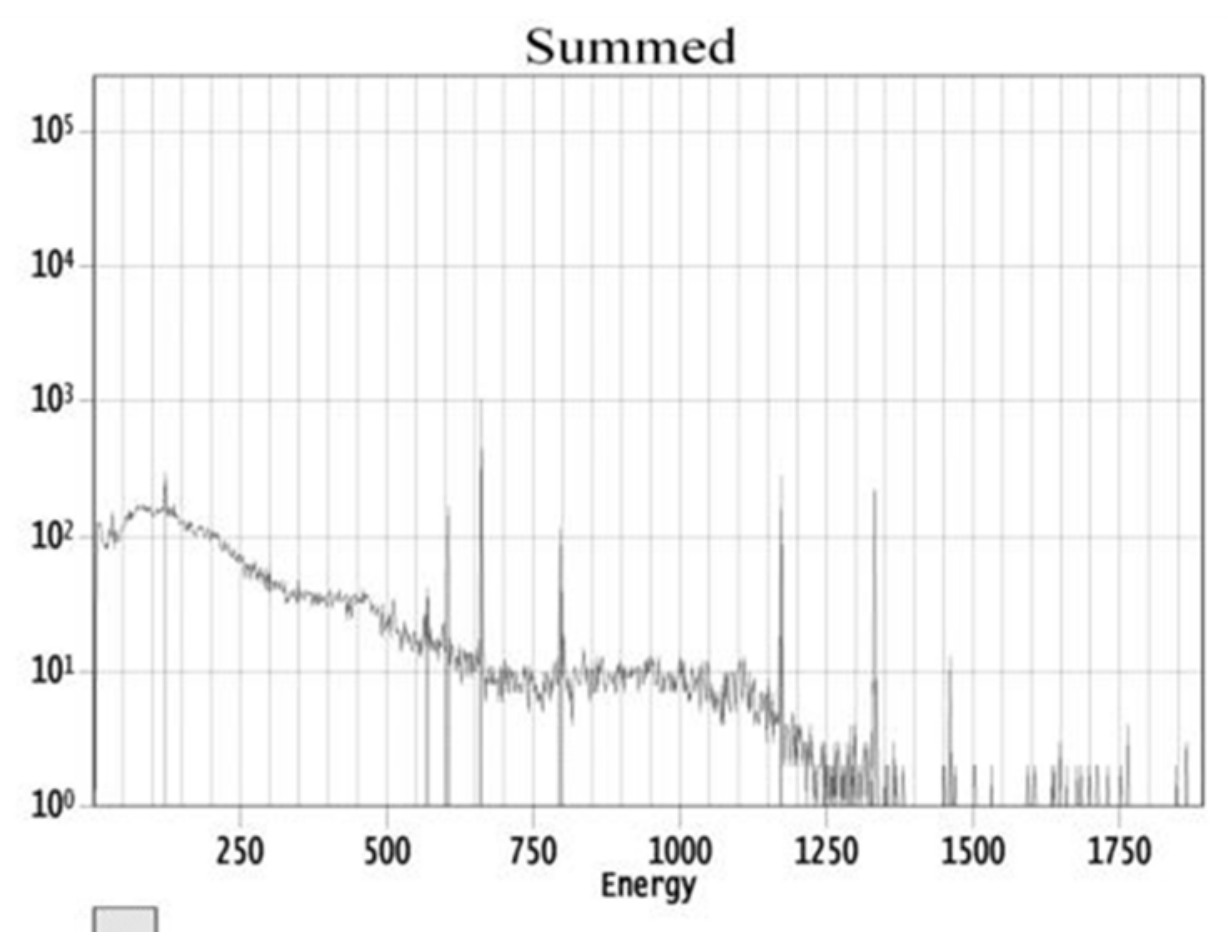

ROI Type: 1 


\section{Appendix I}

\section{CWI Realistic Torso Phantom}




\title{
Appendix I \\ CWI Realistic Torso Phantom
}

IAEA-SM-229/56

\author{
A TISSUE-EQUIVALENT TORSO PHANTOM* \\ For intercalibration of in-vivo, \\ transuranic-nuclide counting facilities
}

R.V, GRIFFITH, P,N. DEA.N, A.L. ANDERSON, J.C. FISHLK

Lawrence Livermore Laboratery, University of California.

Livermore, California.

Unjted States of America

Abscract

A TISSUE EOUVALENT TORSO PHANTOM FOR INTERCALIBRATJON OF IN-VLVO, TRANSURANIC.NLCLIDE COUNTING FACILITIES.

A tissuc-ęuivalent human-torso phantom has keea sonstcuesed for calibation of Il: counting systems used for in-vivo mesureauent of transtranie nuclides. The phantsr. contairs a humen male rib cage, remowable model oreans, end uciudes zissue-zquivslent chest fiares that sa be placed over the torso to simulete pesple with a wide zange of statures. The corgans includec are Junes, heart, liver, kidneys, spieen, and tracheo-bzonchial jymph nodes. Polyurethans with different concentrations of eslesam. carhonate was used to sunulate the lisear photoe-ztteruation properties of various human lissues - iean mussle, adipose-lissucimbscle mixtures and carvilage. Foaned polyozethane with catciuan cbrbonate simulates lung tissue. Transuasic jsotopes cas he insorporated unifor $x_{\text {y }}$ in the phantocis lunes ane other polyurethane-based oneans by dissolution of the atrate form, in acetane wilh larthankm n:tra:e

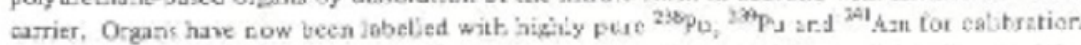
measarements. This phantora is the fust of thres that wil ae as:d in a Linited Stzles Deyait-

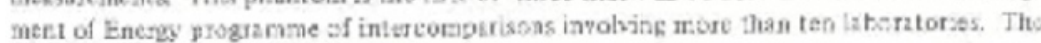

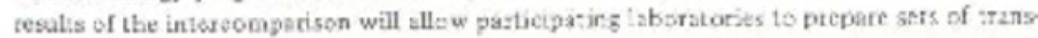
mission cunes that can be uged to predict tae pedfercince of their countire systems for a

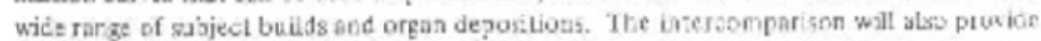

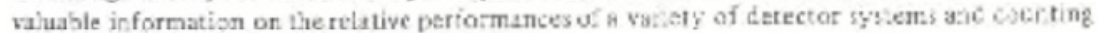
toshrigues.

\section{INTRODLCIION}

One of the most diflicult heatth-physics protilems is the accurete an:i ser vitive in vove messurement of ${ }^{239}$, act other transurark. auclides in the

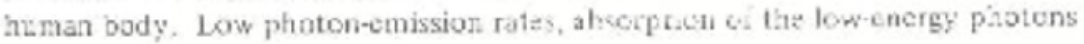

- Work periarmed under the auspices of the Uaited Statea Depart nent of Eaurgy by the Lawzence Linermore Laboratary undez contiact rumoer W-7405-Eeg-48. 


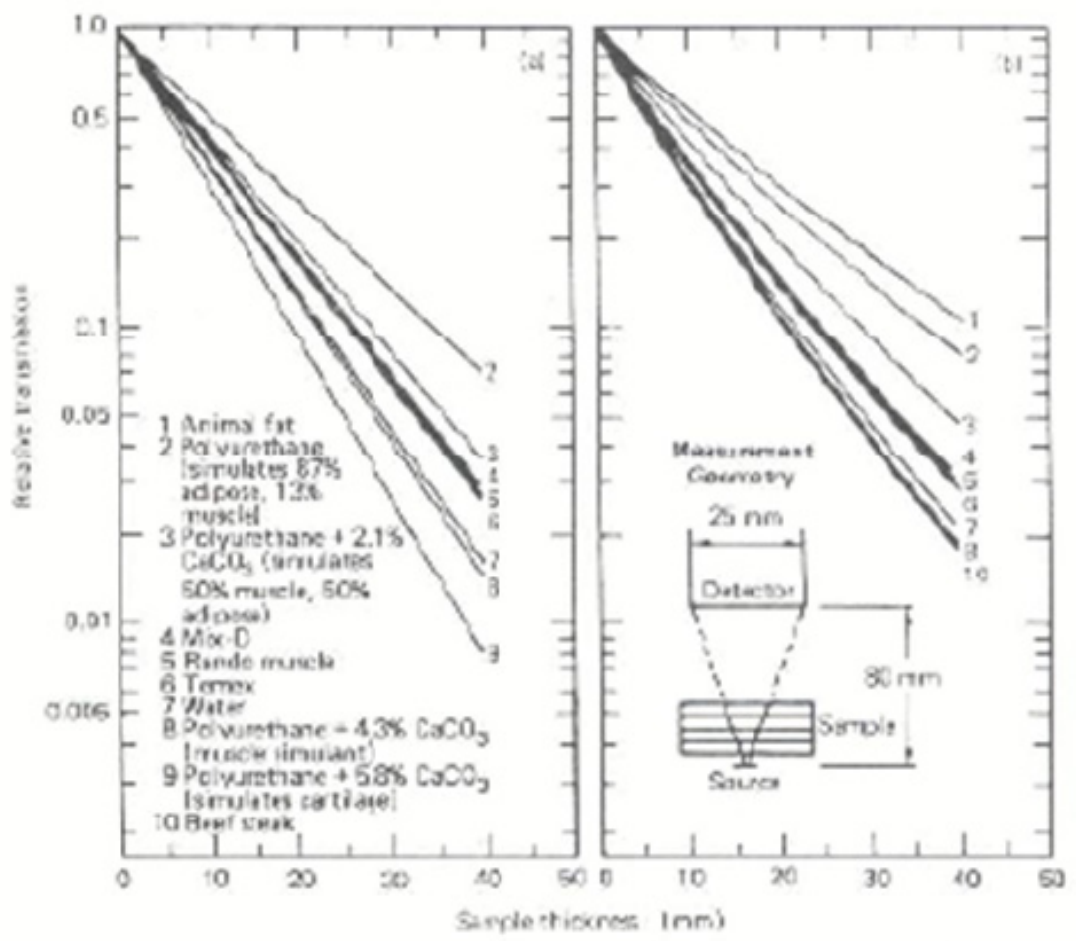

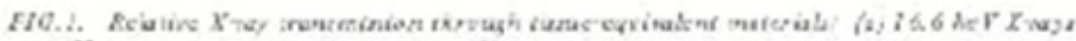

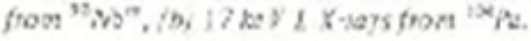

by bone and soft tissue, untertaistes in estimate of internal deposition petterm, and bx permissible ongan burdens (foe exasaple, $16 n \mathrm{Ci}(502 \mathrm{~Bq})^{23 \%} \mathrm{Pu}$ in lung) make detectien and quanhfication of transtaric elerreats at health-protection Isvels difficals.

A key aspect of the in vivo assay probleas is the need for a sealistic pbantom thst (a) reprodtchly sirulites the count

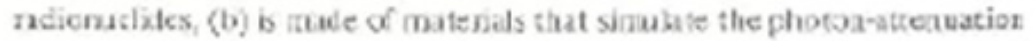
fropartias of humun tisure (mascb, boat, adjipose tisswe, cartiage sond luag) at ereiges beiow $20 \mathrm{keV}$, and icl is cosed encuah to be axed $\mathrm{n}$ compernt the perfomane of countung systers it a rumber of vitely separeted laboratories.

The fiest of three phertorne has now teen eomplotad at che Lawnence Livesmcre Laberatory (L.L.1) Phantem-const niction criteria are based on requirsmenisestabished by te Linited States Department of Erergy Intercalbratioo Committe for Low Eaerzy Ptutoa Messureurents [1]. This conmittes is cotrposed of whele body ceunting specialists frem hrge rae Vationsl Laboracory, 


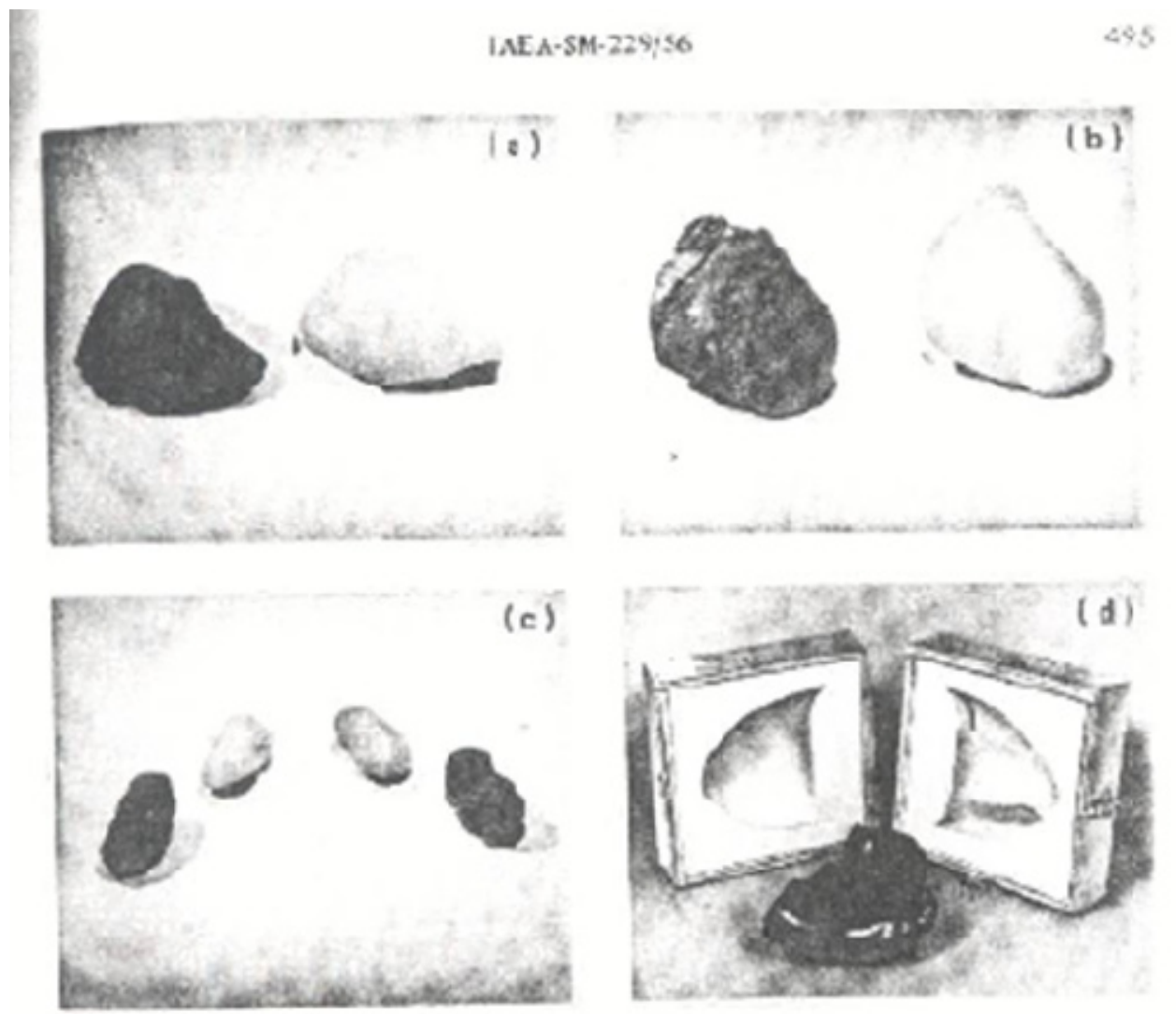

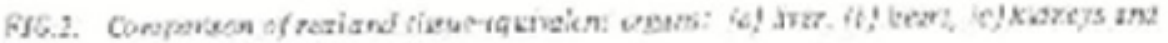
(d) ienx wiri mowh and for rastion.

Battele Pacfie Northwest Lahoratories. Likzcnac Livmose Labo:alory, Los Alamos Scientific Laboratory, Wound Liberatory (Nenzato), Roely Flats Plazt IRockwell Interational), asd Sa-anrah Rizer Plan: (Da Dont)

Tha phartern simulates o humur rale torse wothout hod or arss and is ferminared iust above tie petris The stature ts litat of a nat $1.77 \mathrm{to}$ tall, weigh ing 76 kg. The phanton ccosists of a tissue-etja bacat CTEl pelsurethare tcrso shell with a human make ri cage znkdadid terein. Tiwiesquivelent lunst, heart. liver, kidneys and spler, wishadditional IL material sinukarb intestmes and body nuids, fll the pheatern alionimal eavity. Ke have made chest phtes of 'TF materia! that can te ore:laid on tic phanion te sinulate tie

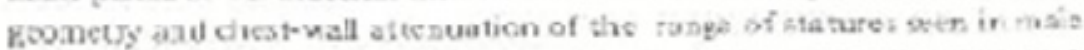
radiation workers. Two scts of ehest plates zare been mate tn crier lo sianatate

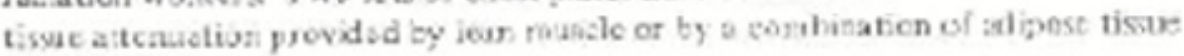

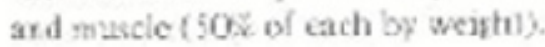




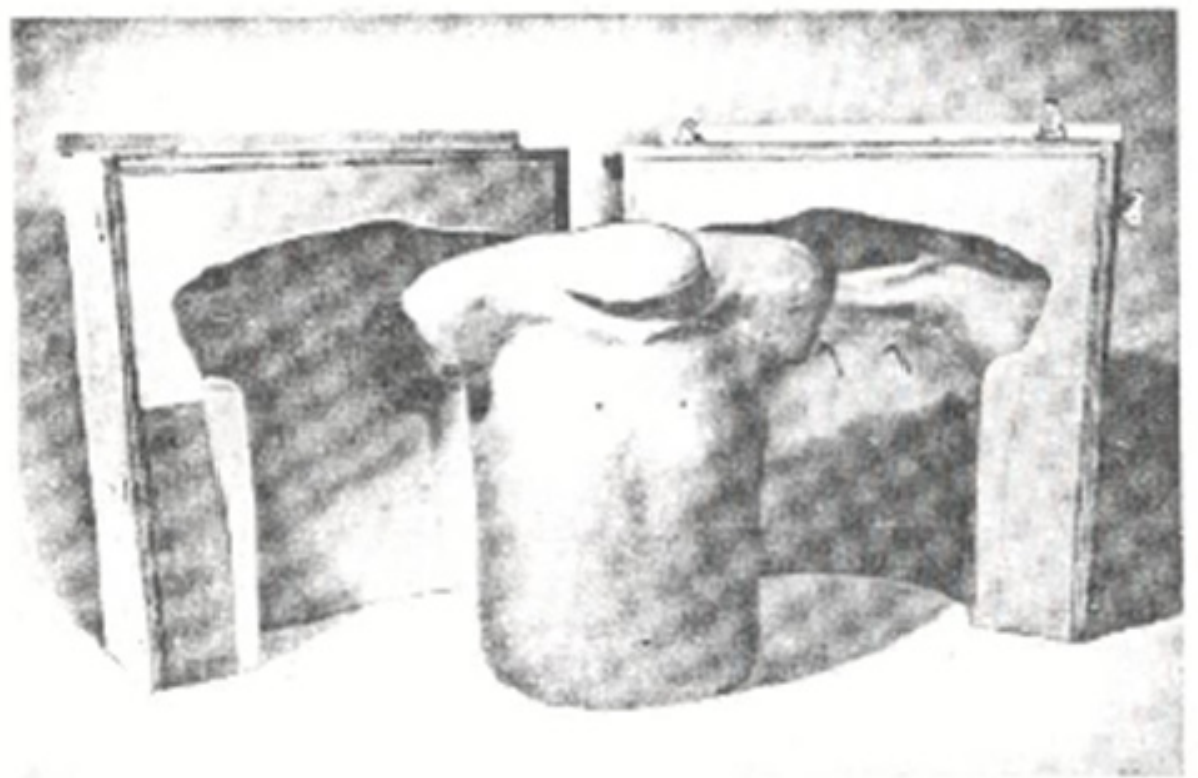

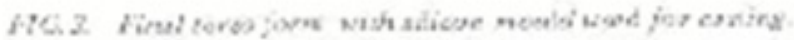
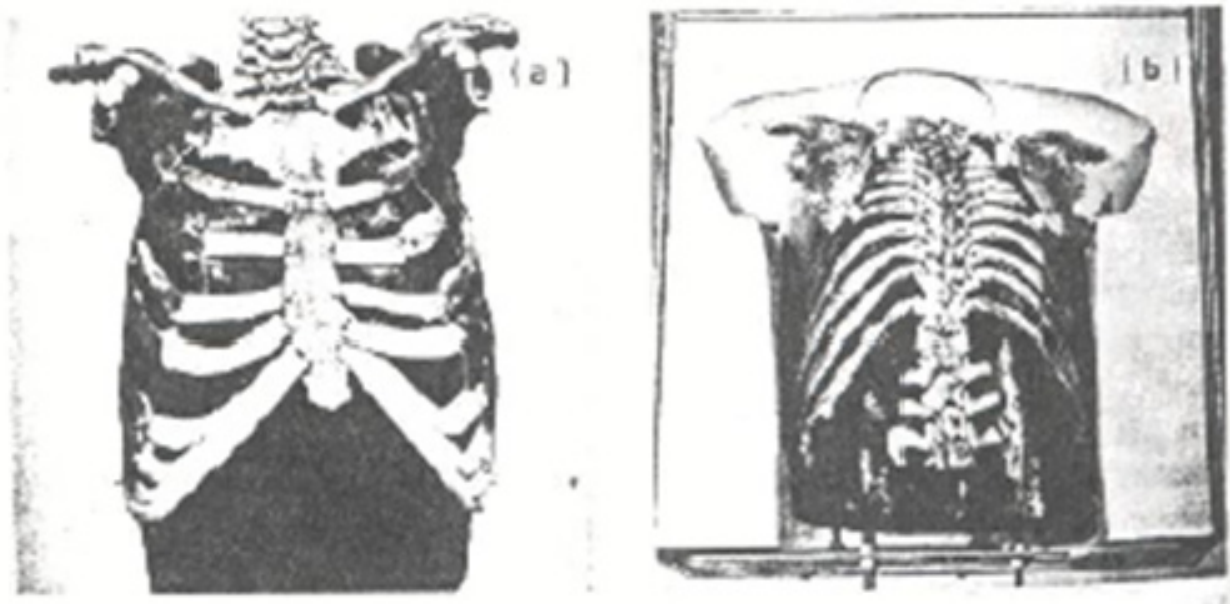

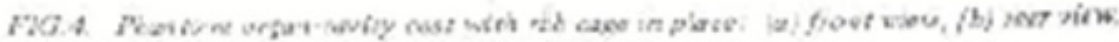

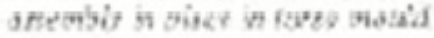



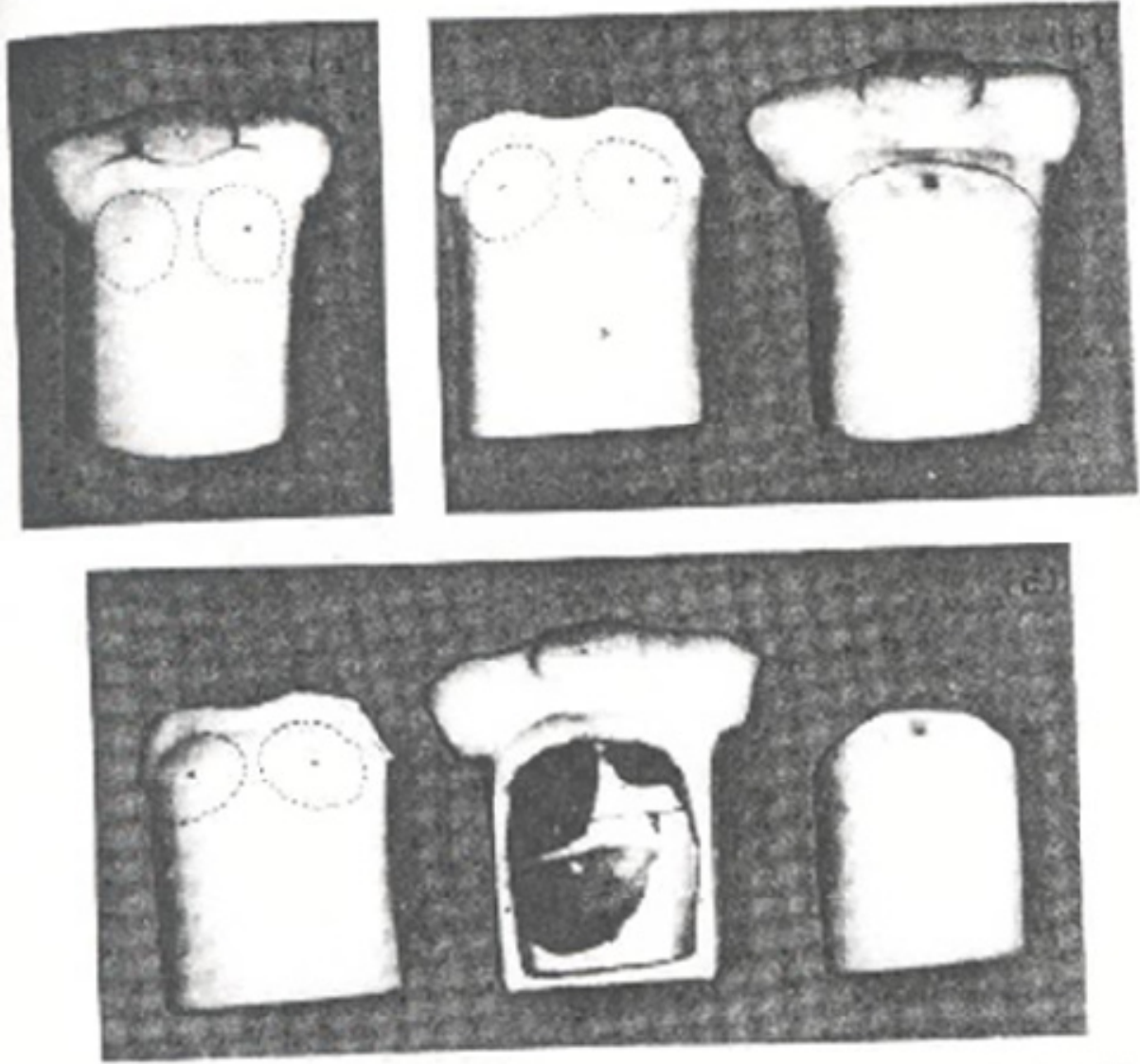

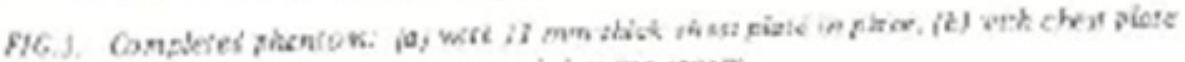

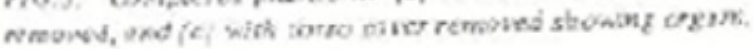

Unform deposition of tranuranic so topes is an isciviousl o:gan can be sroudated by keteling the TE cryas with the cppropriate materials We he aow medo sots of lungs and lyaph nodes that are each labelled with highly pure

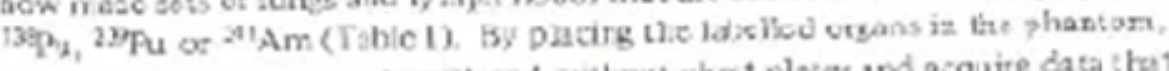
se car make measurements $\mathrm{x}$.h and without chest ploses end acquire data tha1 caa te used to develop counter cajorstios curves far cach of the isctopes of

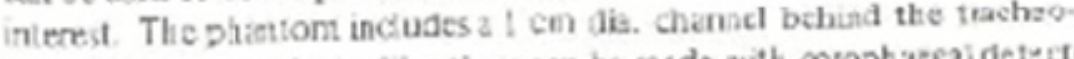
broacial plone so that calihatiers can be made with ocsophageaidetectors.

\section{TISSUEEQUVALENT MATERIAL}

Radiologits and whete-bojy-zouritae specaliss tuve axd varjous material: to simulate tissit. Th: sciection inclucte, readly avalatie natcrils such as 


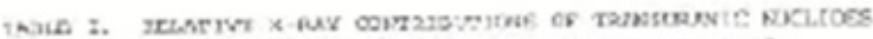

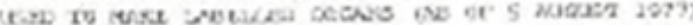

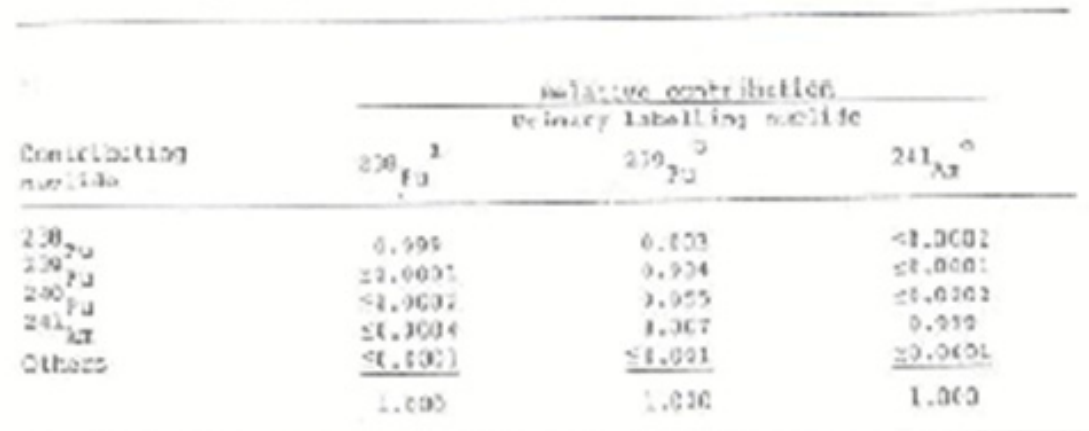

Lof aipta potan-heigint stalyeae.

by ness spescorescy.

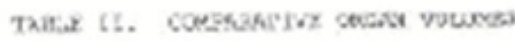

\begin{tabular}{|c|c|c|}
\hline \multirow[b]{2}{*}{ necas } & \multicolumn{2}{|c|}{ Woluas $\cos ^{3}$ ? } \\
\hline & Retresen Min? & Practecn \\
\hline \multicolumn{3}{|l|}{ texps } \\
\hline Cet: & 2152 & 16a \\
\hline a: at: & $2: 53$ & 2181 \\
\hline retal & 201: & $3(6)$ \\
\hline Inste & 243 & Tar \\
\hline G:ver & 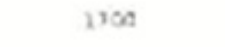 & 2050 \\
\hline $\begin{array}{c}\text { Kiuley } \\
\text { sete }\end{array}$ & $149^{\mathrm{b}}$ & Lr \\
\hline aight & & (9) \\
\hline$\$ ; 1: e n$ & $2 \pi$ & 15: \\
\hline
\end{tabular}

3asec on thed teer. nat. 98 .

giszipotion sotwor lest and ticht kianers rot ase.

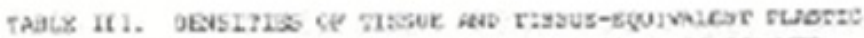

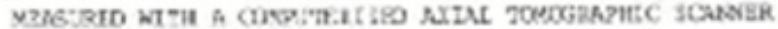

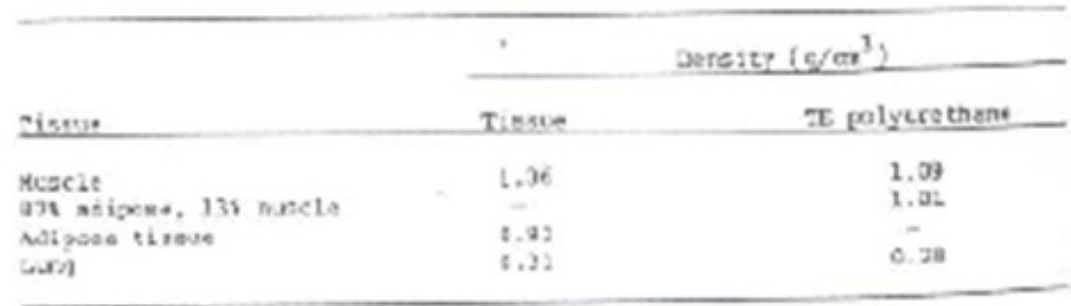


Luelte, Perspex, Presjwood. pelyethylere, and even water.' Hovever, when

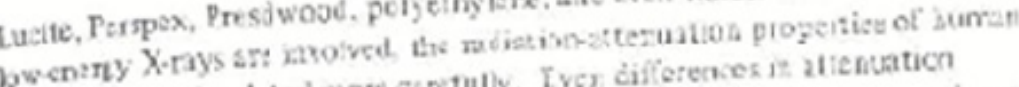
Gste nusi te simulated more carctully. Ives differences it altenuaticn

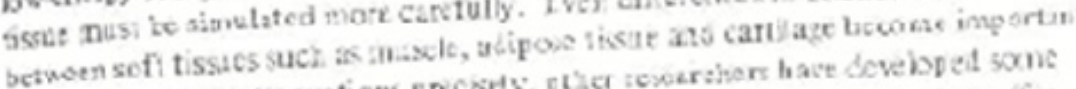

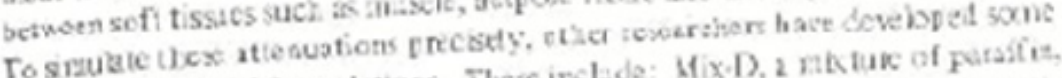

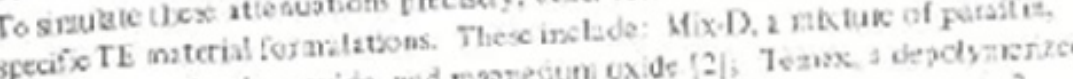

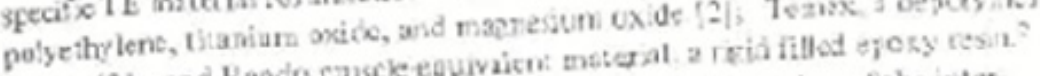

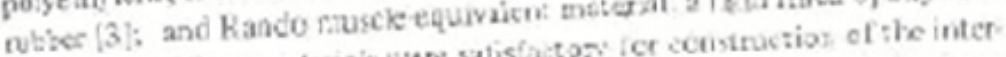

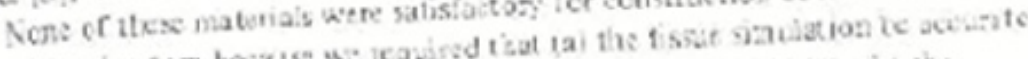

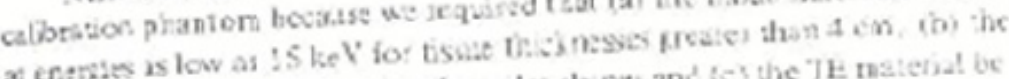

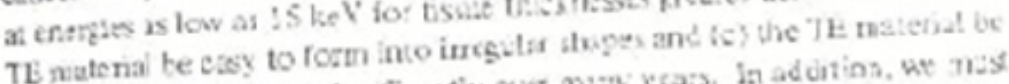

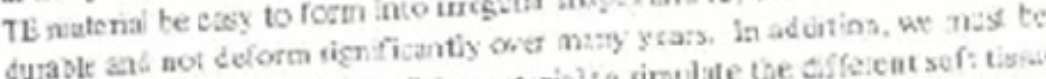
the :ovar the compestion of the materialso simulate the ciffecent sof: tistues -

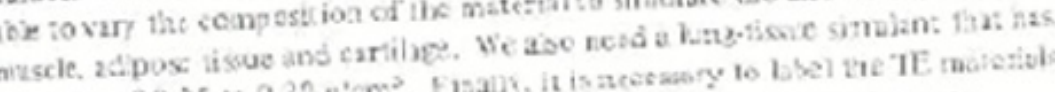

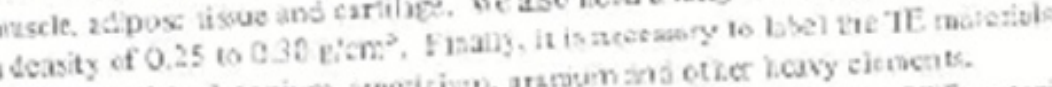

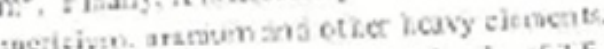

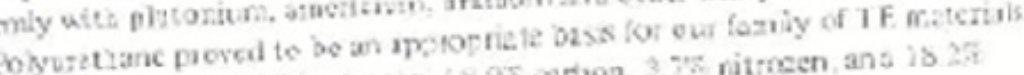

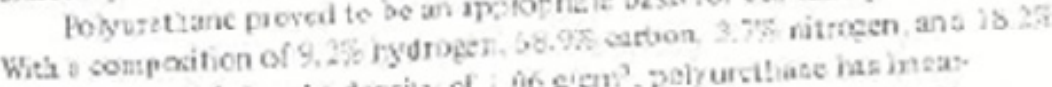

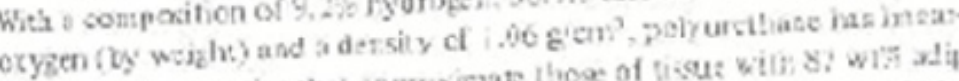

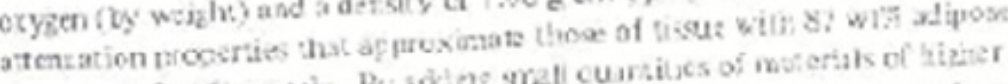

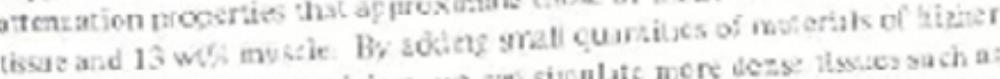

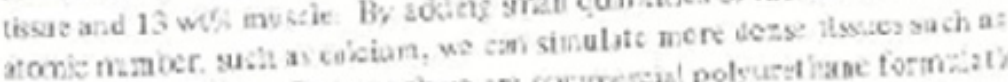

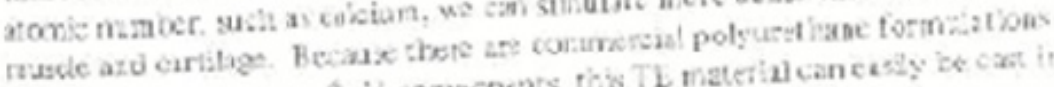
that inrolve maxtme two fiuid components this TL material cancesily be cast it

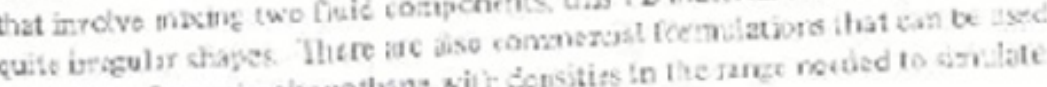

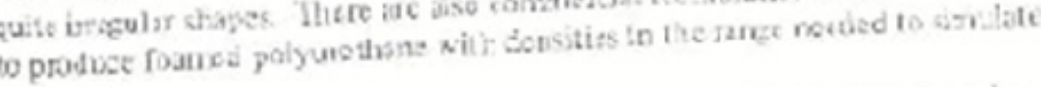
vag tzsa.

Aithoun ve hre xic:ted cae sonnercial pelyarcture Scotchiast b?

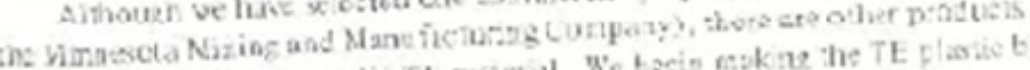

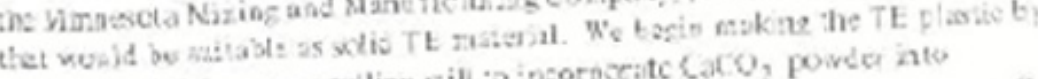

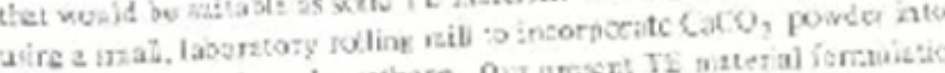

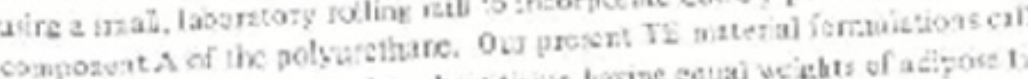

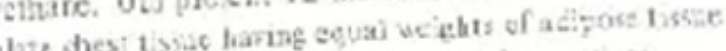
for $21 \mathrm{ks} \mathrm{CaCO}_{3}$ to sinculate thesi and macle, 4.2 wit $\mathrm{CaCO}_{3}$ for enculation.

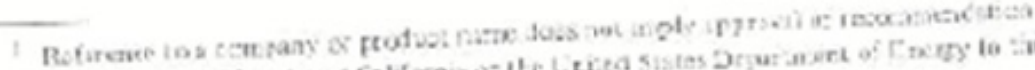

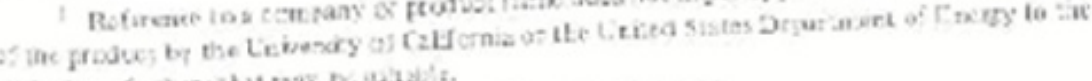

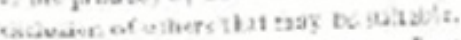

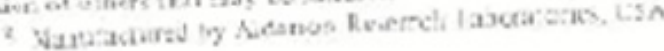


OLr rocmulaten of the tung-equivient materal abe insclsese two-canpentent pdyucethanc, $1940 D$ (blsck), avalabie tron the CPR Jivisoa of Upjohn Corperntoon. A small quantity of water is added to nonerate fouming. The

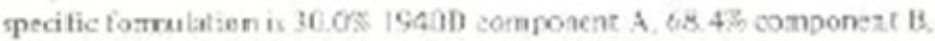

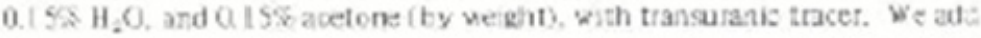

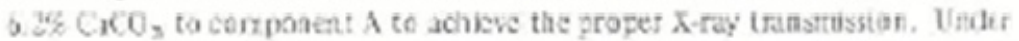

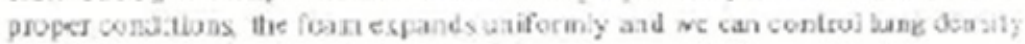

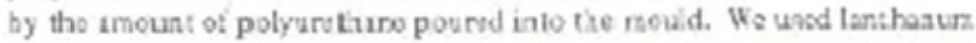
nitm te cariar for the tuaneranic nuclides to achieve uniform distribation $\alpha^{2}$ the ratooctive naterial in the model lunss $X$-ay Gicrescence measurenents or the lanturum coscontration thowed that the carrier dis ribution in sarole lungs was uniferm to wishia $\pm 4 / C$ of the average value.

Wo have ressured the photon transmissions of varioas tosues and TF

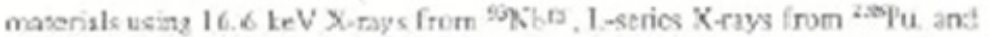

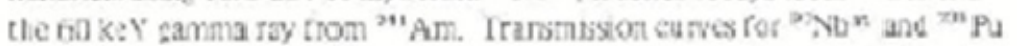

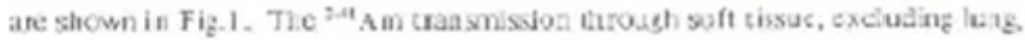
hus a very zurow soresd, through $4.0 \mathrm{~mm}$ of semple, $63.0 \%$ for zturela and muscle equivient polyurethase to $66,7 \%$, for adipose tistu and unloated

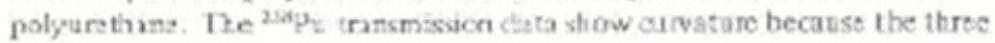

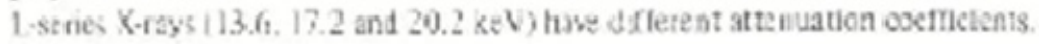

\section{FABRICATION TLCIINIOUES}

The tonss and organ moalds are all based on solid pliser esstz made from a naie culaver prosided by; tze Anatony Departacat at tie Chivenity of

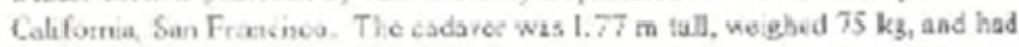
a chest aircuraforenoe of $1 . B 1 \mathrm{~m}$. In esmparion, a random sample of 500 nale LLL and Los Alarnos Saentitic Labaratory empiofscs on the arange wete $1.7 \mathrm{~m}$ talt, weighed $75 \mathrm{~kg}$. and has a chest cincus ference of $2 \mathrm{~m}$. Theorgars and TE repliess ase altown in Fic 2, Orgaatcplica volumes are preveted in Tabla [1, torether with valuss for Reference $M$ fan $[4]$.

An Impertan I part of the plantorn coastruction was racditication of the torso casts to that the phanter shest wal averlying the largs would be an thin at poscible while still accom modating the rit cage. (This alous us to messur? arteacation for tat witest range of chest-wall thicknesses) we made a thi jlislie zeprocuctien of the chest exterior by vacuurn forzaing a plastic theet on tue ploster torso cest. Hic merked this chast plato with a grid, drilbed heles at the gaid poiris, ind placent the plate cver a cast of the crran cavity. Fron ultrasoricsecan da ta takezi on the chests and ahdomens of a laree croup of radiation workers and from publisted anatorizal data [5], avo labrlated the thiracsi te alstic prories of chest-wall and Abuonimal-wall thickncsess for the 

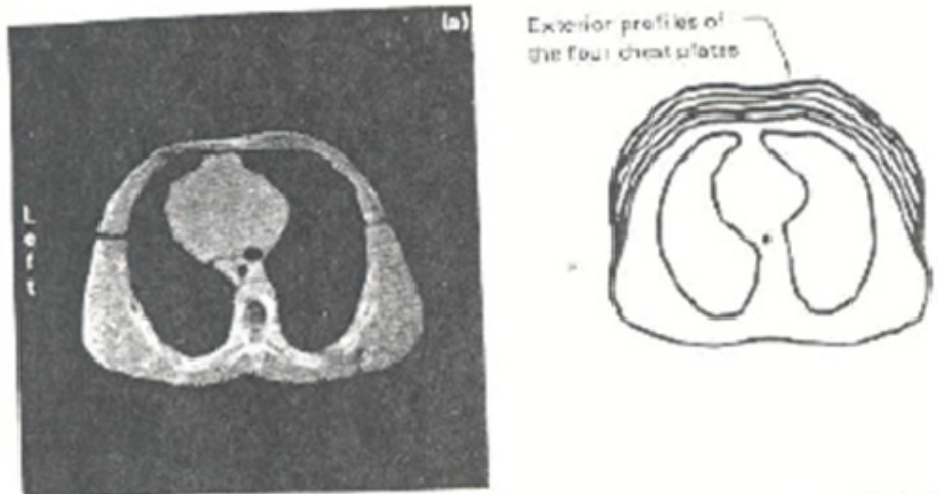

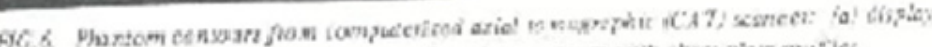

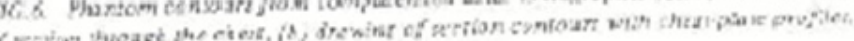

fhericm With the Dlaslic chest plate in plac: on tic cogat-corily ejat, wet nepped in sctual chest and abdom inal-wall spacings. The differenos bet ween

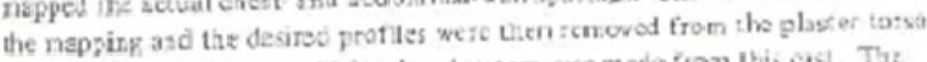
cat. A siticonrubber moald for the phant or wes mate fron iticest. The fira) tarso molel is shown in Fe. 3 togetier wilh its movid.

After romovel from the eadaver, the rib cage was clcanted to renor: sofi

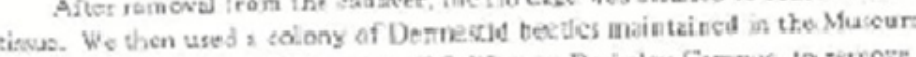

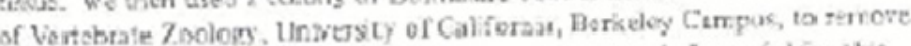

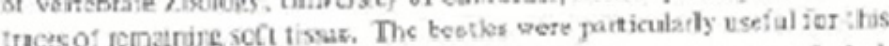
pirpose because they have access to sabal volos in treskeletor (particulaty in tbe veretrac) bul do not altack cartilag. The cartilate was thes seved jor future use. Finally, we hed ammonis and a rapoun sokent to degrocas the bartes. We

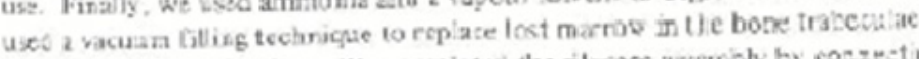
with Yix-D tissue smulant. We cuncketed the zibcapo assembly by conzect ing verctare with nyler pine and the fibs with aylon striag. Special xecticas of polywethane cartiase-simulant connest the ibs the stoms. The rib eage is

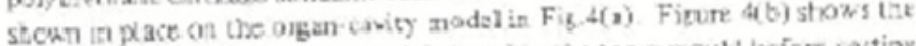

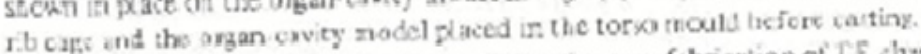

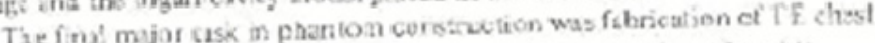

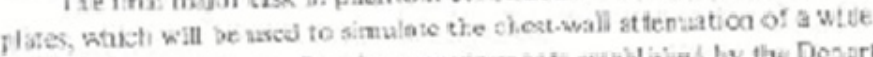
verie:y of mie staturex. Based on zecurements establisied by the Deportenezt

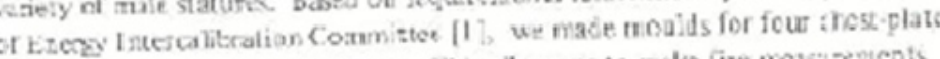

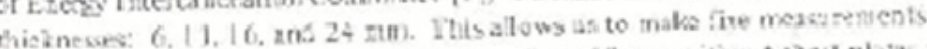

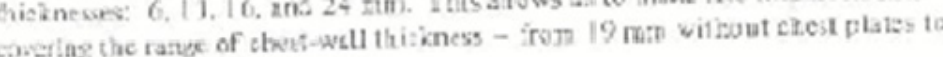




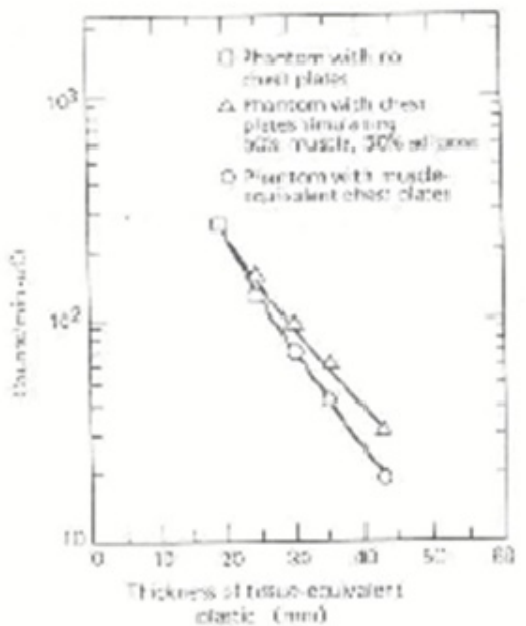

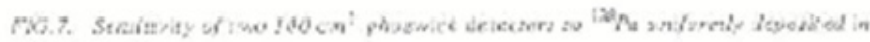
grectere Ang

43 mem with the thickest platce. This ranee incudes more than $95 \%$ of the male radusion worters anceitord in the USA Two sets of plates bave now beca cat: ase with muscte-cquirelent astorial and ane with polyurethane to siriula:e tissae composed of $5 \% \%$ masde and $50 \%$ adipore (issu. Figue $5(2)$ show the phantom asembled with tiue 1 I mun thick chest plute in place. Fisure s(0) saow the phantor. and chest plate apart, anc Fiy.5(c) shows the phas tan wi th bota lies chest glate and tarso cover reizoved.

\section{PILVTOM EVALUTIONS}

Tie sucesss of the phantem ie an isterualibation progran mo dopends on how faithfilly it sirulatas human morpholosy azd radistion transnistion of human tissre. We used a cornpuerized axial tomographic (CA [) seanacr at a jecal hospital to help evaluare titese qualities. The CAT scanaer yelds excellent norphoblical da:a tuough cross-sectoral wass. 1t aso provides dete on the

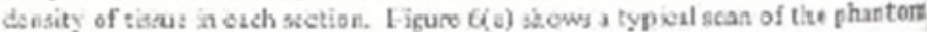
tasough tive lang region. Th'o sshe matis in Fig.s(b) shows contoses of a typical section of tire phuntosn chest and the relatire profiles of the four chest plates Conpurative tisse aenstes neasured with the CAT scaner tor typical bunan 
and pruntorn tissues ane shown in Fabe I1. The CAT sasner dats diepents on

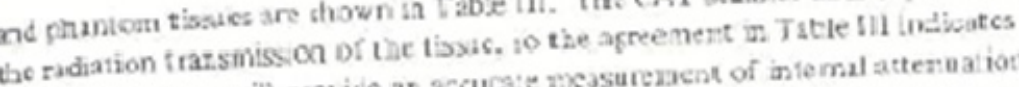
that the phasion will provitc an accura:e measureacref of antemal atternation.

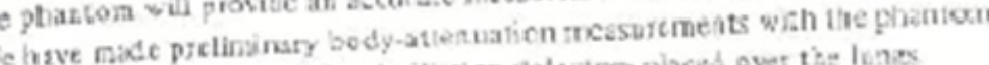
dia. phoswich scistilwion actectors plased over the lunes

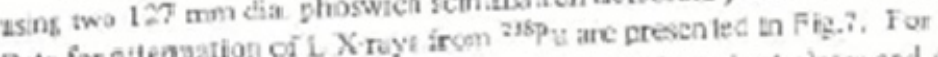

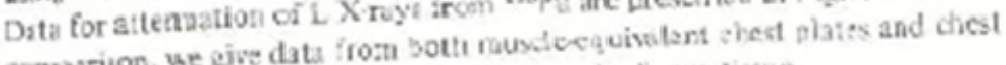

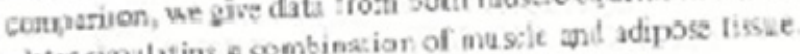
phates sinulatinz combinat cor

\section{ACKNONLLUCEMENTS}

The authors wish to tsenl Harry P. Conad, Norzaca W. Beye: ayg. Steve W. Wizchester, eqd atl the personel in the L.L otastie

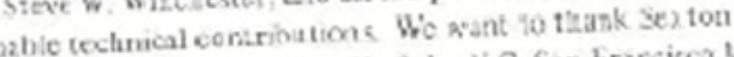
siop for their valuabie teche Jery Rutch ic, W of the L,C. Sin Francisca Medical

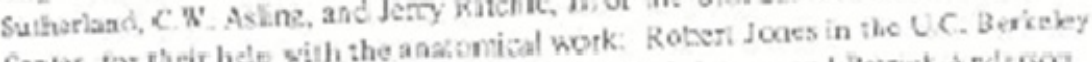

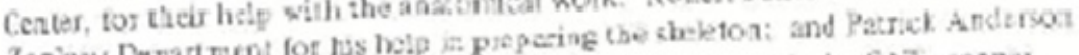

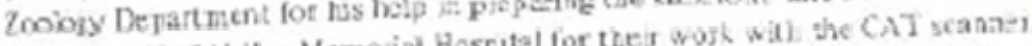

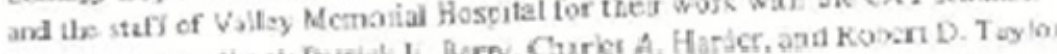

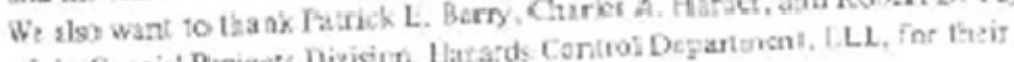

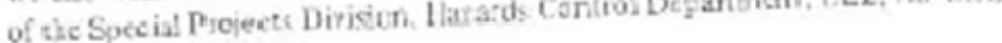
centirains support.

\section{REF EREYCES}

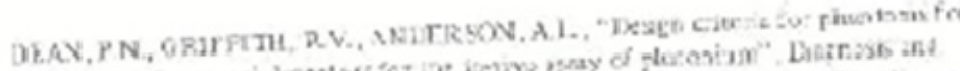

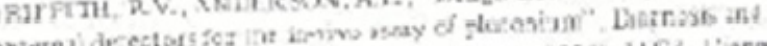

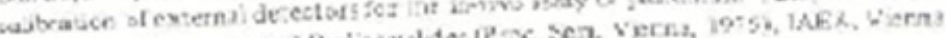

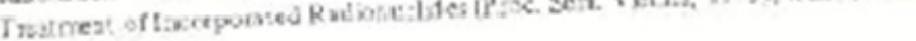
(1976) 265.

[2] JONES, D.E.A. Ex,S.R=Liul $32(3959) 66$.

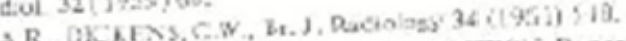

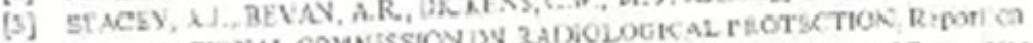

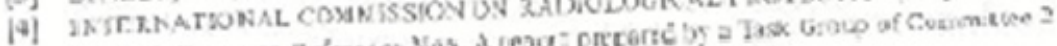

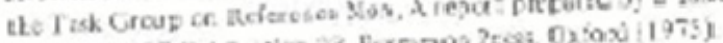

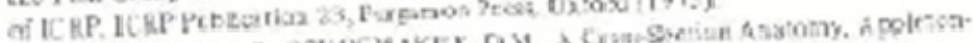

(3) BYCLESITUAR, A.C., SCHOEXAKE.

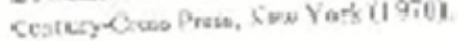

\section{DISCUSSION}

1. ORLRHOFFR: I axc two Guestons rgerdieg your timucethivels

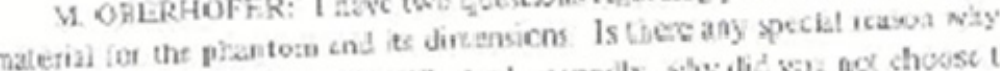

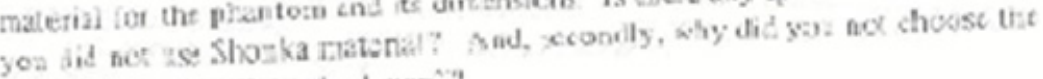
fin atsions oi the "standard ran" 
R. V, GRIFFITH: in ansece 10 your tisst quastion, we aceded a material frem which :t wevid be easy to maks ienegular chapes such as those of ta: forso shell and orgars. Also, we manted a maccrial whose composition could exsly te changed to sigulate ine fadial ton attenualion propertes of cisacs othes tha: nualt. Fisally, the retzed of incorporstion of the ndianuekibs in the creass la ad ta be fainly simple. The Sherica plastic does not meet these nequiremens.

Wit did not use "stuedard max" duneeswons becaux the cyplad worker was fourd feum our studjes at Les Alsmos snd the Lawience Livemoce Laberetory to bs romexiat large. $\boldsymbol{A}$ "stendend ana" phantom would then have bsen. smaljer thas the aversge person Ne vinted to simalat:

D. COULSTON: What is the cost and potential avalabilisy of the planton destibed?

R.V. GRIFPTH A very ronghestimate of sust based on the labors nuolved is $\$ 125000$ The natcrisi eoses are incidental. The cost of future phantons would, of ours, be nuch less, as re now have all the moalds reccessery for the castiry.

Ne do not iniead to niss-preciuce taese plantoms und fare not yct doci.ted whut te du with tie anoulds when the last one is finicked. However, anyoze usirg the rnoulds would have to face the practical problem of obtaining the rib case. The phantorns will be used in an interconpansun prograne sporsered by the Untied States Departanent of Erergy. The protocul lor the fist roand of the progromme aas nov boes eatablizhed. Huwever, after that part of the work has been eonglated, it is quite goasible that intorcompanieas atadies could te extended to other çualificd laboratorics.

P. KOTR.PPA: I should like to sut a eereral question. We haye bead

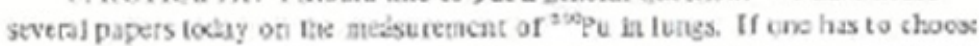
between different de teetion sy $\times$ eem, which sysem do you thirk would give the best performance for tie inwestment? Also, what is the minimum ${ }^{23}$ Pu that can bedetected is the lungs of a atandard mas at the prestnt time?

R. V. GRIFFTH: To my nind the phosvich or dual sant:latios detecto: is both the cheapes, and most sensitive devioe availstle for ${ }^{34}$ Pu lung souating. The situstion could of courso change in two or there your.

CI. URBR R.FFR: The trosd in the Linited States of A merica and the Lniled Kirgdon is to ze phoswich detectors for the amulysis of $22 \mathrm{Pu}$ in the lune On tie basis of most sersitivity for the dollar. this system wculd seen to be the bes available to tace. On tac cusstion of detection linits for ${ }^{2} \mathrm{Pu}$ in the laxe. ne tend te zroid stating a univessal couz ting lower tanit. Howavor, our opinos at Leis Alamos a chat, in the present state of the art, only approximutely one permissible lung burter ( $16 \mathrm{aCt}$ ) is detectable in a standard-sized worker with a single soust. 
and phantout tissues are shown in Tabte t11. The CAT wasner dats depants on the radiation frazsniss on of Lae tissac, so the agreemert in Tatle fll indicatcs that the phaston will providc an accura:e measurement of antemal atternation.

Wie have nide prclinsinary bedy-attenuation rocsurtmeats wish the phentom

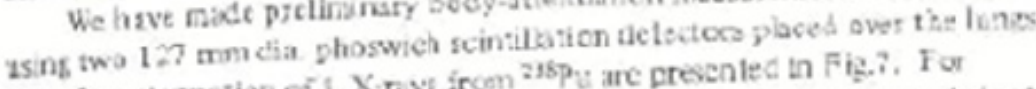

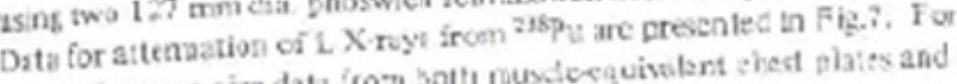
comparion, we give data from both ruysferequivelent shest plates and chest phtes sonslating c combinstion of mus:it and adipose tisse

\section{ACKNOWLLUCEMENTS}

The authors wish to tsanl. Harry P. Conra, Norzan W. Boyez fulor. Steve W. Wirchester, eta all tho perionel in the L.L plastiv kabable tochnical centributions. We sant to tank Seztoti

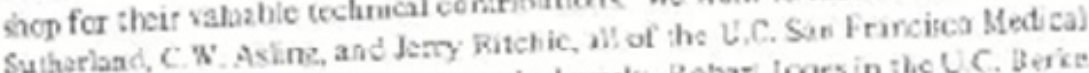

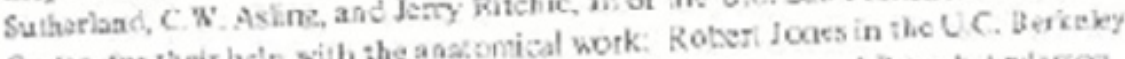

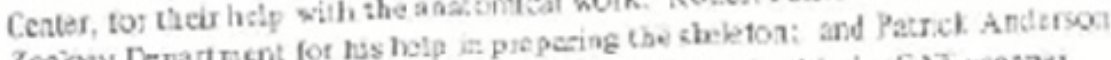
Zociony Departncnt for hes Hetp Hescital for trei work wil the CNI seanas

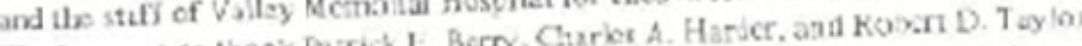

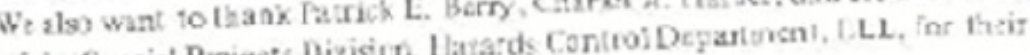
centiruing suport.

\section{REFERENCES}

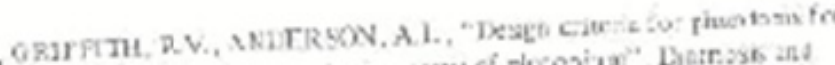

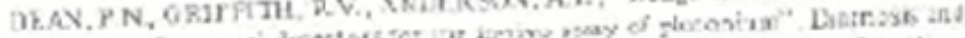

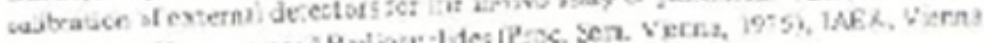
Trestreat ort: (1976) 262

[2] JONES, D.E.A. Bt, S. Rid.ul $32(3959) 65$.

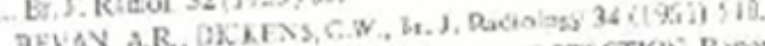

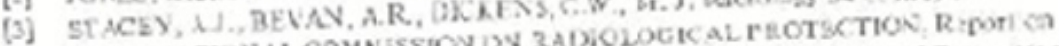

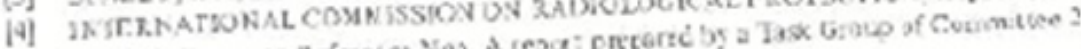

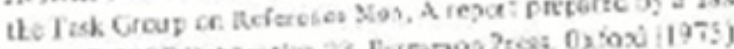

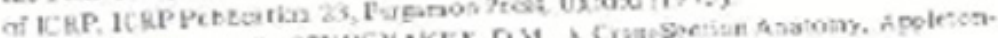

(5) BYCLESIYYARR, ג.C.,SCHCEXIAKI.

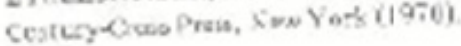

\section{uscussiov}

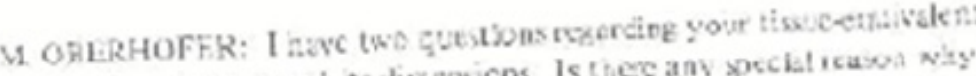

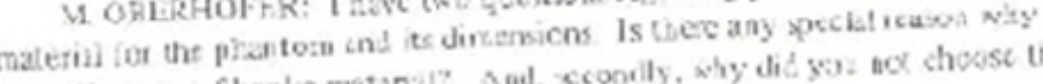

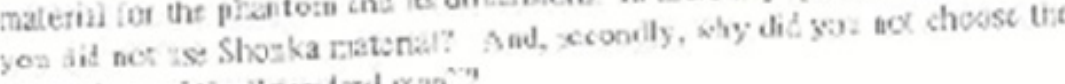
cincasions of the "sindard ran" 


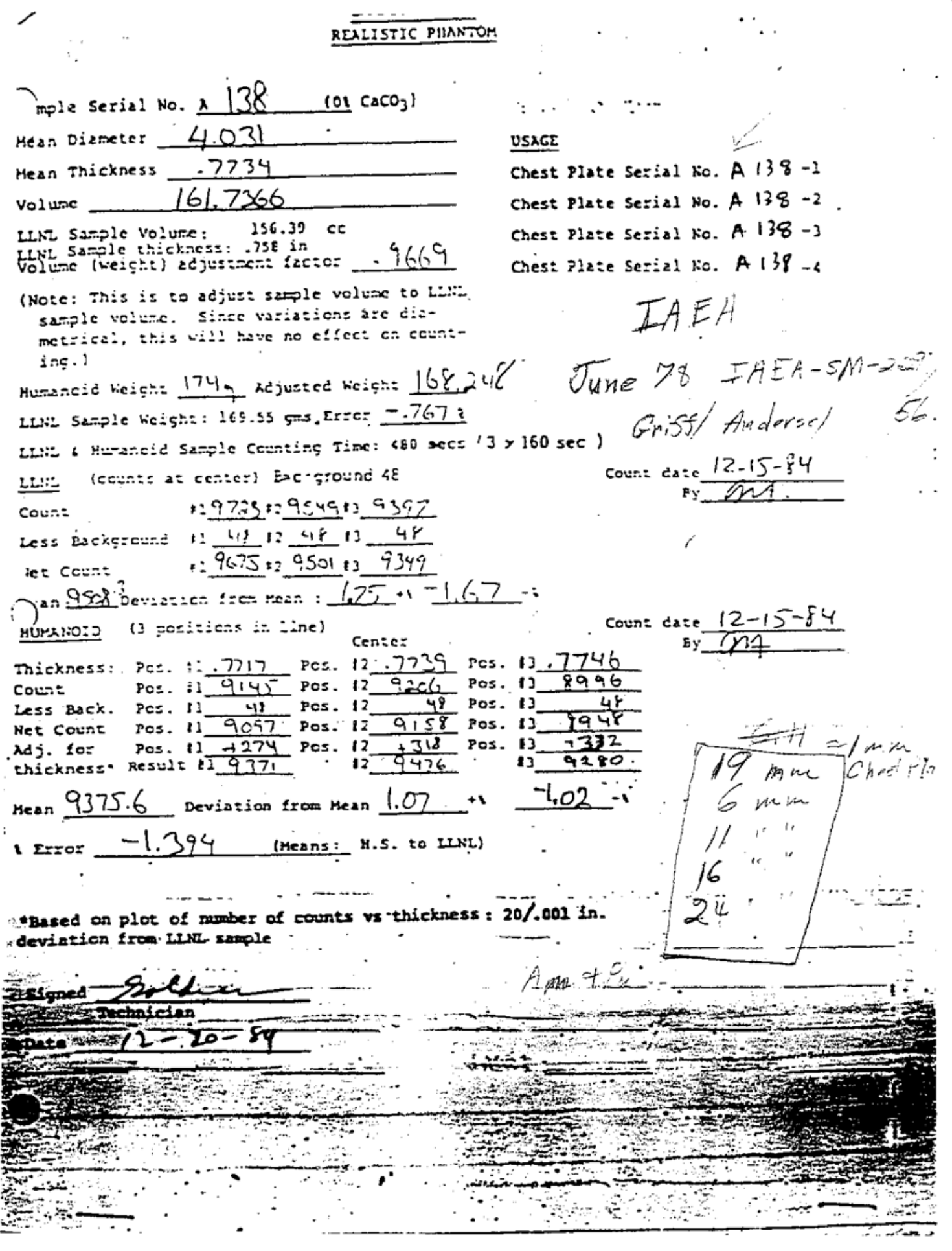


R, V, GRIFFITH: In ensece 10 your tirst quastion, we aceded a material frem which at weuid be easy to make ienegular chapes such as those of ta: forso shell and ozars. Also, we wanted a macrial whose composition could easly te chenged to sindate ine radialion st te nual ion propertes of ciseucs othes than ansalt. Fizally, the retzed of accoporation of the radionuckides in the creans

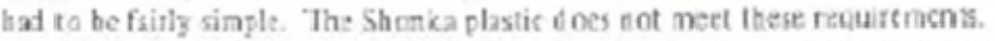

We did not us "stundard max" dunetsoons becaue the typlial worker was fourd feom our studjes at Les Alamos and the Lawieace Livemore Laboratory to be yonexiat large. $A$ "zlesjunl ana" phantom would then have bsen in.aller than the average person No wanted to sirmalate.

DJ. COULSTON: what is the oost and potential avalability of the plaaton descibed?

R.V. GRIFFITH: A very rough catimate of ceot based on tho labour involved is $\$ 125000$ The watcrist meses are incidental. The cost of future phantons woutd, of course, be nuct less, as ne now have all the monlds reccossery for the casting

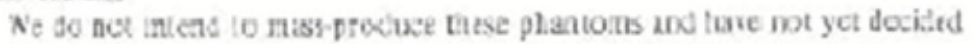
whet to du with tie anoulds when the last one is finiaced. However, anyoze usirg the rnouids would have to face the practical problem of obtaining th: rib caze. The phantoms will be uxed in an intercompansoz. programne sporsered by the Untiod States Department of Energy. The protocol lor the first roand of the prograsme has nov been catablistud. Huwaver, after that purt of the work has bacn complated, it is quite goasible that intorcompaniose atudies could ke entendad to other cualified laberatorics.

P. KOTRAPPK: I should like to sut a eerreral guestion. We have beard

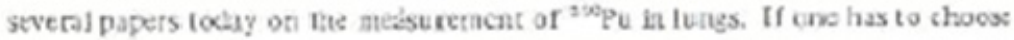
betwecn differeat de lecticn sy $x \in e m$, which sysem do you thirk would give the best performance for the investment? Also. what is the miainum ${ }^{2}$ Pu that can bedetected is the lungs of a atardard mas at the prestnt tims?

RV. GRIFFTH: To my nind, the phoswich or dual santslatios detector is both the cheapes. and most sensitive devioe availatle for ${ }^{23} \mathrm{Pu}$ lung souating. The situstion could of courso change in two or thre years.

C. I. URBBARGFR: The trond in the Linited States of A merica and the Lnited Kizgd on is to ase phoswich detectors for the ambsis of ${ }^{22} \mathrm{Pu}$ in the lung On tie basis of most sersitivity for the dedtar, this system would seen to be the best 2vailabie to tace. On tze cusstion of detection linits for ${ }^{20} \mathrm{Pu}$ in the laxs. ne tend te aroid statigg a univessal couz ting lower limit. Howror, our opiajoa at Les Namos is chat, in the present state of the aut, only opprosimately ore permisibla lung burder ( $~ 16 \mathrm{aCt}$ ) is detectable in a standard-gized worker with a single sount. 


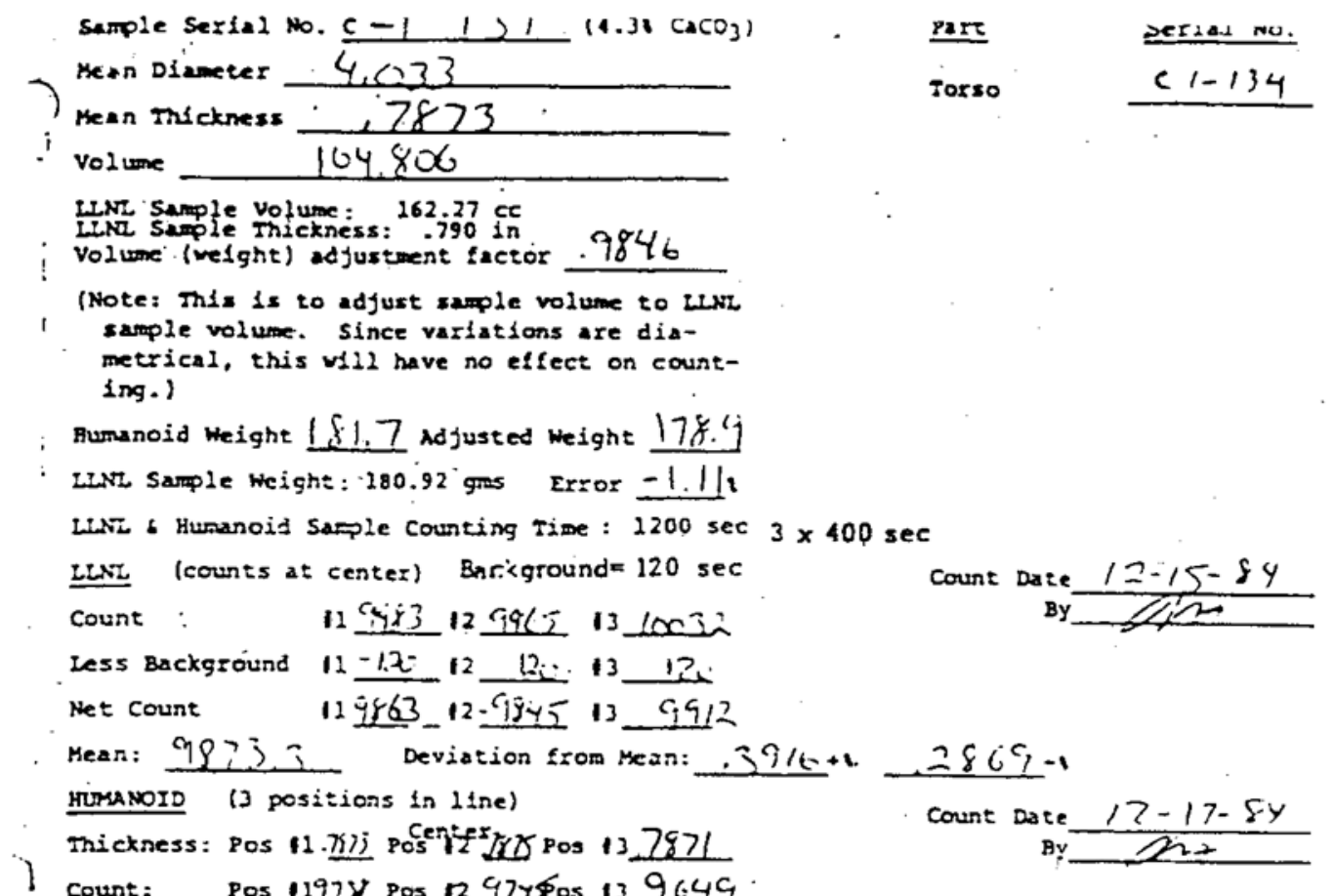

Count: Pos 1197y Pos 1297 95os 139649

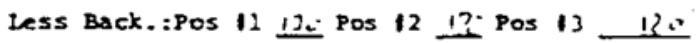

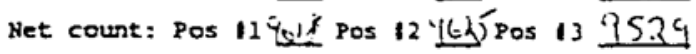

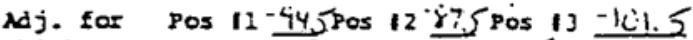

- thickmess- Result 11922 - 129537439427.5

Mean 9496.16 Deviation fram Mean $+425 \%,-.723 \%$

IError $-3.8 \%$ (Hean : H.s. to LLNI)

-Based on plot of number of counts vs thickness: $35 / .001$ in devistion from Irst anple.

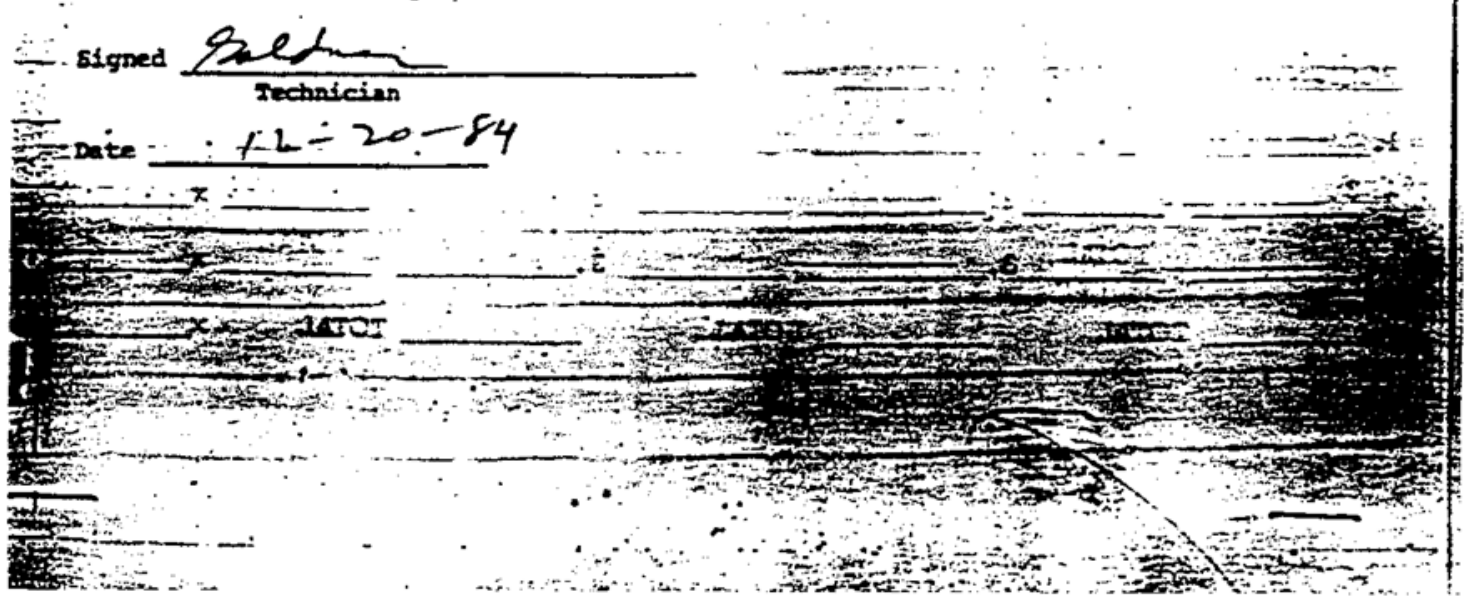




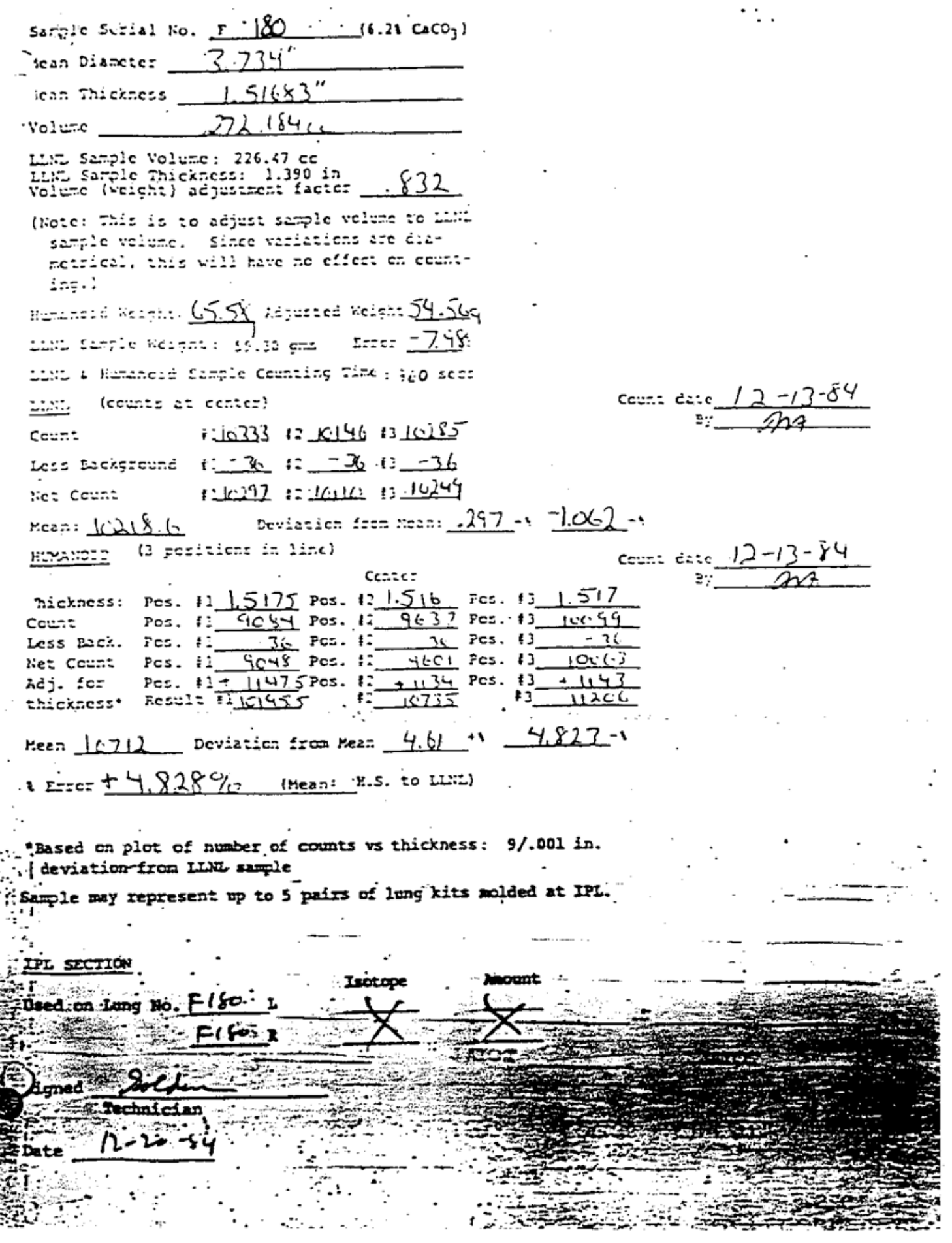


Grid Mortis

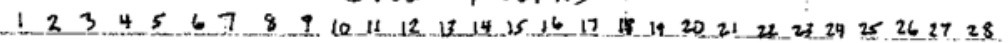

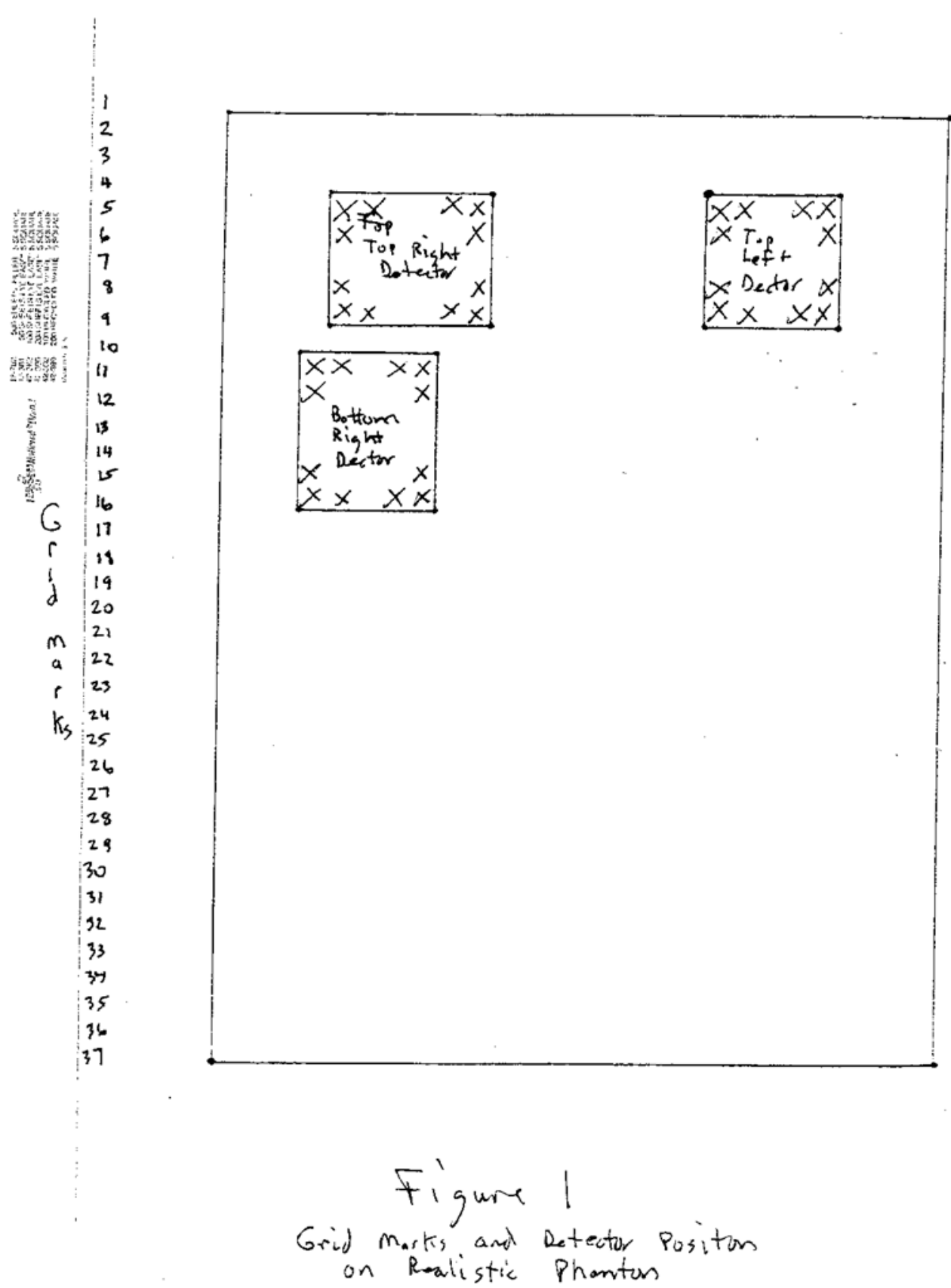


Table i

Cl-134, Right Side

\begin{tabular}{|l|l|l|l|l|l|l|l|}
\hline & col. 5 & col. 6 & Col. 7 & col. 8 & Col. 9 & col. 10 & Col. 11 \\
\hline row 4 & XXXXXX & XXXXXX & $16.24 \mathrm{~mm}$ & $16.63 \mathrm{~mm}$ & $15.58 \mathrm{~mm}$ & XXXXXX & XXXXXX \\
\hline row 5 & XXXXXX & $16.65 \mathrm{~mm}$ & $15.17 \mathrm{~mm}$ & $14.59 \mathrm{~mm}$ & $14.71 \mathrm{~mm}$ & $14.54 \mathrm{~mm}$ & XXXXXX \\
\hline row 6 & $16.12 \mathrm{~mm}$ & $14.55 \mathrm{~mm}$ & $14.46 \mathrm{~mm}$ & $13.28 \mathrm{~mm}$ & $13.54 \mathrm{~mm}$ & $13.41 \mathrm{~mm}$ & $12.70 \mathrm{~mm}$ \\
\hline row 7 & $15.24 \mathrm{~mm}$ & $13.23 \mathrm{~mm}$ & $12.17 \mathrm{~mm}$ & $12.90 \mathrm{~mm}$ & $12.95 \mathrm{~mm}$ & $12.88 \mathrm{~mm}$ & $12.72 \mathrm{~mm}$ \\
\hline row 8 & XXXXXX & $11.71 \mathrm{~mm}$ & $12.65 \mathrm{~mm}$ & $12.12 \mathrm{~mm}$ & $12.62 \mathrm{~mm}$ & $12.75 \mathrm{~mm}$ & XXXXXX \\
\hline row 9 & XXXXXX & XXXXXX & $13.23 \mathrm{~mm}$ & $12.73 \mathrm{~mm}$ & $12.05 \mathrm{~mm}$ & XXXXXX & XXXXXX \\
\hline
\end{tabular}

Table 2

Cl-134, Right Side

\begin{tabular}{|l|l|l|l|l|l|l|}
\hline & col. 4 & col.5 & Col. 6 & col.7 & col. 8 & col. 9 \\
\hline row 10 & XXXXXXX & XXXXXXX & $13.71 \mathrm{~mm}$ & $13.03 \mathrm{~mm}$ & XXXXXXX & XXXXXXX \\
\hline row 11 & XXXXXXX & $13.20 \mathrm{~mm}$ & $12.54 \mathrm{~mm}$ & $12.14 \mathrm{~mm}$ & $13.21 \mathrm{~mm}$ & XXXXXXX \\
\hline row 12 & $14.50 \mathrm{~mm}$ & $13.71 \mathrm{~mm}$ & $12.77 \mathrm{~mm}$ & $13.33 \mathrm{~mm}$ & $13.33 \mathrm{~mm}$ & $13.94 \mathrm{~mm}$ \\
\hline row 13 & $13.76 \mathrm{~mm}$ & $13.34 \mathrm{~mm}$ & $13.53 \mathrm{~mm}$ & $13.90 \mathrm{~mm}$ & $14.35 \mathrm{~mm}$ & $15.57 \mathrm{~mm}$ \\
\hline row 14 & $13.35 \mathrm{~mm}$ & $13.38 \mathrm{~mm}$ & $13.60 \mathrm{~mm}$ & $14.21 \mathrm{~mm}$ & $16.02 \mathrm{~mm}$ & $15.94 \mathrm{~mm}$ \\
\hline row 15 & XXXXXXX & $13.59 \mathrm{~mm}$ & $12.97 \mathrm{~mm}$ & $15.33 \mathrm{~mm}$ & $16.22 \mathrm{~mm}$ & XXXXXXX \\
\hline row 16 & $\mathrm{XXXXXXX}$ & $\mathrm{XXXXXXX}$ & $13.91 \mathrm{~mm}$ & $14.07 \mathrm{~mm}$ & $\mathrm{XXXXXXX}$ & $\mathrm{XXXXXXX}$ \\
\hline
\end{tabular}


Table 3

C1-134, Left Side

\begin{tabular}{|l|l|l|l|l|l|l|}
\hline & col. 19 & col. 20 & Col. 21 & Coi. 22 & col. 23 & Col. 24 \\
\hline row 4 & XXXXXXX & XXXXXXX & $14.39 \mathrm{~mm}$ & $14.63 \mathrm{~mm}$ & XXXXXXX & XXXXXXX \\
\hline row 5 & XXXXXXX & $11.76 \mathrm{~mm}$ & $12.39 \mathrm{~mm}$ & $12.23 \mathrm{~mm}$ & $12.31 \mathrm{~mm}$ & XXXXXXX \\
\hline row 6 & $14.21 \mathrm{~mm}$ & $11.49 \mathrm{~mm}$ & $11.55 \mathrm{~mm}$ & $11.76 \mathrm{~mm}$ & $12.68 \mathrm{~mm}$ & $14.14 \mathrm{~mm}$ \\
\hline row 7 & $14.09 \mathrm{~mm}$ & $12.72 \mathrm{~mm}$ & $12.09 \mathrm{~mm}$ & $12.15 \mathrm{~mm}$ & $13.56 \mathrm{~mm}$ & $15.17 \mathrm{~mm}$ \\
\hline row 8 & XXXXXXX & $12.97 \mathrm{~mm}$ & $12.55 \mathrm{~mm}$ & $12.00 \mathrm{~mm}$ & $12.47 \mathrm{~mm}$ & XXXXXXX \\
\hline row 9 & XXXXXXX & XXXXXXX & $12.40 \mathrm{~mm}$ & $11.70 \mathrm{~mm}$ & XXXXXXX & XXXXXXX \\
\hline
\end{tabular}

Average . Thickness for $\mathrm{Cl}-134=13.55 \mathrm{~mm}$ 
Table 7

A-138-1, Left Side

\begin{tabular}{|l|l|l|l|l|l|l|}
\hline & col. 19 & col. 20 & Col. 21 & col. 22 & col. 23 & Col. 24 \\
\hline row 4 & XXXXXXX & XXXXXXX & $6.06 \mathrm{~mm}$ & $6.06 \mathrm{~mm}$ & XXXXXXX & XXXXXXX \\
\hline row 5 & XXXXXXX & $6.03 \mathrm{~mm}$ & $6.03 \mathrm{~mm}$ & $6.01 \mathrm{~mm}$ & $6.01 \mathrm{~mm}$ & XXXXXXX \\
\hline row 6 & $5.29 \mathrm{~mm}$ & $5.29 \mathrm{~mm}$ & $5.29 \mathrm{~mm}$ & $5.29 \mathrm{~mm}$ & $5.29 \mathrm{~mm}$ & $5.29 \mathrm{~mm}$ \\
\hline row 7 & $5.08 \mathrm{~mm}$ & $5.08 \mathrm{~mm}$ & $5.08 \mathrm{~mm}$ & $5.05 \mathrm{~mm}$ & $5.05 \mathrm{~mm}$ & $5.05 \mathrm{~mm}$ \\
\hline row 8 & XXXXXXX & $5.46 \mathrm{~mm}$ & $5.46 \mathrm{~mm}$ & $5.87 \mathrm{~mm}$ & $5.87 \mathrm{~mm}$ & XXXXXXX \\
\hline row 9 & XXXXXXX & XXXXXXX & $5.25 \mathrm{~mm}$ & $5.25 \mathrm{~mm}$ & XXXXXXX & XXXXXXX \\
\hline
\end{tabular}

Average Thickness for A-138-i $=6.10 \mathrm{~mm}$ 
Table 9

A-138-2, Right Side

\begin{tabular}{|l|l|l|l|l|l|l|l|}
\hline & col. 5 & col. 6 & col. 7 & col. 8 & col. 9 & col. 10 & Col. 11 \\
\hline row 4 & XXXXXX & XXXXXX & $11.05 \mathrm{~mm}$ & $11.05 \mathrm{~mm}$ & $11.05 \mathrm{~mm}$ & XXXXXX & XXXXXX \\
\hline row 5 & XXXXXX & $11.12 \mathrm{~mm}$ & $11.12 \mathrm{~mm}$ & $11.12 \mathrm{~mm}$ & $10.47 \mathrm{~mm}$ & $10.47 \mathrm{~mm}$ & XXXXXX \\
\hline row 6 & $10.89 \mathrm{~mm}$ & $10.89 \mathrm{~mm}$ & $10.89 \mathrm{~mm}$ & $11.06 \mathrm{~mm}$ & $11.06 \mathrm{~mm}$ & $10.81 \mathrm{~mm}$ & $10.81 \mathrm{~mm}$ \\
\hline row 7 & $11.00 \mathrm{~mm}$ & $11.00 \mathrm{~mm}$ & $11.00 \mathrm{~mm}$ & $11.30 \mathrm{~mm}$ & $11.30 \mathrm{~mm}$ & $11.20 \mathrm{~mm}$ & $11.20 \mathrm{~mm}$ \\
\hline row 8 & XXXXXX & $11.04 \mathrm{~mm}$ & $11.04 \mathrm{~mm}$ & $11.04 \mathrm{~mm}$ & $10.95 \mathrm{~mm}$ & $10.95 \mathrm{~mm}$ & XXXXXX \\
\hline row 9 & XXXXXX & XXXXXX & $11.29 \mathrm{~mm}$ & $11.29 \mathrm{~mm}$ & $11.29 \mathrm{~mm}$ & XXXXXX & XXXXXX \\
\hline
\end{tabular}

Table 10

A-138-2, Right Side

\begin{tabular}{|l|l|l|l|l|l|l|}
\hline & col. 4 & col. 5 & Col.6 & col. 7 & col. 8 & Col.9 \\
\hline row 10 & XXXXXXX & XXXXXXX & $11.06 \mathrm{~mm}$ & $11.06 \mathrm{~mm}$ & XXXXXXX & XXXXXXX \\
\hline row 11 & XXXXXXX & $11.00 \mathrm{~mm}$ & $11.00 \mathrm{~mm}$ & $11.15 \mathrm{~mm}$ & $11.15 \mathrm{~mm}$ & XXXXXXX \\
\hline row 12 & $11.16 \mathrm{~mm}$ & $11.16 \mathrm{~mm}$ & $11.16 \mathrm{~mm}$ & $11.49 \mathrm{~mm}$ & $11.49 \mathrm{~mm}$ & $11.49 \mathrm{~mm}$ \\
\hline row 13 & $11.49 \mathrm{~mm}$ & $11.49 \mathrm{~mm}$ & $11.49 \mathrm{~mm}$ & $11.38 \mathrm{~mm}$ & $11.38 \mathrm{~mm}$ & $11.38 \mathrm{~mm}$ \\
\hline row 14 & $11.39 \mathrm{~mm}$ & $11.39 \mathrm{~mm}$ & $11.39 \mathrm{~mm}$ & $11.33 \mathrm{~mm}$ & $11.33 \mathrm{~mm}$ & $11.33 \mathrm{~mm}$ \\
\hline row 15 & XXXXXXX & $11.38 \mathrm{~mm}$ & $11.38 \mathrm{~mm}$ & $11.34 \mathrm{~mm}$ & $11.34 \mathrm{~mm}$ & XXXXXXX \\
\hline row 16 & XXXXXXX & XXXXXXX & $11.32 \mathrm{~mm}$ & $11.32 \mathrm{~mm}$ & XXXXXXX & XXXXXXX \\
\hline
\end{tabular}


Table 13

A-138-3, Right Side

\begin{tabular}{|l|l|l|l|l|l|l|l|}
\hline & col. 5 & col. 6 & col. 7 & col. 8 & col. 9 & col. 10 & Col. 11 \\
\hline row 4 & XXXXXX & XXXXXX & $16.38 \mathrm{~mm}$ & $16.38 \mathrm{~mm}$ & $16.38 \mathrm{~mm}$ & XXXXXX & XXXXXX \\
\hline row 5 & XXXXXX & $17.12 \mathrm{~mm}$ & $17.12 \mathrm{~mm}$ & $17.12 \mathrm{~mm}$ & $16.34 \mathrm{~mm}$ & $16.34 \mathrm{~mm}$ & XXXXXX \\
\hline row 6 & $17.04 \mathrm{~mm}$ & $17.04 \mathrm{~mm}$ & $16.75 \mathrm{~mm}$ & $16.75 \mathrm{~mm}$ & $16.38 \mathrm{~mm}$ & $16.38 \mathrm{~mm}$ & $16.38 \mathrm{~mm}$ \\
\hline row 7 & $17.01 \mathrm{~mm}$ & $17.02 \mathrm{~mm}$ & $17.02 \mathrm{~mm}$ & $16.99 \mathrm{~mm}$ & $16.99 \mathrm{~mm}$ & $17.04 \mathrm{~mm}$ & $17.04 \mathrm{~mm}$ \\
\hline row 8 & $\mathrm{XXXXXX}$ & $17.25 \mathrm{~mm}$ & $17.25 \mathrm{~mm}$ & $17.25 \mathrm{~mm}$ & $17.25 \mathrm{~mm}$ & $17.25 \mathrm{~mm}$ & $X X X X X X$ \\
\hline row 9 & $\mathrm{XXXXXX}$ & $\mathrm{XXXXXX}$ & $17.18 \mathrm{~mm}$ & $17.18 \mathrm{~mm}$ & $17.18 \mathrm{~mm}$ & $\mathrm{XXXXXX}$ & $\mathrm{XXXXXX}$ \\
\hline
\end{tabular}

Table 14

A-138-3, Right Side

\begin{tabular}{|l|l|l|l|l|l|l|}
\hline & col. 4 & col. 5 & Col. 6 & col. 7 & col. 8 & Col.9 \\
\hline row 10 & XXXXXXX & XXXXXXX & $17.15 \mathrm{~mm}$ & $17.15 \mathrm{~mm}$ & XXXXXXX & XXXXXXX \\
\hline row 11 & XXXXXXX & $17.12 \mathrm{~mm}$ & $17.12 \mathrm{~mm}$ & $17.02 \mathrm{~mm}$ & $17.02 \mathrm{~mm}$ & XXXXXXX \\
\hline row 12 & $17.19 \mathrm{~mm}$ & $17.19 \mathrm{~mm}$ & $17.19 \mathrm{~mm}$ & $17.19 \mathrm{~mm}$ & $17.19 \mathrm{~mm}$ & $17.19 \mathrm{~mm}$ \\
\hline row 13 & $17.81 \mathrm{~mm}$ & $17.81 \mathrm{~mm}$ & $17.81 \mathrm{~mm}$ & $17.81 \mathrm{~mm}$ & $17.81 \mathrm{~mm}$ & $17.81 \mathrm{~mm}$ \\
\hline row 14 & $18.15 \mathrm{~mm}$ & $18.15 \mathrm{~mm}$ & $18.15 \mathrm{~mm}$ & $18.15 \mathrm{~mm}$ & $18.15 \mathrm{~mm}$ & $18.15 \mathrm{~mm}$ \\
\hline row 15 & XXXXXXX & $18.27 \mathrm{~mm}$ & $18.27 \mathrm{~mm}$ & $18.27 \mathrm{~mm}$ & $18.27 \mathrm{~mm}$ & $X X X X X X X$ \\
\hline row 16 & XXXXXXX & $X X X X X X X$ & $18.06 \mathrm{~mm}$ & $18.06 \mathrm{~mm}$ & XXXXXXX & XXXXXXX \\
\hline
\end{tabular}


Table 15

A-138-3, Left Side

\begin{tabular}{|l|l|l|l|l|l|l|}
\hline & col. 19 & col. 20 & Col. 21 & col. 22 & col. 23 & Col. 24 \\
\hline row 4 & XXXXXXX & XXXXXXX & $16.49 \mathrm{~mm}$ & $16.49 \mathrm{~mm}$ & XXXXXXX & XXXXXXX \\
\hline row 5 & XXXXXXX & $16.29 \mathrm{~mm}$ & $16.29 \mathrm{~mm}$ & $16.57 \mathrm{~mm}$ & $16.57 \mathrm{~mm}$ & XXXXXXX \\
\hline row 6 & $16.11 \mathrm{~mm}$ & $16.11 \mathrm{~mm}$ & $16.11 \mathrm{~mm}$ & $16.25 \mathrm{~mm}$ & $16.25 \mathrm{~mm}$ & $16.25 \mathrm{~mm}$ \\
\hline row 7 & $16.15 \mathrm{~mm}$ & $16.15 \mathrm{~mm}$ & $16.15 \mathrm{~mm}$ & $16.33 \mathrm{~mm}$ & $16.33 \mathrm{~mm}$ & $16.33 \mathrm{~mm}$ \\
\hline row 8 & XXXXXXX & $16.63 \mathrm{~mm}$ & $16.63 \mathrm{~mm}$ & $16.63 \mathrm{~mm}$ & $16.54 \mathrm{~mm}$ & XXXXXXX \\
\hline row 9 & XXXXXXX & XXXXXXX & $16.14 \mathrm{~mm}$ & $16.14 \mathrm{~mm}$ & XXXXXXX & XXXXXXX \\
\hline
\end{tabular}

Average Thickness for A-138-3 $=17.02 \mathrm{~mm}$ 
Table 17

A-138-4, Right Side

\begin{tabular}{|l|l|l|l|l|l|l|l|}
\hline & col. 5 & col. 6 & col. 7 & col. 8 & col. 9 & col. 10 & Col. 11 \\
\hline row 4 & XXXXXX & XXXXXX & $21.6 \mathrm{~mm}$ & $21.6 \mathrm{~mm}$ & $21.6 \mathrm{~mm}$ & XXXXXX & XXXXXX \\
\hline row 5 & XXXXXX & $21.82 \mathrm{~mm}$ & $21.82 \mathrm{~mm}$ & $21.26 \mathrm{~mm}$ & $21.26 \mathrm{~mm}$ & $21.84 \mathrm{~mm}$ & XXXXXX \\
\hline row 6 & $21.88 \mathrm{~mm}$ & $21.88 \mathrm{~mm}$ & $21.95 \mathrm{~mm}$ & $21.95 \mathrm{~mm}$ & $21.88 \mathrm{~mm}$ & $21.88 \mathrm{~mm}$ & $21.88 \mathrm{~mm}$ \\
\hline row 7 & $21.87 \mathrm{~mm}$ & $21.87 \mathrm{~mm}$ & $21.87 \mathrm{~mm}$ & $21.87 \mathrm{~mm}$ & $21.87 \mathrm{~mm}$ & $22.96 \mathrm{~mm}$ & $22.96 \mathrm{~mm}$ \\
\hline row 8 & XXXXXX & $22.28 \mathrm{~mm}$ & $22.28 \mathrm{~mm}$ & $22.74 \mathrm{~mm}$ & $22.74 \mathrm{~mm}$ & $22.74 \mathrm{~mm}$ & XXXXXX \\
\hline row 9 & XXXXXX & XXXXXX & $21.96 \mathrm{~mm}$ & $21.96 \mathrm{~mm}$ & $21.96 \mathrm{~mm}$ & XXXXXX & XXXXXX \\
\hline
\end{tabular}

Table 18

A-138-4, Right Side

\begin{tabular}{|l|l|l|l|l|l|l|}
\hline & col. 4 & col. 5 & Col. 6 & col. 7 & col. 8 & col. 9 \\
\hline row 10 & XXXXXXX & XXXXXXX & $21.88 \mathrm{~mm}$ & $21.88 \mathrm{~mm}$ & XXXXXXX & XXXXXXX \\
\hline row 11 & XXXXXXX & $22.15 \mathrm{~mm}$ & $22.15 \mathrm{~mm}$ & $21.56 \mathrm{~mm}$ & $21.56 \mathrm{~mm}$ & XXXXXXX \\
\hline row 12 & $22.24 \mathrm{~mm}$ & $22.24 \mathrm{~mm}$ & $22.24 \mathrm{~mm}$ & $22.36 \mathrm{~mm}$ & $22.36 \mathrm{~mm}$ & $22.36 \mathrm{~mm}$ \\
\hline row 13 & $22.84 \mathrm{~mm}$ & $22.84 \mathrm{~mm}$ & $22.84 \mathrm{~mm}$ & $22.58 \mathrm{~mm}$ & $22.58 \mathrm{~mm}$ & $22.58 \mathrm{~mm}$ \\
\hline row 14 & $22.73 \mathrm{~mm}$ & $22.73 \mathrm{~mm}$ & $22.73 \mathrm{~mm}$ & $23.00 \mathrm{~mm}$ & $23.00 \mathrm{~mm}$ & $23.00 \mathrm{~mm}$ \\
\hline row 15 & XXXXXXX & $22.78 \mathrm{~mm}$ & $22.78 \mathrm{~mm}$ & $22.92 \mathrm{~mm}$ & $22.92 \mathrm{~mm}$ & XXXXXXX \\
\hline row 16 & XXXXXXX & XXXXXXX & $22.92 \mathrm{~mm}$ & $22.92 \mathrm{~mm}$ & XXXXXXX & XXXXXXX \\
\hline
\end{tabular}


Table 19

A-138-4, Left Side

\begin{tabular}{|l|l|l|l|l|l|l|}
\hline & col. 19 & col. 20 & Col. 21 & col. 22 & col. 23 & col. 24 \\
\hline row 4 & XXXXXXX & XXXXXXX & $21.85 \mathrm{~mm}$ & $21.85 \mathrm{~mm}$ & XXXXXXX & XXXXXXX \\
\hline row 5 & XXXXXXX & $22.26 \mathrm{~mm}$ & $22.26 \mathrm{~mm}$ & $22.27 \mathrm{~mm}$ & $22.27 \mathrm{~mm}$ & XXXXXXX \\
\hline row 6 & $21.48 \mathrm{~mm}$ & $21.48 \mathrm{~mm}$ & $21.48 \mathrm{~mm}$ & $21.47 \mathrm{~mm}$ & $21.47 \mathrm{~mm}$ & $21.47 \mathrm{~mm}$ \\
\hline row 7 & $21.61 \mathrm{~mm}$ & $21.61 \mathrm{~mm}$ & $21.61 \mathrm{~mm}$ & $21.20 \mathrm{~mm}$ & $21.20 \mathrm{~mm}$ & $21.20 \mathrm{~mm}$ \\
\hline row 8 & XXXXXXX & $21.54 \mathrm{~mm}$ & $21.54 \mathrm{~mm}$ & $21.27 \mathrm{~mm}$ & $21.27 \mathrm{~mm}$ & XXXXXXX \\
\hline row 9 & XXXXXXX & XXXXXXX & $21.36 \mathrm{~mm}$ & $21.36 \mathrm{~mm}$ & XXXXXXX & XXXXXXX \\
\hline
\end{tabular}

Average Thickness for A-138-4 $=22.07 \mathrm{~mm}$ 